
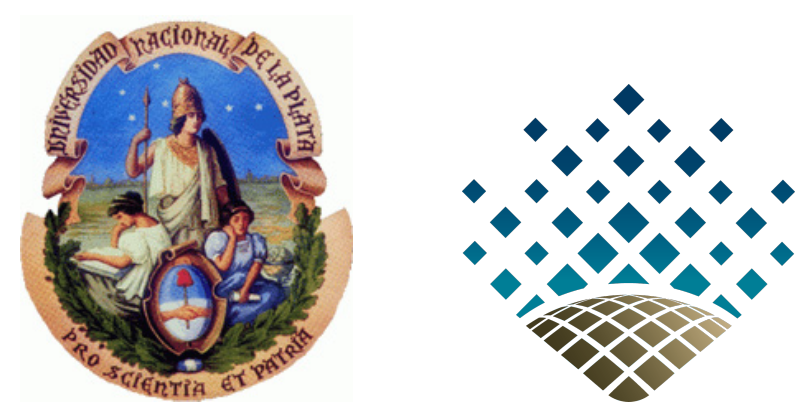

\title{
ESTUDIO EMPÍRICO DE LA DIFUSIÓN CAÓTICA EN SISTEMAS
}

Trabajo de tesis elaborado por el Lic.

LUCIANO ARIEL DARRIBA

Bajo la dirección del

Dr. Pablo Cincotta

y la

Dra. Claudia Giordano

Trabajo presentado para optar por el grado de DOCTOR EN ASTRONOMÍA 



\section{AGRADECIMIENTOS}

A diferencia de lo que uno creería, esta parte del manuscrito de la tesis doctoral es una de las más complicadas de redactar. Principalmente, porque uno no quiere olvidarse de ninguna de las personas que formaron parte de este camino, por más pequeña que haya sido su contribución. Pero también, y creo que es un sentimiento fundamental, es que uno quiere dedicarle a cada una de las personas mencionadas un agradecimiento acorde al impacto que ésta causó en el doctorando. He llegado a la conclusión, muy a pesar de mi obsesión por cuantificarlo todo, de que es muy difícil asignarle un "peso" a este impacto. Por ello voy a tratar de ser lo más justo posible.

Primero, quisiera agradecer a mis directores, el Dr. Pablo Cincotta y la Dra. Claudia Giordano, quienes me orientaron a lo largo del doctorado, y le han dedicado una gran cantidad de tiempo a mi aprendizaje, como también a la supervisión de la escritura de esta tesis. Particularmente gracias Clau, por ser mi suerte de madrina académica, acompañándome a lo largo de toda mi formación, y también por permitirme reunirnos fuera del horario de trabajo en tu casa, dedicándome parte de tu tiempo personal.

A mis jurados, Daniel Carpintero, Cristian Beaugé y Carlos Briozzo, quienes han leído minuciosamente este texto y lo han pulido para que llegue a ser lo que es hoy. Sobre todo agradecerle a Daniel quien, sin dejar pasar ni una sola regla ortográfica de la Real Academia Española de Letras, me ha señalado cada acento, coma o punto, como también hasta el más irrelevante símbolo mátematico presente, para que esta tesis quede impecable. Gracias.

Como se verá más adelante en este escrito, esta tesis consta de dos grandes partes, en cada una de las cuales tuve el placer de trabajar no solamente con excelentísimos investigadores, sino también maravillosas personas. La primera de estas partes fue fruto de un trabajo en colaboración con el Dr. Nicolás Maffione quien, al encontrarse en una etapa más avanzada de su doctorado, me ha ayudado a entender una gran cantidad de conceptos, sobre todo en regularidad y caos. También ha sido muy importante en otras instancias de mi doctorado, las cuales mencionaré llegado el momento. Por lo pronto, gracias Nico por ser el autor intelectual de nuestro (espero que primero de muchos) proyecto, el LP-VIcode, y permitirme ser el autor material.

La segunda parte de esta tesis fue realizada en una estadía de seis meses en la ciudad de Patras, Grecia, gracias al otorgamiento de una beca del proyecto 
Eurotango 2, en el marco del programa Erasmus Mundus, el cual vincula universidades de todo el mundo con universidades de Europa. Este trabajo fue llevado a cabo bajo la supervisión del profesor Tassos Bountis, al cual le agradezco sus contribuciones y las discusiones que hemos tenido, enriqueciéndome enormemente. Durante esta estancia, he conocido y trabajado estrechamente con el profesor Christos Efthymiopoulos, quien me introdujo al mundo de las formas normales, y ha invertido una cantidad enorme de su tiempo en explicarme (con la dedicación de un maestro de escuela primaria) cada uno de los conceptos introducidos. No solamente fue esencial desde un punto de vista académico, sino que también tuvo la humanidad de insistirme en que me tomara mis descansos y que paseara, haciendo las veces de asesor turístico.

Por todo lo expresado aquí, es evidente que no tengo palabras suficientes para agradecer la influencia positiva que ha tenido (y sigue teniendo) en mi carrera, tanto durante como después del doctorado. Lo único que me resta

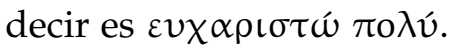

Si bien no ha formado parte directa de ninguna de estas dos instancias, también quiero agradecer al Dr. Martín Mestre, quien ha estado constantemente contribuyendo y auxiliándome cada vez que lo necesité, que no fueron pocas. Él siempre tuvo tiempo para sentarse al lado mío y explicarme con detalle la respuesta a cada una de mis preguntas, por más sencilla que fuera.

También estuvo allí el Dr. Felipe Wachlin, de quien aproveché tanto su pasado en el ámbito de la Dinámica como de sus conocimientos programacionales, cada vez que yo asomaba mi nariz por encima del monitor y lo interrumpía con un "Che, Felipe ...". Gracias Felipe.

Pero el doctorado no es solamente una instancia académica, también tiene una componente social importante. Y acá entran de nuevo a la cancha Nico, Martín y Felipe, a los que se les incorporan Alejandra y, posteriormente, Julieta. Con ellos (y a partir de acá voy a usar un lenguaje menos formal y más cotidiano) he compartido un montón de almuerzos, quedándonos riendo de cualquier pavada, haciéndome olvidar de cualquier frustración que tenía porque no me compilaban los programas. De ellos aprendí muchos valores. De Nico aprendí cómo uno puede seguir siendo un tipo honesto y correcto, por más que lo que te rodea te tire para abajo y no te deje enderezar. De Felipe aprendí cómo ser una persona objetiva, más allá de que uno ya tenga una opinión sobre el asunto, cualquiera fuese el tópico de la conversación. De Ale aprendí que si uno se focaliza con fuerza en lo que hace, no te para nadie. De Martín, con su imaginación colorida y su ocurrencia en los chistes, aprendí que no se puede ser una mente brillante sin colorearla con una pincelada de "locura". Y Juli, aunque la conozco desde hace menos tiempo que al resto, me bastó para aprender que, incluso cuando a uno no le salen las cosas, siempre puede encontrar 
una razón para sonreir. A ustedes, reunidos a almorzar alrededor de la mesa para PC, gracias.

En todo este camino también me he cruzado con muchísima gente con la que estoy muy agradecido, la cual no puedo listar en este apartado, sino duplicaría la cantidad de páginas de esta tesis. En ellos incluyo a los compañeros con los que ingresé a la facultad, los que conocí a lo largo de cursadas, los que fui conociendo a lo largo del doctorado, que me han ayudado a llevar esta carrera con mayor facilidad.

Aunque uno suele olvidarse de ellos, también quiero agradecerle a los alumnos que he tenido a lo largo de estos años, porque ellos son los que vienen con las dudas y preguntas, y han logrado despertar más de una vez en mí esa misma curiosidad que me hizo elegir una carrera científica. Ya que, si uno no tiene el bichito de la curiosidad por aprender, la vida sería muy plana y aburrida.

A los "Planetarios", Gonza, Octavio, Paula, Macarena, Agustín, Irina, Carolina, Patricio, Ximena y el Edu, con quienes compartí un taller de ciencias planetarias en el final de mi doctorado y, honestamente, me hicieron sentir muy bienvenido a mi nuevo grupo. Gracias chicos.

Para otro de los "Planetarios", Pablo Santamaría, tengo un especial agradecimiento. No solo por hacerme sentir bien en ese grupo, sino porque con él hemos charlado una innumerable cantidad de veces, introduciéndome a Fortran 90 (y respondiendo todas las preguntas que pudieran surgir en el momento de aprendizaje), resolviendo todas mis dudas sobre Linux y el mundo de las computadoras. Gracias Pablo.

A los Magios, con quienes hemos pasado una gran cantidad de reuniones comiendo y jugando al truco. Lo que nos hemos reído imaginándonos que nuestras esposas pensarían que estábamos de joda, y en realidad nuestro divertimento más "zarpado"era ver Los Simpsons o La Pantera Rosa. Muchas gracias.

$Y$ para enfrentarse a la bestia que representa una carrera universitaria, uno no puede lograrlo sin la ayuda de sus escuderos. $Y$ en mi vida mis escuderos fueron mi familia. Ellos me apoyaron desde que era muy chico, y en ese entonces ya me apasionaba el Universo y su inmensidad. Ellos entendieron que la astronomía era mi futuro, y nunca permitieron que me apartara del camino. A mis viejos, los cuales literalmente se rompieron el lomo trabajando para que yo pudiera estudiar y llegar a ser lo que soy, les estoy eternamente agradecido. También a mis hermanos Leandro, Germán y Carli, y a mi tía Ana, gracias por estar siempre a mi lado. A mi abuela quien, a su manera italiana, me consentía con sus comidas por ser el nieto varón más chico. A mi abuelo, que ya no está en este planeta (quién sabe qué sistemas esté recorriendo ahora), que cada 
vez que quería medirse la presión venía a buscarme, buscando a su "dottore". Ahora sí nono, ahora sí soy "dottore". A todos ustedes les dedico este trabajo.

Y lo mejor me lo guardé para el final. Euge. Como lo resumí en la frase anterior, Euge es simplemente lo mejor. Lo mejor que me pasó en la vida. Es mi amor, mi compañera, mi bastón. Siempre que necesité de alguien estuvo ahí, aunque eso haya significado que tuviera que posponer (o incluso renunciar) sus cosas para dedicarse a mí. Este doctorado no podría haberlo llevado a cabo sin su apoyo, levantándome el ánimo ante todas las adversidades que pasé en estos cinco años. Adversidades que me llevaron incluso a pensar que quizá no servía para la investigación. Allí también me alentó a seguir adelante, y no dejarme vencer. Este trabajo va dedicado a vos. Eternamente gracias !!!!!!! 


\section{ORIGINALIDAD}

Los frutos de este trabajo de tesis fueron la publicación de dos trabajos regulares y un trabajo de revsión en revistas internacionales con referato, las cuales se encuentran detalladas a continuación:

\section{Trabajos regulares:}

- Título: A comparison of different indicators of chaos based on the deviation vectors. Application to symplectic mappings.

Autores: Maffione, N.P., Darriba, L.A., Cincotta, P.M., Giordano, C.M.

Revista: Celest. Mech. Dyn. Astron., 111, 285-307

Año: 2011

- Título: Chaos detection tools: application to a self-consistent triaxial model

Autores: Maffione, N.P., Darriba, L.A., Cincotta, P.M., Giordano, C.M.

Revista: Mon. Not. R. Astron. Soc., 429, 2700-2717

Año: 2013

\section{Trabajo de revisión:}

- Título: Comparative study of variational chaos indicators and ODEs' numerical integrators

Autores: Darriba, L.A., Maffione, N.P., Cincotta, P.M., Giordano, C.M.

Revista: Int. J. Bifurcation and Chaos., 22, 1230033(33)

Año: 2012

Además de las publicaciones regulares y el trabajo de revisión, la creación del código LP-VIcode dio lugar a un capítulo de un libro en el marco de una escuela internacional:

- Título del capítulo: Chaos detection tools: The LP-VIcode and its applications

Autores: Darriba, L.A., Maffione, N.P., Cincotta, P.M., Giordano, C.M.

Publicación: En: P.M. Cincotta, C.M. Giordano \& C. Efthymiopoulos eds.: 3rd La Plata International School on Astronomy and Geophysics "Chaos, 
Diffusion and Non-integrability in Hamiltonian Systems. Application to Astronomy ", 345-366

Año: 2012

viii 
Cuando queremos estudiar la dinámica de un sistema, por ejemplo una galaxia o un sistema planetario, es importante primero conocer en qué regiones del sistema una órbita tiene un comportamiento regular y en cuáles un comportamiento caótico. Las herramientas que utilizaremos para abordar esta cuestión son los llamados indicadores de caos.

Existen en la literatura una gran cantidad de estos indicadores, de los cuales en este trabajo utilizaremos aquéllos basados en la evolución de la solución de las ecuaciones variacionales. Algunos ejemplos de este tipo de indicadores son el Máximo Exponente de Lyapunov (ILCE), el Indicador Rápido de Lyapunov (FLI) y su variante que considera solo la componente ortogonal (OFLI), el Factor de Crecimiento Exponencial Medio de Órbitas Cercanas (MEGNO), el Índice Menor de Alineamiento (SALI), entre otros.

En el Capítulo 2 revisaremos las principales características de una variedad de este tipo de indicadores. Luego, en el Capítulo 3 presentaremos un código, escrito en FORTRAN, que integra de una forma eficiente todos los indicadores descriptos en el Capítulo 2. Hemos desarrollado dos versiones de este programa, una para mapas simplécticos y otra para flujos hamiltonianos. La primera será empleada en la segunda parte de este trabajo, y ambas versiones fueron utilizadas en [55].

La segunda parte de este trabajo está dedicada al estudio, dentro de la región caótica, de la difusión, esto es, determinar si existe una variación secular de las integrales no perturbadas del sistema.

Para valores perturbativos muy pequeños se encontraron escenarios en los que la difusión no era detectable a causa de las oscilaciones introducidas por los efectos de deformación del conjunto de variables utilizadas, por lo que recurrimos al uso de las formas normales. Dado que no existía hasta el momento una implementación de esta técnica para el caso de mapas, en este trabajo se creó, por primera vez en la literatura, dicha implementación. Esta herramienta es una sucesión de transformaciones canónicas que permite describir, de una forma más clara, la dinámica del sistema, eliminando justamente los efectos de deformación. En el Capítulo 4 presentaremos, de una manera detallada, el mecanismo para la construcción de las formas normales para un mapa simpléctico 4D cuasi-torsional general. En el Capítulo 5 mostraremos cómo aplicar dicho mecanismo a dos mapas estándar acoplados. Dado que las formas normales se construyen mediante series de Fourier, presentaremos los resultados de la medición de los tiempos de CPU empleados para distintos órdenes de este 
desarrollo. También presentaremos una estimación empírica del orden óptimo para el cual construir la forma normal, para dos escenarios distintos. Finalmente, en el Capítulo 6 llevaremos a cabo el estudio de la difusión, que es el objetivo central de este trabajo. Este estudio lo realizaremos a través de la medición de la desviación cuadrática media de la acción en la dirección de la resonancia con respecto a su valor inicial. Estudiaremos un ensamble de $10^{3}$ partículas considerando varios escenarios distintos mediante la variación del parámetro de acoplamiento del mapa. 


\section{ÍNDICE GENERAL}

1 PRÓLOGO

i DETECCIÓN DEL CAOS

2 INDICADORES DE CAOS (ICS) 9

2.1 Conceptos básicos . . . . . . . . . . . . . . 10

2.1.1 Sistema dinámico . . . . . . . . . . . . . . 10

2.1.2 Sistemas dinámicos continuos - sistemas hamiltonianos . 10

2.1.3 Ecuaciones variacionales . . . . . . . . . . . . . II

2.1.4 Sistemas dinámicos discretos - Mapas . . . . . . . . . . 12

2.1.5 Mapa tangente . . . . . . . . . . . . . . . . . . . . 13

2.1.6 Definición de caos . . . . . . . . . . . . . . 13

2.2 Definiciones de los indicadores de caos . . . . . . . . . . 14

2.2.1 El Indicador de Lyapunov $(L I) \ldots \ldots \ldots$

2.2.2 El Indicador Relativo de Lyapunov $(R L I) \ldots \ldots . . . . . .16$

2.2.3 Los Espectros Dinámicos de los Números de Dilatación Local (SSNs) y la Distancia Espectral (DS) . . . . . . . 17

2.2.4 El Factor de Crecimiento Exponencial Medio entre Órbitas Cercanas $(M E G N O)$. . . . . . . . . . . 18

2.2.5 El Índice Menor de Alineamiento $(S A L I)$ y el Índice de Alineamiento Generalizado $(G A L I) \quad \ldots \ldots 20$

2.2.6 El Indicador Rápido de Lyapunov $(F L I)$ y la componente Ortogonal del Indicador Rápido de Lyapunov (OFLI) . . . 22

2.2.7 La componente Ortogonal del Indicador Rápido de Lyapunov de segundo orden $\left(O F L I_{\mathrm{TT}}^{2}\right) \ldots \ldots . \ldots 23$

2.2.8 El Exponente Medio de Ley de Potencias (APLE) . . . . 24

3 UN Código Para CALCUlar ics: EL LP-VICOde 25

3.1 Características generales del LP-VIcode . . . . . . . . . . . . . 25

3.2 El programa principal . . . . . . . . . . . . . . . . . . . . 26

3.3 Entrada de datos . . . . . . . . . . . . . . . . 27

3.4 El Integrador . . . . . . . . . . . . . . . . . . . 28

3.5 Cálculo de $\operatorname{los}$ ICs . . . . . . . . . . . . . . . . . 28

3.6 El ordenamiento de los ICs en unidades . . . . . . . . . . . . . 29

3.6.1 Unidad 1 . . . . . . . . . . . . . . . . . . . . 29

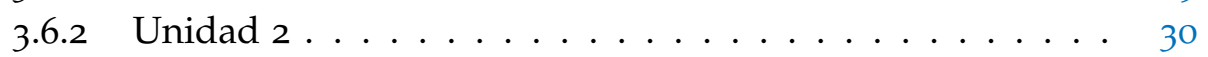

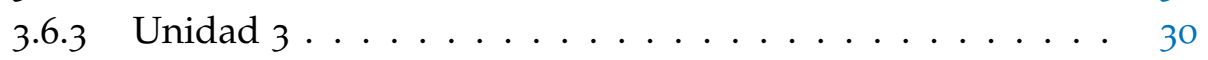

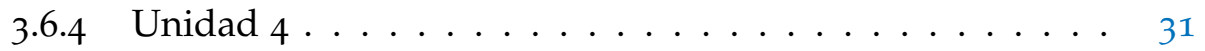

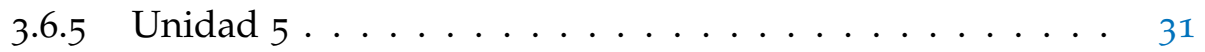




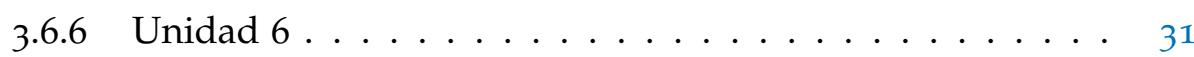

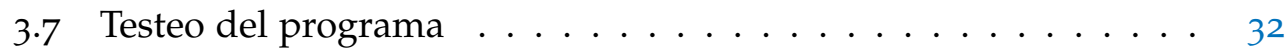

$3 \cdot 7.1$ Tiempos de CPU . . . . . . . . . . . . . 33

3.7 .2 Aplicación a mapas simplécticos . . . . . . . . . . 36

$3.7 \cdot 3$ Aplicación a flujos hamiltonianos . . . . . . . . . . . . 39

3.8 Discusión . . . . . . . . . . . . . . . . . . . 49

ii CUANTIFICACión DE La difusión 51

4 estudio de la difusión mediante el Cálculo de formas NORMALES 53

4.1 Definiciones y conceptos básicos ................. 55

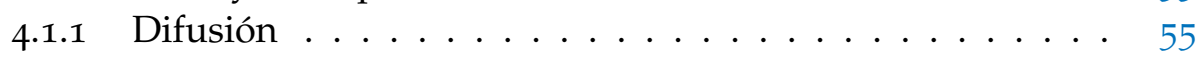

4.1.2 El mapa de torsión . . . . . . . . . . . . . . . . 57

4.2 El Mapa Estándar Racional Acoplado y Desplazado (CRSSM) . . 59

4.2.1 Selección de las cantidades a calcular . . . . . . . . . 6 61

4.3 Estimación de la difusión para distintas magnitudes de la perturbación . . . . . . . . . . . . . . . . 63

4.4 Método para el cálculo de la forma normal en mapas . . . . . . . 67

4.4.1 Selección y preparación del mapa . . . . . . . . . . . . . 67

4.4.2 El parámetro de contabilidad . . . . . . . . . 70

4.4 .3 Obtención de la ecuación de conjugación . . . . . . . . 72

4.4.4 Construcción y resolución de la ecuación homológica . . . 75

4.4 .5 Comprobación de simplecticidad . . . . . . . . . . 80

4.4 .6 Transformación inversa . . . . . . . . . . . 82

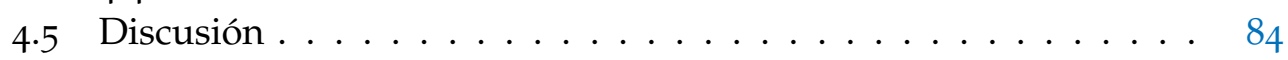

5 APLiCACión A UN MAPA SIMPléctico 4D 87

5.1 Aplicación del cálculo de formas normales al CRSSM . . . . . . . 87

5.2 Resultados ......................... 91

5.2.1 Tiempos de cómputo . . . . . . . . . . . . . 92

5.2.2 Precisión de la transformación y estimación empírica del

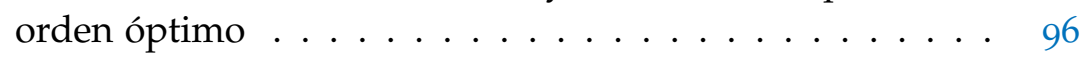

5.3 Discusión ........................... 106

6 CUANTIFICACIÓN DE LA Difusión 107

6.1 Preparación del entorno para el estudio de la difusión . . . . . . 107

6.1.1 Condiciones iniciales . . . . . . . . . . . . . . 108

6.2 Gráficos comparativos pre y post-transformación . . . . . . . . 110

6.3 Caracterización de la difusión en una resonancia simple . . . . 116

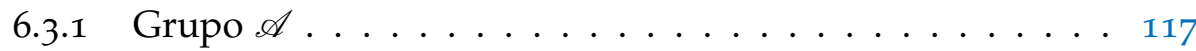

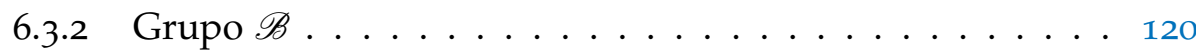

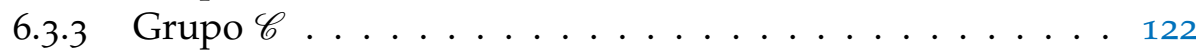

6.3.4 Estimación del coeficiente de difusión $\mathcal{D} \ldots \ldots \ldots$. . . . . 124 
6.4 Caracterización de la difusión en las cercanías de un cruce de resonancias . . . . . . . . . . . . . . . . . 129

6.5 Discusión . . . . . . . . . . . . . . . . . . . . . 134

7 CONCLUSIONES 137

BIBLIOGRAFÍA 143

A COMPOSICIÓN ENTRE SERIES DE FOURIER 151

B FUNCIÓN DE INDEXACIÓN 155

C FORMAS NORMALES: El Código 159

C.1 Consideraciones generales . . . . . . . . . . . . . . 160

C.1.1 Series y arreglos . . . . . . . . . . . . . 160

C.2 public_values.f90 . . . . . . . . . . . . . 160

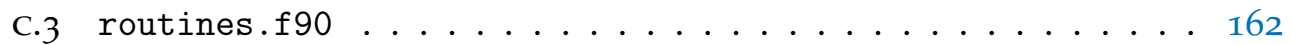

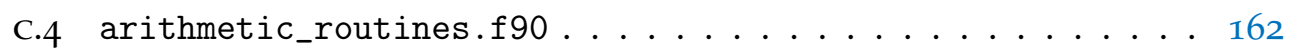

C.5 compose_functions.f90 . . . . . . . . . . . . . 164

c.6 inverse_transformation.f90 . . . . . . . . . . . . 165

C.7 build_mapping.f90 . . . . . . . . . . . . . . 165

c.8 main.f90 . . . . . . . . . . . . . . . . . . . . 165

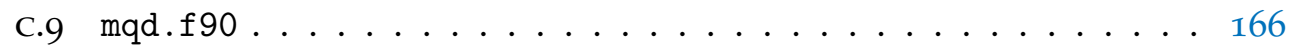





\section{PRÓLOGO}

Existen en la naturaleza una gran cantidad de fenómenos que pueden ser modelados matemáticamente mediante sistemas dinámicos. Es posible encontrar estos fenómenos en áreas como la física, la biología, la química y, en el área de la astronomía, podemos mencionar sistemas como nuestro Sistema Solar, sistemas planetarios extrasolares, cúmulos estelares, galaxias, etc. Dentro de los sistemas dinámicos en la astronomía dinámica, resultan de interés los sistemas hamiltonianos. Las ecuaciones que describen estos sistemas son ecuaciones deterministas, es decir, que no poseen ninguna componente aleatoria. Sin embargo, esta característica no es suficiente para asegurar que, para una dada condición inicial, la trayectoria a un cierto instante de tiempo se comportará de forma absolutamente predecible. La presencia de términos no lineales en las ecuaciones lo vuelven sensible ante la variación de la condición inicial (incluso si ésta es tan pequeña como el error de redondeo originado por el empleo de técnicas numéricas). Este fenómeno es denominado en la actualidad como caos, y es uno de los temas de mayor impacto en el ámbito de la Dinámica No Lineal.

La sensibilidad ante una variación en las condiciones iniciales antes mencionadas hace que dos valores iniciales muy próximos en el espacio de fases, se separen exponencialmente con el tiempo (al menos localmente), dando lugar a trayectorias completamente diferentes entre sí. Por lo tanto, si existe un conjunto de valores iniciales ubicado en una región acotada del espacio, cuando evolucionen en el tiempo cada uno podría, en principio, visitar distintas regiones. Este hecho hace parecer que el caos es aleatorio y, por lo tanto, impredecible. Aun así, distintas regiones de un sistema podrían manifiestan distintos grados de caoticidad ([17], [42], [51] entre otros). Luego, para regiones donde el nivel de caos presente es moderado o bajo, es posible estudiar el comportamiento a largo plazo de la evolución de valores iniciales que se encuentren dentro de dicho sistema.

Para realizar este estudio es necesario contar con algún mecanismo para detectar el caos y cuantificarlo. Para sistemas de 2 grados de libertad, existen métodos gráficos capaces de detectar si una región es caótica o regular. Uno de estos métodos es el estudio de Superficies de Sección, introducido por Henri 
Poincaré (1854-1912) ([68], [72]). Para sistemas de 3 o más grados de libertad, el empleo de métodos gráficos se tornó una tarea muy compleja, por lo que fue necesario el desarrollo de métodos no gráficos, que llamaremos indicadores de caos. Algunos indicadores que se pueden encontrar en la literatura son los basados en el análisis espectral, como la Transformada Modificada de Fourier en las Frecuencias ([77]), o también basados en los Algoritmos Evolutivos ([67]). Un conjunto muy interesante de indicadores son los basados en la evolución de la solución de las ecuaciones variacionales primeras, a los cuales llamaremos indicadores variacionales para diferenciarlos de los otros tipos de indicadores.

Los primeros métodos de esta clase estaban basados en la divergencia exponencial local entre dos órbitas con valores iniciales próximos. Este concepto de divergencia exponencial fue introducido por Aleksandr Lyapunov (1857-1918), quien estableció un criterio de estabilidad para la solución de ecuaciones diferenciales ordinarias (EDOs), mucho antes de que existiera el concepto de caos ([54]). Varios años más tarde, se implementó este criterio para analizar la estabilidad de las EDOs correspondientes a las ecuaciones de movimiento que describen la trayectoria de una dada condición inicial. Uno de los primeros mecanismos para detectar el caos fue a través del cálculo del llamado LCE (del inglés Lyapunov Characteristic Exponent, ver Sección 2.2.1). Este indicador establece que dos condiciones iniciales muy cercanas, dentro de una región caótica, divergen exponencialmente, y el valor de este exponente fue llamado $L C E$ ([64]).

En los últimos años se han desarrollado una gran cantidad de indicadores variacionales. Debido a esto, surgió la idea de poder contar con un programa que, de acuerdo al estudio estadístico que se desea realizar, calcule determinados indicadores. Esto dio origen a la creación del LP-VIcode (del inglés La Plata Variational Indicators code), un código escrito en Fortran 77 que implementa y calcula de forma eficiente algunos de estos indicadores variacionales. Aquellos indicadores que han sido implementados en este código son: el Indicador de Lyapunov (LI, [6]), el Indicador Relativo de Lyapounov (RLI, [73]), el Factor de Crecimiento Exponencial Medio entre Órbitas Cercanas (MEGNO, [16]) y la estimación de su pendiente (SElLCE, [17]), el Indicador Rápido de Lyapunov (FLI, [36]), su variante que calcula la componente ortogonal del FLI (OFLI, [32]) y también la que calcula esta componente utilizando ecuaciones variacionales de segundo orden (OFLI TT, [1]), el Exponente Medio de Ley de Potencias (APLE, [53]), los Espectros de los Números de dilatación local (SSN, [86]) y la Distancia Espectral (DS, [87]), el Índice Menor de Alineamiento (SALI, [78]) y su generalización, el Índice Generalizado de Alineamiento (GALI $2 \mathrm{~N}$, [81]). Este código es capaz de calcular varios indicadores simultáneamente en un tiempo considerablemente menor al empleado en el cálculo de cada uno de los indicadores calculados previamente de manera independiente. 
Se han creado dos versiones de este código: una para sistemas discretos (mapas simplécticos) y otra para sistemas continuos (flujos hamiltonianos). Ambas versiones han sido utilizadas en [55]. Este código ha dado lugar a la publicación de tres artículos en revistas internacionales ([24], [56], [58]), un capítulo de un libro ([23]) y fue una herramienta fundamental para la detección de la estructura dinámica del sistema con el que trabajaremos en la segunda parte de esta tesis.

Volviendo al estudio de la inestabilidad de una dada trayectoria, cabe destacar que, a pesar de que la distancia entre dos órbitas con condiciones iniciales próximas, ubicadas en una región caótica, diverja exponencialmente en el tiempo, no implica que sus trayectorias no se encontrarán acotadas en el espacio. Por ejemplo, en todo sistema astronómico ligado, el espacio de fases es finito, por lo que la distancia máxima entre las dos órbitas previamente mencionadas estará acotada por el tamaño del sistema.

A esta variación secular, cuya magnitud excede un dado valor límite prefijado, se la conoce con el nombre de difusión caótica o, simplemente difusión. Comúnmente se entiende por difusión un fenómeno de dispersión de un conjunto de condiciones iniciales con respecto a un determinado valor medio, el cual se puede cuantificar utilizando herramientas como la varianza $\sigma^{2}$. En la segunda parte de nuestro trabajo, estudiaremos los procesos difusivos que se manifiestan en el espacio de las acciones de un mapa simpléctico. Esta difusión será cuantificada utilizando la desviación cuadrática media.

Para poder detectar y cuantificar la difusión, necesitamos primero conocer la estructura dinámica del espacio de fases, esto es, regiones donde existen toros invariantes que soportan el movimiento cuasiperiódico, resonancias, intersección de resonancias, regiones caóticas extendidas, etc. Utilizaremos el LP-VIcode para identificar las regiones caóticas de nuestro sistema, para luego analizar si las magnitudes que se desean estudiar (representadas a través de acciones) varían en un entorno de tamaño prefijado o si lo exceden.

En la década de 1980 los científicos han utilizado mapas (como el mapa estándar o el mapa de la separatriz) con el fin de estudiar el fenómeno de la difusión en sistemas dinámicos simples, que ocurren en la teoría del movimiento en el Sistema Solar, como por ejemplo difusión a largo plazo de asteroides en resonancia de movimientos medios, dinámica spin-órbita de satélites planetarios, dinámica de los asteroides troyanos de Júpiter, etc (por ejemplo, [30], [52], [83], [84],[88], o ver [76] para un review). Estos mapas han sido incluso aplicados al estudio del fenómeno de difusión a largo plazo en modelos relevantes para la dinámica galáctica ([14], [15]), Los sistemas más simples que podemos encontrar, en los cuales está presente el caos, son sistemas de 2 grados de libertad (g.l.) pero, por lo general, un sistema dinámico que modela la dinámica del Sistema Solar es un sistema de muchos más g.l. que el mencionado, como los 
mostrados en [76]. Por ello, para obtener un mapa con un mayor número de g.l., se debe recurrir al uso de varios mapas acoplados.

Existe una diferencia topológica elemental entre el espacio de fases correspondiente a un sistema hamiltoniano de 2 g.l. con respecto a uno con 3 o más g.1. Esta diferencia refiere a la existencia de ensambles invariantes de la dinámica que se llaman toros KAM (ver [13], [27]). En sistemas de 2 g.l., los toros KAM constituyen obstáculos absolutos para la difusión, mientras que en sistemas con 3 o más g.l. encontramos una nueva posibilidad de que una acción varíe secularmente, que se llama la difusión de Arnold ([13], [27]).

El estudio de la difusión se ha llevado a cabo sobre un mapa simpléctico 4D del tipo cuasi-torsional llamado CRSSM (de sus siglas en inglés Coupled Rational Shifted Standard Map, que puede ser traducido como Mapa Estándar Racional Acoplado y Desplazado), introducido en [17], y descripto más adelante en el Capítulo 4. La elección de este mapa no es aleatoria, sino que ha sido escogido porque matemáticamente puede expresarse, como su nombre lo indica, como dos mapas estándar acoplados. Por otro lado, al ser un mapa 4D, éste corresponde a un sistema hamiltoniano de 3 g.l. De esta manera, este mapa es el más simple que se puede construir, con la suficiente complejidad que permite manifestar la difusión de Arnold.

Como es sabido (ver [42], por ejemplo), para perturbaciones muy pequeñas, la difusión se logra detectar con mucha dificultad, debido a que ésta será tan lenta que las oscilaciones introducidas por el sistema la ocultarán. Esta dificultad se superó mediante la aplicación de la llamada transformación a formas normales. Esta herramienta consiste en una sucesión de transformaciones canónicas que permiten expresar las variables originales en función de las nuevas variables, para construir un nuevo mapa en el cual la dinámica pueda ser descripta de forma más simple y clara.

No existe en la actualidad ninguna implementación de esta técnica para el caso de mapas, sino que solamente se encuentra implementada para flujos hamiltonianos (por ejemplo, [18], [25], [26], [30], [29], [75]). Por este motivo, en este trabajo de tesis realizamos dicha implementación. El algoritmo presentado en el Capítulo 4 es un rediseño aplicado al caso de mapas de una implementación previamente propuesta en [30] para flujos hamiltonianos. Una de las inclusiones más novedosas que incluye esta implementación es la introducción de una técnica algorítmica, llamada book-keeping. Esta técnica se utiliza para ordenar y clasificar los términos de un mapa, expresados como una serie de Fourier, en función del tamaño de sus coeficientes, los cuales decrecen con el orden del desarrollo. Este algoritmo nos permite llevar a cabo la construcción de la transformación a formas normales de forma iterativa.

La implementación de la transformación a formas normales es un algoritmo no trivial que nos permite, por primera vez en la literatura, llevar a cabo expansiones para un mapa genérico $4 \mathrm{D}$ en variables ángulo-acción hasta un orden 
arbitrario. Esto permite llevar las expansiones a órdenes lo suficientemente altos con el fin de detectar (de manera muy clara) y cuantificar la difusión cuando la perturbación introducida en el sistema es muy pequeña. Esta tarea resultaba prácticamente imposible de llevar a cabo en las variables originales del sistema.

Para poder utilizar este método se realizó el estudio detallado de esta técnica, comprendiendo su estructura matemática, para luego poder llevar a cabo su implementación numérica. Esto dio origen a un código, escrito en Fortran 90, que construye la transformación a las formas normales, encuentra la expresión de la nueva forma del mapa (la forma normal) y construye, a partir de esta transformación, la transformación inversa. Esta última nos permite expresar el nuevo conjunto de variables en función del conjunto original para, a partir de la evolución de la órbita en las variables originales, calcular dicha evolución en las nuevas variables. Con la expresión de la transformación inversa calculada, se construyó otro programa que calcula las coordenadas en el nuevo espacio de fases. Este desarrollo es posible escribirlo como una serie de Fourier, por lo que la transformación puede ser calculada hasta un orden arbitrario $\mathcal{O}(\mathrm{N})$. No existe aún en la literatura un mecanismo eficiente para la construcción de las formas normales para mapas simplécticos como el desarrollado en este trabajo. Es por esto que se dedicará el Capítulo 4 a la explicación del mecanismo para la implementación del método a un mapa simpléctico 4D near-twist general y luego, en la primera parte del Capítulo 5, se detallará cómo aplicarlo al caso particular del CRSSM. Una vez implementado el código, se lo probó exhaustivamente, y sus resultados se muestran en la segunda parte del Capítulo 5 . Estos resultados incluyen la medición de los tiempos de CPU empleados por el código, tanto para construir el desarrollo hasta distintos órdenes $\mathcal{O}(\mathrm{N})$, como para la construcción de la nueva serie temporal de las acciones transformadas.

Dado que esta transformación es un desarrollo en serie, a medida que incrementamos el orden del desarrollo, el error disminuye. Pero, al ser una sucesión de transformaciones canónicas, comienzan a aparecer en los denominadores del desarrollo cantidades cercanas a las condiciones de resonancia, conocidas como "pequeños denominadores". Entonces, a medida que aumentamos el orden del desarrollo, el error debido a los pequeños denominadores aumenta. Luego, existe un orden, llamado orden óptimo, el cual nos indica el orden para el que la suma de las contribuciones de los errores debido al orden del desarrollo y a los pequeños denominadores es mínima.

En este trabajo, también se realizó una estimación empírica del orden óptimo para el desarrollo. Finalmente, en el Capítulo 6 se llevó a cabo la caracterización de la difusión en una región del mapa CRSSM para distintos valores del parámetro perturbativo en un intento de estudiar, de manera empírica, cómo se comporta la difusión en una resonancia simple.

Los resultados obtenidos en este trabajo permitieron la redacción de un nuevo artículo, el cual será enviado a la brevedad para su publicación. 
Por todo lo expuesto, este trabajo de tesis estará conformado por dos grandes partes (en el comienzo de cada capítulo se brindará una lista exhaustiva de las correspondientes referencias). En la primera parte, dedicada a la detección del caos, se describirán los indicadores variacionales implementados en el programa LP-VIcode. Luego, se presentará el mencionado programa, detallando sus principales características, junto con la presentación de algunos experimentos que se realizaron con éste. La segunda parte, será dedicada a la caracterización de la difusión. Aquí se detallará la técnica de las transformaciones a formas normales, describiendo paso a paso el mecanismo para la construcción de la mencionada transformación, para un mapa simpléctico genérico 4D. También se mostrará la aplicación de esta técnica al CRSSM. Finalmente, se llevará a cabo la caracterización de la difusión, en búsqueda de regiones que presenten difusión normal. En esas regiones se realizará la cuantificación de la difusión mediante la estimación del coeficiente de difusión $\mathcal{D}$.

Cabe aclarar que el desarrollo de este trabajo se vio fuertemente motivado debido a los escasos resultados concluyentes en la literatura, acerca de la caracterización de la difusión a lo largo de una resonancia simple, en el límite de perturbaciones tendiendo a cero. 
Parte I

DETECCIÓN DEL CAOS 



\section{2}

INDICADORES DE CAOS (ICS)

Cuando queremos estudiar la dinámica de un sistema, como una galaxia, un sistema platenario, entre otros, es de vital importancia conocer las regiones de estabilidad de éste. Esto quiere decir, que es fundamental tener conocimiento acerca de en qué regiones las órbitas que se encuentren allí se comportarán de manera regular, y en qué regiones se comportarán como caóticas. La definición de este último concepto será dada más adelante en este capítulo.

Las herramientas utilizadas para determinar la regularidad o caoticidad de la evolución de una condición inicial inmersa en un potencial son los llamados "indicadores de caos" (ICs en adelante). Existe una gran variedad de técnicas que se utilizan para determinar si una dada trayectoria es regular o caótica. Una de ellas se basa en el análisis y comparación del espectro de frecuencias de la trayectoria ([8], [9], [47], [48], [49], [65], [66]). Otra de las técnicas utilizadas es la basada en la evolución de la solución de las ecuaciones variacionales ([6], [16], [32], [36], [78], [81]). En este trabajo nos focalizaremos en los indicadores pertenecientes a esta última categoría.

Debido a la necesidad de poder contar con herramientas para determinar qué tipo de estructura (en cuanto a las regiones regulares o caóticas) posee un sistema, se desarrolló una herramienta, la cual será presentada en el Capítulo 3 que calcula, de una manera eficiente, una gran cantidad de ICs basados en la evolución de la solución de las ecuaciones variacionales.

Por ello, en este capítulo vamos a definir algunos conceptos elementales sobre sistemas hamiltonianos y mapas simplécticos, que son necesarios para la comprensión de las herramientas que se utilizarán en este trabajo de tesis. Luego, presentaremos las principales características de los ICs que han sido implementados en la herramienta previamente mencionada, que fueron necesarios para detectar las regiones caóticas de nuestro sistema de estudio y el análisis presentado en el Capítulo 6. 


\section{I CONCEPTOS BÁSICOS}

\subsubsection{Sistema dinámico}

En [79] se define un sistema dinámico como un sistema físico y/o matemático que presenta dos características:

- Un conjunto de $K$ variables de estado reales $x_{1}, x_{2}, \ldots, x_{K}$, cuyos valores definen el estado del sistema.

- Una regla bien definida de la cual se puede derivar la evolución del estado con respecto a una variable real independiente (la cual es usualmente referida como el tiempo $\mathrm{t}$ ). Llamamos al número $\mathrm{K}$ de variables de estado la dimensión del sistema, y denotamos un estado utilizando el vector $\mathbf{x}=\left(x_{1}, x_{2}, \ldots, x_{K}\right)$. Un estado $\mathbf{x}$ particular corresponde a un punto del espacio $\mathrm{K}$-dimensional $\mathcal{S}$, llamado espacio de fases del sistema, mientras que a un conjunto de estados $\mathbf{x}(\mathrm{t})$ parametrizados por $\mathrm{t}$ lo llamamos órbita $\mathrm{o}$ trayectoria del sistema dinámico.

Los sistemas dinámicos se pueden clasificar esencialmente en dos tipos: continuos y discretos.

\subsubsection{Sistemas dinámicos continuos - sistemas hamiltonianos}

Un sistema dinámico continuo está descripto por un conjunto de ecuaciones diferenciales de la forma

$$
\dot{\mathbf{x}}=\frac{\mathrm{d} \mathbf{x}}{\mathrm{dt}}=\mathbf{f}(\mathbf{x}, \mathrm{t}),
$$

donde $f$ es un conjunto de $K$ funciones $f_{1}, f_{2}, \ldots, f_{K}$.

Un sistema hamiltoniano es un caso particular de sistema dinámico continuo. Si este tipo de sistema es conservativo (es decir, que no depende explícitamente de $t$ ) de $l$ grados de libertad (lD, con $K=2 l$ ), será descripto por un Hamiltoniano

$$
H\left(q_{1}, q_{2}, \ldots, q_{l}, p_{1}, p_{2}, \ldots, p_{l}\right)=h,
$$

donde $q_{i}$ y $p_{i}$ con $i=1, \ldots, l$ son las coordenadas y momentos generalizados, respectivamente, $y \mathrm{~h}$ es una constante. Una órbita en la superficie de energía $\mathcal{M}_{h}$, de dimensión $2 l-1$, es descripta por un vector

$$
x(t)=\left(q_{1}(t), q_{2}(t), \ldots, q_{l}(t), p_{1}(t), p_{2}(t), \ldots, p_{l}(t)\right),
$$


donde $x_{i}=q_{i}$ y $x_{i+l}=p_{i}$, con $i=1, \ldots, l$ satisfacen la Ec. (2.2). La evolución temporal de esta órbita está regida por las ecuaciones de Hamilton que, en su forma matricial, se escriben como

$$
\dot{\mathbf{x}}=\mathbf{f}(\mathbf{x})=\left[\begin{array}{ll}
\frac{\partial H}{\partial \mathbf{p}} & -\frac{\partial H}{\partial \mathbf{q}}
\end{array}\right]^{\top}=\mathbf{J}_{2 l} \cdot \mathbf{D H},
$$

$$
\operatorname{con} \mathbf{q}=\left(q_{1}(t), q_{2}(t), \ldots, q_{l}(t)\right), p=\left(p_{1}(t), p_{2}(t), \ldots, p_{l}(t)\right), y
$$

$$
\mathbf{D H}=\left[\frac{\partial H}{\partial q_{1}} \frac{\partial H}{\partial q_{2}} \ldots \frac{\partial H}{\partial q_{l}} \frac{\partial H}{\partial p_{1}} \frac{\partial H}{\partial p_{2}} \ldots \frac{\partial H}{\partial p_{l}}\right]^{\top} .
$$

La matriz $\mathbf{J}_{2 l}$ posee la siguiente forma por bloques

$$
\mathbf{J}_{2 \mathrm{l}}=\left(\begin{array}{cc}
\mathbf{o}_{\mathrm{l}} & \mathbf{I}_{\mathrm{l}} \\
-\mathbf{I}_{\mathrm{l}} & \mathbf{o}_{\mathrm{l}}
\end{array}\right),
$$

donde $\mathbf{I}_{l}$ y $\mathbf{o}_{l}$ son la matriz identidad y la matriz nula $l \times l$, respectivamente.

Formalmente, la evolución de una órbita en un sistema dinámico continuo se expresa mediante

$$
\mathbf{x}(\mathrm{t})=\boldsymbol{\Phi}^{\mathrm{t}}(\mathbf{x}(0)),
$$

donde $\Phi^{\mathrm{t}}: \mathcal{S} \rightarrow \mathcal{S}$ es una función que transforma un punto inicial $\mathbf{x}(0) \in \mathcal{S}$ a un punto definido a un tiempo $t, \mathbf{x}(t)$ también perteneciente a $\mathcal{S}$.

\subsubsection{Ecuaciones variacionales}

Nuestro interés está focalizado en el estudio de los ICs variacionales, los cuales se construyen a partir de la evolución de la solución de los llamados vectores desviación. Estos vectores dan una medida de las desviaciones con respecto a una órbita de referencia, y los denotamos como w. Su evolución, por otro lado, está regida por las llamadas ecuaciones variacionales primeras.

Veamos ahora la evolución de los vectores desviación $\mathbf{w}$ para una dada órbita de referencia en un sistema hamiltoniano. Esta evolución será la base del estudio de los ICs que veremos más adelante. Dichos vectores evolucionan en el espacio tangente $\mathcal{T}_{\mathbf{x}} \mathcal{S}$ de $\mathcal{S}$ ([79]). Denotemos como $\mathrm{d}_{\mathbf{x}} \boldsymbol{\Phi}^{\mathrm{t}}$ al mapa lineal que transforma el espacio tangente de $\mathcal{S}$ en el punto $\mathbf{x}$ al espacio tangente en el punto $\Phi^{\mathrm{t}}(\mathbf{x})$, obteniendo $\mathrm{d}_{\mathbf{x}} \boldsymbol{\Phi}^{\mathrm{t}}: \mathcal{T}_{\mathbf{x}} \mathcal{S} \rightarrow \mathcal{T}_{\boldsymbol{\Phi}^{\mathrm{t}}(\mathbf{x})} \mathcal{S}$ con

$$
\mathbf{w}(\mathrm{t})=\mathrm{d}_{\mathbf{x}} \Phi^{\mathrm{t}} \mathbf{w}(0)
$$


donde $\mathbf{w}_{0}, \mathbf{w}$ son vectores desviación con respecto a la órbita dada a tiempos $\mathrm{t}=0 \mathrm{y} \mathrm{t}>0$, respectivamente.

En el caso de un sistema hamiltoniano, un vector desviación inicial

$$
\mathbf{w}(0)=\left(\delta x_{1}(0), \delta x_{2}(0), \ldots, \delta x_{2 l}(0)\right)
$$

de la órbita $\mathbf{x}(\mathrm{t})$ (Ec. (2.7)) evoluciona en un espacio tangente $\mathcal{T}_{\mathbf{x}} \mathcal{S}$ de acuerdo a las llamadas ecuaciones variacionales primeras ([79])

$$
\dot{\mathbf{w}}=\mathbf{D f}(\mathbf{x}(t)) \cdot \mathbf{w}=\frac{\partial \mathbf{f}}{\partial \mathbf{x}}(\mathbf{x}(t)) \cdot \mathbf{w}=\left[\mathbf{J}_{2 l} \cdot \mathbf{D}^{2} \mathbf{H}(\mathbf{x}(t))\right] \cdot \mathbf{w},
$$

con $\mathbf{D}^{2} \mathbf{H}(\mathbf{x}(\mathrm{t}))$ la matriz hessiana del hamiltoniano calculada para la órbita de referencia de la Ec. (2.7), es decir

$$
\mathbf{D}^{2} \mathbf{H}(\mathbf{x}(t))_{i, j}=\left.\frac{\partial^{2} H}{\partial x_{i} \partial x_{j}}\right|_{\mathbf{x}(t)} \quad, i, j=1,2, \ldots, K
$$

\subsubsection{Sistemas dinámicos discretos - Mapas}

En este trabajo de tesis nos dedicaremos principalmente al estudio de sistemas hamiltonianos discretos, llamados mapas. Éstos están descriptos por ecuaciones de diferencias de la forma

$$
\mathbf{x}_{\mathrm{n}+1}=\mathbf{f}\left(\mathbf{x}_{\mathrm{n}}\right),
$$

donde $\mathbf{f}$ es una función vectorial de $K$ dimensiones, es decir $\mathbf{f}=\left(f_{1}, f_{2}, \ldots, f_{K}\right)$ $\mathrm{y} \mathbf{x}$ es un vector a un tiempo discreto $t=n$ (entero).

Un mapa simpléctico es un mapa que preserva el volumen, cuya matriz jacobiana

$$
\mathbf{M}=\mathbf{D f}(\mathbf{x})=\frac{\partial \mathbf{f}}{\partial \mathbf{x}}=\left(\begin{array}{cccc}
\frac{\partial f_{1}}{\partial x_{1}} & \frac{\partial f_{1}}{\partial x_{2}} & \cdots & \frac{\partial f_{1}}{\partial x_{K}} \\
\frac{\partial f_{2}}{\partial x_{1}} & \frac{\partial f_{2}}{\partial x_{2}} & \cdots & \frac{\partial f_{2}}{\partial x_{K}} \\
\vdots & \vdots & & \vdots \\
\frac{\partial f_{K}}{\partial x_{1}} & \frac{\partial f_{K}}{\partial x_{2}} & \cdots & \frac{\partial f_{K}}{\partial x_{K}}
\end{array}\right)
$$

satisface

$$
\mathbf{M}^{\top} \cdot \mathbf{J}_{\mathrm{K}} \cdot \mathbf{M}=\mathbf{J}_{\mathrm{K}} \cdot
$$


El estado del sistema al tiempo discreto $t=n$ es dado por

$$
\mathbf{x}_{\mathrm{n}}=\boldsymbol{\Phi}^{\mathrm{n}}\left(\mathbf{x}_{0}\right)=(\mathbf{f})^{\mathrm{n}}\left(\mathbf{x}_{0}\right),
$$

donde $(\mathbf{f})^{\mathfrak{n}}\left(\mathbf{x}_{0}\right)=\mathbf{f}\left(\mathbf{f}\left(\ldots\left(\mathbf{f}\left(\mathbf{x}_{0}\right)\right)\right)\right.$, es la aplicación de la función $\mathbf{f}$ sobre el vector $x_{0} n$ veces.

\subsubsection{Mapa tangente}

En un mapa simpléctico, la evolución de un vector desviación $\mathbf{w}_{n}$ con respecto a una órbita de referencia $\mathbf{x}_{n}$ está dada por el mapa tangente correspondiente ([79])

$$
\mathbf{w}_{n+1}=\operatorname{Df}\left(\mathbf{x}_{n}\right) \cdot \mathbf{w}_{n} .
$$

Por lo tanto, la evolución del vector desviación inicial $\mathbf{w}_{0}$ está dada por

$$
\mathbf{w}_{\mathrm{n}}=\mathbf{M}_{\mathrm{n}-1} \cdot \mathbf{M}_{\mathrm{n}-2} \cdot \ldots \cdot \mathbf{M}_{0} \cdot \mathbf{w}_{\mathrm{0}}=\mathbf{Z}_{\mathrm{n}} \cdot \mathbf{w}_{0},
$$

con $\mathbf{Z}_{\mathrm{n}}$ cumpliendo la relación

$$
\mathbf{Z}_{\mathrm{n}+1}=\mathbf{M}_{\mathrm{n}} \cdot \mathbf{Z}_{\mathrm{n}}=\mathbf{D f}\left(\mathbf{x}_{\mathrm{n}}\right) \cdot \mathbf{Z}_{\mathrm{n}}
$$

$\operatorname{con} \mathbf{Z}_{0}=\mathbf{I}_{\mathrm{K}}$.

Tanto para el caso discreto como para el caso continuo, las ecuaciones variacionales (que se corresponde con el mapa tangente para el caso discreto) deben resolverse junto con las ecuaciones de movimiento (ecuaciones del mapa para el caso discreto) ya que, por lo general, las ecuaciones variacionales dependen también de las coordenadas en el espacio de fases de la órbita.

La evolución de los vectores desviación mediante la solución de las ecuaciones variacionales es la base sobre la cual se definen los indicadores que veremos en este capítulo (que se los puede denominar como indicadores variacionales debido al origen de su definición).

\subsubsection{Definición de caos}

Una vez definido lo que es un sistema dinámico, debemos ver lo que se entiende por el término caos. 
El caos puede definirse de forma sencilla como: "la sensibilidad que tiene una trayectoria ante un cambio en las condiciones iniciales, por más pequeño que éste sea".

En [69], se hace referencia a este fenómeno como: "Pequeños errores en las condiciones iniciales producen enormes diferencias en los resultados". Esto se puede entender como sigue. Supongamos que tenemos una dada condición inicial, la cual describirá una trayectoria cuando evoluciona con el tiempo. Si variamos levemente esta condición inicial, al cabo de cierto tiempo su trayectoria divergirá exponencialmente con respecto a la trayectoria original.

\subsection{DEFINICIONES DE LOS INDICADORES DE CAOS}

Para realizar el estudio de diversos fenómenos dinámicos de nuestro interés en un sistema, éste debe contar con un espacio de fases dividido ${ }^{1}$, debido a que las condiciones iniciales sólo pueden difundir a través de la componente caótica del sistema ([38], [39], [85]).

Conocer la estructura caótica de un sistema es de vital importancia antes de proceder a realizar dichos estudios. Para esto es necesario contar con una herramienta que permita identificar las regiones donde se manifiesta el caos. Dichas herramientas nos permiten tener conocimiento de la estructura dinámica del sistema.

Para llevar a cabo este propósito, como se ha mencionado al comienzo de este capítulo, conjuntamente con el Dr. Maffione se diseñó un código, llamado LP-VIcode, que implementa de manera eficiente un paquete de ICs, basados en la variación de la solución de las ecuaciones variacionales, el cual cuenta con varias propiedades que serán descriptas en el Capítulo 3. Dichas propiedades lo hacen una herramienta muy útil y versátil al momento de estudiar el espacio de fases de un sistema, ya sea un mapa simpléctico o un flujo hamiltoniano.

Este programa cuenta con dos versiones: una para el estudio de mapas simplécticos, como el vFSM ([19], [78], [80]) cuyo nombre significa que es una variante del mapa simpléctico de Froeschlé ([33]), o el CRSSM ([17]) y otra versión para el estudio de flujos hamiltonianos, como el potencial de [45] o el correspondiente a un modelo de galaxia elíptica triaxial ([63]).

Veamos a continuación una breve descripción de los indicadores hasta ahora implementados en el programa.

I Se llama espacio de fases dividido a un espacio de fases donde coexisten simultáneamente órbitas regulares y caóticas. 


\subsubsection{El Indicador de Lyapunov $(L I)^{2}$}

Los Exponentes Característicos de Lyapunov (LCEs, del inglés Lyapunov Characteristic Exponents) son cantidades asintóticas que caracterizan la tasa promedio de crecimiento (o disminución) de la norma de los vectores desviación de las órbitas de un sistema dinámico. Este concepto fue introducido por Lyapunov en 1892 para el estudio de la estabilidad de la solución de ecuaciones diferenciales ordinarias ([54]). Como ya hemos mencionado, una de las características elementales del caos es la sensibilidad en la variación de las condiciones iniciales, y los LCEs nos dan una medida cuantitativa de la sensibilidad de un sistema dinámico a pequeños cambios en éstas. Para un sistema de $l$ grados de libertad tendremos $2 l$ LCEs: $l$ LCEs positivos y sus l opuestos ([6]). Si al menos uno de ellos es positivo, entonces la órbita es caótica, ya que como veremos enseguida, el exponente positivo es un indicador de que las órbitas se están alejando exponencialmente en esa dirección. En cambio, si todos ellos son iguales a 0 , entonces la órbita será regular.

Consideremos un sistema dinámico (continuo o discreto) sobre una variedad diferenciable $\mathcal{S}$, donde $\Phi^{\mathrm{t}}(\mathbf{x}(0))=\mathbf{x}(\mathrm{t})$ caracteriza el estado del sistema al tiempo $t$, siendo $\mathbf{x}(0)=\mathbf{x}_{0}$ el estado del sistema al tiempo $t=0$ (Ec. (2.7) o (2.14)). El estado del sistema luego de dos intervalos de tiempo consecutivos $t$ y $t^{\prime}$ será dado por la ley de composición: $\boldsymbol{\Phi}^{\mathrm{t}+\mathrm{t}^{\prime}}=\boldsymbol{\Phi}^{\mathrm{t}} \circ \boldsymbol{\Phi}^{\mathrm{t}^{\prime}}$.

Recordemos que el espacio tangente a $\mathbf{x}$ se transforma en el espacio tangente a $\boldsymbol{\Phi}^{\mathrm{t}}(\mathbf{x})$ mediante el operador $\mathrm{d}_{\mathbf{x}} \boldsymbol{\Phi}^{\mathrm{t}}$ (Ec. (2.8)). La acción de dicho operador a intervalos de tiempo consecutivos satisfacen la ecuación:

$$
\mathrm{d}_{\mathbf{x}} \Phi^{\mathrm{t}+\mathrm{t}^{\prime}}=\mathrm{d}_{\Phi^{\mathrm{t}^{\prime}}(\mathbf{x})} \Phi^{\mathrm{t}} \circ \mathrm{d}_{\mathbf{x}} \Phi^{\mathrm{t}^{\prime}} .
$$

Si suponemos que nuestra variedad $\mathcal{S}$ tiene cierta norma denotada por $\|\cdot\|$, podemos definir la cantidad:

$$
\lambda_{\mathrm{t}}(\mathbf{x})=\frac{\left\|\mathrm{d}_{\mathbf{x}} \boldsymbol{\Phi}^{\mathrm{t}} \mathbf{w}\left(\mathrm{t}_{0}\right)\right\|}{\left\|\mathbf{w}\left(\mathrm{t}_{0}\right)\right\|}
$$

llamada "factor de crecimiento" en la dirección de $\mathbf{w}\left(t_{0}\right)$. Si

$$
\limsup _{t \rightarrow \infty} \frac{1}{t} \ln \left(\lambda_{t}(\mathbf{x})\right)>0
$$

2 El contenido de esta sección fue tomado de [79]. 
entonces decimos que la órbita tiene una divergencia exponencial en la dirección de $\mathbf{w}$.

Generalmente, para identificar una órbita como caótica o regular, basta sólo con estudiar el máximo LCE, llamado ILCE (del inglés largest LCE). Esto es debido a que, si el lLCE $>0$ la órbita es caótica, independientemente del valor de los otros LCE. Por otro lado, si $\operatorname{LCCE}=0$, como es el máximo, el resto de los LCE también serán 0 , obteniendo una órbita regular.

En el LP-VIcode hemos implementado la variante a tiempo finito del ILCE como se encuentra definida en [7] y [6], llamado LI (del inglés Lyapunov Indicator). Esta implementación solamente evalúa el máximo exponente (lLCE), convirtiéndolo en un IC más rápido que si calculamos los $2 l$ LCE.

\subsubsection{El Indicador Relativo de Lyapunov (RLI)}

En [73] los autores calcularon el $L I$ en distintas regiones $\mathbf{x}_{0}$ del problema restringido de los tres cuerpos, luego de j pasos de integración (o iteración, según se considere el caso continuo o discreto), que llamaremos $\operatorname{LI}\left(\mathbf{x}_{0} ; \mathbf{j}\right)$. Encontraron que, para regiones de movimiento regular, la variación de $\operatorname{LI}\left(\mathbf{x}_{0} ; \mathbf{j}\right)$ con $\mathbf{x}_{0}$ es muy suave, mientras que en las regiones caóticas se observan grandes fluctuaciones. En regiones débilmente caóticas, sin embargo, dicha fluctuación no era suficiente como para lograr una clasificación clara. Es por esto que los autores comenzaron a estudiar la evolución temporal de esta fluctuación con lo cual, para amplificarlas, calculan el $L I$ para dos órbitas muy próximas entre sí, es decir

$$
\Delta \operatorname{LI}\left(\mathbf{x}_{0}(t)\right)=\left\|\operatorname{LI}\left(\mathbf{x}_{0}+\Delta \mathbf{x} ; t\right)-\operatorname{LI}\left(\mathbf{x}_{0} ; t\right)\right\|,
$$

donde $\mathbf{x}_{0} \mathbf{y}_{\mathbf{x}_{0}}+\Delta \mathbf{x}$ son dos condiciones iniciales muy cercanas a tiempo $t$, separadas por una cantidad $\Delta \mathbf{x}$, que es un parámetro libre. Luego, el $R L I$ (del inglés Relative Lyapunov Indicator) es definido como un promediado ([74]) a través de la expresión:

$$
\operatorname{RLI}(t)=<\Delta \operatorname{LI}\left(\mathbf{x}_{0}\right)>_{t}=\frac{1}{t} \sum_{i=1}^{t / \delta t} \Delta \operatorname{LI}\left(\mathbf{x}_{0} ; i \times \delta \mathrm{t}\right),
$$

con $i$ el número de pasos de integración (o iteración) de tamaño $\delta$ t.

La cantidad $\Delta \mathbf{x}$ es, en principio, arbitraria, y por ello un parámetro libre de nuestro código. Pero, al momento de definir su valor, debemos hacerlo con cuidado dado que, si elegimos un valor que no sea lo suficientemente pequeño 
podríamos encontrarnos con órbitas que están muy próximas espacialmente, pero que pertenecen a dos dominios dinámicos completamente diferentes. Es por eso que en [73] los autores recomiendan que los valores para $\Delta \mathbf{x}$ cumplan $\|\Delta \mathbf{x}\| \in\left[10^{-14}, 10^{-7}\right]$ para reducir la probabilidad de que esto ocurra.

Con el valor de $\Delta \mathbf{x}$ elegido, los valores del $R L I$ obtenidos para órbitas regulares son varios órdenes de magnitud menores que los valores del RLI para órbitas caóticas, facilitando la clasificación entre estos dos comportamientos.

\subsubsection{Los Espectros Dinámicos de los Números de Dilatación Local (SSNs) y la Dis-} tancia Espectral (DS)

El número de dilatación local SN (del inglés Stretching Number) $s_{i}$ está definido como ([86]):

$$
s_{i}=\frac{1}{\delta t} \ln \frac{\|\mathbf{w}(t+i \times \delta t)\|}{\|\mathbf{w}(t+(i-1) \times \delta t)\|},
$$

donde $d_{x} \Phi^{t+i \times \delta t}(\mathbf{w}(0))=\mathbf{w}(t+i \times \delta t)$ es el vector desviación al tiempo $t+$ $i \times \delta t$, con $i=1,2, \ldots, p$ y $p$ es la cantidad de pasos de integración $\delta$ t. En el caso de mapas, tenemos que $\delta \mathrm{t} \equiv 1$. El espectro de los $s_{i}$, llamado SSN (del inglés Spectra of the Stretching Numbers), posee más información que el LI. Por ejemplo, en [20] los autores mostraron que dos órbitas caóticas pueden tener ambas el mismo LI pero sus espectros ser completamente diferentes. Es por esto que los SSNs también son una herramienta muy útil ya que, a partir de observar las diferencias entre los dos espectros, podemos fácilmente clasificar la órbita como regular o caótica.

Los SSNs son dados por la densidad de probabilidad de los valores $s$ dados por el $s_{i}$, es decir

$$
S(s) d s=\frac{d N(s)}{N}
$$

donde $\mathrm{N}$ es el número total de $s_{i}$ y $\mathrm{dN}(\mathrm{s})$ es el número de $s_{i}$ en el intervalo $(s, s+d s)$. Por esto, el cálculo de los SSNs en el LP-VIcode es simplemente la construcción de sus histogramas.

Hay dos propiedades de los $S S N$ s que resultan muy útiles cuando estamos identificando órbitas caóticas ([87]):

1. El espectro $S(s)$ es invariante con respecto a la condición inicial que se tome a lo largo de la órbita (invariante temporal).

2. El espectro $S(\mathrm{~s})$ es invariante con respecto a la condición inicial y al vector desviación inicial si pertenecen a un dominio caótico conectado, es decir, que estén caracterizados por el mismo lLCE (invariante espacial). 
En contrapartida, si tenemos un conjunto muy grande de órbitas, el cálculo de los espectros para cada una de ellas sería una tarea muy costosa en cuanto a tiempos computacionales. Es por esto que se introdujo el concepto de la distancia espectral DS ([87]), que podría solucionar este inconveniente. Esto se lleva a cabo mediante el cálculo de las diferencias entre los SSNs asociados a distintos vectores desviación iniciales, logrando una detección mucho más rápida de las órbitas caóticas, como mencionamos más adelante. Luego, basándose en esta idea, se define la distancia espectral $D S$ como la diferencia de dos histogramas de una dada órbita. Esto es

$$
\mathrm{DS}^{2}=\sum_{\mathrm{k}}\left[\mathrm{S}_{1}\left(\mathrm{~s}_{\mathrm{k}}\right)-\mathrm{S}_{2}\left(\mathrm{~s}_{\mathrm{k}}\right)\right]^{2} \times \Delta \mathrm{s}
$$

donde $S_{j}(s)$ es el número de $s_{i}$ normalizado asociado al vector desviación inicial $\mathbf{w}_{\mathbf{j}}(0)$, el cual tiene valores en el intervalo $\left(s_{k}, s_{k}+\Delta s\right)$. Para una órbita regular, los espectros serán bien diferentes, lo que produce un valor de $D S$ distinto de cero. En cambio, si las órbitas son caóticas, sus espectros se parecerán, y $D S$ tenderá a cero.

La implementación del SSN y del DS en el LP-VIcode está basada en el trabajo de [87], que se reduce a la implementación de las Ecs. (2.20), (2.21) y (2.22).

\subsubsection{El Factor de Crecimiento Exponencial Medio entre Órbitas Cercanas (MEGNO)}

A continuación veremos las principales características del MEGNO (cuyo nombre proviene de su acrónimo en inglés Mean Exponential Growth Factor of Nearby Orbits). Para mayores detalles ver [17].

Sea $\mathrm{H}(\mathbf{p}, \mathbf{q})$ con $\mathbf{p}, \mathbf{q} \in \mathbb{R}^{\mathrm{l}}$ un hamiltoniano de $\mathrm{l}$ grados de libertad el cual, para simplificar las ecuaciones y la explicación, supondremos que no depende explícitamente del tiempo. Recordemos entonces que:

$$
\mathbf{x}=(\mathbf{p}, \mathbf{q}) \in \mathbb{R}^{2 l}, \mathbf{f}(\mathbf{x})=\left[\frac{\partial \mathrm{H}}{\partial \mathbf{p}}-\frac{\partial \mathrm{H}}{\partial \mathbf{q}}\right]^{\top} \in \mathbb{R}^{2 l},
$$

donde las ecuaciones de movimiento vienen dadas por:

$$
\dot{\mathbf{x}}=\mathbf{f}(\mathbf{x}) .
$$

El ILCE mide la tasa local media de divergencia exponencial entre órbitas cercanas. Esto se ve claramente cuando escribimos la Ec. (2.18) en su forma integral,

$$
\chi\left(\mathbf{x}_{0}\right)=\lim _{\mathrm{t} \rightarrow \infty} \frac{1}{\mathrm{t}} \int_{0}^{\mathrm{t}} \frac{\left\|\dot{\mathrm{d}}_{\mathbf{x}} \boldsymbol{\Phi}^{\mathrm{t}^{\prime}} \mathbf{w}_{\mathrm{O}}\right\|}{\left\|\mathrm{d}_{\mathbf{x}} \boldsymbol{\Phi}^{\mathrm{t}^{\prime}} \mathbf{w}_{\mathrm{O}}\right\|} \mathrm{dt}^{\prime}=\overline{\left(\frac{\left\|\dot{\mathrm{d}}_{\mathbf{x}} \boldsymbol{\Phi}^{\mathrm{t}^{\prime}} \mathbf{w}_{\mathrm{O}}\right\|}{\left\|\mathrm{d}_{\mathbf{x}} \boldsymbol{\Phi}^{\mathrm{t}} \mathbf{w}_{\mathrm{O}}\right\|}\right)},
$$


donde la barra denota un promediado en el tiempo.

Ahora podemos introducir la cantidad $Y\left(\mathbf{x}_{0}, t\right)$, por medio de la expresión:

$$
\mathrm{Y}\left(\mathbf{x}_{0}, \mathrm{t}\right)=\frac{2}{\mathrm{t}} \int_{0}^{\mathrm{t}} \frac{\left\|\dot{\mathrm{d}}_{\mathbf{x}} \boldsymbol{\Phi}^{\mathrm{t}^{\prime}} \mathbf{w}_{0}\right\|}{\left\|\mathrm{d}_{\mathbf{x}} \boldsymbol{\Phi}^{\mathrm{t}^{\prime}} \mathbf{w}_{0}\right\|} \mathrm{t}^{\prime} \mathrm{dt} \mathrm{t}^{\prime}
$$

que está relacionada con la integral en la Ec. (2.24); es decir, en caso de un incremento exponencial de $\left\|d_{\mathbf{x}} \boldsymbol{\Phi}^{\mathrm{t}} \mathbf{w}\right\|$, entonces $Y\left(\mathbf{x}_{0}, t\right)$ puede ser considerada como una variante pesada de la integral de la Ec. (2.24).

Luego, definimos al MEGNO como el promedio temporal de Y:

$$
\bar{Y}\left(x_{0}, t\right) \equiv \frac{1}{t} \int_{0}^{t} Y\left(x_{0}, t^{\prime}\right) d t^{\prime} .
$$

El comportamiento asintótico del MEGNO puede ser descripto en forma resumida de la siguiente manera:

$$
\bar{Y}\left(x_{0}, t\right) \approx a t+b,
$$

donde $\mathrm{a}=\chi / 2 \mathrm{y} \mathrm{b} \approx 0$ para movimiento irregular, caótico; mientras que $\mathrm{a}=0$ y $\mathrm{b} \approx 2$ para movimiento condicionalmente periódico (para movimiento periódico: $a=0$ y $b \approx 0$ ). Pequeñas desviaciones del valor $b=2$ indican que la trayectoria pasa cerca de objetos particulares del espacio de fases, siendo $\mathrm{b} \lesssim 2 \mathrm{o} b \gtrsim 2$ cuando estos objetos son órbitas periódicas estables, $\mathrm{u}$ órbitas periódicas inestables, respectivamente (véase [17] para más detalles).

\subsubsection{Generalización del MEGNO}

Podemos generalizar el $Y\left(\mathbf{x}_{0}, t\right)$, Ec. (2.25), de la siguiente manera ([17]):

$$
Y_{m, n}\left(x_{0}, t\right)=(m+1) \cdot t^{n} \int_{0}^{t} \frac{\left\|\dot{d}_{x} \Phi^{t^{\prime}} \mathbf{w}\right\|}{\left\|d_{x} \Phi^{t^{\prime}} \mathbf{w}\right\|}\left(t^{\prime}\right)^{m} d t^{\prime},
$$

y se define el MEGNO generalizado como:

$$
\bar{Y}_{m, n}\left(x_{0}, t\right) \equiv \frac{1}{t^{m+n+1}} \int_{0}^{t} Y_{m, n}\left(x_{0}, t^{\prime}\right) d t^{\prime}
$$

El comportamiento asintótico de $\bar{Y}_{\mathrm{m}, \mathrm{n}}\left(\mathrm{x}_{0}, \mathrm{t}\right)$ puede ser también resumido como lo expresa la Ec. (2.27), donde ahora $a=\chi_{i} /(m+n+2)$ y $b \approx 0$ para movimiento irregular, caótico y $a=0$ y $b \approx(m+1) /(m+n+1)$ para movimiento estable, condicionalmente periódico. 
La principal ventaja que presenta la posibilidad de elegir los valores $(m, n)$, es que permite incrementar la velocidad de convergencia del MEGNO, pero este incremento hace que también aumenten las fluctuaciones en su evolución. Dado que de aquí en más se va a utilizar siempre el MEGNO generalizado, lo llamaremos simplemente MEGNO.

Para el caso de mapas, el MEGNO se define de manera análoga a la definición para sistemas continuos, reemplazando las integrales con respecto a t por sumas con respecto al número de iteraciones, por tratarse de un sistema discreto. Asimismo, para el cálculo variacional, utilizamos el mapa tangente en reemplazo de las ecuaciones variacionales. Luego, las Ecs. (2.28) y (2.29) adoptan la forma

$$
\begin{aligned}
& Y_{m, n}(N)=(m+1) N^{n} \sum_{k=1}^{N} \ln \left(\frac{\left\|\mathbf{w}_{k}\right\|}{\left\|\mathbf{w}_{k-1}\right\|}\right) k^{m}, \\
& \bar{Y}_{m, n}(N)=\frac{1}{N^{m+n+1}} \sum_{k=1}^{N} Y_{m, n}(k)
\end{aligned}
$$

con $m, n \in \mathbb{Z}$.

Con esta expresión (o con la equivalente para flujos hamiltonianos, podemos definir diferentes valores del MEGNO para distintas combinaciones del par $(m, n)$. En este trabajo, para el caso de flujos hamiltonianos utilizaremos la variante $(m, n)=(1,-1)$, que tiende asintóticamente a 2 para órbitas regulares, y para el caso de mapas adoptamos la versión $(m, n)=(2,0)$, la cual tiende a 0,5 para este tipo de órbitas.

2.2.5 El Índice Menor de Alineamiento (SALI) y el Índice de Alineamiento Generalizado (GALI)

En [78] introducen el SALI (del inglés Smaller Alignment Index) de la siguiente manera: a partir de dos vectores desviación para un dado tiempo $t, \mathbf{w}_{1}(t) y$ $\mathbf{w}_{2}(t)$, definen los índices paralelo y antiparalelo

$$
\begin{aligned}
& \mathrm{d}_{-}=\left\|\mathbf{w}_{1}(\mathrm{t})-\mathbf{w}_{2}(\mathrm{t})\right\| \\
& \mathrm{d}_{+}=\left\|\mathbf{w}_{1}(\mathrm{t})+\mathbf{w}_{2}(\mathrm{t})\right\|,
\end{aligned}
$$

respectivamente, con $\|\cdot\|$ la norma euclideana. Luego, definen el SALI a un tiempo $t$ como el menor de estos dos índices:

$$
\operatorname{SALI}(\mathrm{t})=\operatorname{mín}\left(\mathrm{d}_{+}, \mathrm{d}_{-}\right) \text {. }
$$


En el LP-VIcode el cálculo del SALI está implementado de la manera descripta, excepto que el código normaliza, en cada paso, los vectores desviación $\mathbf{w}_{i}(t)$, con $i=1,2$. La razón de esto es que, ante la presencia de una órbita caótica, los vectores desviación $\mathbf{w}_{1}(\mathrm{t})$ y $\mathbf{w}_{2}(\mathrm{t})$ tienden a coincidir en su dirección, resultando o bien paralelos o bien antiparalelos. Si dichos vectores están normalizados, tendremos que $\mathrm{d}_{-}=0 \mathrm{y} \mathrm{d}_{+}=2$ en el caso en que los vectores resulten paralelos y $\mathrm{d}_{-}=2 \mathrm{y} \mathrm{d}_{+}=0$ en el caso contrario. En el caso de que nos encontremos con órbitas regulares, los valores de $d_{-} y d_{+}$oscilarán en el intervalo $(0 ; 2)$. Por lo tanto, el valor del $S A L I$ será nulo para órbitas caóticas y no nulo para órbitas regulares.

En [81] se define otro indicador, que sigue el mismo comportamiendo que el SALI, como

$$
\mathrm{P}(\mathrm{t})=\mathrm{d}_{-} \cdot \mathrm{d}_{+} \cdot
$$

En el caso de órbitas caóticas, uno de los dos índices tiende a 0 , haciendo que $P(t) \rightarrow 0$. En el caso de órbitas regulares ambos índices serán $\neq 0$, por lo que $\mathrm{P}(\mathrm{t})$ también lo será, y oscilará entre estos dos valores. Para el cálculo efectivo del SALI ellos utilizan

$$
\left\|\mathbf{w}_{1}(\mathrm{t}) \wedge \mathbf{w}_{2}(\mathrm{t})\right\|=\frac{\mathrm{P}(\mathrm{t})}{2},
$$

que sería el área del paralelepípedo formado por los vectores $\mathbf{w}_{1}(t)$ y $\mathbf{w}_{2}(t)$. En este trabajo no utilizaremos este método para calcular el $S A L I$ ya que el cómputo del producto vectorial es más costoso computacionalmente que utilizar las Ecs. (2.31) y (2.32).

Definido el SALI como en la Ec. (2.34) permitió desarrollar una versión generalizada de éste, que sirve para calcular un indicador en un caso general de l grados de libertad, llamado GALI (del inglés Generalized Alignment Index). En [81] los autores lo hacen mediante el cálculo del volumen del paralelepípedo formado por $k$ vectores desviación $\mathbf{w}_{1}(t), \mathbf{w}_{2}(t), \ldots, \mathbf{w}_{k}(t)$, con $2<k \leqslant 2 l$ mediante el producto vectorial

$$
\operatorname{GALI}_{k}(\mathrm{t})=\left\|\hat{\mathbf{w}}_{1}(\mathrm{t}) \wedge \hat{\mathbf{w}}_{2}(\mathrm{t}) \wedge \cdots \wedge \hat{\mathbf{w}}_{\mathrm{k}}(\mathrm{t})\right\|,
$$

donde se tiene que $\hat{\mathbf{w}}_{i}=\mathbf{w}_{i} /\left\|\mathbf{w}_{i}\right\|$, con $i=1, \ldots, k$.

Si bien el cálculo del $S A L I$ es sencillo (en términos computacionales), para el $G A L I_{k}$ ya no lo es dado que, para el cálculo de los productos vectoriales, necesitamos resolver una gran cantidad de determinantes a fin de calcular el volumen del paralelepípedo. Por ejemplo, para calcular el $G A L I_{8}$ se necesitan 
evaluar $1,287 \times 10^{4}$ determinantes de $8 \times 8$, y el $G A L I_{15}$ necesita de $1,5511752 \times$ $10^{8}$ determinantes de $15 \times 15$.

Dado que estos cálculos son muy costosos, en [82] utilizaron una variante para resolver este problema, la descomposición en valores singulares (SVD por sus siglas en inglés singular value decomposition) calculada de la forma

$$
\log \left(\mathrm{GALI}_{\mathrm{k}}\right)=\sum_{\mathrm{i}=1}^{\mathrm{k}} \log \left(z_{\mathrm{i}}\right)
$$

donde los $z_{i}$ son los valores singulares de la matriz singular $Z$.

Como hemos mencionado previamente, el LP-VIcode calcula el SALI siguiendo las Ecs. (2.31) y (2.32), y el GALI utilizando la rutina SVD de [70] para calcular el indicador a través de la Ec. (2.36).

\subsubsection{El Indicador Rápido de Lyapunov (FLI) y la componente Ortogonal del Indica- dor Rápido de Lyapunov (OFLI)}

El FLI (Fast Lyapunov Indicator) es una cantidad íntimamente relacionada con el LI ([34], [35], [36], [37], [43], [50], [57]). Es capaz de distinguir entre movimiento regular y caótico ([36], [37]) e incluso entre movimiento resonante y no resonante ([34], [43], [50]) utilizando solamente una parte del cálculo del LI.

Para un sistema l-dimensional, en el LP-VIcode podemos emplear dos algoritmos para calcular el FLI. Uno de ellos es a través de la evolución temporal de $2 l$ vectores desviación y, tiempo a tiempo, tomamos la norma euclideana de cada uno, almacenando la mayor de estas normas ([36]). Esto es, para un tiempo t, el FLI es calculado como

$$
\operatorname{FLI}(\mathrm{t})=\sup _{\mathrm{t}}\left[\left\|\mathbf{w}_{1}(\mathrm{t})\right\|,\left\|\mathbf{w}_{2}(\mathrm{t})\right\|, \ldots,\left\|\mathbf{w}_{2 l}(\mathrm{t})\right\|\right] .
$$

El otro mecanismo para el cálculo del FLI es el introducido en [32], que utiliza solamente un vector desviación.

Para una órbita caótica, el valor del FLI crece exponencialmente, mientras que para una órbita regular, también crece, pero a un ritmo mucho menor, presentando una dependencia de tipo lineal con el tiempo. Para la implementación numérica, se debe interrumpir el cálculo para un valor de saturación prefijado, para evitar desbordamiento.

Si bien el FLI y el MEGNO se han definido de manera independiente, existe una estrecha relación entre estos dos ICs, donde el último puede ser expresado en función del primero ([61]). 
En cuanto al OFLI ([32]), el concepto es similar al presentado para el FLI. La novedad que incluye este IC es que tomamos la norma de la componente ortogonal al flujo de cada vector desviación, tiempo a tiempo. Luego el OFLI es definido como

$$
\operatorname{OFLI}(t)=\sup _{t}\left[\left\|\mathbf{w}_{1}(t)^{\perp}\right\|,\left\|\mathbf{w}_{2}(t)^{\perp}\right\|, \ldots,\left\|\mathbf{w}_{2 l}(t)^{\perp}\right\|\right] .
$$

Una de las principales ventajas que presenta el OFLI con respecto al FLI, es que permite distinguir entre una órbita regular no periódica y una regular periódica, como se mostrará en la Sección 3.7.3.2.

Para el OFLI, al igual que para el FLI, podemos utilizar la versión que emplea $2 l$ vectores desviación como también la que utiliza solamente un vector desviación.

\subsubsection{La componente Ortogonal del Indicador Rápido de Lyapunov de segundo orden $\left(O F L I_{\mathrm{TT}}^{2}\right)$}

En la sección anterior hemos descripto el cálculo del FLI a partir de la solución de las ecuaciones variacionales de primer orden. También, tomando sólo la componente ortogonal de los vectores desviación, vimos como calcular el OFLI. El comportamiento de este último indicador es muy sensible a la elección de las condiciones iniciales, como se menciona en [1]. Es por esto que se sugiere ir más allá en el orden de las ecuaciones variacionales a resolver.

Es posible considerar un orden arbitrariamente alto de las ecuaciones variacionales pero, por lo general, no suele excederse de las ecuaciones variacionales de segundo orden, debido a un incremento notable en el costo computacional.

De esta manera, si llamamos $\mathbf{w}^{(1)}(t)$ al vector variacional de primer orden, el autor define la variante de segundo orden del OFLI para un tiempo final $t_{f}$ :

$$
\operatorname{OFLI}_{\mathrm{TT}}^{2}(\mathrm{t})=\sup _{0<\mathrm{t}<\mathrm{t}_{\mathrm{f}}} \hat{\mathbf{w}}(\mathrm{t})^{\perp}
$$

siendo $\hat{\mathbf{w}}(t)^{\perp}$ la componente ortogonal al flujo de $\hat{\mathbf{w}}(t)$, donde $\hat{\mathbf{w}}(t)$ se define como

$$
\hat{\mathbf{w}}(\mathrm{t})=\mathbf{w}^{(1)}(\mathrm{t})+\frac{1}{2} \mathbf{w}^{(2)}(\mathrm{t}),
$$

donde $\mathbf{w}^{(2)}(t)$ es la solución de las ecuaciones variacionales, a tiempo $t$, de segundo orden.

Como mencionamos más arriba, este cálculo se puede llevar a cabo hasta un orden arbitrariamente alto, simplemente extendiendo la definición de (2.39) 


$$
\operatorname{OFLI} \mathrm{TT}_{\mathrm{T}}(\mathrm{t})=\sup _{0<\mathrm{t}<\mathrm{t}_{\mathrm{f}}} \hat{\mathbf{w}}(\mathrm{t})^{\perp}
$$

tomando

$$
\hat{\mathbf{w}}(\mathrm{t})=\sum_{k=1}^{\mathrm{m}} \frac{1}{\mathrm{k} !} \mathbf{w}^{(\mathrm{k})}(\mathrm{t}) .
$$

Vemos que, para $k=2$, corresponde al caso de la Ec. (2.39). Para mayores detalles, consultar [1], [2], [4], [3].

\subsubsection{El Exponente Medio de Ley de Potencias (APLE)}

Este método, cuyo nombre proviene del inglés Average Power Law Exponent, está basado en el concepto de la Entropía de Tsallis, explicada en [53]. Aquí sólo nos limitaremos a mostrar los conceptos básicos para implementar el indicador en el LP-VIcode.

Supongamos un sistema de $l$ dimensiones. Para cada uno de los $l$ vectores desviación $\mathbf{w}_{j}(t)$ con $j=1, \ldots, l$, se define el índice $p_{j}$ mediante la expresión

$$
\mathbf{w}_{j}(t)=\mathbf{w}_{j}\left(t_{1}\right)\left(\frac{t}{t_{1}}\right)^{p_{j}}, j=1, \ldots, l .
$$

Sea $\mathbf{w}^{2}(t)=\sum_{j=1}^{l} \mathbf{w}_{j}{ }^{2}(t)$. Siguiendo el desarrollo en [53] se obtiene

$$
p=\frac{\ln \left(\frac{w^{2}(t)}{w^{2}\left(t_{1}\right)}\right)}{2 \ln \left(\frac{t}{t_{1}}\right)} .
$$

Dado que $t_{1}$ es un valor constante, $\mathbf{w}\left(t_{1}\right)$ también lo es. Por ende, el valor que tome $t_{1}$ no alterará la forma en la que el indicador clasifica las órbitas. Por ello, tomando $t_{1}=1$, la expresión se simplifica, y obtenemos el valor implementado del APLE

$$
\operatorname{APLE}=\frac{\ln (\|\mathbf{w}(\mathrm{t})\|)}{\ln (\mathrm{t})} .
$$

En el capítulo siguiente presentaremos el programa diseñado, el LP-VIcode, junto con algunos de los resultados de su implementación. 
UN CÓDIGO PARA CALCULAR ICS: EL LP-VICODE

Como ya hemos mencionado en el Capítulo 2, para cualquier estudio dinámico de un sistema (ya sean mapas simplécticos o flujos hamiltonianos) es esencial el uso de indicadores de caos (como, por ejemplo, en [17], [36], [44], [78], [79]), para tener una información más detallada sobre las regiones regulares y caóticas de un sistema. En este trabajo nos hemos centrado en el grupo de los indicadores variacionales.

Existe una extensa lista de ICs en la literatura, de los cuales muy pocos de ellos son utilizados por los investigadores abocados al estudio de la dinámica. Este fue el principal motivo del diseño de un código, llamado LP-VIcode y escrito en FORTRAN, que calcula de una manera eficiente una gran cantidad de estos ICs. El nombre proviene de su acrónimo en inglés La Plata Variational Indicators code.

En este capítulo presentaremos las principales características del LP-VIcode, tanto de su versión para mapas simplécticos como de su versión para flujos hamiltonianos. Luego mostraremos algunos resultados de su aplicación.

\subsection{CARACTERÍSTICAS GENERALES DEL LP-VICODE}

Este programa, escrito en FORTRAN77, fue introducido por primera vez en [23], y también ha sido utilizado en la realización de los cálculos que forman parte de [24],[56], [58] y [55]. Su principal objetivo es implementar el cálculo de los ICs de una manera rápida y sencilla. Actualmente cuenta con la implementación de doce ICs, donde es posible indicarle al programa cuáles de ellos calcular. Estos ICs son el LI ([7], [6]), el RLI ([73], [74]), el SALI ([78]), el MEGNO ([17], [16]) junto con el SElLCE ([17]), el FLI ([36], [50]), el OFLI ([32]), el APLE ([53]), los SSNs ([20], [86]), el DS ([87]), el GALI $2 \mathrm{~N}$ ([81]) y el OFLI $I_{\mathrm{TT}}^{2}([1])$.

La principal característica de este programa que lo convierte en una herramienta muy útil y versátil es la capacidad de calcular, para una o varias órbitas, el conjunto de ICs de una forma eficiente, seleccionando los ICs de acuerdo a las necesidades del estudio dinámico que se desea realizar. Esto quiere decir que el programa explota el hecho de que los distintos ICs comparten parte 
de su cálculo, fundamentalmente la resolución del conjunto de ecuaciones de movimiento y variacionales. Luego, el conjunto de ICs implementado ha sido dividido en unidades (las cuales serán descriptas en la Sección 3.6), agrupándolos siguiendo como criterio las operaciones que comparten. De esta manera, una vez que se haya indicado al programa los ICs a calcular, éste solamente llama a las rutinas correspondientes al cálculo de las unidades que contienen a dichos ICs. También calcula la evolución de cada vector desviación una única vez, independientemente de la cantidad de ICs que lo requieran para su cálculo. Esto logra una reducción en el tiempo de cómputo, y evita una redundancia en el cálculo.

\subsection{EL PROGRAMA PRINCIPAL}

El flujo del programa comienza con la lectura, desde un archivo de parámetros, de la información necesaria para el programa como, por ejemplo, los nombres de los archivos de entrada y salida, los ICs que se van a calcular, entre otros datos que detallaremos en la siguiente sección.

Luego de esto, el programa ingresa en un bucle que leerá, una a una, las condiciones iniciales de las órbitas a evolucionar. Para cada condición inicial que lea, el programa también lee los $2 \mathrm{~N}$ vectores desviación, donde $\mathrm{N}$ indica los grados de libertad del sistema.

Una vez que el programa leyó una condición inicial, ingresa en un bucle interior que se utilizará para avanzar el paso de la integración. Dentro de este bucle, el programa llama a la rutina que resuelve las ecuaciones diferenciales (o itera un paso de las ecuaciones del mapa, para el caso discreto). Para esto, comprueba qué unidades fueron seleccionadas para calcular, y configura la cantidad de vectores desviación que necesita resolver. El programa realiza una llamada a la rutina integradora por cada vector desviación que debe evolucionar, independientemente de cuántos ICs utilicen cada uno de estos vectores.

Para el caso de los ICs que necesitan renormalizar los vectores desviación, luego de la llamada a la rutina integradora, invoca una rutina que realiza dicha renormalización.

Una vez obtenidas las nuevas coordenadas, luego de efectuar un paso de integración, el programa llama, una a una, a las rutinas que calculan los ICs de cada unidad, de acuerdo a las que se hayan solicitado.

Luego de que estas rutinas devuelvan los valores de los ICs para las nuevas coordenadas, el programa realiza la escritura de los datos, guardando el valor de cada IC en un archivo distinto. Si se requirió el cálculo de la evolución de los ICs, la salida de los datos se escribirá cada cierto intervalo de tiempo. En cambio, si se solicitó la escritura de los valores finales, los archivos contendrán 
una sola línea, indicando las coordenadas de la condición inicial y el valor del IC correspondiente al tiempo $t \equiv t_{\text {final }}$.

\subsection{ENTRADA DE DATOS}

El programa comienza, como detallamos al inicio de la Sección 3.2, con la lectura de un archivo de parámetros. El contenido de este archivo es el siguiente:

- Nombre del archivo de entrada desde donde se leerán las coordenadas del espacio de fases y los vectores desviación que utilizará como condiciones iniciales.

- Los nombres de los archivos donde el programa guardará los resultados del cálculo de los indicadores. Estos parámetros definen un archivo para cada indicador.

- Un valor entero que actúa como puerta lógica (y lo llamaremos "valor entero lógico") para indicar si se guarda la evolución temporal de los ICs (asignando el valor 1) o si solamente se guarda su valor final (mediante la asignación del valor 0 ).

- Un valor numérico que le indica al programa, en el caso de que se escoja guardar la evolución de los ICs, el intervalo de tiempo de escritura de estos valores. En caso de que se haya escogido guardar sólo los valores finales, este parámetro es ignorado.

- Un nuevo valor entero lógico con el que se le informa al programa si, además de la evolución de los ICs, debe almacenar la evolución de las coordenadas del espacio de fases.

- Por último, quizá el conjunto de parámetros más importante, un arreglo de 6 valores enteros lógicos que le indican al programa sobre cuáles unidades (de las descriptas en la Sección 3.6) se deben calcular los ICs.

La otra parte importante de la entrada de datos se encuentra en un archivo (cuyo nombre es proporcionado en el archivo de parámetros) que contiene las condiciones iniciales de las órbitas que se desean integrar. En este archivo se proporciona, uno a uno, cada bloque de datos correspondiente a una condición inicial. Dicho bloque está compuesto por $2 \mathrm{~N}+1$ líneas. La primera línea contiene las coordenadas del espacio de fases de la condición inicial de la órbita, junto con el tiempo final de integración. Las $2 \mathrm{~N}$ líneas restantes proporcionan las coordenadas de los $2 \mathrm{~N}$ vectores desviación que definen a la partícula, independientemente de los ICs que se hayan seleccionado para calcular. De esta manera, existe la libertad de optar por introducir vectores desviación específicos, o bien introducir valores generados al azar. 


\subsection{EL INTEGRADOR}

Todos los indicadores hasta aquí implementados en el código deben integrar no sólo las ecuaciones de movimiento sino también las ecuaciones variacionales de primer orden y, en el caso del $\mathrm{OFLI}_{\mathrm{TT}}^{2}$, también se incluyen las de segundo orden. Por lo tanto, necesitamos contar con un integrador eficiente para esta tarea. En [24], hemos comprobado que una rutina de integración adecuada es la implementación de Prince y Dormand de un método Runge-Kutta de orden 7 - 8 llamado DOPRI8 ([71]). En ese trabajo mostramos que, para casos en los que debemos integrar las ecuaciones de movimiento y las variacionales de primer orden, DOPRI8 es más rápido que otros integradores, como por ejemplo uno basado en el método de Bulirsch-Stoer y otro basado en el método de Taylor.

Si bien existe un vasto número de integradores de ecuaciones diferenciales ordinarias (EDOs) en la literatura que pueden ser implementados en el LP-VIcode, la rutina DOPRI8 es el integrador de EDOs seleccionado para la actual versión.

La independencia de la rutina integradora con respecto al algoritmo integrador utilizado, forma parte de una implementación futura.

\subsection{CÁlculo de los ics}

Para el cálculo de los ICs a un dado tiempo t, el LP-VIcode llama a las diferentes rutinas de cálculo de acuerdo a las unidades que fueron "activadas" en el archivo de parámetros.

Los algoritmos encargados de calcular los ICs se han programado basándonos en las definiciones introducidas en las Secciones 2.2.1 a 2.2.8. Algunas de estas rutinas presentan ciertas particularidades en su implementación. Este es el caso de los ICs de la Unidad 3 (FLI, OFLI y APLE) y la Unidad $6\left(\mathrm{OFLI}_{\mathrm{TT}}^{2}\right)$. Si la condición inicial seleccionada corresponde a una órbita caótica, presentarán un crecimiento exponencial, alcanzando valores muy grandes en poco tiempo. Esto podría producir un desbordamiento en el código (es decir, que el valor de estos ICs exceden el límite para el almacenamiento de números de punto flotante). Para evitar este desbordamiento, el programa implementa un criterio de corte. Cuando alguno de estos ICs alcanza un valor lo suficientemente grande como para estar seguros de que la órbita en estudiada es caótica, el cálculo de éste se interrumpe, evitando el desbordamiento y, en consecuencia, reduciendo el tiempo de cómputo. El valor umbral que se ha adoptado en este trabajo para estos ICs es de $10^{20}$, ya que, en la mayoría de las simulaciones realizadas, el crecimiento del FLI y el OFLI para órbitas regulares es lineal con una pendiente del orden de la unidad. Por lo tanto, dado que los tiempos de integración considerados no exceden el orden de $\sim 10^{10}$ (o, como máximo, $10^{11}$ ), este valor 
de corte es suficiente para asegurarnos de que el comportamiento de la órbita no es regular.

Otros indicadores que presentan ciertas particularidades en su implementación son el $S A L I$ y el $G A L I_{2 \mathrm{~N}}$, que para órbitas caóticas su valor desciende hasta 0 . Debido a la precisión finita de la computadora se ha adoptado el valor $10^{-16}$ (que se corresponde con la precisión de ésta) como cero numérico. Esto es debido a que, como las magnitudes involucradas son del orden de la unidad (recordemos que, por ejemplo, el SALI se obtiene como suma o diferencia entre vectores unitarios), al realizar cálculos cuyos resultados sean magnitudes inferiores a dicha precisión, el resultado obtenido proviene de los errores de redondeo de la computadora.

\subsection{EL ORDENAMIENTO DE LOS ICS EN UNIDADES}

La mayoría de los ICs mencionados en el Capítulo 2 son completamente independientes entre sí y pueden ser calculados de forma separada. Las únicas excepciones a esto son el SElLCE ya que, al ser esencialmente la pendiente del $M E G N O$, depende estrictamente de los valores de éste para hacer el ajuste por mínimos cuadrados, y también el $R L I$, que se calcula como la diferencia entre los LIs de dos órbitas cercanas, haciendo que dependa de este último indicador.

Aunque sean indicadores cuyos cálculos son independientes, muchos de ellos comparten bloques de cálculo y es posible beneficiarnos de esas similitudes para poder calcular varios ICs juntos en una misma rutina y así disminuir el tiempo de CPU empleado. Tomemos como ejemplo el cálculo del FLI y del OFLI. Los algoritmos para obtenerlos son muy similares, empleando la evolución de los mismos $2 \mathrm{~N}$ vectores desviación (o de un solo vector desviación, dependiendo de qué variante del FLI se utilizará). Por este motivo sería conveniente, si necesitamos calcular ambos indicadores, utilizar la misma evolución de las ecuaciones, disminuyendo considerablemente el costo computacional. Por lo tanto, hemos decidido agrupar en unidades a los ICs que comparten parte de su proceso de cálculo. A continuación mostraremos la manera en que fueron agrupados, explicando el motivo por el cual comparten la unidad.

\subsubsection{Unidad 1}

LI: Dado que el ILCE y, por ende su implementación numérica, el LI, es el paradigma de los indicadores variacionales de caos, hemos decidido colocarlo en la primera unidad.

RLI: Como vimos en la Sección 2.2.2, éste se calcula a partir del cálculo de los LI de dos órbitas distintas muy próximas entre sí. Luego, dado que uno se 
esos dos $L I$ ya fue calculado, es conveniente ubicarlo en la misma unidad que este indicador, debiendo calcular solamente un LI adicional.

SALI: Este IC, como ya hemos descripto en la Sección 2.2.5, se calcula a partir de la definición de los índices paralelos y antiparalelos. Considerando que en este indicador lo que en realidad importa de los vectores desviación es su orientación, es necesario normalizarlos cada cierto intervalo de tiempo. Por lo tanto, dado que el $S A L I$ necesita de dos vectores desviación, y el $L I$ y el $R L I$ solamente uno, si colocamos al $S A L I$ en esta unidad, podría utilizar este vector y sólo necesitaríamos calcular la evolución de un vector desviación adicional. En cambio, si lo colocáramos en una unidad con un IC que necesite calcular los $2 \mathrm{~N}$ vectores desviación, el SALI demoraría más en su cómputo, ya que debe esperar a que se calculen $2 \mathrm{~N}-2$ vectores desviación que no necesita. Por otro lado, estos indicadores necesitan renormalizarse cada cierto intervalo de tiempo, por lo que, al estar juntos en la misma unidad, la renormalización se efectúa una única vez para todos los ICs, en el intervalo de tiempo correspondiente.

\subsubsection{Unidad 2}

MEgNo: Para un sistema de $\mathrm{N}$ grados de libertad, la mayoría de los ICs que utilizan la solución de las ecuaciones variacionales, necesitan $2 \mathrm{~N}$ ecuaciones para calcular las coordenadas en el espacio de las fases y, como máximo, $2 \mathrm{~N}$ ecuaciones para calcular la evolución de las ecuaciones variacionales de primer orden. El MEGNO, para llevar a cabo el cálculo de la cantidad $\bar{Y}$ (Sección 2.2.4), necesita incorporar dos ecuaciones diferenciales adicionales para resolver las Ecs. (2.25) y (2.26). Por ende, al necesitar de forma exclusiva la incorporación de estas dos ecuaciones adicionales, ha sido colocado en una nueva unidad.

SELLCE: La decisión de ubicarlo junto con el cáculo del MEGNO es trivial ya que el SElLCE, al ser la pendiente del MEGNO, necesita de su evolución temporal. Esta información se utiliza para construir la serie de puntos utilizada en el ajuste por mínimos cuadrados que estima dicha pendiente.

\subsubsection{Unidad 3}

FLI: La característica principal que define al FLI es la longitud de los vectores desviación, por lo que es necesario que no se normalicen dichos vectores durante la integración. Por otro lado, la definición introducida en [36] calcula el FLI a partir de la evolución de $2 \mathrm{~N}$ vectores desviación por lo que, si lo ubicamos en la primera unidad, los ICs que ya se encuentran allí verían su cómputo demorado, a causa de que se están calculando más vectores de los que necesitan, reducien- 
do su eficiencia. Como también hemos introducido la versión definida por [32], en la cual se utiliza sólamente un vector desviación, el FLI será implementado en esta nueva unidad.

OfLI: Como ya hemos visto en su definición, el OFLI es simplemente la componente ortogonal del FLI, necesitando de la evolución de los mismos vectores desviación que este último. Por ende, es evidente la conveniencia de ubicarlos en la misma unidad.

APLE: El caso del APLE es ligeramente distinto a estos dos últimos, ya que no es en sí un indicador nuevo, sino que es una manera de vincular el FLI con la entropía de Tsallis. Por ende, también utiliza $2 \mathrm{~N}$ vectores desviación sin normalizar. Esta última afirmación es suficiente para justificar su colocación en esta unidad.

\subsubsection{Unidad 4}

SSN: Los SSNs se calculan a partir de la construcción de histogramas, por lo que su cálculo es completamente distinto al de los ICs anteriores, haciendo necesaria la inclusión de esta nueva unidad.

Ds: Dado que el $D S$ se calcula a partir de las diferencias entre los histogramas anteriormente mencionados, es conveniente que sea agrupado junto con los SSNs.

\subsubsection{Unidad 5}

GALI$_{2 \mathrm{~N}}$ : Recordemos que, en realidad, el cálculo del $G A L I_{2 \mathrm{~N}}$ involucra el cómputo de varios ICs, más específicamente calcula $2 \mathrm{~N}-1$ GALIs. Por lo tanto utiliza desde 2 hasta $2 \mathrm{~N}$ vectores desviación para los distintos $G A L I_{\mathrm{k}}, \mathrm{y}$ además necesita de una subrutina para el cálculo de la descomposición en valores singulares (SVD). Como el método de cálculo es a partir de dicha subrutina, ha sido puesto en la unidad 5 .

\subsubsection{Unidad 6}

OFLI ${ }_{\mathrm{TT}}^{2}$ : Por último, la ubicación del OFLI $I_{\mathrm{TT}}^{2}$ en una nueva unidad es completamente trivial. Este IC es el único de nuestra lista de ICs que utiliza la solución de las ecuaciones variacionales de segundo orden, aumentando considerablemente el número de ecuaciones a integrar y, en consecuencia, el tiempo 
de cómputo. No obstante, como se ha mencionado en la Sección 2.2.7, este tiempo se puede reducir considerablemente.

Resumiendo, la forma en que han quedado agrupados los ICs en unidades dentro del LP-VIcode es como sigue:

- Unidad 1: LI, RLI y $S A L I$

- Unidad 2: MEGNO y SEILCE

- Unidad 3: FLI, OFLI y APLE

- Unidad 4: SSN y DS

- Unidad 5: GALI $2 \mathrm{~N}$

- Unidad 6: OFLI 2 TT

\subsection{TESTEO DEL PROGRAMA}

Hasta aquí se ha mencionado al LP-VIcode de una manera general, aplicable al estudio de cualquier sistema dinámico. De hecho existen dos versiones de este programa: una para el estudio de flujos hamiltonianos y otra para mapas simplécticos.

Las características que presentan estas dos versiones son muy similares. Una diferencia significativa que existe entre ambas es que, al ser la versión para mapas previa a la correspondiente para flujos hamiltonianos, el número de ICs implementados en la primera es menor. Los ICs que contiene son: el $L I$, el RLI, el SALI, el MEGNO, el FLI, los SSNs y el DS.

A continuación mostraremos algunos resultados de la aplicación de ambas variantes del LP-VIcode. La versión para mapas será aplicada a un sistema que ha sido utilizado en [74] y [78], entre otros. Consideremos un mapa simpléctico 4D que es una variante del mapa simpléctico 4D de Froeschlé ([33]) como nuestro sistema de prueba, definido por las siguiente ecuaciones

$$
\left\{\begin{array}{l}
x_{1}^{\prime}=x_{1}+x_{2} \\
x_{2}^{\prime}=x_{2}-v \operatorname{sen}\left(x_{1}+x_{2}\right)-\mu\left[1-\cos \left(x_{1}+x_{2}+x_{3}+x_{4}\right)\right] \\
x_{3}^{\prime}=x_{3}+x_{4} \\
x_{4}^{\prime}=x_{4}-\kappa \operatorname{sen}\left(x_{3}+x_{4}\right)-\mu\left[1-\cos \left(x_{1}+x_{2}+x_{3}+x_{4}\right)\right]
\end{array}\right.
$$

donde hemos escogido, al igual que los autores en [74] y [78], $v=0,5, \kappa=0,1$ y $\mu=10^{-3}$, y los $x_{i}$ son módulo $2 \pi$, de manera que $x_{i} \in[-\pi ; \pi), i=1, \ldots, 4$. 
Por otro lado, la versión para flujos hamiltonianos será aplicada al celebrado modelo introducido por Hénon y Heiles ([45]). Este modelo 2D puede describirse por medio del hamiltoniano

$$
H\left(x, y, p_{x}, p_{y}\right)=\frac{1}{2}\left(p_{x}^{2}+p_{y}^{2}\right)+\frac{1}{2}\left(x^{2}+y^{2}\right)+x^{2} y-\frac{1}{3} y^{3} .
$$

Se han escogido estos dos sistemas porque han sido ampliamente estudiados, y esto nos permite comparar algunos de nuestros resultados con los disponibles en la literatura, al efecto de mostrar su correcta implementación.

\subsubsection{Tiempos de CPU}

Presentaremos aquí los tiempos de cómputo empleados por el programa para calcular los ICs. Por un lado, mostraremos el tiempo promedio necesario para calcular cada uno de los ICs por separado. Por lo tanto, podremos contar con el tiempo total que emplean todos los indicadores correspondientes a una misma unidad (por ejemplo, el $L I$, el $R L I$ y el $S A L I$ ). Al resultado de la suma de estos tiempos lo compararemos luego con el tiempo que emplea el LP-VIcode en calcular cada unidad, a partir de la implementación que hemos realizado. Por último, compararemos la suma de los tiempos empleados por las 6 unidades contra el tiempo insumido por el cálculo combinado de éstas, es decir, colocando todos los valores enteros lógicos que indican el cálculo de una unidad en 1. Con esta última comparación intentamos mostrar las ventajas de compartir bloques de cálculo no sólo entre los ICs de una misma unidad, sino también entre las distintas unidades.

Para medir los tiempos de CPU para los programas antes mencionados, éstos fueron calculados con la siguiente configuración de hardware: CPU, 2 x Dual XEON 5450, Dual Core 3.0oGHz; M.B., Intel S500oVSA; RAM, 4GB(4X1GB), Kingston DDR-2, 667MHz, Dual Channel, con la versión 4.2.3 de gfortran, sin utilizar optimización.

Comenzaremos presentando los resultados para el modelo de Hénon y Heiles.

Para este sistema se han escogido tres órbitas regulares y dos órbitas caóticas, y las integramos durante un tiempo $t_{f}=2 \times 10^{4}$ u.t. (unidades de tiempo). Una escala de tiempo característico para este sistema es $T \sim 10$ u.t., por ende, el tiempo considerado para estos experimentos es del orden de $\sim 10^{3}$ tiempos característicos. Cabe aclarar que, para los indicadores de la unidad 3 (es decir, FLI, OFLI y APLE) hemos utilizado la variante que los calcula a partir de un único vector desviación. En la Tabla 3.1 se muestran los resultados obtenidos. 


\begin{tabular}{|c|c|c|c|c|c|}
\hline IC & $t_{C P U}$ & $t / t(L I)$ & \multirow[b]{4}{*}{ Unidad } & \multirow[b]{4}{*}{$t_{\mathrm{CPU}}$} & \multirow{4}{*}{$\mathrm{t}_{\mathrm{CPU}}^{\prime}$} \\
\hline$L I$ & 4,74 & 1,00 & & & \\
\hline$R L I$ & 8,45 & 1,78 & & & \\
\hline SALI & 5,35 & 1,13 & & & \\
\hline MEGNO & 6,37 & 1,34 & \multirow{4}{*}{$\begin{array}{l}1 \\
2 \\
3 \\
4\end{array}$} & \multirow{4}{*}{$\begin{array}{r}12,28 \\
20,80 \\
3,20 \\
39,99\end{array}$} & \multirow{4}{*}{$\begin{array}{r}18,54 \\
27,18 \\
9,69 \\
79,74\end{array}$} \\
\hline SEILCE & 20,81 & 4,39 & & & \\
\hline$F L I$ & 2,91 & 0,61 & & & \\
\hline OFLI & 3,02 & 0,64 & & & \\
\hline APLE & 3,76 & 0,79 & \multirow{5}{*}{ Total } & \multirow{5}{*}{120,44} & \multirow{5}{*}{179,32} \\
\hline SSNs & 39,53 & 8,34 & & & \\
\hline$D S$ & 40,21 & 8,48 & & & \\
\hline$G A L I_{2 \mathrm{~N}}$ & 23,95 & 5,05 & & & \\
\hline$O F L I_{\mathrm{TT}}^{2}$ & 20,22 & 4,27 & & & \\
\hline
\end{tabular}

Tabla 3.1: Tiempo de CPU (medido en segundos) para los distintos ICs (izquierda). La segunda columna muestra el tiempo empleado por cada IC. La tercera columna representa el $t_{C P U}$ normalizado al $t_{C P U}$ del LI. (Derecha) Tiempo de CPU para la unidad $i$, con $i=1,4$. La segunda columna indica el $t_{C P U}$ empleado por la unidad correspondiente, y en la tercera columna se muestra la suma de los tiempos empleados por todos los ICs que conforman dicha unidad.

En la tabla de la izquierda se muestra, en la segunda columna, el $t_{C P U}$, medido en segundos, empleado por cada IC de manera individual. Luego hemos representado, en la tercera columna, los $t_{\mathrm{CPU}}$ mostrados en la segunda columna normalizados con respecto al valor del $t_{C P U}$ del $L I$.

Podemos ver en esta tabla que los tiempos de CPU están estrechamente relacionados con la complejidad de los cálculos. Por ejemplo, el SElLCE debe realizar un ajuste por mínimos cuadrados, el $G A L I_{2 \mathrm{~N}}$, incluso utilizando la rutina SVD, no es eficiente en cuanto al tiempo de cómputo, ya que utiliza $2 \mathrm{~N}$ vectores desviación y además debe calcular $2 \mathrm{~N}-1$ indicadores, y por último, el OFLI $I_{\mathrm{TT}}^{2}$ utiliza la solución de las ecuaciones variacionales de segundo orden, que son muy costosas computacionalmente.

Por otro lado, existe un conjunto de ICs, los cuales mantuvieron sus tiempos de CPU (normalizados) en un rango de valores cercanos a la unidad. Los ICs que se han destacado en estos cálculos son los correspondientes a la unidad 3, especialmente el FLI. Esto se debe a que hemos implementado la versión de estos indicadores que utilizan solamente un vector desviación, al igual que el $L I$. Sin embargo, a diferencia de este último, los ICs de la unidad 3 implementan 
un corte en su cálculo cuando alcanzan un valor de saturación. Debido a esto, cuando estamos calculando los ICs en un espacio de fases dividido, con un porcentaje considerable de órbitas caóticas, para el FLI, el OFLI y el APLE, al igual que el $S A L I$ y el $G A L I_{2 N}$, los cálculos se detienen antes que otros indicadores debido al valor de saturación, y reducen el tiempo de CPU. Luego de realizar un estudio más exhaustivo de los tiempos de cómputo de los ICs, hemos encontrado que el FLI sigue siendo el IC más rápido. Por lo tanto, concluimos entonces que el FLI es el más apropiado para realizar cálculos de forma rápida $\mathrm{y}$, de hecho, es el que utilizaremos en la segunda parte de este trabajo para detectar las regiones de inestabilidad de nuestro sistema de estudio.

En el panel derecho de la Tabla 3.1 podemos comprobar la utilidad de la implementación realizada en el LP-VIcode. Allí se compara el tiempo que se emplea en el cálculo de una unidad contra la suma de los tiempos empleados en el cálculo de cada uno de los ICs que conforman dicha unidad de forma independiente. Por ejemplo, si medimos el tiempo total empleado en el cálculo de los ICs de la unidad 3 obtenemos un valor de $t_{C P U}=9,69$ segundos, donde cada uno de los tres ICs involucrados ha empleado aproximadamente el mismo tiempo. En cambio, si realizamos el cálculo de esta unidad con nuestra implementación del LP-VIcode, el valor obtenido para el tiempo de cómputo es de 3,20 segundos, es decir, muy cercano al tiempo insumido por un único IC.

En cuanto a la última fila de esta tabla, el primer valor muestra la suma del $t_{C P U}$ empleado en el cálculo de cada una de las 6 unidades de manera independiente. Por otro lado, en el segundo valor se refleja el $t_{C P U}$ empleado en el cómputo de todos los ICs, calculados uno por uno, sin la intervención de la implementación en unidades. Cabe aclarar que, dado que las unidades 5 y 6 poseen un solo indicador por unidad (a efectos de comparar los tiempos de CPU de las distintas unidades, hemos considerado a los $2 \mathrm{~N}-1 \mathrm{GALI}_{\mathrm{k}}$ como un mismo indicador), el $t_{\mathrm{CPU}}$ empleado por el IC y el correspondiente al calcular toda la unidad serán coincidentes. Por este motivo, no han sido incluidos en el panel derecho de la Tabla 3.1. Sin embargo, en los valores calculados en la última fila, correspondiente a las sumas de los tiempos, sí han sido tenidos en cuenta en ambos valores. Comparando estos dos resultados podemos destacar que la eficiente implementación del LP-VIcode logra reducir aproximadamente un $35 \%$ el tiempo de cómputo mediante el cálculo (por separado) de todas las unidades. A esta comparación debemos incorporar el tiempo empleado por el programa calculando todos los indicadores simultáneamente, activando el cómputo de todas las unidades en el archivo de parámetros. Mediante este cálculo obtuvimos un tiempo $t_{\text {total }} \approx 109,19$, que es considerablemente menor que los tiempos mostrados en la última fila del panel derecho de la Tabla 3.1. Esta reducción en el tiempo de cómputo es debido a que el LP-VIcode no solo aprovecha las operaciones en común que tienen los ICs de una misma unidad, 
sino que también tiene en cuentas las operaciones que tienen en común los ICs entre las distintas unidades.

Resulta evidente, entonces, la ventaja que representa la implementación del LP-VIcode para el cálculo de ICs.

\subsubsection{Aplicación a mapas simplécticos}

En esta sección presentaremos algunos resultados, producto del estudio de los sistemas definidos al comienzo de esta sección para mostrar que, además de disminuir el tiempo de cómputo en el cálculo de más de un IC de forma simultánea, éstos reproducen los resultados hallados por otros investigadores.

Los resultados de los cálculos que se muestran en esta sección fueron realizados sobre el mapa descripto en la Sección 3.7. Como simplemente queremos ilustrar la correcta implementación del LP-VIcode, consideramos dos condiciones iniciales, ya estudiadas en [74] y [78], entre otros, por lo tanto se encuentran bien identificadas. Una de ellas corresponde a una órbita regular $\left(x_{1}=0,5\right.$, $\left.x_{2}=0, x_{3}=0,5, x_{4}=0\right)$ y la otra corresponde a una órbita caótica $\left(x_{1}=3\right.$, $\left.x_{2}=0, x_{3}=0,5, x_{4}=0\right)$. Todos los indicadores están calculados hasta un número de iteraciones $\mathrm{N}_{i t e r}=10^{7}$. En la Fig. 3.I se presentan los gráficos correspondientes al cálculo del LI, del FLI, del DS, del SALI y del RLI. La curva con trazo rojo corresponden a la órbita caótica, mientras que la curva con trazo verde corresponde a la órbita regular.

El cálculo del $L I$ se muestra en el panel (a) de la Fig. 3.1. Se ve claramente que la órbita regular presenta un decrecimiento típico para este tipo de órbitas, es decir, del orden de $\ln \left(\mathrm{N}_{\text {iter }}\right) / \mathrm{N}_{\text {iter }}$, mientras que la órbita caótica se mantiene constante a partir de $\mathrm{N}_{\text {iter }} \sim 10^{4}$, en un valor $\sim 0,01$. Según el tipo de decrecimiento del LI ([79]) para una órbita regular, y considerando que en nuestro experimento tenemos $N_{\text {iter }}=10^{7}$, obtenemos que $\ln \left(N_{\text {iter }}\right) / N_{\text {iter }} \approx 1,61 \times 10^{-6}$. Por ende, vemos que el valor del $L I$ que obtuvimos para la órbita caótica es ampliamente superior al umbral mencionado, por lo que el resultado obtenido es consistente con la clase de órbita que es.

En la Fig. 3.I, panel (b), se muestra un gráfico de la evolución temporal del FLI. Aquí, la órbita regular sigue un crecimiento lineal $(F L I \propto t)$, mientras que la caótica crece exponencialmente hasta que satura para $N_{i t e r} \approx 4,2 \times 10^{3}$.

En el panel (c) presentamos la evolución de DS con respecto a $\mathrm{N}_{\text {iter }}$. Se puede ver un marcado decrecimiento lineal entre los logaritmos, indicando que los espectros se van asemejando cada vez más. Para la órbita regular la situación es opuesta: los espectros son bien distintos y nunca se asemejan entre sí, produciendo que la gráfica de $D S$ tienda a un valor constante (distinto de cero) en el tiempo. 

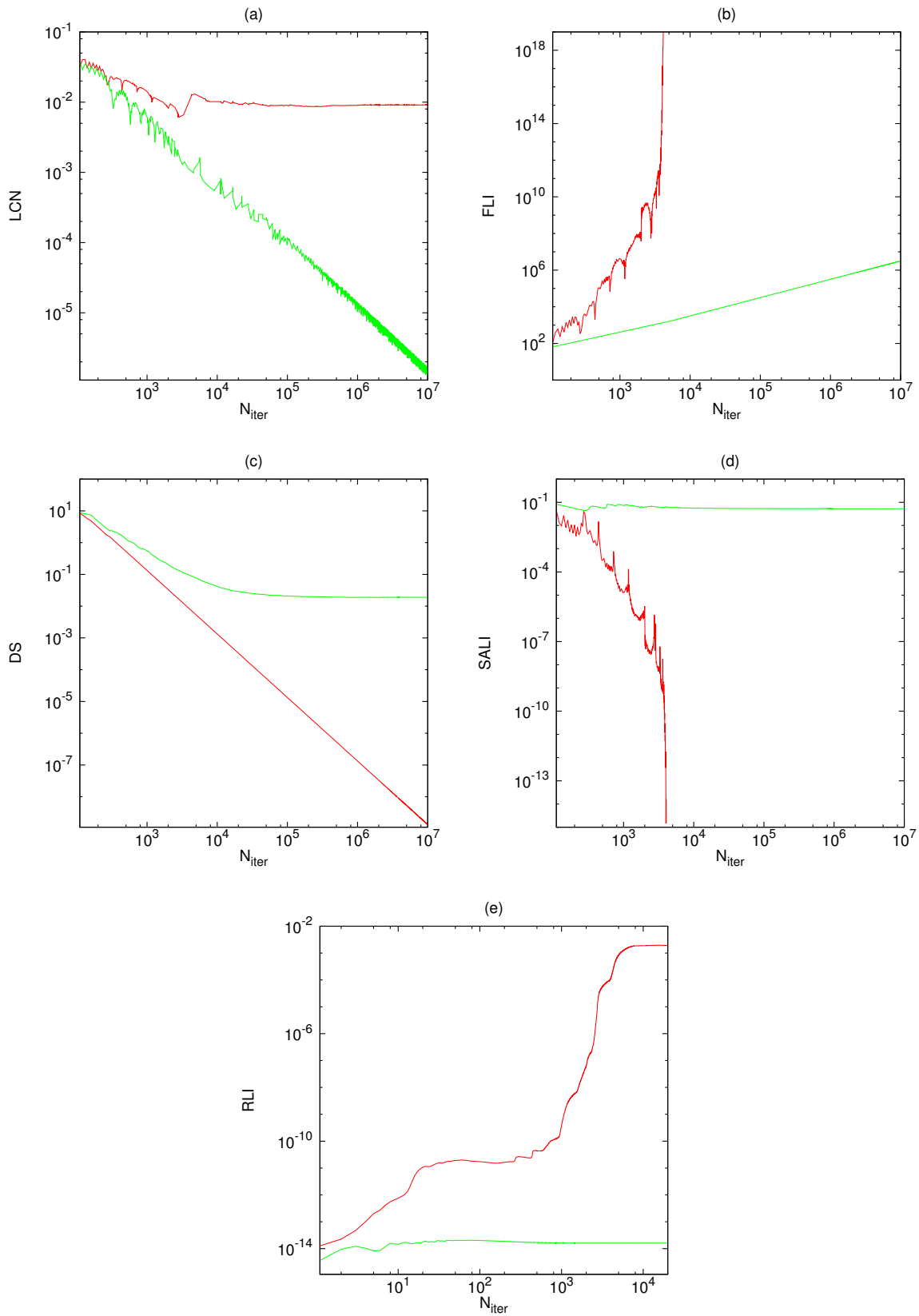

Figura 3.1: Gráficos de $L I(\mathrm{a}), F L I(\mathrm{~b})$, DS (c), $S A L I(\mathrm{~d})$ y $R L I(\mathrm{e})$, para una órbita regular $(0,5,0,2,0)$ y una órbita caótica $(3,0,1,0)$ en el rango de iteraciones $N_{i t e r} \in$ $\left(10^{2} ; 10^{7}\right)$ para los paneles (a) al (d), y en el rango $N_{i t e r} \in\left(0 ; 2 \times 10^{4}\right)$ para el panel (e). Se puede apreciar que el acuerdo de las cuatro primeras figuras con [78] es muy bueno, al igual que el panel (e) con [74]. 
El SALI, representado en el panel (d), presenta un comportamiento contrario al del FLI. Para la órbita caótica aquél decrece exponencialmente (la tendencia es muy similar a la del FLI, sólo que este último crece exponencialmente), saturando en nuestro valor de corte $10^{-16}$ considerado como "cero numérico". La órbita regular mantiene su valor constante, concordando con el hecho que los vectores desviación tienden a quedar fijos en direcciones distintas a lo largo del tiempo.

Por último, vemos en el panel (e) la evolución del $R L I$. Para este IC el cálculo se realizó hasta $\mathrm{N}_{\text {iter }}=2 \times 10^{4}$ iteraciones. Es claro cómo la órbita caótica tiene un crecimiento abrupto en el intervalo $\left[0 ; 5 \times 10^{3}\right]$ llegando a estabilizarse en un valor constante. La órbita regular, en cambio, permaneció constante en un valor $\sim 10^{-14}$. Esto es de esperar ya que, al tratarse de un indicador que mide la diferencia entre dos índices de Lyapunov, al ser una órbita regular, ambos índices tenderán a cero, al igual que su diferencia.

Todos los resultados aquí expuestos nos muestran que el LP-VIcode logra identificar correctamente tanto las órbitas caóticas como las regulares. Para complementar este análisis se pueden ver los paneles (a), (b), (c) y (d) de la Fig. 4 de [78] y compararlas con las correspondientes de nuestra Fig. 3.I. En esta comparación se puede verificar que los resultados que obtuvimos están en perfecto acuerdo con los obtenidos por Skokos et al. Por otro lado, de [74], se puede comparar la evolución del RLI entre el panel (b) de la Fig. 4 con nuestro correspondiente panel (e) de la Fig. 3.1. En esta comparación, a diferencia de las comparaciones anteriores, los valores obtenidos por Sándor et al. no guardan tal semenanza con nuestros resultados. Sin embargo, el hecho importante es que el comportamiento que siguen las evoluciones de las curvas del RLI es el mismo: por un lado, un crecimiento abrupto, seguido por una estabilización en un valor $\geqslant 10^{-4}$ para las órbitas caóticas $y$, por otro lado, para las órbitas regulares, la permanencia en un valor aproximadamente constante y relavitamente pequeño $\leqslant 10^{-12}$. Las diferencias observadas con [74] pueden deberse a que es posible escoger el valor de la separación inicial $\Delta \mathbf{x}_{0}$ en un amplio rango de valores, cubriendo el intervalo $10^{-14} \leqslant\left\|\Delta \mathbf{x}_{0}\right\| \leqslant 10^{-7}$.

Para el cálculo del MEGNO se utilizó el mismo mapa y las mismas condiciones iniciales que para el resto de los indicadores vistos más arriba. Dado que no contamos con cálculos del MEGNO realizados para este mapa, solamente analizaremos el comportamiento de las dos trayectorias. En el panel (a) de la Fig. 3.2 se puede ver el crecimiento del MEGNO correspondiente a una órbita caótica. Por otro lado, para la órbita regular (Fig. 3.2, panel (b)) vemos que la evolución de ésta sigue el comportamiento esperado, tendiendo a un valor constante determinado por la versión de $M E G N O$ que estemos utilizando. En este caso, al estar utilizando la versión $(2,0)$ del $M E G N O$, el valor asintótico al cual tiende es 0,5 . 

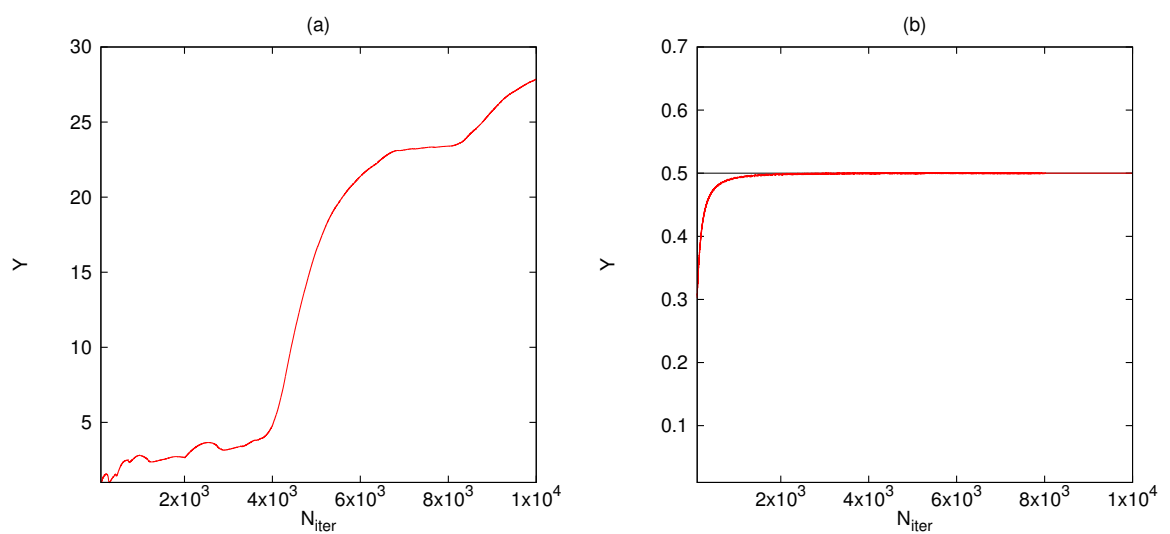

Figura 3.2: Evolución del MEGNO para una órbita caótica (a) con condición inicial $\left(x_{1}=3, x_{2}=0, x_{3}=0,5, x_{4}=0\right)$, y para una órbita regular $(b)$ con condición $\left(x_{1}=0,5, x_{2}=0, x_{3}=0,5, x_{4}=0\right)$. Ambos cálculos fueron hechos hasta un número máximo $\mathrm{N}_{\text {iter }}=10^{4}$ iteraciones.

\subsubsection{Aplicación a flujos hamiltonianos}

Continuando con los ensayos realizados sobre el LP-VIcode, vamos a mostrar en esta sección los resultados de la aplicación de la versión para flujos hamiltonianos. Para este propósito utilizamos el modelo de Hénon y Heiles (ver Sección 3.7), estudiado en numerosas publicaciones como, por ejemplo, [1], [32], [81], etc. Se escogió este sistema porque es bien conocido y con una extensa bibliografía que respalda los resultados publicados y facilita nuestra comparación.

\subsubsection{LI, SALI y $G A L I_{2 \mathrm{~N}}$}

Los resultados para el cálculo del $L I$, el $S A L I$ y los $G A L I_{2 \mathrm{~N}}$ se realizaron tomando como condición inicial la órbita caótica dada por $\left(x, y, p_{x}, p_{y}\right)=$ $(0 ;-0,25 ; 0,42 ; 0)$ y un valor $h=1 / 8=0,125$. Sus evoluciones se encuentran representadas en la Fig. 3.3.

En el panel (a) de la Fig. 3.3 mostramos la evolución del LI para la órbita mencionada, que sabemos a priori que ha sido identificada como caótica ([81]). Podemos ver que el $L I$ presenta el comportamiento correspondiente a una órbita caótica, esto es, tendiendo a estabilizarse en un valor constante, $\sigma \approx 0,042$.

En el panel (b) vemos el comportamiento del logaritmo de los $G A L I_{\mathrm{k}}$, con $k=2,3,4$, donde los tres siguen el decrecimiento exponencial esperado para una órbita caótica, siendo los GALIs de mayor orden los que decrecen más rápidamente (es decir, el $G A L I_{4}$ decrece más rápido que el $G A L I_{3}$ y éste, a su 

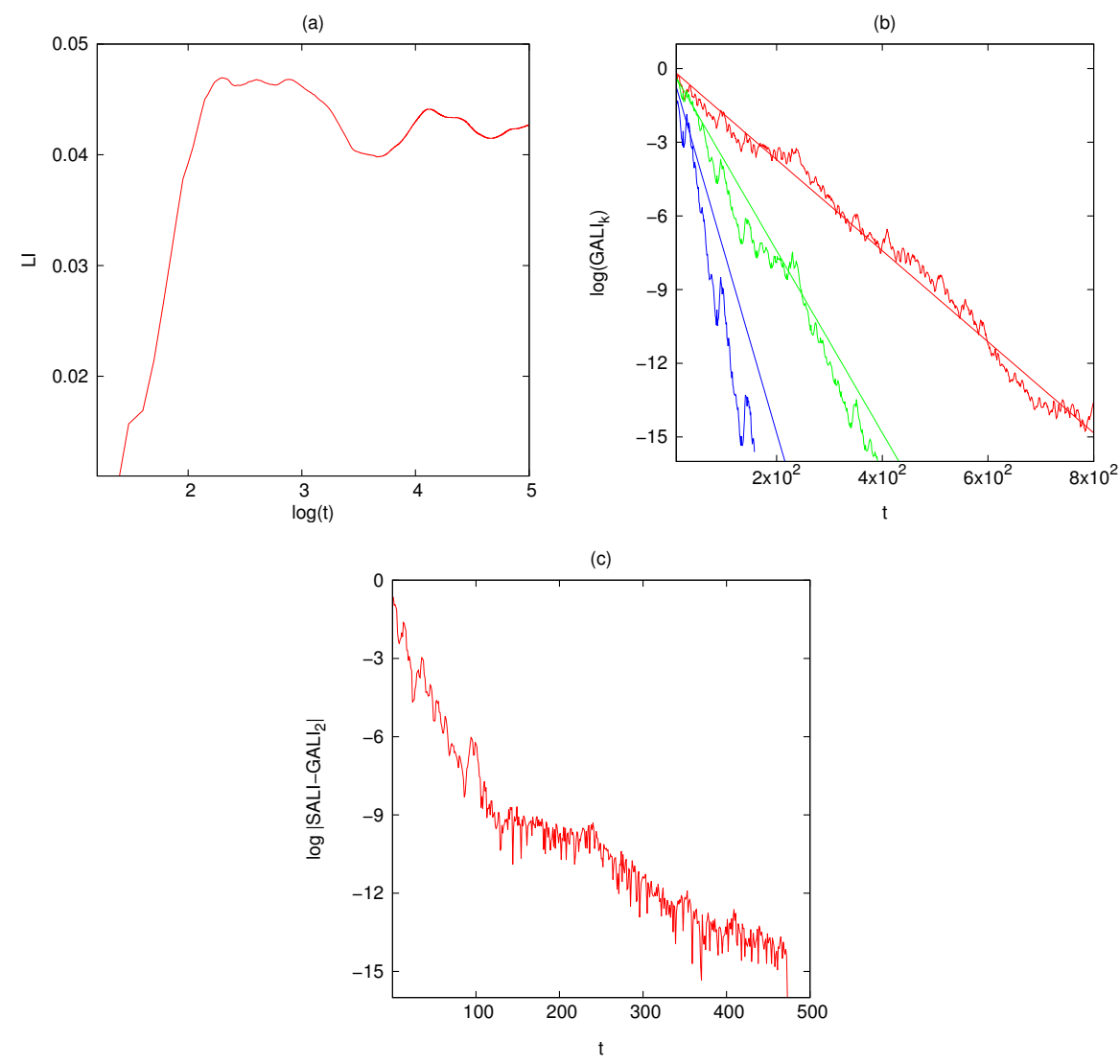

Figura 3.3: (a) Evolución del $L I$ con respecto a $\log (\mathrm{t})$, con $\log (\mathrm{t}) \in(1 ; 5)$ para una condición inicial $\left(x, y, p_{x}, p_{y}\right)=(0 ;-0,25 ; 0,42 ; 0)$, que se corresponde con un comportamiento caótico. (b) Evolución del $\log \left(\mathrm{GALI}_{\mathrm{k}}\right)$ con $\mathrm{k}=2$ (curva roja), $k=3$ (curva verde) y $k=4$ (curva azul) para la misma condición inicial. (c) Diferencia entre $S A L I$ y $G A L I_{2}$. Podemos ver que las dos últimas gráficas tienen una gran similitud con el panel (a) de la Fig. 1 y la Fig. 2 de [81]. 
vez, decrece más rápido que el $G A L I_{2}$ ). Este comportamiento se puede verificar del panel (b) de la Fig. I de [81].

Sabemos que, por definición, el $G A L I_{\mathrm{k}}$ coincide con el $S A L I$ para $\mathrm{k}=2$. Por ende, en el panel (c) hemos calculado la diferencia entre ambos ICs en un intento de cuantificar la proximidad numérica de ambas curvas en función del tiempo. Al igual que sucede en la Fig. 2 de [81], se logra llegar a la precisión de la computadora $\left(10^{-16}\right)$ en un tiempo considerablemente pequeño, aunque en el cálculo presentado en la Fig. 3.3 panel (c) le tomó un poco más de 450 u.t. para alcanzarlo.

Como es sabido, la tasa de decrecimiento del $G A L I_{\mathrm{k}}$ para órbitas caóticas es exponencial, con cierto exponente dado por el ILCE $\sigma_{1}$ ([8I]), ya que el inmediatamente inferior en nuestro caso es $\sigma_{2}=0$. Si los vectores desviación iniciales son elegidos al azar, esta dependencia será

$$
\mathrm{GALI}_{2} \propto e^{-\sigma_{1} \mathrm{t}}, \quad \mathrm{GALI}_{3} \propto e^{-2 \sigma_{1} \mathrm{t}}, \quad \mathrm{GALI}_{4} \propto e^{-4 \sigma_{1} \mathrm{t}} .
$$

En el panel (b) de la Fig. 3.3 han sido incluidas, junto con las curvas de los $\mathrm{GALI}_{k}$, las curvas correspondientes a las estimaciones teóricas representadas en la Ec. (3.2). Se puede ver que las evoluciones obtenidas numéricamente obedecen una relación muy semejante a esta estimación teórica.

Un fenómeno interesante para destacar es que, la cantidad de vectores desviación iniciales paralelos al flujo juegan un papel esencial en la velocidad con la que decrecen las curvas de los $\mathrm{GALI}_{k}$, con $k>2$ (ver [8I], Fig. 4). Por consiguiente, se realizaron cálculos para distintos números $m$ de vectores desviación iniciales paralelos al flujo $(\mathrm{m}=0,1$ y 2$)$. Estos cálculos fueron realizados para una órbita regular, con condiciones iniciales $\left(x, y, p_{x}, p_{y}\right)=(0,0,1 / 2,0)$. Los vectores desviación tomados en cada caso son

\begin{tabular}{|l|l|l|}
\hline $\mathrm{m}=0$ & $\mathrm{~m}=1$ & $\mathrm{~m}=2$ \\
\hline$(1,0,0,0)$ & $(1,0,0,0)$ & $(1 / 2,1 / 2,-1 / 2,-1 / 2)$ \\
$(0,0,0,1)$ & $(1 / 2,1 / 2,1 / 2,1 / 2)$ & $(1 / 2,1 / 2,1 / 2,1 / 2)$ \\
$(0,0,1,0)$ & $(0,0,1,0)$ & $(0,0,1,0)$ \\
$(0,1,0,0)$ & $(0,1,0,0)$ & $(0,1,0,0)$ \\
\hline
\end{tabular}

En el panel (a) de la Fig. 3.4 presentamos la evolución del $G A L I_{3}$ para los tres valores distintos de $m$, mientras que en el panel (b) presentamos el cálculo análogo para el $\mathrm{GALI}_{4}$. La correspondencia de cada curva con la pendiente de su ajuste es notable. Estas pendientes fueron calculadas utilizando un ajuste por mínimos cuadrados, obteniendo una diferencia con los valores teóricos $<1 \%$. Los valores teóricos $\alpha$ de las pendientes para el $G A L I_{3}$ son $\alpha=-2$ para $m=0$, 

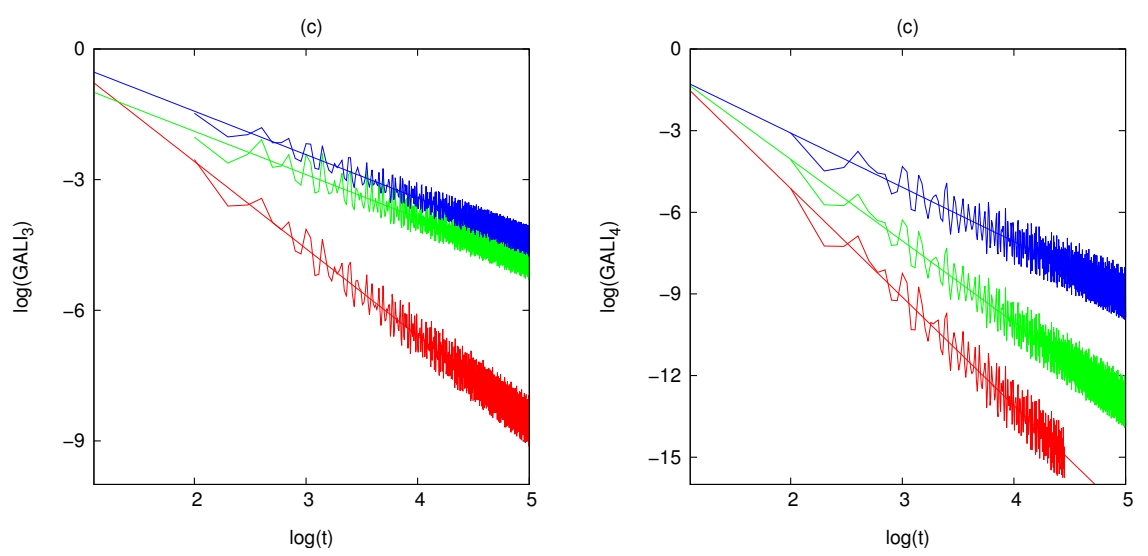

Figura 3.4: Evolución temporal del $\mathrm{GALI}_{3}$ (a) y $\mathrm{GALI}_{4}$ (b) para una órbita regular con $x=0, y=0, p_{x}=1 / 2$ y $p_{y}=0$, para distintos números $m$ de vectores desviación iniciales paralelos al flujo $(m=0,1,2)$, junto con sus estimaciones lineales. Las curvas de trazo rojo corresponden a $m=0$, las de trazo verde a $m=1$ y las de trazo azul a $m=2$.

y $\alpha=-1$ tanto para $\mathrm{m}=1$ como para $\mathrm{m}=2$, lo cual es posible verlo en la gráfica. Para el $\mathrm{GALI}_{4}$, los valores de $\alpha$ son $\alpha=-4,-3,-2$ para $\mathrm{m}=0,1 \mathrm{y}$ 2 , respectivamente. Este acuerdo entre los valores teóricos y sus estimaciones numéricas nos proporciona un fundamento adicional sobre el cual apoyarnos para confiar en la implementación del $G A L I_{2 \mathrm{~N}}$ realizada en el LP-VIcode.

\subsubsection{FLI, OFLI y APLE}

Para el cálculo de estos ICs se utilizaron tres condiciones iniciales considerando un valor $h=1 / 12 \approx 0,0833$ ([32]): una correspondiente a una órbita caótica, con coordenadas $(y, \dot{y})=(-0,121 ; 0)$, una órbita regular no periódica con $(y, \dot{y})=(0,2 ; 0)$ y una órbita regular periódica con condiciones iniciales $(y, \dot{y})=(0,25480176 ; 0)$. En los tres casos se ha considerado $x=0 y \dot{x}$ se obtiene a partir de la resolución de la Ec. (3.1) para el valor de la energía escogido.

En el panel (a) de la Fig. 3.5 podemos ver la evolución temporal del $\log (F L I)$. La órbita caótica crece rápidamente, saturando para $t \approx 500$. Dado que $t_{f}=10^{4}$, se ha considerado que un valor de saturación para este cálculo de $10^{10}$ sería suficiente para lograr discriminar un comportamiento regular de uno caótico. Por otro lado, ambas órbitas regulares presentan un crecimiento lineal. Si comparamos este gráfico con la Fig. 5 de [32] podemos apreciar que el comportamiento de la órbita caótica es muy similar. Por otro lado, para las órbitas regulares, si bien no tienden exactamente al mismo valor final en ambos gráficos, presentan una gran similitud en el tipo de comportamiento del crecimiento, que es el correspondiente a una órbita regular. En el panel (b) realizamos el mismo cálcu- 

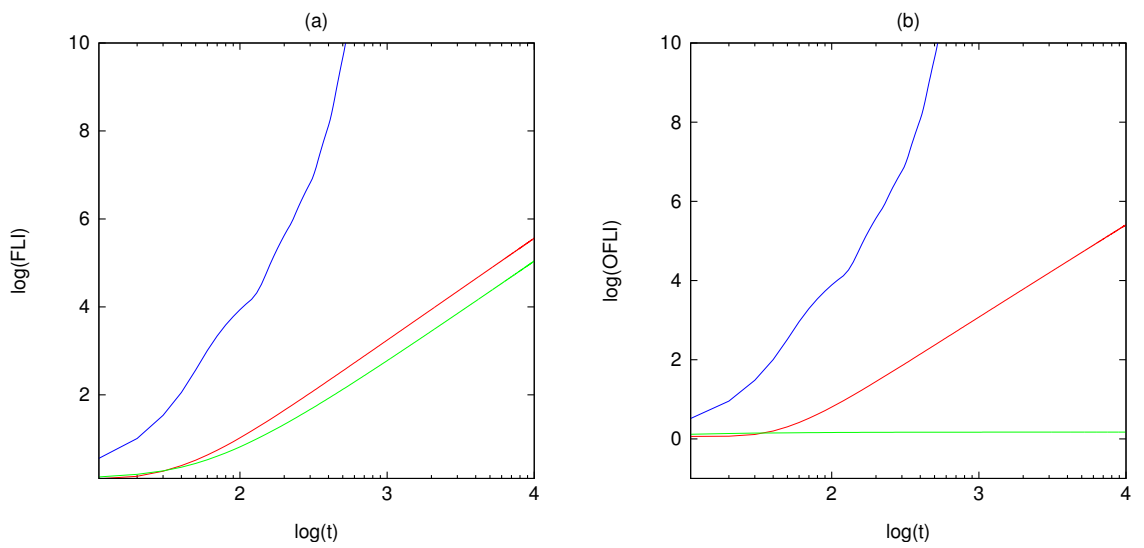

Figura 3.5: Evolución temporal del FLI (a) y del OFLI (b) para el potencial de Hénon y Heiles con $\mathrm{H} \approx 0,0833$ para tres órbitas distintas: una órbita caótica (representada por la línea azul) con $(y, \dot{y})=(-0,121 ; 0)$, una órbita regular no periódica (representada por la línea roja) con $(y, \dot{y})=(0,2 ; 0)$ y una órbita regular periódica (representada por la línea verde) con $(y, \dot{y})=0,25480176 ; 0)$.

lo que en el panel (a) pero para la estimación del OFLI. Los comportamientos correspondientes a la órbita caótica y a la regular no periódica (curvas azul y roja, respectivamente) no presentan variaciones significativas. Con respecto a la órbita pediódica, ésta presenta un cambio en su comportamiento, el cual manifiesta un ligero crecimiento al comienzo de la integración y luego permanece aproximadamente constante. Comparando con la Fig. 7 de [32] podemos ver nuevamente que ambos gráficos son consistentes entre sí. Esto permite concluir que el FLI y el OFLI fueron implementados de forma correcta.

El otro indicador que también forma parte de la unidad 3 es el APLE. Podemos ver en la Fig. 3.6 su evolución temporal para las mismas tres órbitas que para el FLI y el OFLI. Puede verse en el gráfico cómo la órbita caótica crece exponencialmente, mientras que las órbitas regulares muestran una tendencia asintótica hacia el valor 1 , lo que indicaría que el crecimiento de $w(t)$ se acerca a un comportamiento lineal.

\subsubsection{MEGNO y SEILCE}

Para el cálculo de estos dos indicadores vamos a utilizar la energía $h=0,118$, para poder realizar la comparación con los resultados publicados en [17]. Los análisis se llevaron a cabo para 5 órbitas sobre la superficie $x=0$. En la Fig. 3.7, panel (a) mostramos el cálculo del MEGNO para una órbita estable periódica con condiciones iniciales $\left(y, p_{y}\right)=(0,295456 ; 0)$ (simbolizada con $\left.s p\right)$, una cuasiperiódica $\left(y, p_{y}\right)=(0,483 ; 0)(q p)$ y una también cuasi-periódica pero cercana a una 4 -periódica inestable, con $\left(y, p_{y}\right)=(0,46912 ; 0)$ (up). Hemos graficado 


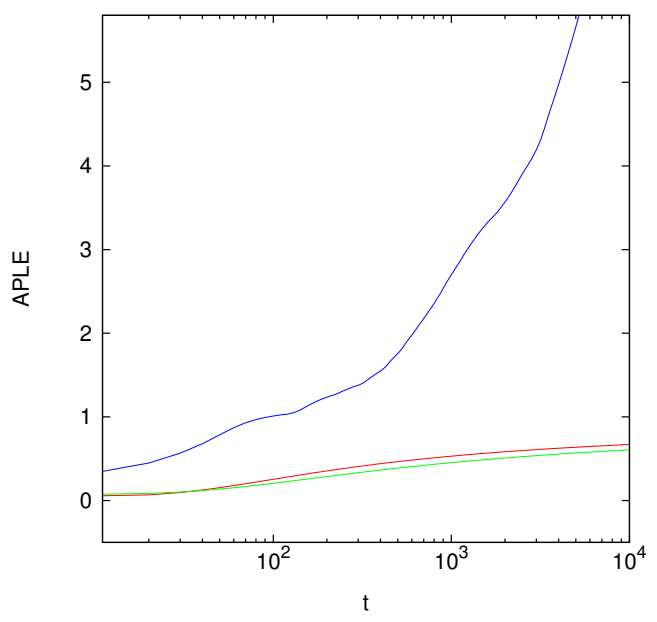

Figura 3.6: Evolución temporal del APLE para el potencial de Hénon \& Heiles, para tres órbitas distintas: una órbita caótica $(y, \dot{y})=(-0,121,0)$, una órbita regular resonante $(y, \dot{y})=(0,2,0)$ y una órbita periódica $(y, \dot{y})=(0,25480176,0)$.

también la recta $y=2$, que corresponde al umbral teórico del MEGNO para órbitas regulares (recordemos de la Sección 2.2.4.I que, para flujos hamiltonianos, utilizamos la variante $(1,-1)$ del MEGNO). Vemos en la figura que, tanto la órbita $s p$ como la $q p$, tienden al valor teórico, pero por valores menores que éste, por tratarse de órbitas estables ([17]). En cambio, para la up, si bien el tiempo de integración no ha sido suficiente para que su tendencia al valor 2 sea evidente, se puede observar que tiene un comportamiento decreciente y, estudios con realizados para tiempos de integración mayores (que no serán mostrados en este trabajo), han mostrado una clara tendencia hacia este valor. Esta tendencia, sin embargo, a diferencia de las evoluciones de $s p$ y $q p$, la lleva a cabo por encima del valor límite 2. Este comportamiento es el correspondiente a una órbita cuasiperiódica, muy próxima a una periódica inestable, y es idéntico al mostrado en [17], en el panel (d) de la Fig. 1.

Para el caso de órbitas caóticas, en la Fig. 3.7, panel (b) utilizamos, también sobre la superficie $x=0$, las siguientes condiciones iniciales: $\left(y, p_{y}\right)=(0,509 ; 0)$ (c1) y $\left(y, p_{y}\right)=(0,56 ; 0,112)(c 2)$. Su evolución presenta un crecimiento claramente lineal (en escala logaritmica), al igual que podemos ver en la Fig. 1, panel (c) de [17].

En el panel (c) de la Fig. 3.7 podemos ver la evolución temporal del SElLCE, para las 5 órbitas simultáneamente. Vemos que para las órbitas caóticas (c1) y (c2), desde $t \sim 6 \times 10^{3}$, presenta un comportamiento aproximadamente constante, debido al crecimiento lineal que tiene el MEGNO para este tipo de órbitas (ver panel (b)). Por el contrario, para las órbitas regulares $(s p),(q p)$ y (up), como el MEGNO tiende al valor de umbral teórico 2, es lógico que su pendiente 

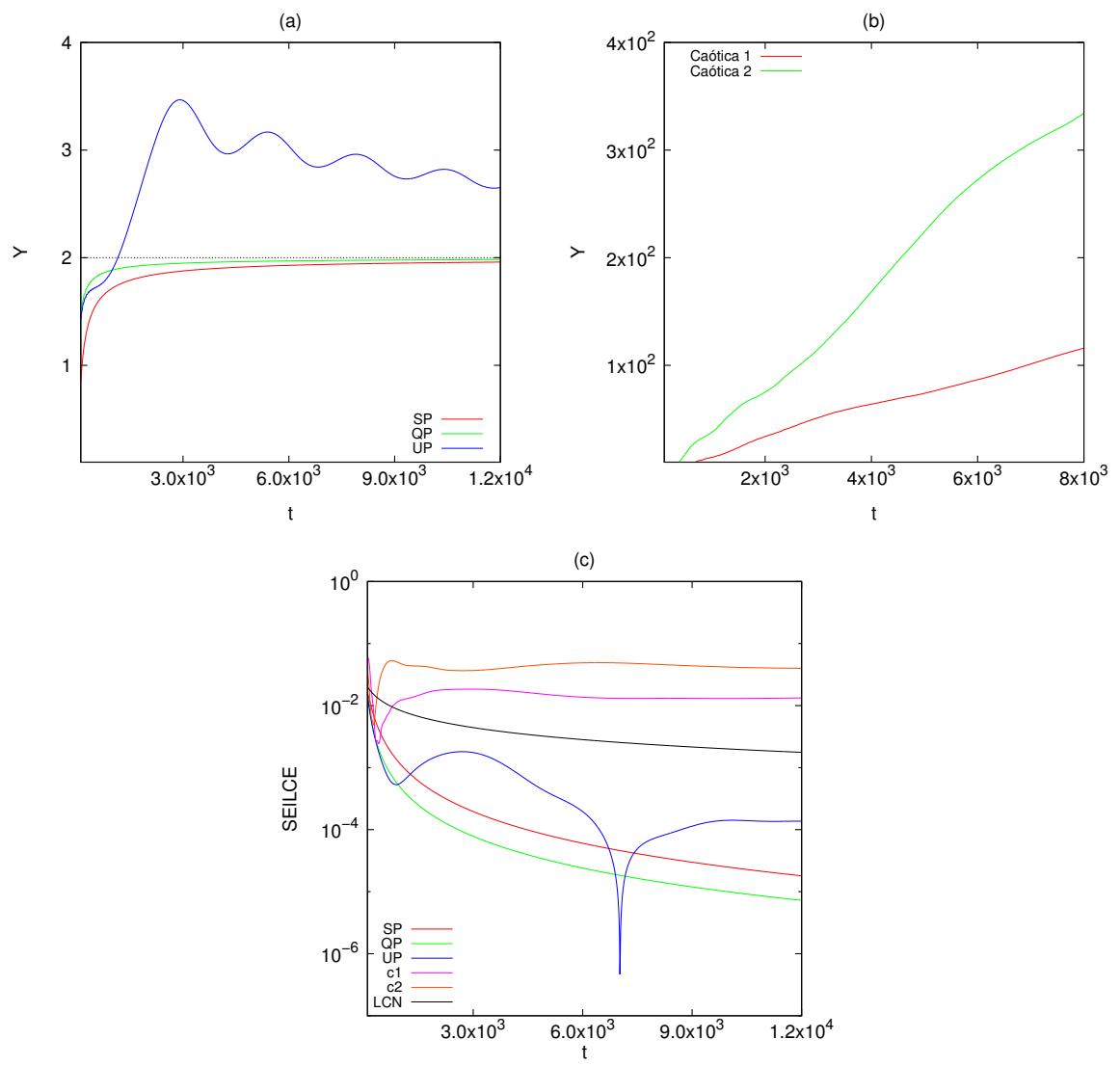

Figura 3.7: Evolución temporal del MEGNO, con $t \in\left(0 ; 1,2 \times 10^{4}\right)$ para tres órbitas regulares (a): Una periódica estable $(s p)$ con $\left(x_{0}, y_{0}, p_{x_{0}}, p_{y_{0}}\right)=(0 ; 0,295456 ; 0,407308 ; 0)$, una cuasi-periódica estable $(q p)\left(x_{0}, y_{0}, p_{x_{0}}, p_{y_{0}}\right)=(0 ; 0,483 ; 0,27898 ; 0)$ y una cuasi-periódica inestable (up) $\left(x_{0}, y_{0}, p_{x_{0}}, p_{y_{0}}\right)=(0 ; 0,46912 ; 0,291125 ; 0)$. (b) Evolución del MEGNO para 2 órbitas caóticas: $(c 1)\left(x_{0}, y_{0}, p_{x_{0}}, p_{y_{0}}\right)=(0 ; 0,509 ; 0,254625 ; 0)$ y $(c 2)$ $\left(x_{0}, y_{0}, p_{x_{0}}, p_{y_{0}}\right)=(0 ; 0,56 ; 0,164114 ; 0,112)$. (c) Evolución temporal del SEILCE para las órbitas de los paneles (a) y (b). 
tienda a o, ya que las evoluciones tienden a ser cada vez más lentas. Junto con la evolución de las 5 condiciones iniciales se incorporó en la figura, con trazo negro, la evolución del $L I$ para la órbita $(s p)$, la cual se ve que decrece más lentamente que el SElLCE, conclusión ya destacada en [17].

\subsubsection{RLI, DS y SSN}

Al igual que en la Sección 3.7.3.1, los experimentos realizados en esta sección fueron llevados a cabo para $\mathrm{H}=0,125$, y utilizando las mismas condiciones iniciales.

En la Fig. 3.8 mostramos la evolución del RLI con respecto a t. Podemos ver que, para la órbita regular (línea verde), su valor es aproximadamente constante en toda la integración $\left(\sim 5 \times 10^{-12}\right)$. Por otro lado, para la órbita caótica (curva roja), vemos que en el intervalo $t \in\left(0 ; 7,5 \times 10^{2}\right)$ el $R L I$ crece hasta un valor aproximadamente constante $0,01 \leqslant \log (R L I) \leqslant 0,1$. Ambas evoluciones obedecen el comportamiento correspondiente a una órbita regular y a una caótica, respectivamente.

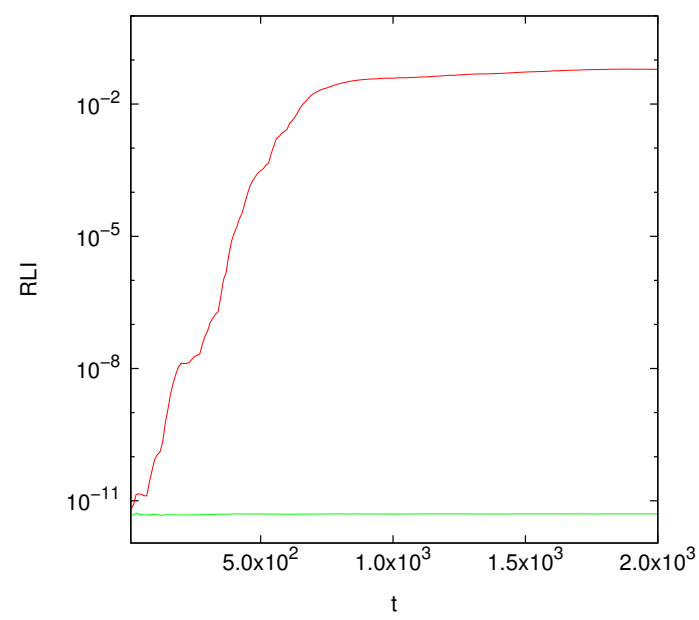

Figura 3.8: Evolución temporal del RLI para una órbita caótica con condiciones iniciales $(0 ;-0,25 ; 0,42 ; 0)$ (línea roja), y una órbita regular con condiciones iniciales $(0 ; 0 ; 0,5 ; 0)$ (línea verde).

En cuanto al SSN, podemos ver en el panel (a) de la Fig. 3.9 el gráfico correspondiente a los espectros de la órbita regular. Como ya habíamos mencionado en la Sección 2.2.3, debido a que para una órbita regular sus vectores desviación se mantienen fijos en una dada dirección a lo largo del tiempo, dicha órbita tendrá espectros bien diferentes, como observamos en este panel. Asimismo, para una órbita caótica, los vectores desviación tienden a coincidir en dirección, al igual que lo harán sus espectros. En el panel (b) se presentan los espectros correspondientes a la órbita caótica. Se evidencia aquí que los SSNs siguen el 
comportamiento descripto para este tipo de órbitas, produciendo que la curva roja quede oculta tras la verde, debido a que estas dos curvas coinciden espacialmente.

Este comportamiento descripto puede comprobarse calculando el DS (Fig. 3.9, panel (c)). Dado que, para una órbita regular, sus espectros son notablemente diferentes, la distancia entre éstos permanece constante en un valor distinto de 0. Esto está representado en la figura con la línea de trazo verde. Por otro lado, para las órbitas caóticas, como los espectros tienden a coincidir, su distancia disminuirá con el tiempo, produciendo la curva decreciente, representada con trazo rojo, que se muestra en este panel.
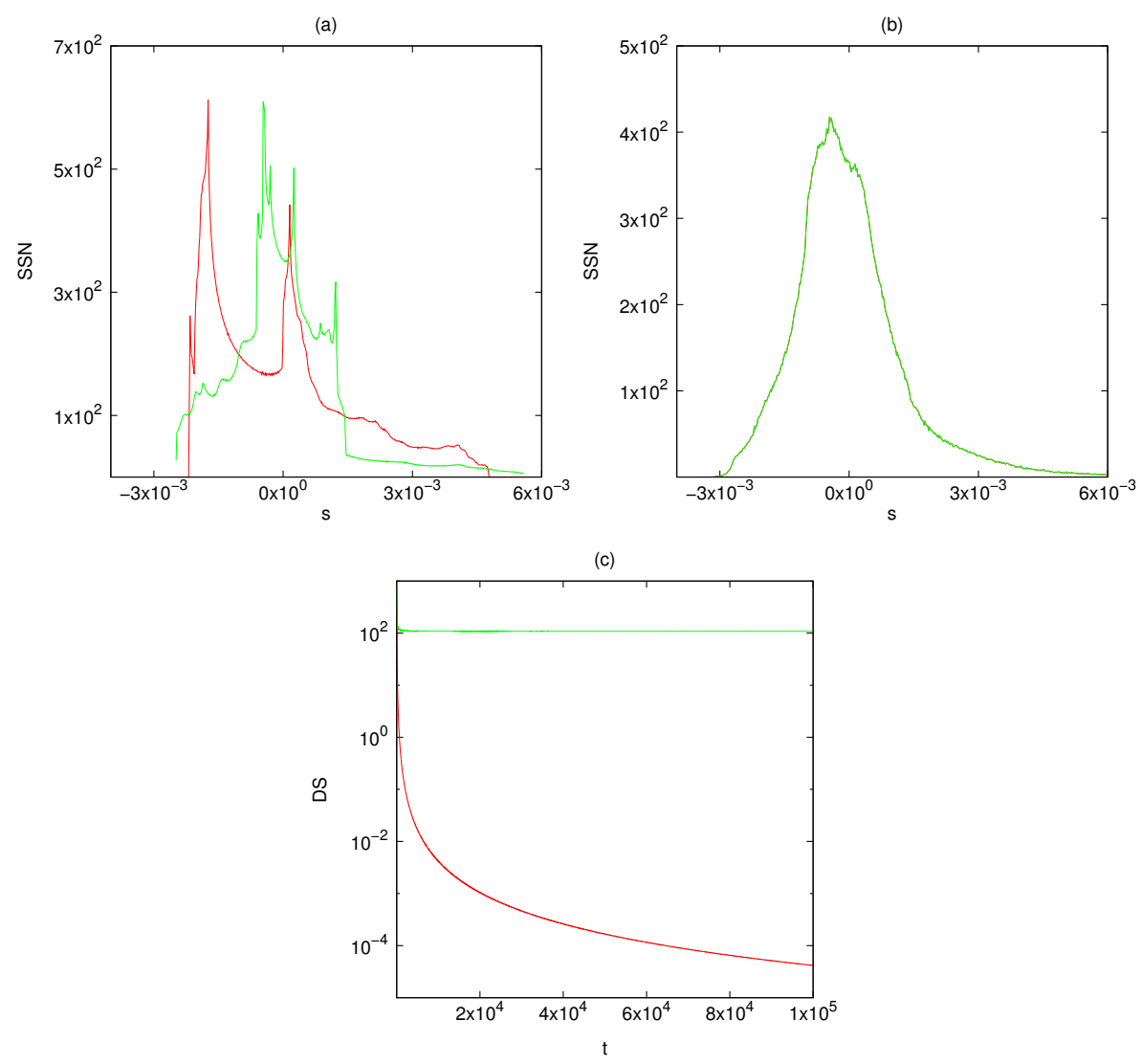

Figura 3.9: Gráfico del $S S N$ para una órbita regular con condición inicial $\left(x, y, p_{x}, p_{y}\right)=$ $(0 ; 0 ; 1 / 2 ; 0)(a)$, y una órbita caótica con condición inicial $\left(x, y, p_{x}, p_{y}\right)=$ $(0 ;-0,25 ; 0,42 ; 0)($ b). (c) Evolución de DS con respecto a t para las dos condiciones iniciales de los paneles (a) (con trazo verde) y (b) (con trazo rojo). 


\subsubsection{OFLI TT}

Por último, se mostrarán algunos resultados del cálculo del OFLI TT. Para ello hemos reproducido el gráfico correspondiente al panel medio de la Fig. 3 de [1]. En el panel (a) de la Fig. 3.10 vemos representado el valor final del OFLI $I_{\mathrm{TT}}^{2}$ para un conjunto de condiciones iniciales. Se ve que algunos de estos valores han alcanzado el umbral de saturación $\ln \left(10^{20}\right) \approx 46$. Estas condiciones iniciales fueron construidas sobre el plano $x=p_{y}=0$ tomando $1,1 \times 10^{3}$ valores en el rango $-0,4 \leqslant y \leqslant 0,7$, con $h=1 / 8$.
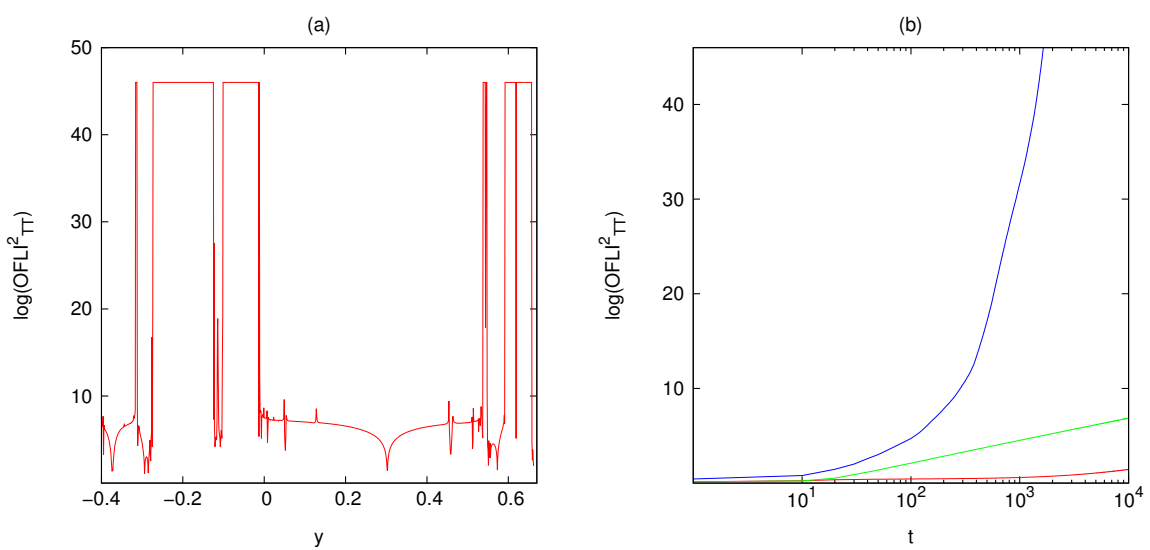

Figura 3.10: a) Valores del OFLI $I_{\mathrm{TT}}^{2}$ para un conjunto de condiciones iniciales en el plano $x=p_{y}=0$ con valores de $y-0,4 \leqslant y \leqslant 0,65 y h=1 / 8$. (b) Evolución temporal del OFLI TT para tres órbitas: una regular correspondiente al mínimo del IC en $y=0,303$, otra regular para un valor intermedio del OFLI TT en $y=0,15$ y una órbita caótica en $y=-0,15$. Todos los cálculos fueron realizados para $t_{f}=10^{4}$.

Para la construcción del panel (b) hemos calculado la evolución temporal del $O F L I_{\mathrm{TT}}^{2}$ para tres órbitas distintas: una órbita regular, que se corresponde con el mínimo del $O F L I_{\mathrm{TT}}^{2}$ con condición inicial y $=0,303$, otra órbita regular, para un valor intermedio del indicador, en $y=0,15$ y una órbita caótica en $y=-0,15$. Observando las curvas obtenidas, vemos que ambas órbitas regulares crecen linealmente, en escala logarítmica, con una pendiente muy baja. Si nos focalizamos en la evolución correspondiente a la órbita caótica obtenemos claramente, también en escala logarítmica, un crecimiento exponencial. Los valores finales de estas evoluciones temporales coinciden con los valores reflejados en el panel (a) para estas tres condiciones iniciales. 


\subsection{DISCUSIÓN}

En este capítulo se ha presentado un código, escrito en Fortran 77, que implementa de forma eficiente un conjunto de doce indicadores variacionales de caos. Estos ICs son: el LI, el RLI, el SALI, el MEGNO, el SElLCE, el FLI, el OFLI, el APLE, los SSNs, el DS, los GALI $2 \mathrm{~N}$ y el OFLI TT

En las distintas secciones hemos descripto cómo está construido el programa principal, cómo opera la entrada de datos, el integrador utilizado (en el caso del cálculo en flujos hamiltonianos) y cómo están agrupados los ICs en unidades, de acuerdo a las operaciones que éstos comparten.

Una vez introducido el código se han presentado resultados realizados con las dos variantes existentes.

Hemos medido los tiempos de CPU empleados por cada IC de manera individual para la variante de flujos hamiltonianos, en el caso del modelo de Hénon y Heiles. Luego medimos los tiempos correspondientes para cada unidad, y los comparamos con la suma de los tiempos de cada indicador perteneciente a esa unidad. Los resultados arrojaron que esta agrupación en unidades mejora el tiempo de cómputo en aproximadamente un $35 \%$. También se midió el tiempo empleado por todo el programa completo, activando el cálculo de todos los ICs, y se lo comparó con la suma de los tiempos de cada unidad. Con esto mostramos que el tiempo empleado por todo el programa es aproximadamente un $10 \%$ menor que la suma de los tiempos empleados por las unidades. Estos resultados nos muestran que el código fue implementado de manera eficiente, reduciendo considerablemente los tiempos de CPU.

Luego de esto procedimos a mostrar las evoluciones temporales de los ICs para ilustrar la correcta implementación del LP-VIcode, llevando a cabo los cálculos para ambas versiones. Se ha probado que el código, además de estar implementado de forma eficiente en cuanto a los tiempos de cómputo, reproduce correctamente el comportamiento tanto de órbitas caóticas como regulares.

Por lo tanto concluimos que hemos podido construir una herramienta que nos permite calcular de forma eficiente un extenso conjunto de ICs, permitiendo una gran personalización de éste, desde la elección de cuáles ICs queremos calcular hasta poder elegir calcular la evolución de éstos o sólo su valor final. 

Parte II

CUANTIFICACIÓN DE LA DIFUSIÓN 



\section{4}

ESTUDIO DE LA DIFUSIÓN MEDIANTE EL CÁLCULO DE FORMAS NORMALES

Supóngase un sistema conservativo, sea éste un flujo hamiltoniano (como en [18], [29], [42], [51]) o un mapa simpléctico ([38], [39], [75], [85]). Si el sistema fuese integrable, las acciones serían integrales de movimiento, por lo tanto permanecerían constantes, y una órbita quedaría representada, en el espacio de las acciones, por un punto. En cambio, si dicho sistema presenta un espacio de fases dividido, estas acciones varían en el tiempo, ya sea oscilando alrededor de un valor central correspondiente a la acción sin perturbar, o presentando una variación secular, fenómeno conocido como difusión. La difusión puede ser definida de una forma sencilla como la variación secular de las integrales no perturbadas, definición utilizada por [12], [13], [40], [59], [62], entre otros.

El estudio de la difusión es clave para comprender la dinámica de un sistema, ya que permite analizar cómo varían las acciones no perturbadas en el tiempo que pueden modificar su estructura. Consideremos un sistema galáctico. En este sistema, las acciones o integrales representan magnitudes que funciones de la energía y el momento angular de la órbita de una estrella. Si las acciones presentan una variación secular, la forma de las órbitas cambiará en el tiempo. Otro ejemplo es el de un planeta orbitando en torno a su estrella (problema de 2 cuerpos), en cuyo caso las acciones son funciones de los elementos orbitales. Pensemos en el semieje a y la excentricidad e. En este caso, el sistema es integrable, entonces tanto a como e son constantes, y el planeta se mueve en una órbita regular. Pero, si se tiene en cuenta la presencia de otros cuerpos celestes, como ocurre con los gigantes gaseosos en nuestro Sistema Solar, el sistema se ve perturbado y los elementos orbitales del cuerpo podrían variar secularmente, como por ejemplo una disminución en el tiempo del semieje, o un aumento de la excentricidad, fenómenos que podrían ser tan importantes que incluso el cuerpo pasaría de describir una órbita elíptica a una órbita parabólica, resultando expulsado del sistema. Por lo expuesto aquí podemos ver que estos comportamientos tendrán un efecto decisivo en la estructura y estabilidad del sistema (ver, por ejemplo, [10]). 
La variación de las acciones está fuertemente relacionada con el tamaño de la perturbación al sistema integrable ([12], [38], [46]). Para una perturbación suficientemente grande, las acciones presentarán una variación secular que se puede cuantificar fácilmente. De este resultado es posible determinar, por ejemplo, con qué velocidad están variando estas acciones. Por otro lado, para una perturbación más pequeña, la componente caótica del sistema disminuye, y la región sobre la cual las acciones pueden variar es más acotada. Esto produce como consecuencia que la difusión disminuya a medida que disminuye la perturbación. En el caso extremo, cuando la perturbación es nula, el sistema es integrable y, por consiguiente, las acciones son constantes. Luego, existirá un valor límite de la perturbación para el cual la variación secular de las acciones se manifiesta, pero su magnitud será lo suficientemente pequeña como para que dicha variación quede oculta a causa de las oscilaciones de dicha acción. La Fig. 4.I esquematiza un ejemplo de variación cuadrática de las acciones con respecto a su valor inicial $\left(\eta^{2}\right.$, la cual será definida más adelante en este capítulo) en función del número de iteraciones, que cuantifica el tamaño de la perturbación, para un mapa simpléctico 4D (que estudiaremos en el Capítulo 5).
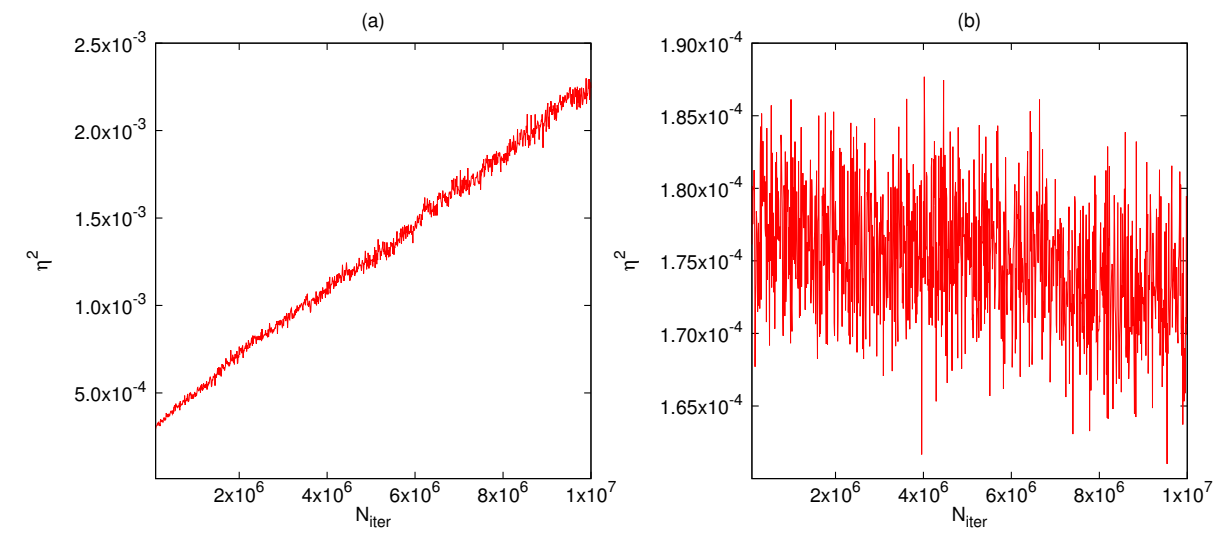

Figura 4.1: Gráficos de difusión para una dada órbita con dos valores distintos del parámetro perturbativo. En el panel (a) se utiliza un parámetro perturbativo cuyo tamaño es aproximadamente el doble que en el panel (b).

Como se verá más adelante, en el sistema que estudiaremos la frecuencia coincide con la acción $\left(\omega_{i} \equiv I_{i}\right)$. Luego, una resonancia, que es una relación entre frecuencias, la podemos expresar como esa misma relación entre acciones. Así lo haremos en este trabajo, en el que graficaremos las resonancias directamente en el espacio de acciones. 
En el panel (a) se muestra la variación de una acción a lo largo de una resonancia para un parámetro perturbativo relativamente alto, donde se puede observar que existe un crecimiento sistemático de la acción. En el panel (b), en cambio, se muestra la variación de la misma magnitud, para un parámetro más pequeño. En este gráfico, la difusión podría existir, pero sería tan pequeña que la variación secular queda oculta por las oscilaciones debidas a efectos de "deformación"del mapa (ver [41], pág. 63).

Esta diferencia en los comportamientos mostrados en la Fig. 4.1, producido por la variación del parámetro perturbativo, hace que resulte imprescindible la utilización de alguna herramienta que permita revelar el comportamiento difusivo en situaciones donde el parámetro perturbativo es lo suficientemente pequeño de modo que no permite observar la difusión en el conjunto de variables originales del sistema. La herramienta que utilizaremos son las formas normales, que se pueden definir como una o más transformaciones canónicas a un conjunto de variables ángulo-acción de manera que el mapa resultante tenga una forma que nos permita describir la dinámica del sistema de forma clara y sencilla. Esto haría que las oscilaciones se reduzcan considerablemente, y se pueda observar más claramente la difusión. Es una herramienta ampliamente utilizada cuando se quiere cuantificar la difusión para parámetros perturbativos muy pequeños ([18], [25], [26], [30], [29], [52], [68], entre otros), por lo cual dedicaremos este capítulo a su descripción.

En las secciones siguientes se explicará cómo calcular la transformación a la forma normal de un mapa simpléctico genérico 4D del tipo llamado cuasitorsional, pero para ello debemos introducir primero algunos conceptos básicos necesarios para el desarrollo de la transformación.

\subsection{DEFINICIONES Y CONCEPTOS BÁSICOS}

\subsubsection{Difusión}

Consideremos un sistema con $\mathrm{N}_{\mathrm{dim}}$ grados de libertad, ya sea un flujo hamiltoniano o un mapa simpléctico, definido por variables ángulo $x_{i} \mathrm{y}$ variables acción $J_{i}$, con $i=1, \ldots, N_{\text {dim }}$.

En el espacio de las acciones $J_{i}$, con $i=1, \ldots, N_{d i m}$, recordemos que la difusión se define como una variación secular de las acciones no perturbadas. Esta cantidad se cuantifica mediante el cálculo de un desplazamiento medio cuadrático de la acción con respecto a un valor representativo (el valor medio, el valor inicial, etc.). Para llevar a cabo este cálculo es necesario suponer que el tipo de difusión que presentan las acciones es del tipo llamado difusión normal ([3] ]). 
Bajo este régimen, para cuantificar la difusión, es estadísticamente equivalente considerar el promedio espacial y el promedio temporal. Esto es, podemos considerar un conjunto de condiciones iniciales y calcular su promedio espacial para cada instante de tiempo (como en [18], [42], [51]), o bien podemos considerar una única condición inicial con un tiempo de integración mayor que en el caso anterior, dividir este tiempo en intervalos de tamaño menor y calcular el promedio temporal sobre cada uno de esos intervalos ([10], [12]). En este trabajo vamos a emplear el primer método.

Una de las herramientas que podemos utilizar, basadas en el cálculo del promedio espacial, es la varianza (ver, por ejemplo, [18], [38], [51]).

Sea $J_{i}(t, j)$ la acción $J_{i}$ correspondiente al tiempo $t$ para la condición inicial $j$ de un conjunto de $\mathrm{N}_{c i}$ valores iniciales. La varianza se calcula como

$$
\sigma_{i}^{2}(t)=\left\langle\left(J_{i}(t)-\overline{J_{i}}(t)\right)^{2}\right\rangle=\frac{1}{N_{c i}} \sum_{j=1}^{N_{c i}}\left(J_{i}(t, j)-\overline{J_{i}}(t)\right)^{2},
$$

donde la sumatoria indica que se está promediando sobre el conjunto de condiciones iniciales, y $\overline{J_{i}}(t)$ es el valor medio de la acción $J_{i}$ en el conjunto para el tiempo $t$

$$
\overline{J_{i}}(t)=\frac{1}{N_{c i}} \sum_{j=1}^{N_{c i}} J_{i}(t, j) .
$$

La varianza (Ec. (4.I)) es un indicador de la difusión muy útil cuando se quiere estudiar un conjunto de condiciones iniciales que se dispersan en el espacio de las acciones, todas alrededor de un valor medio.

Otro indicador que puede utilizarse para cuantificar la difusión es la desviación cuadrática media (d.c.m. de aquí en más), que representamos como $\eta^{2}$, cuya expresión es

$$
\eta_{i}^{2}(t)=\frac{1}{N_{c i}}\left(\sum_{j=1}^{N_{c i}}\left(J_{i}(t, j)-J_{i}\left(t_{0}, j\right)\right)^{2}\right),
$$

donde $J_{i}\left(t_{0}, j\right)$ representa la acción $J_{i}$ de la j-ésima condición inicial del conjunto al tiempo inicial $t_{0}$.

Podemos notar que las definiciones de las Ecs. (4.1) y (4.3) son muy similares entre sí. La diferencia entre ellas es que, para la varianza estamos considerando el desplazamiento cuadrático con respecto al valor medio del conjunto $\overline{\mathrm{J}_{\mathfrak{i}}}(\mathrm{t})$, 
mientras que para la d.c.m. lo hacemos con respecto al valor inicial $J_{i}\left(t_{0}, j\right)$. En este trabajo, utilizaremos el cálculo de la d.c.m.

Si bien no se conoce a priori la forma que tiene la evolución de la difusión en el tiempo, comúnmente se supone (como en [18]) que ésta se comporta como una ley de potencias de la forma

$$
\eta^{2}(t) \approx \alpha t^{\beta}
$$

con $\alpha, \beta \in \mathbb{R}$ dos constantes a determinar.

De acuerdo al valor de $\beta$, se tienen distintos tipos de difusión ([18]). Si $\beta<1$ se obtiene la llamada subdifusión, si $\beta>1$ se llama superdifusión, y por último, si $\beta=1$ se obtiene lo que nos interesa en este trabajo, la difusión normal.

Un último concepto por ver es el de coeficiente de difusión. El coeficiente de difusión da una medida de la tasa a la cual se está produciendo la variación de las acciones. Como hemos mencionado anteriormente, para poder cuantificar la difusión, suponemos que ésta es normal. De esta manera, $\beta=1$ y la expresión de la Ec. (4.4) establece una dependencia lineal entre $\eta^{2}$ y el tiempo t, obteniendo una expresión de la forma

$$
\eta^{2}(t) \approx \mathcal{D} t
$$

donde la constante $\mathcal{D}$ (que se corresponde con $\alpha$ en la Ec. (4.4)) es el llamado coeficiente de difusión.

En las secciones siguientes de este capítulo estudiaremos la dependencia de la difusión con el tiempo en sistemas con perturbaciones de diferentes magnitudes, mediante el cálculo de $\eta^{2}(t)$. El objetivo de este estudio es estimar en qué intervalos de tiempo es posible obtener difusión normal, mediante el ajuste de una función lineal a la evolución de $\eta^{2}$, para luego estimar $\mathcal{D}$, a partir de la pendiente de dicho ajuste.

\subsubsection{El mapa de torsión}

Una vez introducido el concepto de mapa simpléctico (ver Sección 2.1.4) nos concentraremos en un tipo particular de éstos, los llamados twist maps, o "mapas de torsión". ${ }^{1}$

1 La definición que damos a continuación fue tomada de [59]. 
Consideremos primero mapas 2D, donde supondremos que el espacio de fases $(\theta, \mathrm{I})$ posee simetría cilíndrica, con $\theta$ siendo la coordenada angular e I una variable acción y que, por lo tanto, no está acotada. Sea

$$
\mathrm{T}:(\theta, \mathrm{I}) \rightarrow\left(\theta^{\prime}, \mathrm{I}^{\prime}\right)
$$

un mapa simpléctico de un espacio con simetría cilíndica a otro con la misma simetría, y supongamos que T es diferenciable. Luego T es un mapa de torsión (con una torsión a la derecha) si existe un $\mathrm{K}$ tal que

$$
\left.\frac{\mathrm{d} \theta^{\prime}}{\mathrm{dI}}\right|_{\theta} \geqslant K>0
$$

esto quiere decir que $\theta^{\prime}$ es una función monótonamente creciente de I. Consideremos, siguiendo a [59], la primera iteración de un conjunto de condiciones iniciales de la forma $\left(\theta_{0}, I\right)$, con $\theta_{0}$ constante (es decir, una línea vertical en el plano $(\theta, \mathrm{I}))$. Luego de la iteración, dicha línea se inclina hacia la derecha. Desde un punto de vista físico, la condición de torsión significa que la frecuencia angular depende de la acción. Por lo tanto, puntos con mayor acción (I) deberían moverse más lejos en ángulo $(\theta)$.

Suponiendo que el mapa es diferenciable, podemos considerar su acción sobre un vector tangente $(\delta \theta, \delta \mathrm{I})$ como

$$
\left[\begin{array}{l}
\delta \theta^{\prime} \\
\delta \mathrm{I}^{\prime}
\end{array}\right]=\left[\begin{array}{ll}
\frac{\partial \theta^{\prime}}{\partial \theta} & \frac{\partial \theta^{\prime}}{\partial \mathrm{I}} \\
\frac{\partial \mathrm{I}^{\prime}}{\partial \theta} & \frac{\partial \mathrm{I}^{\prime}}{\partial \mathrm{I}}
\end{array}\right]\left[\begin{array}{l}
\delta \theta \\
\delta \mathrm{I}
\end{array}\right]=M\left[\begin{array}{l}
\delta \theta \\
\delta \mathrm{I}
\end{array}\right] .
$$

Dado que el mapa preserva el volumen por ser simpléctico, el determinante de la matriz $M$ es $\operatorname{det}(M)=1$. La inversa linealizada del mapa (Ec. (4.6)) es representada por la derivada de $T^{-1}$ así como también por la inversa de $M$; luego

$$
M^{-1}=\left[\begin{array}{ll}
\frac{\partial \theta}{\partial \theta^{\prime}} & \frac{\partial \theta}{\partial \mathrm{I}^{\prime}} \\
\frac{\partial \mathrm{I}}{\partial \theta^{\prime}} & \frac{\partial \mathrm{I}}{\partial \mathrm{I}^{\prime}}
\end{array}\right]=\left[\begin{array}{cc}
\frac{\partial \mathrm{I}^{\prime}}{\partial \mathrm{I}} & -\frac{\partial \theta^{\prime}}{\partial \mathrm{I}} \\
-\frac{\partial \mathrm{I}^{\prime}}{\partial \theta} & \frac{\partial \theta^{\prime}}{\partial \theta}
\end{array}\right] .
$$

Comparando los elementos de ambas matrices, y recordando la Ec. (4.7) obtenemos que

$$
\left.\frac{\partial \theta}{\partial \mathrm{I}^{\prime}}\right|_{\theta^{\prime}}=-\left.\frac{\partial \theta^{\prime}}{\partial \mathrm{I}}\right|_{\theta} \leqslant-\mathrm{K}
$$


Esto nos dice que, si $\mathrm{T}$ es un mapa de torsión, entonces $\mathrm{T}^{-1}$ también lo es, pero uno con torsión a la izquierda. Notemos que $\mathrm{T}^{2}$ no tiene que ser necesariamente un mapa de torsión (donde $\mathrm{T}^{2}$ significa dos aplicaciones sucesivas del mapa $\mathrm{T}$ ), y de hecho no lo es por lo general, porque la línea inclinada puede torcerse lo suficiente en la segunda iteración, violando la condición de torsión ( $\mathrm{T}^{2}$ es un miembro de una clase más general de mapas, llamados mapas de inclinación (tilt maps), que no serán tratados en este trabajo).

\subsection{EL MAPA ESTÁNDAR RACIONAL ACOPLADO Y DESPLAZADO (CRSSM)}

El mapa que se estudiará en esta segunda parte del trabajo, construido a partir de dos mapas estándar ${ }^{2}$, los cuales están desplazados y luego acoplados, fue definido originalmente en [17]. Su nombre, CRSSM, es un acrónimo de su denominación en inglés (Coupled Rational Shifted Standard Map). Las ecuaciones que definen este mapa son

$$
\begin{aligned}
y_{1}^{\prime} & =y_{1}+\varepsilon_{y_{1}} f_{1}\left(x_{1}\right)+\gamma_{+} f_{3}\left(x_{1}+x_{2}\right)+\gamma_{-} f_{3}\left(x_{1}-x_{2}\right) \\
y_{2}^{\prime} & =y_{2}+\varepsilon_{y_{2}} f_{2}\left(x_{2}\right)+\gamma_{+} f_{3}\left(x_{1}+x_{2}\right)-\gamma_{-} f_{3}\left(x_{1}-x_{2}\right) \\
x_{1}^{\prime} & =x_{1}+\varepsilon_{y_{1}} y_{1}^{\prime} \\
x_{2}^{\prime} & =x_{2}+\varepsilon_{y_{2}} y_{2}^{\prime}
\end{aligned}
$$

con $x_{i} \in[0,2 \pi)$ variables ángulo e $y_{i} \in\left[0,2 \pi / \varepsilon_{y_{i}}\right)$ sus acciones conjugadas, con $i=1$, 2. Las funciones $f_{i}$ están definidas como

$$
f_{i}(x)=\frac{\operatorname{sen}\left(x+\varphi_{i}\right)}{1-\mu_{i} \cos (x)}-\Delta_{i}, \quad i=1,2,3,
$$

con

$$
\Delta_{i}=\frac{\mu_{i} \operatorname{sen}\left(\varphi_{i}\right)}{\sqrt{1-\mu_{i}{ }^{2}}+1-\mu_{i}{ }^{2}}, \quad i=1,2,3,
$$

donde $\Delta_{i}$ se fija de manera tal que las funciones $f_{i}$ tengan media cero. En la definición del mapa se ve que la denominación de acoplado se debe a los dos

2 Recordemos que el mapa estándar ([11]) es un mapa que preserva el área, cuya expresión viene dada por

$$
\begin{aligned}
& p^{\prime}=p+K \operatorname{sen}(x) \\
& x^{\prime}=x+p^{\prime},
\end{aligned}
$$

donde $(x, p)$ son variables canónicas conjugadas. 
términos $f_{3}\left(x_{1}+x_{2}\right)$ y $f_{3}\left(x_{1}-x_{2}\right)$, incorporados mediante los parámetros de acoplamiento $\gamma_{+}$y $\gamma_{-}$, respectivamente. Por otro lado, el carácter de racional resulta de la introducción del denominador $1-\mu_{i} \cos (x)$ donde, la utilización de parámetros $\mu_{i} \neq 0$, con $i=1,2,3$, hace que aparezcan todos los armónicos, y no solamente el sen $(x)$ como en el caso del mapa estándar. La introducción de los parámetros de fase $\varphi_{i}$, con $i=1,2,3$, como parte de los argumentos de las funciones trigonométricas modifican la simetría del mapa CRSSM en los ángulos. Por último, los parámetros $\varepsilon_{y_{i}}, i=1,2$, sencillamente representan los parámetros perturbativos del mapa estándar.

Para facilitar el análisis, se considerará una versión simplificada de este mapa, reduciendo la cantidad de parámetros libres. Por un lado se utilizarán fases nulas. Esto producirá que las funciones $f_{i}$ sean simétricas, por lo tanto tendrán media cero, y los valores de $\Delta_{i}$ serán todos nulos. También se considera la igualdad de los parámetros perturbativos $\left(\varepsilon_{y_{1}}, \varepsilon_{y_{2}}\right)$ y de los parámetros de acoplamiento $\left(\gamma_{+}, \gamma_{-}\right)$. Por último, en cuanto a los parámetros $\mu_{i}$ utilizaremos valores fijos para todos nuestros cálculos. En suma, el conjunto de parámetros utilizados es el siguiente

- $\varphi_{1}=\varphi_{2}=\varphi_{3}=0$

- $\varepsilon_{y_{1}}=\varepsilon_{y_{2}}=\varepsilon$

- $\gamma_{+}=\gamma_{-}=\gamma$

- $\mu_{1}=0,5, \mu_{2}=0,4, \mu_{3}=0,6$.

En la forma presentada en la Ec. (4.11) el dominio del mapa es la región $[0 ; 2 \pi) \times[0 ; 2 \pi)$ para los ángulos y $[0 ; 2 \pi / \varepsilon) \times[0 ; 2 \pi / \varepsilon)$ para las acciones. Renormalizaremos el mapa de manera que, en vez de utilizar un mapa cuyo dominio en las acciones tenga un tamaño que depende del parámetro $\varepsilon$, utilizaremos uno donde su dominio sea la región $[0 ; 2 \pi) \times[0 ; 2 \pi)$. Esta renormalización se logra mediante el cambio de variables $\tilde{y}_{i}=\varepsilon y_{i}$. Multiplicando por $\varepsilon$ las dos ecuaciones del mapa correspondientes a las acciones obtenemos

$$
\begin{aligned}
& \varepsilon y_{1}^{\prime}=\varepsilon y_{1}+\varepsilon\left(\varepsilon f_{1}\left(x_{1}\right)+\gamma\left[f_{3}\left(x_{1}+x_{2}\right)+f_{3}\left(x_{1}-x_{2}\right)\right]\right) \\
& \varepsilon y_{2}^{\prime}=\varepsilon y_{2}+\varepsilon\left(\varepsilon f_{2}\left(x_{2}\right)+\gamma\left[f_{3}\left(x_{1}+x_{2}\right)-f_{3}\left(x_{1}-x_{2}\right)\right]\right)
\end{aligned}
$$

$y$, reemplazando por la nueva variable $\tilde{y_{i}}$ en ambos miembros, se llega a

$$
\begin{aligned}
& \tilde{y}_{1}^{\prime}=\tilde{y}_{1}+\varepsilon^{2} f_{1}\left(x_{1}\right)+\varepsilon \gamma\left[f_{3}\left(x_{1}+x_{2}\right)+f_{3}\left(x_{1}-x_{2}\right)\right] \\
& \tilde{y}_{2}^{\prime}=\tilde{y}_{2}+\varepsilon^{2} f_{2}\left(x_{2}\right)+\varepsilon \gamma\left[f_{3}\left(x_{1}+x_{2}\right)-f_{3}\left(x_{1}-x_{2}\right)\right]
\end{aligned}
$$


Para las ecuaciones de los ángulos de la Ec. (4.II), la renormalización es inmediata. Simplemente reemplazamos el término perturbativo y obtenemos

$$
\begin{aligned}
& x_{1}^{\prime}=x_{1}+\tilde{y}_{1}^{\prime} \\
& x_{2}^{\prime}=x_{2}+\tilde{y}_{2}^{\prime} .
\end{aligned}
$$

Por motivos de simplicidad en la escritura de las ecuaciones, haremos abuso de la notación y escribiremos a $\tilde{y}_{i}$ simplemente como $y_{i}$, pero recordemos que ésta es la variable normalizada. Aplicando esta notación a las Ecs. (4.15) y (4.16), podemos reescribir el mapa normalizado como

$$
\begin{aligned}
& \left.y_{1}^{\prime}=y_{1}+\varepsilon^{2} f_{1}\left(x_{1}\right)+\varepsilon \gamma\left[f_{3}\left(x_{1}+x_{2}\right)+f_{3}\left(x_{1}-x_{2}\right)\right]\right) \\
& \left.y_{2}^{\prime}=y_{2}+\varepsilon^{2} f_{2}\left(x_{2}\right)+\varepsilon \gamma\left[f_{3}\left(x_{1}+x_{2}\right)-f_{3}\left(x_{1}-x_{2}\right)\right]\right) \\
& x_{1}^{\prime}=x_{1}+y_{1}^{\prime} \\
& x_{2}^{\prime}=x_{2}+y_{2}^{\prime} .
\end{aligned}
$$

Es fácil comprobar que sobre la forma de la Ec. (4.17), el mapa después de la nueva definición de variables, mantiene su propiedad de ser simpléctico. Además hemos reducido el número de parámetros libres de 10 a 2, quedando solamente $\varepsilon$ y $\gamma$.

\subsubsection{Selección de las cantidades a calcular}

Para estudiar la difusión es preciso calcular la variación de las acciones en el tiempo. Como hemos visto en la Sección 4.1.I, dos de las herramientas que suelen utilizarse para cuantificar la difusión son la varianza $\sigma^{2}$ (como en [18]) y la d.c.m. $\eta^{2}$ ([38], [51]).

Para cuantificar la difusión calcularemos la variación de la llamada "acción rápida", $I_{f}$, que representa la acción en la dirección de la resonancia. Para ello hacemos un cambio de variables $\left(y_{1}, y_{2}\right) \rightarrow\left(I_{R}, I_{f}\right)$, donde $I_{f}$ es la mencionada acción rápida, e $\mathrm{I}_{\mathrm{R}}$ es la acción resonante, orientada en la dirección perpendicular a la resonancia. Esta transformación se realiza reescribiendo el conjunto de acciones $\left(y_{1}, y_{2}\right)$ como una combinación lineal de las dos nuevas

$$
\begin{aligned}
& y_{1}=k_{1} I_{R}+m_{1} I_{f} \\
& y_{2}=k_{2} I_{R}+m_{2} I_{f}
\end{aligned}
$$


donde $k_{i}, m_{i} \in \mathbb{Z}, i=1,2$. Aquí $k_{r}=\left(k_{1}, k_{2}\right)$ son valores enteros que, junto con el valor entero $k_{3}$, satisfacen la condición de resonancia

$$
k_{1} \omega_{1}+k_{2} \omega_{2}+k_{3} 2 \pi=0,
$$

con la componente $k_{3}$ asociada a un incremento en el tiempo. Dado que en este mapa la frecuencia no perturbada coincide con la acción $\left(\omega_{i} \equiv y_{i}\right)^{3}$, en adelante utilizaremos ambas variables de forma equivalente. Si reemplazamos las frecuencias en la Ec. (4.19) y resolvemos para y2 la condición de resonancia adquiere la forma de una recta

$$
y_{2}=-\frac{k_{1}}{k_{2}} y_{1}-\frac{k_{3}}{k_{2}}
$$

Para este trabajo consideraremos nuestros estudios sobre la resonancia $1: 1$, que pasa por el origen del plano $\left(y_{1}, y_{2}\right)$. Esto hace que $k_{3}=0$ y las frecuencias cumplen la relación $\omega_{1}=\omega_{2}=\omega$. Luego, de la condición

$$
\mathbf{k}_{\mathrm{r}} \cdot(\omega, \omega)=0,
$$

obtenemos que $\mathbf{k}_{\mathrm{r}}=(1,-1)$. Dado que $\mathrm{I}_{\mathrm{f}}$ representa la acción en la dirección de la resonancia, perpendiculares entre sí (Ec. (4.18)), luego $\mathbf{k}_{\mathrm{r}} \cdot \mathbf{m}=\mathrm{k}_{1} \mathrm{~m}_{1}+$ $k_{2} m_{2}=0$, con $\mathbf{m} \neq \mathbf{o}$. Para la resonancia que hemos considerado, tenemos que $\mathrm{m}_{1}=\mathrm{m}_{2}$.

Estos valores de $m_{i}$, con $i=1,2$ son arbitrarios. Por simplicidad, tomaremos $\mathrm{m}_{1}=\mathrm{m}_{2}=1$. Si reemplazamos los valores de $\mathbf{k}_{\mathbf{r}} \mathbf{y} \mathbf{m}$ en (4.18) obtenemos

$$
\begin{aligned}
& y_{1}=I_{R}+I_{f} \\
& y_{2}=-I_{R}+I_{f}
\end{aligned}
$$

$y$, resolviendo para $I_{f}$, resulta $I_{f}=\frac{y_{1}+y_{2}}{2}$. Como el valor en el denominador es una constante, podemos redefinir a $I_{f}$ como $I_{f}=y_{1}+y_{2}$ sin producir una alteración en su dinámica.

Una vez definida la variable $I_{f}$, lo que queremos calcular exactamente es cuánto se desvía ésta de su valor inicial $\mathrm{I}_{\mathrm{fo}}=\mathrm{y}_{10}+\mathrm{y}_{20}$ por medio de la d.c.m. De aquí en adelante, cuando se mencione la cantidad $\eta^{2}$, hará referencia a la d.c.m. en esta dirección.

3 La frecuencia $\omega_{i}$ se define como $\omega_{i}^{\prime}=\frac{d x_{i}^{\prime}}{d t}$. Dado que para este mapa $d t=1 \mathrm{y}$ es posible escribir $\mathrm{d} x_{i}^{\prime}=x_{i}^{\prime}-x_{i}$, a partir de las expresiones para los ángulos en la Ec. (4.17) obtenemos que $\omega_{i}^{\prime}=y_{i}^{\prime}$ $y$, de manera análoga, $\omega_{i}=y_{i}$. 
Para escoger el número de iteraciones con el cual se va a realizar el cálculo, consideremos el siguiente escenario. En un sistema galáctico, podría considerarse como un período un tiempo de cruce, el cual tiene una duración de $10^{7}-10^{8}$ años. Si su estudio se realiza mediante la implementación de un mapa, una iteración de éste equivale a uno de estos períodos característicos por lo que, en $10^{3}-10^{4}$ iteraciones, el tiempo transcurrido sería equivalente a un tiempo de Hubble. Por lo tanto, considerar un número de iteraciones mayor que éste carece de sentido para estudios difusivos. En cambio, si consideramos un sistema planetario (por ej. el Sistema Solar), su período característico podría ser un período de Júpiter, que se puede aproximar como 10 años. Luego, al considerar $10^{7}-10^{8}$ iteraciones, el tiempo transcurrido es, aproximadamente, la edad del Sistema Solar, por lo que carece de sentido considerar un número de iteraciones mayor. Por consiguiente, para que nuestros cálculos tengan significancia en un amplio rango de aplicaciones, utilizaremos un total de $\mathrm{N}_{\text {iter }}=10^{7}$ iteraciones, lo cual es suficiente para el estudio de la variación secular de las acciones.

\subsection{ESTIMACIÓN DE LA DIFUSIÓN PARA DISTINTAS MAGNitUdes DE LA PERTURBACIÓN}

En la Fig. 4.I podemos ver cómo es el comportamiento de la difusión para un conjunto de condiciones iniciales, ubicados en la capa estocástica de una resonancia, considerando un sistema con perturbaciones de tamaños diferentes. Este conjunto, compuesto por $10^{3}$ condiciones iniciales, fueron tomadas para el CRSSM sobre la capa estocástica de la resonancia $1: 1$, en un segmento de recta de longitud $10^{-9}$ orientada de forma perpendicular a ésta. Se evaluaron durante $\mathrm{N}_{\text {iter }}=10^{7}$ iteraciones, fijando el valor de $\varepsilon=0,1$ y para dos valores distintos del parámetro $\gamma: 0,1$ en el panel (a) y 0,0545 en el panel (b).

De la comparación entre estas gráficas podemos inferir que, cuando la perturbación es suficientemente pequeña, si existe un crecimiento de la d.c.m. éste es más pequeño que las amplitudes de las oscilaciones, dificultando la detección de la difusión. Una solución a este problema podría obtenerse de iterar las ecuaciones del mapa a tiempos mayores, esto es $10^{8}$ ó $10^{9}$. El aspecto negativo de esta técnica es que el tiempo de cómputo insumido se incrementa considerablemente.

Existen técnicas numéricas que reducen estas oscilaciones, requiriendo poco esfuerzo computacional extra, sin necesidad de aumentar el número de iteraciones. Una de ellas consiste en considerar el cálculo de la d.c.m. pero no sobre todo el espacio de fases, sino tomando solamente aquellos puntos que se encuentran en una región $V$ cercana a la superficie de sección del mapa, es decir $\left|x_{1}\right|+\left|x_{2}\right| \leqslant \delta$ donde $\delta$ es un parámetro libre que define el espesor de esa región (ver [38], [42], [44], [51]). Como no todas las condiciones iniciales se encontrarán 
al mismo tiempo en esta región, para un dado tiempo $t$, podemos dividir nuestro intervalo de iteración en subintervalos de tamaño $\Delta t, y$ calcular la cantidad

$$
\hat{\eta}_{i}^{2}(k \Delta t)=\frac{1}{M_{k}}\left(\sum_{j:\left|x_{1}(t, j)\right|+\left|x_{2}(t, j)\right| \in V}\left(J_{i}(t, j)-J_{i}\left(t_{0}, j\right)\right)^{2}\right),
$$

donde $M_{k}$ es el número de puntos que se encuentran en la región $V$ en el intervalo de $[(k-1) \Delta t: k \Delta t]$ iteraciones. La sumatoria a tiempo $t$ se debe realizar sobre todos los valores $j$ tal que la órbita $j$-ésima del conjunto considerado se encuentre en la región $V$, es decir

En la Ec. (4.23) ver que

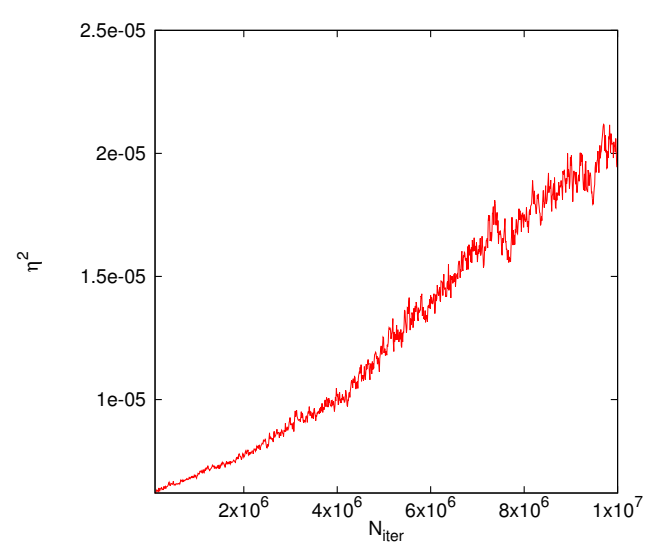

Figura 4.2: Evolución de $\hat{\eta}^{2}$ con respecto al número de iteraciones para $\gamma=0,0545$ considerando solamente los puntos que cumplen $\left|x_{1}\right|+\left|x_{2}\right| \leqslant \delta$, con $\delta=0,1$.

La Fig. 4.2 muestra la evolución temporal de la cantidad $\hat{\eta}^{2}(t)$ para $\gamma=$ 0,0545 , tomando como espesor de la región $V$ un valor relativamente grande, $\delta=0,1$, y fijando el tamaño del intervalo $\Delta t=10^{4}$. Comparando con el panel (b) de la Fig 4.I vemos que, luego de aplicar la técnica descripta, es posible advertir un comportamiento secular definido, similar al mostrado en el panel (a) de la Fig. 4.I. Efectivamente, al descartar los puntos que se encuentran fuera de la región $\mathrm{V}$, eliminamos los puntos que introducían las oscilaciones de mayor amplitud, permitiendo ahora la estimación de $\mathcal{D}$.

Al considerar un valor de $\gamma$ más pequeño, por ejemplo $\gamma=0,0264$ (Fig. 4.3) vemos que, como era de esperar, al considerar todos los puntos del espacio de fases (panel (a)), no es posible detectar una variación secular. En cuanto a la variación estimada con los puntos pertenecientes a $V($ panel (b)), si bien es posible observar un comportamiento secular definido, tamaño de las oscilaciones en esta figura es mayor con respecto a la aplicación de esta técnica para 
el caso de $\gamma=0,0545$ (Fig. 4.2). Este aumento es producido por el hecho que, al considerar un parámetro perturbativo más pequeño, la tasa de crecimiento de la d.c.m. ha disminuido con respecto a la observada para $\gamma=0,0545$, y las oscilaciones producidas por considerar puntos pertenecientes a una región $\mathrm{V}$ con $\delta=0,1$, comienzan a cobrar mayor importancia. Luego, para sortear este inconveniente, será necesario reducir el espesor de la región $V$ para considerar puntos más cercanos a la superficie de sección, y así mejorar la estimación de la d.c.m. En los paneles restantes de la Fig. 4.3, podemos ver el resultado del cálculo de la d.c.m. considerando diferentes valores de $\delta: 5 \times 10^{-2}$ (panel (c)), $10^{-2}$ (panel (d)) y $10^{-3}(\operatorname{panel}(\mathrm{e}))$.

Al efectuar la primera reducción del valor de $\delta$ a 0,05 vemos que las oscilaciones en la curva disminuyen. Lo mismo ocurre cuando pasamos a $\delta=0,01$. Sin embargo, al considerar un valor de $\delta$ muy pequeño, las oscilaciones crecen. Este efecto es debido a que, al disminuir el valor de $\delta$, se reduce la cantidad de puntos que permanecen en $V$, dando como resultado una estimación de la d.c.m. con mayor error que para un espesor un orden de magnitud mayor.

Este es otro inconveniente que presenta esta técnica. Al disminuir el valor del parámetro perturbativo $\gamma$, para poder detectar la variación secular de las acciones es necesario, o bien aumentar el tiempo de iteración (con un consiguiente aumento en los tiempos de cómputo) o bien reducir el tamaño de la región cercana a la superficie de sección, teniendo como consecuencia la reducción de puntos y el consiguiente aumento de los errores.

Por lo tanto, vemos conveniente contar con otra herramienta que nos permita estimar la difusión para valores perturbativos considerablemente pequeños. Esta herramienta, que veremos a continuación, son las formas normales. A diferencia de técnicas como la vista aquí que se basan en la selección de determinados puntos de la serie temporal, las formas normales consisten en una sucesión de transformaciones canónicas de las variables originales que definen el mapa en un nuevo conjunto de variables capaces de representar la dinámica del sistema de una manera más clara y sencilla.

La utilidad de la implementación de las formas normales no se restringe únicamente a su utilización como una herramienta para conseguir la reducción de las oscilaciones, en la estimación de la d.c.m. en escenarios con valores perturbativos pequeños, sino que tienen muchas otras aplicaciones. Por ejemplo, para poder estimar el valor del coeficiente de difusión de manera teórica, utilizando la fórmula de Chirikov ([12], [18]), es necesario contar con los coeficientes de los términos del desarrollo de Fourier de las ecuaciones del sistema. Las formas normales, logran representar las ecuaciones diferenciales de un flujo hamiltoniano (o las ecuaciones de diferencias en un mapa) como un desarrollo de Fourier, pudiendo utilizarse sus coeficientes para el cálculo teórico de $\mathcal{D}$. 

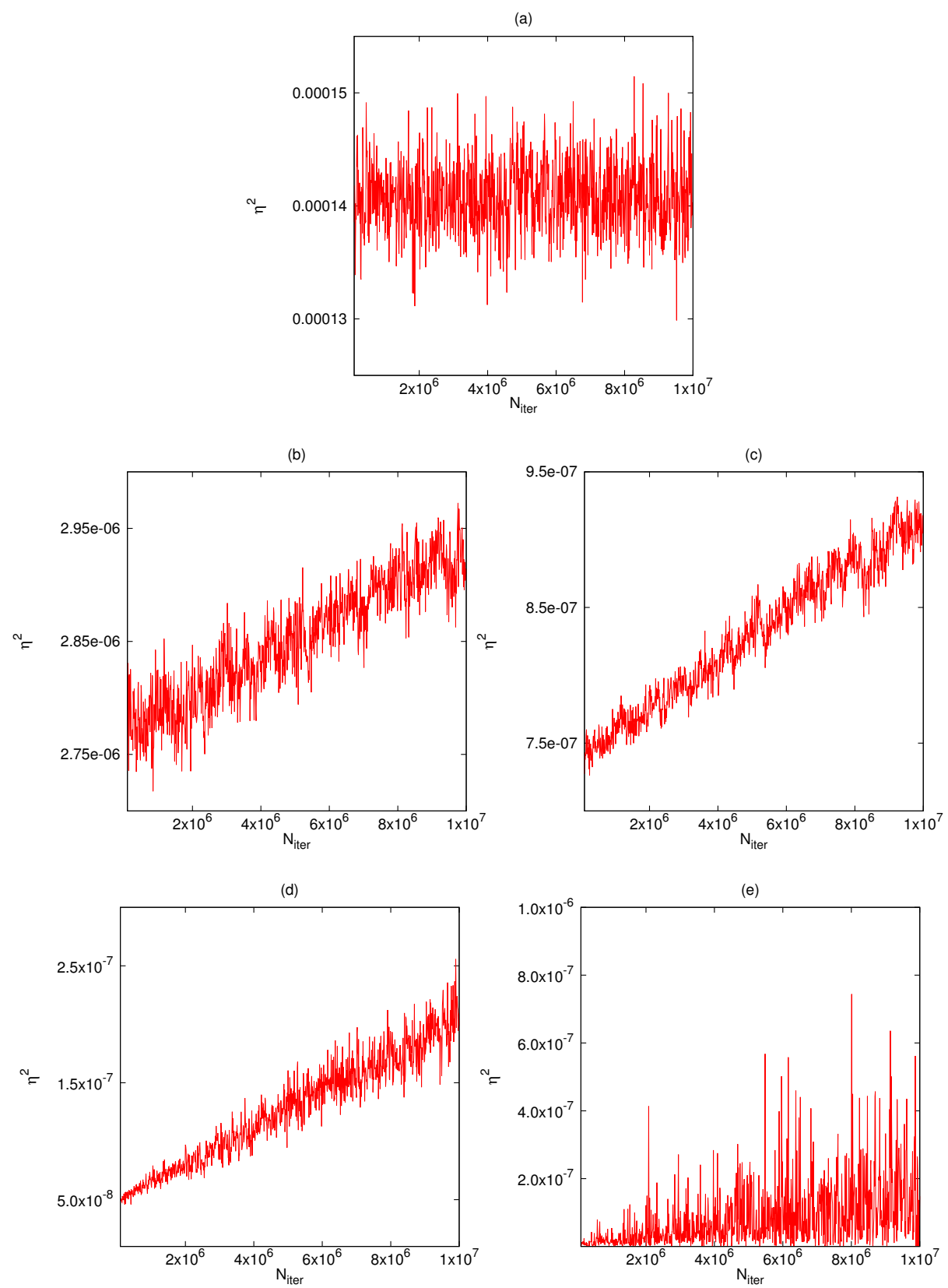

Figura 4.3: Evolución de $\eta^{2}$ con respecto al número de iteraciones para $\gamma=0,0264$ (a). Luego, considerando solamente los puntos que cumplen $\left|x_{1}\right|+\left|x_{2}\right| \leqslant \delta$, con $\delta=0,1(b), \delta=0,05$ (c), $\delta=0,01$ (d) y $\delta=0,001$ (e). 


\subsection{MÉTOdo PARA EL CÁlCULO DE LA FORMA NORMAL EN MAPAS}

En esta sección explicaremos el método para el cálculo de la forma normal de un mapa 4D simpléctico cuasi torsional genérico. El método desarollado en este capítulo solamente es válido si el punto central alrededor del cual se construye la transformación se encuentra en una resonancia simple 4 Para el desarrollo del algoritmo de la transformación a la forma normal, nos hemos basado en [30].

\subsubsection{Selección y preparación del mapa}

Por definición, un mapa simpléctico 4D cuasi-torsional es un mapa de la forma

$$
\left\{\begin{array}{l}
y_{1}^{\prime}=F_{1}\left(y_{1}, y_{2}, x_{1}, x_{2}\right)=y_{1}+\varepsilon_{1} \tilde{F}_{1}\left(y_{1}, y_{2}, x_{1}, x_{2}\right) \\
y_{2}^{\prime}=F_{2}\left(y_{1}, y_{2}, x_{1}, x_{2}\right)=y_{2}+\varepsilon_{2} \tilde{F}_{2}\left(y_{1}, y_{2}, x_{1}, x_{2}\right) \\
x_{1}^{\prime}=F_{3}\left(y_{1}, y_{2}, x_{1}, x_{2}\right)=x_{1}+\Omega_{1}\left(y_{1}, y_{2}\right)+\varepsilon_{3} \tilde{F}_{3}\left(y_{1}, y_{2}, x_{1}, x_{2}\right) \\
x_{2}^{\prime}=F_{4}\left(y_{1}, y_{2}, x_{1}, x_{2}\right)=x_{2}+\Omega_{2}\left(y_{1}, y_{2}\right)+\varepsilon_{4} \tilde{F}_{4}\left(y_{1}, y_{2}, x_{1}, x_{2}\right)
\end{array}\right.
$$

donde $y_{1}, y_{2}$ son las acciones, $x_{1}, x_{2}$ son sus ángulos conjugados, $\Omega_{\mathfrak{i}}\left(y_{1}, y_{2}\right)$ son funciones únicamente de los ángulos, y $\varepsilon_{i} \tilde{F}_{i}(i=1, \ldots, 4)$ son funciones pequeñas que pueden ser expresadas como series de Fourier, con términos de la forma

$$
C_{k_{1}, k_{2}}\left(y_{1}, y_{2}\right) e^{i\left(k_{1} x_{1}+k_{2} x_{2}\right)},
$$

con $k_{1}, k_{2} \in \mathbb{Z}$. El tamaño de $\varepsilon_{i} \tilde{F}_{i}$ permite considerar el mapa de la ec. (4.24) como un mapa de torsión, donde $\varepsilon_{i} \tilde{F}_{i}$ son considerados términos perturbativos del mapa. Todas las funciones $\tilde{F}_{i}$ y $\Omega_{j}$ deben cumplir ciertas condiciones de manera que el mapa resulte simpléctico. Dichas condiciones son: $\left\{x_{i}{ }^{\prime}, x_{j}{ }^{\prime}\right\}=0$, $\left\{y_{i}^{\prime}, y_{j}{ }^{\prime}\right\}=0,\left\{x_{i}{ }^{\prime}, y_{j}{ }^{\prime}\right\}=\delta_{i j}$, donde $\{f, g\}$ es el corchete de Poisson ${ }^{5}$ entre las funciones $\mathrm{f} \mathrm{y} g$.

Dado que la difusión que se quiere cuantificar es un fenómeno local (es decir, que la acción no produzca una variación lo suficientemente grande como para

4 Denominamos a una resonancia como resonancia simple cuando estamos ubicados en un punto de la resonancia, en el espacio de las acciones, tal que un pequeño entorno de éste se encuentra lejos de un cruce con otras resonancias de órdenes bajos.

5 Recordemos que el corchete de Poisson entre dos funciones $f$ y $g$, ambas dependientes de las variables $\left(y_{1}, y_{2}, x_{1}, x_{2}\right)$ tiene la forma

$$
\{f, g\}=\frac{\partial f}{\partial x_{1}} \frac{\partial g}{\partial y_{1}}+\frac{\partial f}{\partial x_{2}} \frac{\partial g}{\partial y_{2}}-\frac{\partial f}{\partial y_{1}} \frac{\partial g}{\partial x_{1}}-\frac{\partial f}{\partial y_{2}} \frac{\partial g}{\partial x_{2}}
$$


transitar regiones donde su dinámica varíe mucho, y sea necesario implementar otra herramienta), su estudio se va a realizar en las vecindades de un punto del espacio de las acciones, que llamaremos $\left(y_{1}^{*}, y_{2}^{*}\right)$. Luego de seleccionar el punto $\left(y_{1}^{*}, y_{2}^{*}\right)$, reescribimos las funciones $\tilde{F}_{i}$ como un desarrollo de Taylor de las variables acción alrededor de este punto,

$$
\begin{aligned}
\tilde{F}_{1}\left(\mathbf{v}_{\mathcal{F}}\right)= & \tilde{F}_{1}\left(\mathbf{v}_{\mathcal{F}}^{*}\right)+ \\
& \left.\sum_{n=1}^{\infty} \sum_{\substack{s_{1}, s_{2}=0 \\
s_{1}+s_{2}=n}}^{\infty} \frac{1}{s_{1} ! s_{2} !} \frac{\partial^{n} \tilde{F}_{1}\left(\mathbf{v}_{\mathcal{F}}\right)}{\partial y_{1}^{s_{1}} \partial y_{2}^{s_{2}}}\right|_{\mathbf{v}_{\mathcal{F}}^{*}}\left(y_{1}-y_{1}^{*}\right)^{s_{1}}\left(y_{2}-y_{2}^{*}\right)^{s_{2}} \\
\tilde{\mathrm{F}}_{2}\left(\mathbf{v}_{\mathcal{F}}\right)= & \tilde{\mathrm{F}}_{2}\left(\mathbf{v}_{\mathcal{F}}^{*}\right)+ \\
& \left.\sum_{n=1}^{\infty} \sum_{\substack{s_{1}, s_{2}=0 \\
s_{1}+s_{2}=n}}^{\infty} \frac{1}{s_{1} ! s_{2} !} \frac{\partial^{n} \tilde{F}_{2}\left(\mathbf{v}_{\mathcal{F}}\right)}{\partial y_{1}^{s_{1}} \partial y_{2}^{s_{2}}}\right|_{\mathbf{v}_{\mathcal{F}}^{*}}\left(y_{1}-y_{1}^{*}\right)^{s_{1}}\left(y_{2}-y_{2}^{*}\right)^{s_{2}} \\
\tilde{F}_{3}\left(\mathbf{v}_{\mathcal{F}}\right)= & \tilde{F}_{3}\left(\mathbf{v}_{\mathcal{F}}^{*}\right)+ \\
& \left.\sum_{n=1}^{\infty} \sum_{\substack{s_{1}, s_{2}=0 \\
s_{1}+s_{2}=n}}^{\infty} \frac{1}{s_{1} ! s_{2} !} \frac{\partial^{n} \tilde{F}_{3}\left(\mathbf{v}_{\mathcal{F}}\right)}{\partial y_{1}^{s_{1}} \partial y_{2}^{s_{2}}}\right|_{\mathbf{v}_{\mathcal{F}}^{*}}\left(y_{1}-y_{1}^{*}\right)^{s_{1}}\left(y_{2}-y_{2}^{*}\right)^{s_{2}} \\
\tilde{F}_{4}\left(\mathbf{v}_{\mathcal{F}}\right)= & \tilde{F}_{4}\left(\mathbf{v}_{\mathcal{F}}^{*}\right)+ \\
& \left.\sum_{n=1}^{\infty} \sum_{\substack{s_{1}, s_{2}=0 \\
s_{1}+s_{2}=n}}^{\infty} \frac{1}{s_{1} ! s_{2} !} \frac{\partial^{n} \tilde{F}_{4}\left(\mathbf{v}_{\mathcal{F}}\right)}{\partial y_{1}^{s_{1}} \partial y_{2}^{s_{2}}}\right|_{\mathbf{v}_{\mathcal{F}}^{*}}\left(y_{1}-y_{1}^{*}\right)^{s_{1}}\left(y_{2}-y_{2}^{*}\right)^{s_{2}},(4.26)
\end{aligned}
$$

con $\mathbf{v}_{\mathcal{F}}=\left(y_{1}, y_{2}, x_{1}, x_{2}\right)$ y $\mathbf{v}_{\mathcal{F}}^{*}=\left(y_{1}^{*}, y_{2}^{*}, x_{1}, x_{2}\right)$. Dado que las funciones $\tilde{\mathrm{F}}_{i}$ se pueden expresar como series de Fourier, sus derivadas parciales también podrán ser reescritas como series de Fourier

$$
\begin{aligned}
& \left.\frac{1}{s_{1} ! s_{2} !} \frac{\partial^{n} \tilde{F}_{j}\left(\mathbf{v}_{\mathcal{F}}\right)}{\partial y_{1}^{s_{1}} \partial y_{2}^{s_{2}}}\right|_{\mathbf{v}_{\mathcal{F}}^{*}}= \\
& \sum_{k_{1}, k_{2}=-\infty}^{\infty} \mathcal{C}_{j, k_{1}, k_{2}}\left(y_{1}^{*}, y_{2}^{*}\right) e^{i\left(k_{1} x_{1}+k_{2} x_{2}\right)}
\end{aligned}
$$

donde $j=1, \ldots, 4, \mathfrak{e}_{j, k_{1}, k_{2}} \in \mathbb{C}$ son constantes, $y k_{1}, k_{2} \in \mathbb{Z}$. Para simplificar la notación utilizada, se introduce un nuevo par de variables que, por definición, son cantidades pequeñas:

$$
\begin{aligned}
& J_{1}=y_{1}-y_{1}^{*} \\
& J_{2}=y_{2}-y_{2}^{*} .
\end{aligned}
$$


Dado que existe una gran similitud entre las variables $y_{i}$ y $J_{i}$, si bien estas últimas no son acciones, por simplicidad también las llamaremos acciones. Si se reemplazan en las Ecs. (4.26)

$$
\begin{aligned}
\tilde{F}_{1}\left(J_{1}, J_{2}, x_{1}, x_{2}\right)= & \tilde{F}_{1}\left(y_{1}^{*}, y_{2}^{*}, x_{1}, x_{2}\right)+ \\
& \left.\sum_{n=1}^{\infty} \sum_{s_{1}+s_{2}=n} \frac{1}{s_{1} ! s_{2} !} \frac{\partial^{n} \tilde{F}_{j}\left(\mathbf{v}_{\mathcal{F}}\right)}{\partial y_{1}^{s_{1}} \partial y_{2}^{s_{2}}}\right|_{\mathbf{v}_{\mathcal{F}}^{*}} \\
\tilde{F}_{2}\left(J_{1}, J_{2}, x_{1}, x_{2}\right)= & \tilde{F}_{2}\left(y_{1}^{*}, y_{2}^{*}, x_{1}, x_{2}\right)+ \\
& \left.\sum_{n=1}^{\infty} \sum_{s_{1}+s_{2}=n} \frac{1}{s_{1} ! s_{2} !} \frac{\partial^{n} \tilde{F}_{j}\left(\mathbf{v}_{\mathcal{F}}\right)}{\partial y_{1}^{s_{1}} \partial y_{2}^{s_{2}}}\right|_{\mathbf{v}_{\mathcal{F}}^{*}} \\
\tilde{F}_{3}\left(J_{1}, J_{2}, x_{1}, x_{2}\right)= & \tilde{F}_{3}\left(y_{1}^{*}, y_{2}^{*}, x_{1}, x_{2}\right)+ \\
& \left.\sum_{n=1}^{\infty} \sum_{s_{1}+s_{2}=n} \frac{1}{s_{1} ! s_{2} !} \frac{\partial^{n} \tilde{F}_{j}\left(\mathbf{v}_{\mathcal{F}}\right)}{\partial y_{1}^{s_{1}} \partial y_{2}^{s_{2}}}\right|_{\mathbf{v}_{\mathcal{F}}^{*}} \\
\tilde{F}_{4}\left(J_{1}, J_{2}, x_{1}, x_{2}\right)= & \tilde{F}_{4}\left(y_{1}^{*}, y_{2}^{*}, x_{1}, x_{2}\right)+ \\
& \left.\sum_{n=1}^{\infty} \sum_{s_{1}+s_{2}=n} \frac{1}{s_{1} ! s_{2} !} \frac{\partial^{n} \tilde{F}_{j}\left(\mathbf{v}_{\mathcal{F}}\right)}{\partial y_{1}^{s_{1}} \partial y_{2}^{s_{2}}}\right|_{\mathbf{v}_{\mathcal{F}}^{*}} .
\end{aligned}
$$

Para las ecuaciones de los ángulos, como $\Omega_{1}$ y $\Omega_{2}$ dependen de $\left(y_{1}, y_{2}\right)$ también deben desarrollarse en series de potencias en torno al punto $\left(y_{1}^{*}, y_{2}^{*}\right)$, obteniendo, luego de reemplazar la Ec. (4.28)

$$
\begin{aligned}
\Omega_{1}\left(J_{1}, J_{2}\right)= & \Omega_{1}\left(y_{1}^{*}, y_{2}^{*}\right)+ \\
& \left.\sum_{n=1}^{\infty} \sum_{s_{1}+s_{2}=n} \frac{1}{s_{1} ! s_{2} !} \frac{\partial^{n} \Omega_{1}}{\partial y_{1}^{s_{1}} \partial y_{2}^{s_{2}}}\right|_{\left(y_{1}^{*}, y_{2}^{*}\right)} J_{1}^{s_{1}} J_{2}^{s_{2}}, \\
\Omega_{2}\left(J_{1}, J_{2}\right)= & \Omega_{2}\left(y_{1}^{*}, y_{2}^{*}\right)+ \\
& \left.\sum_{n=1}^{\infty} \sum_{s_{1}+s_{2}=n} \frac{1}{s_{1} ! s_{2} !} \frac{\partial^{n} \Omega_{2}}{\partial y_{1}^{s_{1}} \partial y_{2}^{s_{2}}}\right|_{\left(y_{1}^{*}, y_{2}^{*}\right)} J_{1}^{s_{1}} J_{2}^{s_{2}} .
\end{aligned}
$$

Llamemos $\omega_{i}^{*}=\Omega_{i}\left(y_{1}^{*}, y_{2}^{*}\right)$. El resto de los términos contienen solamente potencias de $\mathrm{J}_{1} \mathrm{y} \mathrm{J}_{2}$, por lo tanto éstos pueden ser reescritos como términos de un desarrollo de Fourier sin dependencia en los ángulos, es decir, $k_{1}=k_{2}=0$.

Definiendo $J_{i}^{\prime}=y_{i}^{\prime}-y_{i}^{*}$, reescribimos las ecuaciones del mapa (Ec. (4.24)) aplicando el cambio de variables de la Ec. (4.28). Debido a este reemplazo, aparecerá a cada lado de la igualdad el término $y_{i}^{*}$, cancelándose entre sí.

Finalmente, el mapa que se obtiene es 


$$
\begin{aligned}
& \mathrm{J}_{1}^{\prime}=\mathrm{J}_{1}+\sum_{n=0}^{\infty} \sum_{s_{1}+s_{2}=n} \sum_{k_{1}, k_{2}=-\infty}^{\infty} e_{1, s_{1}, s_{2}, k_{1}, k_{2}} J_{1}^{s_{1}} J_{2}^{s_{2}} e^{i\left(k_{1} x_{1}+k_{2} x_{2}\right)} \\
& J_{2}^{\prime}=J_{2}+\sum_{n=0}^{\infty} \sum_{s_{1}+s_{2}=n} \sum_{k_{1}, k_{2}=-\infty}^{\infty} e_{2, s_{1}, s_{2}, k_{1}, k_{2}} J_{1}^{s_{1}} J_{2}^{s_{2}} e^{i\left(k_{1} x_{1}+k_{2} x_{2}\right)} \\
& x_{1}^{\prime}=x_{1}+\omega_{1}^{*}+\sum_{n=0}^{\infty} \sum_{s_{1}+s_{2}=n} \sum_{k_{1}, k_{2}=-\infty}^{\infty} e_{3, s_{1}, s_{2}, k_{1}, k_{2}} J_{1}^{s_{1}} J_{2}^{s_{2}} e^{i\left(k_{1} x_{1}+k_{2} x_{2}\right)} \\
& x_{2}^{\prime}=x_{2}+\omega_{2}^{*}+\sum_{n=0}^{\infty} \sum_{s_{1}+s_{2}=n} \sum_{k_{1}, k_{2}=-\infty}^{\infty} e_{4, s_{1}, s_{2}, k_{1}, k_{2}} J_{1}^{s_{1}} J_{2}^{s_{2}} e^{i\left(k_{1} x_{1}+k_{2} x_{2}\right)},(4 \cdot 31)
\end{aligned}
$$

donde los desarrollos en series de Fourier en las expresiones de $x_{1,2}^{\prime}$, contienen tanto el desarrollo de las funciones $\tilde{\mathrm{F}}_{3,4}$ como de $\Omega_{1,2}$. Para simplificar las expresiones, también hemos introducido dentro del desarrollo los parámetros $\varepsilon_{i}$.

\subsubsection{El parámetro de contabilidad}

No todos los términos en un desarrollo de Fourier tienen el mismo tamaño. Por lo tanto, hay términos que tienen una mayor influencia en la dinámica de un sistema que otros. En esta sección se verá cómo ordenar dichos términos en el cálculo de la forma normal.

Es conveniente organizar y separar los términos del mapa por su tamaño relativo. Para clasificar estos términos por su orden de magnitud se introduce un parámetro, llamado parámetro de contabilidad ( [18], [28], [26], [27], [29]), representado por $\lambda$. Este parámetro es esencial para la implementación numérica del método, donde su potencia (la cual es un número natural) representa el orden de magnitud del término. Por otro lado, $\lambda$ tiene un valor numérico de $\lambda=1$, de manera que su introducción no modifica los valores de las diferentes expresiones.

Para la organización de una serie en potencias del parámetro $\lambda$ no existe una regla que sea independiente del mapa al que es aplicado.

Por definición, las acciones utilizadas $J_{i}=y_{i}-y_{i}^{*}$ son cantidades pequeñas. Recordemos que $\left(y_{1}^{*}, y_{2}^{*}\right)$ es el punto central alrededor del cual se va a realizar el desarrollo. Supongamos que dicho punto se encuentra en la capa estocástica de una resonancia de ancho $w \sim \mathcal{O}(\sqrt{\varepsilon})$. Los valores que podrán tomar $y_{1}$ e $y_{2}$ van a estar confinados a un disco de radio $\mathcal{O}(\sqrt{\varepsilon})$. Como $J_{i}$ representa la distancia entre la acción $y_{i}$ y su valor correspondiente del punto central, $J_{i}=y_{i}-y_{i}^{*}$, 
es evidente que $J_{i}$ es también una cantidad de orden $\mathcal{O}(\sqrt{\varepsilon})$. Debido a esto último nosotros establecemos que $J_{i}$ es una cantidad de orden $\lambda$. Luego, en las ecuaciones del mapa hacemos el reemplazo $J_{i} \rightarrow \lambda J_{i}, i=1,2$. Esto hace que, si tenemos un término del desarrollo de Fourier donde $J_{1}$ está elevado a una potencia $s_{1}$ y J $J_{2}$ está elevado a una potencia $s_{2}$, aquél tendrá la forma

$$
\mathrm{J}_{1}^{\mathrm{s}_{1}} \mathrm{~J}_{2}^{\mathrm{s}_{2}} \rightarrow \lambda^{\mathrm{s}_{1}+\mathrm{s}_{2}} \mathrm{~J}_{1}^{\mathrm{s}_{1}} \mathrm{~J}_{2}^{\mathrm{s}_{2}}
$$

y entonces la potencia del parámetro de contabilidad $\lambda$ indicará el orden de magnitud del término.

Por otro lado, para la dependencia de $\lambda$ con los ángulos, es necesario analizar el tamaño de los coeficientes de Fourier, los cuales decaen como $e^{-\sigma|k|}$ ([4I]), con $|k|=\left|k_{1}\right|+\left|k_{2}\right|$, donde $\sigma$ es el radio del dominio de analiticidad de la función que está siendo transformada. Luego, diremos que los términos de tamaño $\mathcal{O}(\sqrt{\varepsilon})$ son de orden $\lambda$, y la condición que queremos que cumplan los términos de Fourier será

$$
\sqrt{\varepsilon}=e^{-\sigma K}
$$

para una cierta constante $\mathrm{K}$, y $\sigma$ calculado para cada función en cuestión. Tomando para $\mathrm{K}$ el entero más cercano a la solución de (4.33), este valor nos indicará cada cuántos órdenes de los términos de Fourier aumentará la potencia de $\lambda$.

Es importante remarcar que esta implementación de $\lambda$ debe ser realizada en la llamada función generatriz ${ }^{6}$ del mapa, a fin de asegurar que la estructura simpléctica del mapa sea mantenida en la expresión final, posterior a la introducción de $\lambda$. Debido a esto, resulta conveniente realizar primero el desarrollo en serie de Fourier de la función generatriz. Una vez hecho esto, y siguiendo el mecanismo desarrollado en esta sección, realizamos la implementación del parámetro de contabilidad $\lambda$. Finalmente, a partir de la expresión de la función

6 Entendemos aquí como función generatriz S de un sistema de 2 grados de libertad (es decir, 4 dimensiones en el espacio de fases) a una función expresada en términos de las 2 acciones nuevas y de los 2 ángulos originales

$$
S \equiv S\left(J_{1}^{\prime}, J_{2}^{\prime}, x_{1}, x_{2}\right)
$$

de manera que, junto con las ecuaciones

$$
\mathrm{J}_{i}=\frac{\partial S}{\partial x_{i}}, \quad x_{i}^{\prime}=\frac{\partial S}{\partial J_{i}^{\prime}}
$$

podemos construir las ecuaciones del mapa en las nuevas variables $\left(\mathrm{J}_{1}^{\prime}, \mathrm{J}_{2}^{\prime}, \mathrm{x}_{1}^{\prime}, \mathrm{x}_{2}^{\prime}\right)$. 
generatriz con $\lambda$ ya implementado, construimos las ecuaciones del mapa al que denominaremos $\mathcal{F}$.

El mapa finalmente tendrá la forma

$$
\begin{aligned}
& J_{1}^{\prime}=J_{1}+\sum_{r=0}^{\infty} \lambda^{r} \sum \mathcal{B}_{1, s_{1}, s_{2}, k_{1}, k_{2}, r} J_{1}^{s_{1}} J_{2}^{s_{2}} e^{i\left(k_{1} x_{1}+k_{2} x_{2}\right)} \\
& J_{2}^{\prime}=J_{2}+\sum_{r=0}^{\infty} \lambda^{r} \sum \mathcal{B}_{2, s_{1}, s_{2}, k_{1}, k_{2}, r} J_{1}^{s_{1}} J_{2}^{s_{2}} e^{i\left(k_{1} x_{1}+k_{2} x_{2}\right)} \\
& x_{1}^{\prime}=x_{1}+\omega_{1}^{*}+\sum_{r=0}^{\infty} \lambda^{r} \sum \mathcal{B}_{3, s_{1}, s_{2}, k_{1}, k_{2}, r} J_{1}^{s_{1}} J_{2}^{s_{2}} e^{i\left(k_{1} x_{1}+k_{2} x_{2}\right)} \\
& x_{2}^{\prime}=x_{2}+\omega_{2}^{*}+\sum_{r=0}^{\infty} \lambda^{r} \sum \mathcal{B}_{4, s_{1}, s_{2}, k_{1}, k_{2}, r} J_{1}^{s_{1}} J_{2}^{s_{2}} e^{i\left(k_{1} x_{1}+k_{2} x_{2}\right)},
\end{aligned}
$$

donde la segunda sumatoria en cada expresión se realiza sobre todos los valores de $s_{1}, s_{2}, k_{1}$ y $k_{2}$ que cumplen que $r_{\text {mín }}(r) \leqslant s_{1}+s_{2}+\left|k_{1}\right|+\left|k_{2}\right| \leqslant r_{\text {máx }}(r)$. Las funciones $r_{\text {mín }}(r)$ y $r_{\text {máx }}(r)$ que determinan los límites de esta suma, debemos calcularlas específicamente para el mapa que estamos estudiando, a partir de los valores de los límites superiores e inferiores que tendrán las variables $s_{1}, s_{2}$, $k_{1}$ y $k_{2}$ para un dado orden $r$ (ver Sección 5.1).

\subsubsection{Obtención de la ecuación de conjugación}

El objetivo de este procedimiento es lograr expresar el mapa $\mathcal{F}$ de manera que resulte más sencillo comprender su dinámica. Este nuevo mapa, llamado forma normal $\mathcal{U}$, es el resultado de una transformación del mapa $\mathcal{F}$ tal que, en las nuevas variables, la forma de éste representa la dinámica de una manera más simple y clara.

Consideremos la transformación canónica que realiza el cambio de variables $\left(J_{1}, J_{2}, x_{1}, x_{2}\right) \rightarrow\left(\Lambda_{1}, \Lambda_{2}, \theta_{1}, \theta_{2}\right)$. Las ecuaciones del mapa en las nuevas variables son

$$
\left\{\begin{array}{l}
\Lambda_{1}^{\prime}=\mathrm{U}_{1}\left(\Lambda_{1}, \Lambda_{2}, \theta_{1}, \theta_{2}\right)=\Lambda_{1}+\tilde{U}_{1}\left(\Lambda_{1}, \Lambda_{2}, \theta_{1}, \theta_{2}\right) \\
\Lambda_{2}^{\prime}=\mathrm{U}_{2}\left(\Lambda_{1}, \Lambda_{2}, \theta_{1}, \theta_{2}\right)=\Lambda_{2}+\tilde{U}_{2}\left(\Lambda_{1}, \Lambda_{2}, \theta_{1}, \theta_{2}\right) \\
\theta_{1}^{\prime}=\mathrm{U}_{3}\left(\Lambda_{1}, \Lambda_{2}, \theta_{1}, \theta_{2}\right)=\theta_{1}+\omega_{1}^{*}+\tilde{u}_{3}\left(\Lambda_{1}, \Lambda_{2}, \theta_{1}, \theta_{2}\right) \\
\theta_{2}^{\prime}=U_{4}\left(\Lambda_{1}, \Lambda_{2}, \theta_{1}, \theta_{2}\right)=\theta_{2}+\omega_{2}^{*}+\tilde{U}_{4}\left(\Lambda_{1}, \Lambda_{2}, \theta_{1}, \theta_{2}\right),
\end{array}\right.
$$


donde $\left(\mathrm{U}_{1}, \mathrm{U}_{2}, \mathrm{U}_{3}, \mathrm{U}_{4}\right)=\mathbf{U}$ y $\left(\tilde{\mathrm{U}}_{1}, \tilde{\mathrm{U}}_{2}, \tilde{\mathrm{U}}_{3}, \tilde{\mathrm{U}}_{4}\right)=\tilde{\mathbf{U}}$. Este mapa, al igual que el de la Ec. (4.24), también puede ser expresado como un desarrollo en serie de Fourier,

$$
\begin{aligned}
& \Lambda_{1}^{\prime}=\Lambda_{1}+\sum_{r=1}^{\infty} \lambda^{r} \sum \mathcal{W}_{1, s_{1}, s_{2}, \tilde{k}_{1}, \tilde{k}_{2}, r}^{(r)} \Lambda_{1}^{s_{1}} \Lambda_{2}^{s_{2}} e^{i\left(\tilde{k}_{1} \theta_{1}+\tilde{k}_{2} \theta_{2}\right)} \\
& \Lambda_{2}^{\prime}=\Lambda_{2}+\sum_{r=1}^{\infty} \lambda^{r} \sum \mathcal{W}_{2, s_{1}, s_{2}, \tilde{k}_{1}, \tilde{k}_{2}, r}^{(r)} \Lambda_{1}^{s_{1}} \Lambda_{2}^{s_{2}} e^{i\left(\tilde{k}_{1} \theta_{1}+\tilde{k}_{2} \theta_{2}\right)} \\
& \theta_{1}^{\prime}=\theta_{1}+\omega_{1}^{*}+\sum_{r=1}^{\infty} \lambda^{r} \sum \mathcal{W}_{3, s_{1}, s_{2}, \tilde{k}_{1}, \tilde{k}_{2}, r}^{(r} \Lambda_{1}^{s_{1}} \Lambda_{2}^{s_{2}} e^{i\left(\tilde{k}_{1} \theta_{1}+\tilde{k}_{2} \theta_{2}\right)} \\
& \theta_{2}^{\prime}=\theta_{2}+\omega_{2}^{*}+\sum_{r=1}^{\infty} \lambda^{r} \sum \mathcal{W}_{4, s_{1}, s_{2}, \tilde{k}_{1}, \tilde{k}_{2}, r}^{(r)} \Lambda_{1}^{s_{1}} \Lambda_{2}^{s_{2}} e^{i\left(\tilde{k}_{1} \theta_{1}+\tilde{k}_{2} \theta_{2}\right)},
\end{aligned}
$$

donde la segunda sumatoria posee como límites inferior y superior las cantidades $l_{\text {mín }}\left(s_{1}, s_{2}, \tilde{k}_{1}, \tilde{k}_{2}, r\right)$ y $l_{\text {máx }}\left(s_{1}, s_{2}, \tilde{k}_{1}, \tilde{k}_{2}, r\right)$, que dependerán de los límites inferiores y superiores de los índices $s_{1}, s_{2}, \tilde{k}_{1}, \tilde{k}_{2}$ y $r$, como sucede en la Ec. (4.34) para $r_{\text {mín }}(r)$ y $r_{\text {máx }}(r)$. Este nuevo mapa, al igual que el original, tiene la forma de un mapa cuasi torsional (Ec. (4.24)).

En cuanto a los índices $\tilde{k}_{1}$ y $\tilde{k}_{2}$, éstos no serán valores arbitrarios, sino que pertenecerán a un conjunto llamado módulo resonante $\mathcal{K}$ ([18], [26], [29]), formado por todos los vectores $\mathbf{k} \in \mathbb{Z}^{2}$ no nulos que satisfacen la Ec. (4.21), es decir, que son paralelos al vector $\mathbf{k}_{\mathrm{r}}$,

$$
\mathcal{K}=\left\{\mathbf{k} \in \mathbb{Z}^{2} / \mathbf{k}=\alpha \mathbf{k}_{r}, \alpha \neq 0\right\},
$$

donde $\alpha \in \mathbb{Z}-\{0\}$ es una constante. Este conjunto puede interpretarse como el conjunto de todos los armónicos de la resonancia que se está estudiando.

Necesitamos entonces encontrar un conjunto de transformaciones $\Phi_{i}, i=$ $1, \ldots, 4$ (que deben ser simplécticas para que preserven el volumen) que transformen el mapa $\mathcal{F}$ representado por el conjunto de variables $\left(J_{1}, J_{2}, x_{1}, x_{2}\right)$ en un nuevo mapa $\mathcal{U}$, expresado en el nuevo conjunto de variables transformadas $\left(\Lambda_{1}, \Lambda_{2}, \theta_{1}, \theta_{2}\right)$. Esta transformación, por construcción, expresa las variables originales como funciones de las nuevas variables

$$
\begin{aligned}
& \mathrm{J}_{1}=\Phi_{1}\left(\Lambda_{1}, \Lambda_{2}, \theta_{1}, \theta_{2}\right) \\
& \mathrm{J}_{2}=\Phi_{2}\left(\Lambda_{1}, \Lambda_{2}, \theta_{1}, \theta_{2}\right) \\
& x_{1}=\Phi_{3}\left(\Lambda_{1}, \Lambda_{2}, \theta_{1}, \theta_{2}\right) \\
& x_{2}=\Phi_{4}\left(\Lambda_{1}, \Lambda_{2}, \theta_{1}, \theta_{2}\right) .
\end{aligned}
$$


Según la Ec. (4.4.3) vemos que el nuevo mapa $U$ es cercano a la identidad. Por ende, las transformaciones $\Phi_{i}$ deberán ser también cercanas a la identidad, expresadas de la forma

$$
\left\{\begin{array}{l}
\Phi_{1}\left(\Lambda_{1}, \Lambda_{2}, \theta_{1}, \theta_{2}\right)=\Lambda_{1}+\sum_{j=1}^{\infty} \Phi_{1 j}\left(\Lambda_{1}, \Lambda_{2}, \theta_{1}, \theta_{2}\right) \\
\Phi_{2}\left(\Lambda_{1}, \Lambda_{2}, \theta_{1}, \theta_{2}\right)=\Lambda_{2}+\sum_{j=1}^{\infty} \Phi_{2 j}\left(\Lambda_{1}, \Lambda_{2}, \theta_{1}, \theta_{2}\right) \\
\Phi_{3}\left(\Lambda_{1}, \Lambda_{2}, \theta_{1}, \theta_{2}\right)=\theta_{1}+\sum_{j=1}^{\infty} \Phi_{3 j}\left(\Lambda_{1}, \Lambda_{2}, \theta_{1}, \theta_{2}\right) \\
\Phi_{4}\left(\Lambda_{1}, \Lambda_{2}, \theta_{1}, \theta_{2}\right)=\theta_{2}+\sum_{j=1}^{\infty} \Phi_{4 j}\left(\Lambda_{1}, \Lambda_{2}, \theta_{1}, \theta_{2}\right)
\end{array}\right.
$$

donde $\Phi_{i j}$ son funciones pequeñas de orden $\mathcal{O}(j)$ en potencias de $\lambda$. Estas funciones también pueden ser escritas como series de Fourier, y agrupadas según potencias de $\lambda$

$$
\left\{\begin{array}{l}
\Phi_{1}\left(\Lambda_{1}, \Lambda_{2}, \theta_{1}, \theta_{2}\right)=\Lambda_{1}+\sum_{j=0}^{\infty} \lambda^{j} \sum v_{1, s_{1}, s_{2}, k_{1}, k_{2}}^{(j)} \Lambda_{1}^{s_{1}} \Lambda_{2}^{s_{2}} e^{i\left(k_{1} \theta_{1}+k_{2} \theta_{2}\right)} \\
\Phi_{2}\left(\Lambda_{1}, \Lambda_{2}, \theta_{1}, \theta_{2}\right)=\Lambda_{2}+\sum_{j=0}^{\infty} \lambda^{j} \sum v_{2, s_{1}, s_{2}, k_{1}, k_{2}}^{(j)} \Lambda_{1}^{s_{1}} \Lambda_{2}^{s_{2}} e^{i\left(k_{1} \theta_{1}+k_{2} \theta_{2}\right)} \\
\Phi_{3}\left(\Lambda_{1}, \Lambda_{2}, \theta_{1}, \theta_{2}\right)=\theta_{1}+\sum_{j=0}^{\infty} \lambda^{j} \sum v_{3, s_{1}, s_{2}, k_{1}, k_{2}}^{(j)} \Lambda_{1}^{s_{1}} \Lambda_{2}^{s_{2}} e^{i\left(k_{1} \theta_{1}+k_{2} \theta_{2}\right)} \\
\Phi_{4}\left(\Lambda_{1}, \Lambda_{2}, \theta_{1}, \theta_{2}\right)=\theta_{2}+\sum_{j=0}^{\infty} \lambda^{j} \sum v_{4, s_{1}, s_{2}, k_{1}, k_{2}}^{(j)} \Lambda_{1}^{s_{1}} \Lambda_{2}^{s_{2}} e^{i\left(k_{1} \theta_{1}+k_{2} \theta_{2}\right)}
\end{array}\right.
$$

donde la segunda sumatoria se realiza sobre todos los valores de $s_{1}, s_{2}, k_{1}$ y $k_{2}$ que cumplen la condición $0 \leqslant s_{1}+s_{2}+\left|k_{1}\right|+\left|k_{2}\right| \leqslant r_{\text {máx }}(\mathfrak{j})$, al igual que para el mapa $\mathcal{F}$.

La transformación de la Ec. (4.40) se encuentra mediante la resolución de la llamada ecuación de conjugación ([5], [30], [75]). Veamos cómo construir esta ecuación.

Es posible expresar la variable $J_{i}^{\prime}$ a partir de la Ec. (4.38), escribiendo cada variable primada, como $J_{i}^{\prime}=\Phi_{i}\left(\Lambda_{1}^{\prime}, \Lambda_{2}^{\prime}, \theta_{1}^{\prime}, \theta_{2}^{\prime}\right)$. Luego, reemplazando las variables $\left(\Lambda_{1}^{\prime}, \Lambda_{2}^{\prime}, \theta_{1}^{\prime}, \theta_{2}^{\prime}\right)$ por las correspondientes expresiones de la Ec. (4.35), obtenemos

$$
\mathrm{J}_{i}^{\prime}=\Phi_{i}\left(\mathrm{U}_{1}\left(\mathbf{v}_{\mathfrak{u}}\right), \mathrm{U}_{2}\left(\mathbf{v}_{\mathfrak{u}}\right), \mathrm{U}_{3}\left(\mathbf{v}_{\mathfrak{u}}\right), \mathrm{U}_{4}\left(\mathbf{v}_{\mathfrak{u}}\right)\right),
$$

donde $\mathbf{v}_{\mathfrak{u}}=\left(\Lambda_{1}, \Lambda_{2}, \theta_{1}, \theta_{2}\right)$. De la misma manera es posible obtener ecuaciones análogas a la Ec. (4.41) para las variables $\chi_{i}^{\prime}, i=1,2$. El miembro derecho de 
la Ec. (4.41) junto con el de las ecuaciones correspondientes para $x_{i}^{\prime}$ se pueden reescribir en forma compacta como $\Phi_{i}\left(\mathbf{U}\left(\mathbf{v}_{\mathcal{U}}\right)\right)$ o bien $\left(\Phi_{i} \circ \mathbf{U}\right)\left(\mathbf{v}_{\mathcal{U}}\right)$.

Si miramos ahora las ecuaciones del mapa de la Ec. (4.24), haciendo el reemplazo de la Ec. (4.28), es posible escribir a $J_{i}^{\prime}$ como $J_{i}^{\prime}=F_{i}\left(J_{1}, J_{2}, x_{1}, x_{2}\right)$. A través de la Ec. (4.38) podemos expresar a $J_{i}^{\prime}$ como función de las nuevas variables. Haciendo esto, la expresión que se obtiene para $J_{i}^{\prime}$ es

$$
\mathrm{J}_{\mathfrak{i}}^{\prime}=\mathrm{F}_{\mathfrak{i}}\left(\Phi_{1}\left(\mathbf{v}_{\mathfrak{U}}\right), \Phi_{2}\left(\mathbf{v}_{\mathfrak{U}}\right), \Phi_{3}\left(\mathbf{v}_{\mathfrak{U}}\right), \Phi_{4}\left(\mathbf{v}_{\mathfrak{U}}\right)\right) .
$$

De la misma manera que hicimos con la Ec. (4.41), se puede reescribir la Ec. (4.42) como $J_{i}=F_{i}\left(\boldsymbol{\Phi}\left(\mathbf{v}_{u}\right)\right)$, o bien $\left(F_{i} \circ \boldsymbol{\Phi}\right)\left(\mathbf{v}_{u}\right)$, con $\boldsymbol{\Phi}=\left(\Phi_{1}, \Phi_{2}, \Phi_{3}, \Phi_{4}\right)$. Igualando las Ecs. (4.41) y (4.42) obtenemos

$$
\left(F_{i} \circ \Phi\right)\left(\Lambda_{1}, \Lambda_{2}, \theta_{1}, \theta_{2}\right)=\left(\Phi_{i} \circ \mathbf{U}\right)\left(\Lambda_{1}, \Lambda_{2}, \theta_{1}, \theta_{2}\right) .
$$

Esta ecuación se conoce como ecuación de conjugación. Su resolución se lleva a cabo mediante la implementación de un algoritmo iterativo, que veremos en la Sección 4.4.4.

\subsubsection{Construcción y resolución de la ecuación homológica}

Consideremos, para las funciones $F_{i}, U_{i}$ y $\Phi_{i}$ de la Ec. (4.43), con $i=1, \ldots, 4$, las sumas sobre $s_{1}, s_{2}, k_{1}$ y $k_{2}$ para un dado orden $j, y$ llamemos al resultado $F_{i}^{(j)}, u_{i}^{(j)}$ y $\Phi_{i}^{(j)}$, respectivamente. Esto nos permite tener los términos agrupados según el orden de $\lambda$, y condensados en un sólo término por orden. De esta manera, las expresiones del mapa $\mathcal{F}$, la forma normal $U$ y la transformación $\boldsymbol{\Phi}$ pueden ser expresadas como

$$
\begin{aligned}
F_{i} & =F_{i}{ }^{(0)}+\lambda F_{i}{ }^{(1)}+\lambda^{2} F_{i}{ }^{(2)}+\cdots=\sum_{j=0}^{\infty} \lambda^{j} F_{i}^{(j)} \\
u_{i} & =u_{i}{ }^{(0)}+\lambda u_{i}{ }^{(1)}+\lambda^{2} u_{i}{ }^{(2)}+\cdots=\sum_{j=0}^{\infty} \lambda^{j} u_{i}^{(j)} \\
\Phi_{i} & =\Phi_{i}{ }^{(0)}+\lambda \Phi_{i}{ }^{(1)}+\lambda^{2} \Phi_{i}{ }^{(2)}+\cdots=\sum_{j=0}^{\infty} \lambda^{j} \Phi_{i}^{(j)},
\end{aligned}
$$

donde el supraíndice $j$ indica el orden del término en potencias de $\lambda$. 
Reemplazando las expresiones para $F_{i}$ y $\Phi_{i}$ de las Ecs. (4.44) en el miembro izquierdo de la Ec. (4.43) obtenemos 7

$$
\left(F_{i} \circ \boldsymbol{\Phi}\right)\left(\mathbf{v}_{\mathcal{U}}\right)=\left(\mathrm{F}_{i}^{(0)}+\lambda \mathrm{F}_{i}^{(1)}+\ldots\right) \circ\left(\Phi^{(0)}+\lambda \Phi^{(1)}+\ldots\right)\left(\mathbf{v}_{\mathfrak{U}}\right) .
$$

De una manera análoga podemos reescribir el miembro derecho de la Ec. (4.43) en la forma:

$$
\left(\Phi_{i} \circ \mathbf{U}\right)\left(\mathbf{v}_{\mathcal{U}}\right)=\left(\Phi_{i}^{(0)}+\lambda \Phi_{i}^{(1)}+\ldots\right) \circ\left(\mathbf{U}^{(0)}+\lambda \mathbf{U}^{(1)}+\ldots\right)\left(\mathbf{v}_{\mathfrak{U}}\right) .
$$

Existen algunos términos de las Ecs. (4.45) y (4.46) cuyas expresiones pueden calcularse trivialmente, y se detallan a continuación:

- Dado que $\mathbf{U}^{(0)}=\left(\Lambda_{1}, \Lambda_{2}, \theta_{1}+\omega_{1}^{*}, \theta_{2}+\omega_{2}^{*}\right)$, luego se obtiene $\left(\Phi_{i}^{(\mathrm{N})} \circ\right.$ $\left.\mathbf{U}^{(0)}\right)=\Phi_{i}^{(\mathrm{N})}\left(\Lambda_{1}, \Lambda_{2}, \theta_{1}+\omega_{1}^{*}, \theta_{2}+\omega_{2}^{*}\right)$.

- Por otro lado, de la composición del término de orden $\mathcal{O}(0)$ de $F_{i}$ con los de orden $\mathcal{O}(\mathrm{N})$ de $\boldsymbol{\Phi}$ obtenemos $\left(\mathrm{F}_{i}\left({ }^{(0)} \circ \boldsymbol{\Phi}^{(\mathrm{N})}\right)=\Phi_{i}^{(\mathrm{N})}\left(\Lambda_{1}, \Lambda_{2}, \theta_{1}, \theta_{2}\right)\right.$.

- Por último, mediante un razonamiento similar al anterior, de la composición $\left(\Phi_{i}{ }^{(0)} \circ \mathbf{U}^{(N)}\right)$ se obtiene $\left(\Phi_{i}{ }^{(0)} \circ \mathbf{U}^{(N)}\right)=U_{i}^{(N)}\left(\Lambda_{1}, \Lambda_{2}, \theta_{1}, \theta_{2}\right)$.

Al resto de los términos de orden $\mathrm{N}$, cuya composición no es trivial, los vamos a agrupar según

$$
\mathrm{Q}_{i}^{(\mathrm{N})}=\left[\sum_{k=1}^{\mathrm{N}} \mathrm{F}_{i}{ }^{(k)}\left([\boldsymbol{\Phi}]_{\leqslant N-1}\right)-\sum_{k=1}^{\mathrm{N}-1} \Phi_{i}{ }^{(k)}\left([\mathbf{U}]_{\leqslant N-1}\right)\right]_{N},
$$

donde hemos utilizado la notación [30]:

$$
[\mathrm{f}]_{\leqslant \mathrm{N}-1} \equiv \mathrm{f}_{1}+\mathrm{f}_{2}+\cdots+\mathrm{f}_{\mathrm{N}-1}, \quad[\mathrm{f}]_{\mathrm{N}} \equiv \mathrm{f}_{\mathrm{N}},
$$

siendo $f_{i}$ términos de orden $\mathcal{O}(i)$ en $\lambda$ de un desarrollo en serie de Fourier para alguna función compleja $f$.

7 Recordemos que la composición de la función $F_{i}$ con el vector $\Phi$ se entiende como la composición de $F_{i}$ con las componentes de $\Phi$, es decir

$$
F_{i} \circ \boldsymbol{\Phi}=F_{i}\left(\Phi_{1}, \Phi_{2}, \Phi_{3}, \Phi_{4}\right)=F_{i}\left(\sum_{j=0}^{\infty} \Phi_{1}{ }^{(j)}, \sum_{j=0}^{\infty} \Phi_{2}{ }^{(j)}, \sum_{j=0}^{\infty} \Phi_{3}{ }^{(j)}, \sum_{j=0}^{\infty} \Phi_{4}{ }^{(j)}\right),
$$

donde $\Phi_{i}^{(j)}=\Phi_{i}^{(j)}\left(\Lambda_{1}, \Lambda_{2}, \theta_{1}, \theta_{2}\right)$. La composición $\Phi_{i} \circ \mathbf{U}$ es análoga. 
Reagrupando todas las composiciones que llamamos triviales en el miembro izquierdo y el resto (reunidas en $\mathrm{Q}_{i}^{(\mathrm{N})}$ ) en el miembro derecho, la ecuación de conjugación para un orden $\mathcal{O}(\mathrm{N})$ tendrá la forma

$$
\Phi_{i}^{(\mathrm{N})}\left(\mathbf{v}_{\mathfrak{U}}^{\prime}\right)-\Phi_{i}^{(\mathrm{N})}\left(\mathbf{v}_{\mathcal{U}}\right)+\mathrm{U}_{i}^{(\mathrm{N})}\left(\mathbf{v}_{\mathcal{U}}\right)=\mathrm{Q}_{\mathfrak{i}}^{(\mathrm{N})}\left(\mathbf{v}_{\mathcal{U}}\right) .
$$

donde $\mathbf{v}_{u}^{\prime}=\left(\Lambda_{1}, \Lambda_{2}, \theta_{1}+\omega_{1}^{*}, \theta_{2}+\omega_{2}^{*}\right)$. La Ec. (4.49) es la llamada ecuación homológica. Siguiendo el desarrollo realizado en esta sección, podemos ver que la resolución de la ecuación de conjugación (Ec. (4.43)) es equivalente a la resolución de la ecuación homológica (Ec. (4-49)). Veremos a continuación cómo resolver esta ecuación.

Introducimos el operador lineal de diferencias de una cierta función $f$

$$
\Delta_{\omega} f\left(\mathbf{v}_{\mathcal{U}}\right)=f\left(\mathbf{v}_{\mathcal{U}}^{\prime}\right)-f\left(\mathbf{v}_{\mathcal{U}}\right)
$$

y lo aplicamos a los términos de $\Phi_{i}^{(\mathrm{N})}$ del miembro izquierdo de la Ec. (4.49), obteniendo

$$
\Delta_{\omega} \Phi_{i}^{(\mathrm{N})}\left(\mathbf{v}_{\mathcal{U}}\right)+\mathrm{U}_{\mathfrak{i}}^{(\mathrm{N})}\left(\mathbf{v}_{\mathcal{U}}\right)=\mathrm{Q}_{\mathfrak{i}}^{(\mathrm{N})}\left(\mathbf{v}_{\mathcal{U}}\right)
$$

Dado que las expresiones de las funciones involucradas en los desarrollos tanto de $\Phi_{i}$ como de $U_{i}$ son series de Fourier, veamos cómo afecta este operador a un término de la forma

$$
\Gamma \Lambda_{1}^{s_{1}} \Lambda_{2}^{s_{2}} e^{i\left(k_{1} \theta_{1}+k_{2} \theta_{2}\right)} .
$$

Aplicando el operador de diferencias sobre este término obtenemos

$$
\begin{aligned}
& \Delta_{\omega}\left(\Gamma \Lambda_{1}^{s_{1}} \Lambda_{2}^{s_{2}} e^{i\left(k_{1} \theta_{1}+k_{2} \theta_{2}\right)}\right)= \\
& \Gamma \Lambda_{1}^{s_{1}} \Lambda_{2}^{s_{2}} e^{i\left(k_{1}\left(\theta_{1}+\omega_{1}^{*}\right)+k_{2}\left(\theta_{2}+\omega_{2}^{*}\right)\right)}-\Gamma \Lambda_{1}^{s_{1}} \Lambda_{2}^{s_{2}} e^{i\left(k_{1} \theta_{1}+k_{2} \theta_{2}\right)}= \\
& \Gamma \Lambda_{1}^{s_{1}} \Lambda_{2}^{s_{2}} e^{i\left(k_{1} \theta_{1}+k_{2} \theta_{2}\right)}\left(e^{i\left(k_{1} \omega_{1}^{*}+k_{2} \omega_{2}^{*}\right)}-1\right) .
\end{aligned}
$$

Como mencionamos en la Sección 4·4·3, los términos que constituyen las funciones $U_{i}\left(\mathbf{v}_{\mathcal{U}}\right)$ son aquellos que pertenecen al módulo resonante $\mathcal{K}$ (Ec. (4.37)). Por ende, si la Ec. (4.53) está siendo aplicada a un término de $U_{i}$, tendremos que $k_{1} \omega_{1}^{*}+k_{2} \omega_{2}^{*}=0$, y luego

$$
\Delta_{\omega}\left(\Gamma \Lambda_{1}^{s_{1}} \Lambda_{2}^{s_{2}} e^{i\left(k_{1} \theta_{1}+k_{2} \theta_{2}\right)}\right)=0
$$


Esto nos indica que los términos que integran $\mathrm{U}_{i}^{(\mathrm{N})}$ forman el espacio nulo, o núcleo, del operador $\Delta_{\omega}$

$$
\begin{aligned}
\mathcal{N}\left(\Delta_{\omega}\right)= & \left\{\mathcal{W}_{s_{1}, s_{2}, k_{1}, k_{2}}^{(\mathrm{N})} \Lambda_{1}^{s_{1}} \Lambda_{2}^{s_{2}} e^{i\left(k_{1} \theta_{1}+k_{2} \theta_{2}\right)}\right. \\
& \left.\mathcal{W}^{(\mathrm{N})} \in \mathbb{C}, s_{1}, s_{2} \in \mathbb{N}, k_{1}, k_{2} \in \mathbb{Z}, k_{1} \omega_{1}^{*}+k_{2} \omega_{2}^{*}=0\right\} \cdot(4 \cdot 55)
\end{aligned}
$$

Viendo la Ec. (4.51) podemos inferir que un subconjunto de términos de $Q_{i}^{(N)}$ se corresponderán con $U_{i}^{(N)}$ y el subconjunto complementario con el operador $\Delta_{\omega} \Phi_{i}^{(\mathrm{N})}$. Luego, a partir de la Ec. (4.55), vemos que los términos de $\mathrm{Q}_{i}^{(\mathrm{N})}$ que satisfacen la condición $Q_{i}^{(N)} \in \mathcal{N}$ son los que consituyen $U_{i}^{(N)}$, mientras que el resto de los términos estarán asociados a $\Delta_{\omega} \Phi_{i}^{(N)}$. Para estos términos tenemos que los valores $\left(k_{1}, k_{2}\right)$ no pertenecerán al módulo resonante $\mathcal{K}$, esto es $k_{1} \omega_{1}^{*}+k_{2} \omega_{2}^{*} \neq 0$.

Si aplicamos la Ec. (4.53) sobre un término de $\Phi_{i}^{(\mathrm{N})}$ obtenemos

$$
\begin{aligned}
& \Delta_{\omega}\left(\nu \Lambda_{1}^{s_{1}} \Lambda_{2}^{s_{2}} e^{i\left(k_{1} \theta_{1}+k_{2} \theta_{2}\right)}\right)= \\
& \nu \Lambda_{1}^{s_{1}} \Lambda_{2}^{s_{2}} e^{i\left(k_{1} \theta_{1}+k_{2} \theta_{2}\right)}\left(e^{i\left(k_{1} \omega_{1}^{*}+k_{2} \omega_{2}^{*}\right)}-1\right) \neq 0,
\end{aligned}
$$

donde, para simplificar la notación, hemos omitido los subíndices $s_{1}, s_{2}, k_{1}, k_{2}$ en la expresión del coeficiente $\mathcal{V}$.

Concluimos entonces que los términos de $\Phi_{i}^{(\mathrm{N})}$ son autovectores del operador $\Delta_{\omega}$ con autovalor $e^{i\left(k_{1} \omega_{1}^{*}+k_{2} \omega_{2}^{*}\right)}-1$, y formarán la imagen de $\Delta_{\omega}$

$$
\begin{aligned}
\mathcal{R}\left(\Delta_{\omega}\right)= & \left\{\mathcal{V}_{\mathrm{s}_{1}, \mathrm{~s}_{2}, \mathrm{k}_{1}, \mathrm{k}_{2}}^{(\mathrm{N})} \Lambda_{1}^{s_{1}} \Lambda_{2}^{s_{2}} e^{i\left(k_{1} \theta_{1}+k_{2} \theta_{2}\right)},\right. \\
& \left.\mathcal{V}^{(\mathrm{N})} \in \mathbb{C}, s_{1}, s_{2} \in \mathbb{N}, \mathrm{k}_{1}, \mathrm{k}_{2} \in \mathbb{Z}, \mathrm{k}_{1} \omega_{1}^{*}+\mathrm{k}_{2} \omega_{2}^{*} \neq 0\right\} .
\end{aligned}
$$

De la Ec. (4.56) concluimos también que el operador $\Delta_{\omega}$ es invertible en el subespacio $\mathcal{R}$. De esta manera podemos obtener los términos que forman parte de la transformación $\Phi_{i}$ y de la forma normal $U_{i}$, con $i=1, \ldots, 4$, como sigue.

Empleando las definiciones de núcleo e imagen de $\Delta_{\omega}$ (Ecs. (4.55) y (4.57), respectivamente), obtenemos como solución para la Ec. (4.49)

$$
\begin{aligned}
& \mathrm{U}_{i}^{(\mathrm{N})}=\text { términos del núcleo de } \Delta_{\omega} \text { con respecto a } \mathrm{Q}_{i}^{(\mathrm{N})}, \\
& \Phi_{i}^{(\mathrm{N})}=\Delta_{\omega}{ }^{-1}\left(\mathrm{Q}_{i}^{(\mathrm{N})}-\mathrm{U}_{i}^{(\mathrm{N})}\right)+\mathcal{A}_{i}{ }^{(\mathrm{N})},
\end{aligned}
$$

donde $\mathcal{A}_{i}{ }^{(\mathrm{N})}$ está formado por términos arbitrarios del núcleo de $\Delta_{\omega}$, de órdenes menor o igual a $\mathrm{N}$. La inclusión de $\mathcal{A}_{i}{ }^{(\mathrm{N})}$ en la definición de $\Phi_{i}^{(\mathrm{N})}$ es 
debida a que, si aplicamos el operador de diferencias a la segunda igualdad de la Ec. (4.58), obtenemos

$$
\Delta_{\omega} \Phi_{i}^{(\mathrm{N})}=\mathrm{Q}_{i}^{(\mathrm{N})}-\mathrm{U}_{i}^{(\mathrm{N})}+\Delta_{\omega} \mathcal{A}_{i}^{(\mathrm{N})},
$$

y, dado que $\mathcal{A}_{i}{ }^{(\mathrm{N})}$ está formado por términos del núcleo de $\Delta_{\omega}$, tenemos que $\Delta_{\omega} \mathcal{A}_{i}{ }^{(\mathrm{N})}=0$, y la Ec. (4.59) resulta ser la Ec. (4.51).

Una vez definidas las formas de los términos que constituyen $U_{i}^{(N)}$ y a $\Phi_{i}^{(N)}$, la resolución de la Ec. (4.49) es inmediata. El algoritmo iterativo utilizado en su resolución es el siguiente. Para un dado orden $N$ (comenzando con $N=1$ ) tomamos, uno a uno, todos los términos de $Q_{i}^{(N)}$ y comprobamos si pertenece al núcleo o a la imagen de $\Delta_{\omega}$, analizando si satisface la condición de resonancia $k_{1} \omega_{1}^{*}+k_{2} \omega_{2}^{*}=0$. Si esta condición se cumple, el término tiene la forma de un elemento del núcleo $\mathcal{N} y$, por lo tanto, formará parte de $\mathrm{U}_{i}^{(\mathrm{N})}$. Si la condición no es satisfecha, el término pertenecerá a la transformación $\Phi_{i}^{(\mathrm{N})}$. Recordemos que, viendo la Ec. (4.53), deberemos dividir el término por $e^{i\left(k_{1} \omega_{1}^{*}+k_{2} \omega_{2}^{*}\right)}-1$, para que éste tenga la forma de un elemento de $\mathcal{R}$. A partir de la Ec. (4.53) podemos ver que la forma de los términos que pertenecen a $\Phi_{i}^{(\mathrm{N})}$ son

$$
\frac{\Gamma}{e^{i\left(k_{1} \omega_{1}^{*}+k_{2} \omega_{2}^{*}\right)}-1} \Lambda_{1}^{s_{1}} \Lambda_{2}^{s_{2}} e^{i\left(k_{1} \theta_{1}+k_{2} \theta_{2}\right)} .
$$

A partir de la Ec. (4.60) podemos hacer un desarrollo en series de Taylor del denominador, obteniendo

$$
e^{i\left(k_{1} \omega_{1}^{*}+k_{2} \omega_{2}^{*}\right)}-1 \approx 1+k_{1} \omega_{1}^{*}+k_{2} \omega_{2}^{*}+\cdots-1=k_{1} \omega_{1}^{*}+k_{2} \omega_{2}^{*}+\mathcal{O}(2) .
$$

Dado que $\omega_{1}^{*}=\omega_{2}^{*}=\omega$, llegamos a que el término de más bajo orden del desarrollo es de la forma $\left(k_{1}+k_{2}\right) \omega$.

Como la resonancia que estudiaremos es la $1: 1$, el valor no nulo más pequeño para $k_{1}+k_{2}$ es 1 . Esto sucede cuando $k_{2}=-k_{1}+1$, y ocurre cuando estamos cerca de la condición de resonancia, o de alguno de sus armónicos. Esta condición hace que el tamaño del denominador esté acotado a $\omega$. Sin embargo, es posible mostrar (aunque no lo haremos en este trabajo) que existe en el numerador un factor de la forma $k_{1}-k_{2}=2 k_{1}-1$ donde, a medida que contemplamos armónicos mayores, crece el valor de $k_{1}$, haciendo que este factor también crezca. Si este factor lo reescribimos en el denominador, dividiendo a la cantidad $k_{1}+k_{2}$ obtenemos un denominador que se hará cada vez más pequeño, al considerar los pares $\left(k_{1}, k_{2}\right)$ cercanos a los armónicos de la resonacia $1: 1$. 
Estos términos contribuirán a un fenómeno conocido como acumulación de denominadores, que explicaremos brevemente a continuación. Para la construcción de la transformación a orden $\mathrm{N}$, cuando se hallan los términos pertenecientes a $\Phi_{i}^{(\mathrm{N})}$, éstos términos contendrán un denominador con la forma $\left(k_{1}+k_{2}\right) \omega /\left(k_{1}-k_{2}\right)$. Al construir la tranformación de orden $N+1$, este término aparecerá en la ecuación homológica (Ec. (4.47)). Luego, al construir los términos que formarán parte de $\Phi_{i}^{(\mathrm{N}+1)}$ aparecerá este término, dividido por un nuevo factor $k_{1}-k_{2}$, con $k_{1} y k_{2}$ distintos a los hallados para orden N. Por lo tanto, para cada orden de la transformación, los términos de $\Phi_{i}$ van acumulando cada vez más denominadores, haciendo que los coeficientes de dichos términos comiencen a aumentar a partir de un dado orden. Esto provoca que el error en la tranformación aumente, como vereemos más adelante.

\subsubsection{Comprobación de simplecticidad}

Para que la transformación a formas normales $\boldsymbol{\Phi}$ hallada en la Sección 4·4·4 sea canónica, es necesario que sea simpléctica.

Una vez obtenida la solución de la ecuación homológica en el paso $\mathrm{N}$, obtendremos la transformación hasta este orden, $\Phi_{i}^{(N)}$. Antes de continuar con la resolución de la ecuación homológica para el orden $\mathcal{O}(\mathrm{N}+1)$, debemos asegurarnos que la transformación hasta aquí hallada es simpléctica, al menos hasta orden $\mathcal{O}(\mathrm{N})$. Esto se lleva a cabo mediante el cálculo de todos los corchetes de Poisson. La condición de simplecticidad exige que los corchetes calculados entre la transformación asociada a una acción $\mathrm{J}_{i} \mathrm{y}$ la asociada a su ángulo conjugado $x_{i}$ (es decir, $\left\{\Phi_{x_{i}}, \Phi_{J_{i}}\right\}$, con $i=1,2$ ) valgan 1 , mientras que el resto de los corchetes obtenidos mediante la combinación entre las transformaciones, es $\operatorname{decir}\left\{\Phi_{x_{i}}, \Phi_{J_{j}}\right\},\left\{\Phi_{x_{i}}, \Phi_{x_{j}}\right\}$ y $\left\{\Phi_{\mathrm{J}_{i}}, \Phi_{\mathrm{J}_{j}}\right\}$, con $i \neq j$, deberán valer 0.

Como aquí estamos calculando una transformación truncada a orden $\mathcal{O}(\mathrm{N})$, en general, la condición de simplecticidad no será satisfecha de forma exacta. Esto es, dado el orden $\mathcal{O}(\mathrm{N})$ de la transformación, solamente podremos exigir que ésta cumpla la condición de ser "simpléctica hasta orden $\mathcal{O}(\mathrm{N})$ "(de aquí en adelante consideraremos que una función es simpléctica si cumple la condición de simplecticidad hasta orden $\mathcal{O}(\mathrm{N})$ ). Esta nueva condición exige que los corchetes cumplan

$$
\begin{gathered}
\left\{\Phi_{x_{i}}, \Phi_{J_{i}}\right\}=1+\mathcal{O}(N+1), \quad i=1,2 \\
\left\{\Phi_{x_{i}}, \Phi_{J_{j}}\right\}=\left\{\Phi_{x_{i}}, \Phi_{x_{j}}\right\}=\left\{\Phi_{J_{i}}, \Phi_{J_{j}}\right\}=\mathcal{O}(N+1), \text { con } i \neq j .
\end{gathered}
$$

Puede ocurrir que, luego de analizar las condiciones de simplecticidad, las expresiones de la Ec. (4.6I) no se satisfagan y aparezcan términos no nulos 
de orden $\leqslant N$, haciendo que la transformación hallada no sea simpléctica. Entonces, es necesario emplear alguna técnica para lograr que la transformación resulte simpléctica sin alterar la forma de ésta ni de las ecuaciones de la forma normal $\mathbf{U}^{(\mathrm{N})}$.

De acuerdo con la Ec. (4.58), la transformación $\Phi_{i}^{(\mathrm{N})}$ está compuesta por dos conjuntos de términos. El primer conjunto de términos forma parte de la imagen $\mathcal{R}$ del operador $\Delta_{\omega}$, mientras que el segundo grupo está compuesto por términos arbitrarios del núcleo con respecto al operador $\Delta_{\omega}$. Para lograr que $\Phi_{i}^{(\mathrm{N})}$ cumpla la condición de simplecticidad, no podemos incorporar términos de la imagen de $\mathcal{R}$, ya que si lo hacemos, la transformación dejará de representar la transformación a formas normales. En cambio, los términos pertenecientes a $\mathcal{A}^{(\mathrm{N})}$ sí pueden ser incorporados sin restricciones, ya que éstos, al aplicarles el operador $\Delta_{\omega}$, resultan nulos, y no alterarán la forma de $\Phi_{i}^{(\mathrm{N})}$.

Veamos a cuáles de estos conjuntos pertenecen los términos excedentes. Recordemos que el mapa original $\mathcal{F}$ es simpléctico y que, por definición, el nuevo mapa $U$ también lo es. Esto hace que las transformaciones $\Phi_{i}$ sean canónicas. La ecuación homológica, obtenida en la Sección 4.4.4 (Ec. (4.49)), proporciona como solución un conjunto de términos de $\Phi_{i}$ que no pertenecen al núcleo. Cabe destacar que esta solución proporcionada por la ecuación homológica es única. Esto quiere decir que, cualquier término que rompa la condición de simplecticidad deberá pertenecer al núcleo, ya que si no este término pertenecería a la imagen, incumpliendo la unicidad de la solución de la Ec. (4.49).

En base a lo mencionado anteriormente, si bien la ecuación homológica proporciona un conjunto solución único de términos que no pertenecen al núcleo, permite una total libertad en la elección de términos pertenecientes al núcleo para $\Phi_{i}$ dado que, cuando el operador $\Delta_{\omega}$ actúa sobre estos términos, el resultado obtenido es cero.

Antes de incorporar estos términos deberemos tener en cuenta lo siguiente. Los términos que conforman las expresiones de las transformaciones $\Phi_{i}^{(\mathrm{N})}$ aparecen, en la condición de simplecticidad, derivados con respecto a las distintas variables. Luego, los nuevos términos que debemos incorporar a $\Phi_{i}^{(N)}$ deberán cumplir que, al ser derivados en el corchete de Poisson, sean iguales pero de signos opuestos a los términos excedentes de la Ec. (4.61), los cuales solo aparecen en los corchetes $\left[\Phi_{x_{i}}, \Phi_{y_{i}}\right], i=1,2$.

Recordemos que uno de los términos del corchete es de la forma

$$
\frac{\partial \Phi_{x_{i}}^{(N)}}{\partial x_{i}} \frac{\partial \Phi_{y_{i}}^{(N)}}{\partial y_{i}}
$$


con $i=1,2$. Dado que el primer término de $\Phi_{\chi_{i}}^{(N)}$ es $x_{i}$, uno de los términos del corchete será directamente la derivada de $\Phi_{y_{i}}^{(\mathrm{N})}$ con respecto a $y_{i}$ (Ec. (4.62)). Luego, para obtener el término que compensa al excedente, podemos integrar a éste con respecto a $y_{i}$ y sumar su opuesto a $\Phi_{y_{i}}^{(N)}$ de manera que, al calcular el corchete, este término incorporado se cancele con el excedente.

Luego de evaluar y compensar los términos que hacen que la transformación no sea simpléctica, tendremos la transformacion $\Phi_{i}$ y la forma normal $u_{i}$ calculada hasta orden $\mathcal{O}(\mathrm{N})$, donde $\Phi_{i}$ respeta la condición de simplecticidad hasta orden $\mathcal{O}(\mathrm{N})$.

\subsubsection{Transformación inversa}

Como hemos visto en la Ec. (4.38) las transformaciones $\Phi_{i}$ halladas nos permiten expresar las variables originales en términos de las variables nuevas.

Cuando queremos estudiar la dinámica de un sistema calculamos, a partir de las ecuaciones del mapa (o del flujo hamiltoniano, en el caso de sistemas continuos), la serie de tiempo de la evolución de las condiciones iniciales en el conjunto de variables $\left(\mathrm{J}_{1}, \mathrm{~J}_{2}, \mathrm{x}_{1}, \mathrm{x}_{2}\right)$. Pero, cuando el parámetro perturbativo es suficientemente pequeño, estas variables no logran describir la dinámica del sistema, debido a las oscilaciones producidas por los efectos de deformación. Es por este motivo que recurrimos a las formas normales. En este nuevo mapa tenemos un conjunto distinto de variables $\left(\Lambda_{1}, \Lambda_{2}, \theta_{1}, \theta_{2}\right)$ que permitirán continuar con el estudio que, en las variables originales, no se logró realizar. Para obtener el valor numérico de la serie de tiempo en las variables nuevas, es necesario construir la transformación inversa a $\boldsymbol{\Phi}$ para poder expresar las nuevas variables en función de las originales. Esta transformación, que llamaremos $\Psi$, expresa las acciones y ángulos nuevos en términos de los originales,

$$
\left\{\begin{array}{l}
\Lambda_{1}=\Psi_{1}\left(J_{1}, J_{2}, x_{1}, x_{2}\right) \\
\Lambda_{2}=\Psi_{2}\left(J_{1}, J_{2}, x_{1}, x_{2}\right) \\
\theta_{1}=\Psi_{3}\left(J_{1}, J_{2}, x_{1}, x_{2}\right) \\
\theta_{2}=\Psi_{4}\left(J_{1}, J_{2}, x_{1}, x_{2}\right) .
\end{array}\right.
$$

Este nuevo conjunto de transformaciones $\Psi_{i}$ cumplirá que

$$
\left(\mathrm{J}_{1}, \mathrm{~J}_{2}, \mathrm{x}_{1}, \mathrm{x}_{2}\right)=\boldsymbol{\Phi}\left(\Lambda_{1}, \Lambda_{2}, \theta_{1}, \theta_{2}\right)=(\boldsymbol{\Phi} \circ \boldsymbol{\Psi})\left(\mathrm{J}_{1}, \mathrm{~J}_{2}, \mathrm{x}_{1}, \mathrm{x}_{2}\right) .
$$

Este resultado implica que las funciones $\Phi_{i}$ y $\Psi_{i}$ deben ser funciones inversas mutuamente. El procedimiento para calcularlas es el siguiente. Dado que la 
función $\Psi_{i}$ es inversa a $\Phi_{i}$, y que ésta es cercana a la identidad, $\Psi_{i}$ también lo será, y por lo tanto podemos reagrupar sus términos en función de su tamaño utilizando $\lambda$,

$$
\Psi_{i}=\Psi_{i}^{(0)}+\lambda \Psi_{i}^{(1)}+\lambda^{2} \Psi_{i}^{(2)}+\ldots, \quad i=1, \ldots, 4,
$$

donde $\Psi_{i}^{(j)}=\Psi_{i}^{(j)}\left(J_{1}, J_{2}, x_{1}, x_{2}\right)$ y $\left(\Psi_{1}^{(0)}, \Psi_{2}^{(0)}, \Psi_{3}^{(0)}, \Psi_{4}^{(0)}\right)=\left(\Lambda_{1}, \Lambda_{2}, \theta_{1}, \theta_{2}\right)$. Tomemos, por ejemplo, la transformación correspondiente a la variable $\mathrm{J}_{i}$. El proceso para las variables $x_{i}$ es análogo. Por definición, $\Phi_{i}$ y $\Psi_{i}$ son funciones inversas una de la otra por lo que podemos escribir a $J_{i}$ como

$$
\mathrm{J}_{\mathrm{i}}=\left(\Phi_{\mathrm{i}} \circ \boldsymbol{\Psi}\right)\left(\mathrm{J}_{1}, \mathrm{~J}_{2}, \mathrm{x}_{1}, \mathrm{x}_{2}\right)=\Phi_{\mathfrak{i}}\left(\Psi_{1}, \Psi_{2}, \Psi_{3}, \Psi_{4}\right)=\Phi_{\mathfrak{i}}\left(\boldsymbol{\Psi}\left(\mathrm{J}_{1}, \mathrm{~J}_{2}, \mathrm{x}_{1}, \mathrm{x}_{2}\right)\right) .
$$

Si desarrollamos $\Phi_{i}$ y $\Psi_{i}$ como series de potencias de $\lambda$ alrededor del origen y agrupamos sus términos en potencias de $\lambda$ obtenemos

$$
\begin{aligned}
J_{i} & =\left(\sum_{j=0}^{\infty} \lambda^{j} \Phi_{i}^{(j)}\right) \circ\left(\sum_{k=0}^{\infty} \lambda^{k} \Psi^{(k)}\right)= \\
& =\left(\Phi_{i}^{(0)}+\lambda \Phi_{i}^{(1)}+\ldots\right) \circ\left(\Psi^{(0)}+\lambda \Psi^{(1)}+\ldots\right) .
\end{aligned}
$$

Se puede ver claramente que de la composición de un término $\lambda^{j} \Phi_{i}^{(j)}$ con un término $\lambda^{k} \boldsymbol{\Psi}^{(k)}$ se obtiene un término de orden $\mathcal{O}(\boldsymbol{j}+\mathrm{k})$ en $\lambda$.

La resolución de la Ec. (4.67) la llevamos a cabo mediante un procedimiento iterativo donde un dado orden $\mathcal{O}(\mathrm{N})$ se resuelve utilizando los resultados de órdenes $\mathcal{O}(\leqslant N-1)$. La resolución para el orden $\mathcal{O}(0)$ es trivial. Dado que los términos involucrados en este paso deberán cumplir que $j+k=0$, esto sólo ocurrirá cuando $j=k=0$. En este caso, el único término en el miembro derecho que cumple con dicha condición es $\Phi_{i}^{(0)}\left(\boldsymbol{\Psi}^{(0)}\right)=J_{i}$, que coincide con el único término del miembro izquierdo.

Continuemos ahora con la construcción para el orden $\mathcal{O}(1)$. Los términos que contribuyen en este paso son aquéllos en los que $j+k=1$, esto es $j=0 \mathrm{y}$ $\mathrm{k}=1$, o bien $\mathrm{j}=1 \mathrm{y} \mathrm{k}=0$. Sumando todos los términos que cumplen con esta condición e igualándolos a cero (dado que no hay términos de orden $\mathcal{O}(>0)$ en el miembro izquierdo), la ecuación resultante es

$$
\Phi_{i}^{(0)}\left(\Psi^{(1)}\right)+\Phi_{i}^{(1)}\left(\Psi^{(0)}\right)=0
$$


Componiendo el primer término obtenemos $\Phi_{i}^{(0)}\left(\Psi^{(1)}\right)=\Psi_{i}^{(1)}$, mientras que si hacemos lo mismo con el segundo obtenemos $\Phi_{i}^{(1)}\left(\boldsymbol{\Psi}^{(0)}\right)=\Phi_{i}^{(1)}$. Luego, simplemente debemos resolver la ecuación

$$
\Psi_{i}^{(1)}+\Phi_{i}^{(1)}=0
$$

para $\Psi_{i}^{(1)}$, obteniendo como término de orden $\mathcal{O}(1)$ en la transformación inversa a

$$
\Psi_{i}^{(1)}=-\Phi_{i}^{(1)} .
$$

Continuando con la resolución del algoritmo es posible encontrar una relación recursiva en la solución. Para hallar la función inversa $\Psi$ hasta un orden $\mathcal{O}(\mathrm{M})$ el esquema es

$$
\begin{aligned}
\Psi_{i}^{(0)} & =J_{i} \\
\Psi_{i}^{(M)} & =-\Phi_{i}^{(M)}-\left[\sum_{j=1}^{M-1}\left(\Phi_{i}^{(j)} \circ \Psi^{(k<M)}\right)\left(J_{1}, J_{2}, x_{1}, x_{2}\right)\right]_{M},
\end{aligned}
$$

donde, nuevamente, el operador [ $]_{M}$ es el mismo que el utilizado en la Ec. (4.47), y $\Psi^{(k<M)}$ son todos los términos de la funciones inversas $\Psi_{i}^{(k)}$ de órdenes menores que $M$, los cuales ya han sido calculados en iteraciones anteriores (particularmente en la k-ésima iteración).

Nótese que aquí se ha utilizado el índice $M$ para denotar el orden en el desarrollo en vez de N. Esto es debido a que, en general, el orden $M$ hasta el cual se calcula la transformación inversa $\boldsymbol{\Psi}$ y el orden $\mathrm{N}$ hasta el cual calculamos la transformación directa $\Phi$, podrían no coincidir.

\section{$4 \cdot 5$ Discusión}

En este capítulo hemos introducido el mapa que utilizaremos en esta segunda parte del trabajo de tesis, el CRSSM. También hemos mostrado cómo puede cuantificarse la difusión en este sistema, para distintos valores del parámetro perturbativo. Como consecuencia de estos resultados pudimos ver que, para cierto tamaño del valor perturbativo, la detección de variación en las acciones no es trivial. Para ello hemos introducido una técnica, utilizada ya por otros autores, que nos permite reducir las oscilaciones de la d.c.m. Con esta técnica logramos evidenciar, para valores relativamente pequeños del parámetro perturbativo $\gamma$, un comportamiento secular de la d.c.m. 
Encontramos también que, si el parámetro es demasiado pequeño, esta técnica comienza a ser menos efectiva, debido a que la difusión es cada vez más lenta, y el crecimiento de la d.c.m. queda oculto tras las oscilaciones. Luego, necesitamos de la implementación de otra herramienta que nos permita analizar la difusión para valores del parámetro $\gamma$ muy pequeños.

Se implementó una nueva técnica, llamada transformación a formas normales. Esta técnica solamente existía para el caso de flujos hamiltonianos. Por lo tanto, hemos diseñado un nuevo método para su construcción en el caso de un mapa 4D genérico. Entre sus características más destacables podemos mencionar las siguientes:

- El algoritmo diseñado puede ser aplicado a cualquier mapa 4D genérico del tipo cuasi-torsional.

- Implementa un algoritmo que ordena los términos correspondientes al desarrollo en series de Fourier de las ecuaciones del mapa, mediante la inclusión de un parámetro llamado parámetro de contabilidad.

- La transformación puede ser construida de forma iterativa, gracias al parámetro de contabilidad.

- Dada la forma en que está diseñado el algoritmo, es posible generalizarlo para ser aplicado a sistemas con mayor dimensionalidad. 



\section{APLICACIÓN A UN MAPA SIMPLÉCTICO ${ }_{4} \mathrm{D}$}

\subsection{APLICACIÓN DEL CÁlCULO DE FORMAS NORMALES AL CRSSM}

En el Capítulo 4 se ha visto que en el CRSSM, para valores grandes de la perturbación, la evolución de la d.c.m. en el conjunto original de variables podría presentar un crecimiento sistemático en el cual es posible evidenciar fácilmente una difusión aproximadamente normal. Por ende, esto nos permitiría estimar un coeficiente de difusión (ver panel (a) de la Fig. 4.1). En cambio, cuando el parámetro es muy pequeño (panel (b) de la Fig. 4.I), este comportamiento queda oculto frente a las oscilaciones producidas por los efectos de deformación. El cálculo de la forma normal del mapa servirá para revelar estos comportamientos mediante una transformación adecuada. En esta sección se explicará cómo implementar cada uno de los pasos detallados en la Sección 4.4 para el cálculo de la forma normal en el caso específico del CRSSM.

Comparando las Ecs. (4.14) con las Ecs. (4.24), podemos reescribir las primeras en la forma de las últimas simplemente haciendo el reemplazo

$$
\begin{aligned}
& \tilde{F}_{1}\left(y_{1}, y_{2}, x_{1}, x_{2}\right)=F_{1}\left(x_{1}, x_{2}\right)=\varepsilon f_{1}\left(x_{1}\right)+\gamma f_{3}\left(x_{1}+x_{2}\right)+\gamma f_{3}\left(x_{1}-x_{2}\right) \\
& \tilde{F}_{2}\left(y_{1}, y_{2}, x_{1}, x_{2}\right)=F_{2}\left(x_{1}, x_{2}\right)=\varepsilon f_{2}\left(x_{2}\right)+\gamma f_{3}\left(x_{1}+x_{2}\right)-\gamma f_{3}\left(x_{1}-x_{2}\right)(5 \cdot 1)
\end{aligned}
$$

Si en las ecuaciones para los ángulos (Ec. (4.16)) se reemplazan las acciones $y_{i}^{\prime}$ por las expresiones recién definidas, y recordando que las funciones $\Omega_{\mathfrak{i}}\left(\mathrm{y}_{1}, \mathrm{y}_{2}\right)$ solamente dependen de las acciones, podemos asignar $\Omega_{\mathfrak{i}}\left(y_{1}, y_{2}\right)=y_{i}$ con $i=1,2$, obteniendo

$$
\begin{aligned}
& x_{1}^{\prime}=x_{1}+\Omega_{1}\left(y_{1}, y_{2}\right)+\varepsilon F_{1}\left(x_{1}, x_{2}\right) \\
& x_{2}^{\prime}=x_{2}+\Omega_{2}\left(y_{1}, y_{2}\right)+\varepsilon F_{2}\left(x_{1}, x_{2}\right)
\end{aligned}
$$

a partir de las cuales, comparándolas con las correspondientes para los ángulos de $(4.24)$ se ve que $\tilde{F}_{3}\left(y_{1}, y_{2}, x_{1}, x_{2}\right)=F_{1}\left(x_{1}, x_{2}\right)$ y $\tilde{F}_{4}\left(y_{1}, y_{2}, x_{1}, x_{2}\right)=F_{2}\left(x_{1}, x_{2}\right)$. 
Como podemos ver, las funciones $F_{i}$ que en principio dependen de las dos acciones y los dos ángulos, en nuestro caso sólo dependen de los últimos. Este hecho es muy conveniente para nuestro procedimiento ya que las acciones solo aparecen como términos lineales aditivos. Por lo tanto, no es necesario realizar el desarrollo en series de Taylor de $F_{1}\left(x_{1}, x_{2}\right)$ ni de $F_{2}\left(x_{1}, x_{2}\right)$. De la misma manera, como las funciones $\Omega_{\mathfrak{i}}\left(y_{1}, y_{2}\right)$ tienen una dependencia lineal con las acciones como vimos más arriba, tampoco es necesario realizar su desarrollo. Por último, dado que todos los parámetros perturbativos $\varepsilon_{i}$ del mapa CRSSM son iguales, $\varepsilon_{1}=\varepsilon_{2}=\varepsilon_{3}=\varepsilon_{4}=\varepsilon$.

Una vez que se tienen asociados los términos entre la Ec. (4.17) y la Ec. (4.24) procedemos al siguiente paso, que consiste en realizar el cambio de variables (4.28) para reescribir nuestro mapa en torno al punto central $\left(y_{1}^{*}, y_{2}^{*}\right)$

$$
\begin{aligned}
& J_{1}^{\prime}=J_{1}+\varepsilon\left(\varepsilon f_{1}\left(x_{1}\right)+\gamma\left[f_{3}\left(x_{1}+x_{2}\right)+f_{3}\left(x_{1}-x_{2}\right)\right]\right), \\
& J_{2}^{\prime}=J_{2}+\varepsilon\left(\varepsilon f_{2}\left(x_{2}\right)+\gamma\left[f_{3}\left(x_{1}+x_{2}\right)-f_{3}\left(x_{1}-x_{2}\right)\right]\right), \\
& x_{1}^{\prime}=x_{1}+\omega_{1}^{*}+J_{1}^{\prime}, \\
& x_{2}^{\prime}=x_{2}+\omega_{2}^{*}+J_{2}^{\prime},
\end{aligned}
$$

donde, como hemos mencionado en el capítulo anterior, $\omega_{1}^{*}$ y $\omega_{2}^{*}$ son dos constantes que corresponden al valor del orden cero del desarrollo de Taylor de $\Omega_{\mathfrak{i}}\left(y_{1}, y_{2}\right)$, con $i=1,2$, es decir $\Omega_{\mathfrak{i}}\left(y_{1}^{*}, y_{2}^{*}\right)$. Dado que estas funciones son iguales a su respectiva acción, el orden cero del desarrollo de Taylor de éstas coincide con la acción evaluada en el punto. Para simplificar la notación, escribiremos $\omega_{i}^{*} \rightarrow \omega_{i}$, y sus valores serán simplemente $\omega_{i}=\Omega_{i}\left(y_{1}^{*}, y_{2}^{*}\right)=y_{i}^{*}$.

El próximo paso consiste en implementar el parámetro de contabilidad $\lambda$ en el mapa (5.3). Para ello es necesario primero encontrar la función generatriz $S$ del mapa, cuyo cálculo es inmediato partiendo de la expresión final del mapa expresado en las Ecs. (5.3), y recordando la expresión de las funciones $f_{i}\left(x_{1}, x_{2}\right)$ (Ec. (4.12)). De aquí se obtiene

$$
\begin{aligned}
\mathrm{S}\left(\mathrm{J}_{1}^{\prime}, \mathrm{J}_{2}^{\prime}, \mathrm{x}_{1}, \mathrm{x}_{2}\right)= & \left(\mathrm{x}_{1}+\omega_{1}\right) \mathrm{J}_{1}^{\prime}+\left(\mathrm{x}_{2}+\omega_{2}\right) \mathrm{J}_{2}^{\prime}+\frac{\left(\mathrm{J}_{1}^{\prime 2}+\mathrm{J}_{2}^{\prime 2}\right)}{2}- \\
& -\varepsilon\left[\mathrm{g}\left(\mathrm{x}_{1}, \mathrm{x}_{2}\right)+\mathrm{h}\left(\mathrm{x}_{1}, \mathrm{x}_{2}\right)\right], \\
\mathrm{g}\left(\mathrm{x}_{1}, \mathrm{x}_{2}\right)= & \varepsilon\left(\frac{\ln \left(1-\mu_{1} \cos \left(x_{1}\right)\right)}{\mu_{1}}+\frac{\ln \left(1-\mu_{2} \cos \left(\mathrm{x}_{2}\right)\right)}{\mu_{2}}\right), \\
\mathrm{h}\left(\mathrm{x}_{1}, \mathrm{x}_{2}\right)= & \gamma\left(\frac{\ln \left(1-\mu_{3} \cos \left(x_{1}+x_{2}\right)\right)}{\mu_{3}}+\frac{\ln \left(1-\mu_{3} \cos \left(x_{1}-x_{2}\right)\right)}{\mu_{3}}\right) .
\end{aligned}
$$

Una vez obtenida la función generatriz, efectuamos su desarrollo en serie de Fourier. Podemos ver que la dependencia de la función generatriz con las acciones se produce mediante una expresión polinómica de grado 2, que son los tres primeros términos de la Ec. (5.4). Esto hace que no tengamos que aplicar un 
desarrollo en serie de Taylor para quitar cualquier dependencia con las acciones de las exponenciales del desarrollo de Fourier. Luego, $\mathrm{S}$ toma la forma

$$
\begin{array}{r}
S\left(J_{1}^{\prime}, J_{2}^{\prime}, x_{1}, x_{2}\right)=\left(x_{1}+\omega_{1}\right) J_{1}^{\prime}+\left(x_{2}+\omega_{2}\right) J_{2}^{\prime}+\frac{\left(J_{1}^{\prime 2}+J_{2}^{\prime 2}\right)}{2}+ \\
\sum_{k_{1}, k_{2}=0}^{\infty} \mathcal{C}_{k_{1}, k_{2}} e^{i\left(k_{1} x_{1}+k_{2} x_{2}\right)},
\end{array}
$$

donde los coeficientes $\mathcal{C}_{\mathrm{k}_{1}, \mathrm{k}_{2}}$ dependen de los parámetros $\varepsilon$ y $\gamma$.

Con respecto al parámetro de contabilidad $\lambda$, debemos analizar el orden de cada uno de los términos. Como se ha supuesto en la Sección 4.4.2, el tamaño de $\mathrm{J}_{i}$ es $\sim \sqrt{\varepsilon}$. Luego, podemos suponer que las variables $J_{i}$ son del orden de $\lambda$, realizando entonces la sustitución $J_{i} \rightarrow \lambda J_{i}$ en la Ec. (5.5). Con este cálculo ya queda implementado $\lambda$ en los términos que dependen de $J_{i}$. Con respecto a la dependencia con $\lambda$ de las variables angulares, nos enfocamos en los términos de Fourier. Estos términos decrecen con una tasa exponencial, $e^{-\sigma|K|}$, con $K \in \mathbb{Z}$, la cual podemos suponer que es $\sim \sqrt{\varepsilon}$. Conociendo el tamaño aproximado de $\varepsilon$ y el valor del radio de convergencia $\sigma$, es posible hallar el valor para K. En todos nuestros cálculos se considerará a $\varepsilon$ fijo, siendo su valor $\varepsilon=0,1$. Con respecto a $\sigma$, como las funciones $g\left(x_{1}, x_{2}\right)$ y $h\left(x_{1}, x_{2}\right)$ son sumas de logaritmos, debemos encontrar el radio de convergencia de éstos. Consideremos la función $\log (1-\mu \cos (x))$. Para calcular su radio de convergencia centrado en $x_{0}=0$ debemos buscar en qué valor de $x$ se encuentra la singularidad más próxima. Para esto es necesario ver qué sucede en el plano complejo. Consideramos a $x \in \mathbb{C}$, por lo que el coseno también será una función compleja. Luego, la solución de $1-\mu \cos (x)=0$ es inmediata:

$$
|x|=\left|\arccos \left(\frac{1}{\mu}\right)\right|
$$

Como el mapa posee 3 parámetros $\mu_{i}$, debemos hallar el radio de convergencia para estos 3 valores y tomar la intersección de sus soluciones. Haciendo esto se obtiene, para $\sigma$

$$
\begin{aligned}
& \left|\arccos \left(\frac{1}{\mu_{1}}\right)\right|=1,317, \\
& \left|\arccos \left(\frac{1}{\mu_{2}}\right)\right|=1,567, \\
& \left|\arccos \left(\frac{1}{\mu_{3}}\right)\right|=1,099 .
\end{aligned}
$$


La intersección entre los radios de convergencia coincide con el menor de los valores. Luego, resolviendo para K llegamos a

$$
|\mathrm{K}|=-\frac{\ln \sqrt{\varepsilon}}{\sigma} \approx 1,048 .
$$

Como debemos considerar el entero más cercano al valor obtenido, adoptamos el valor $K=1$. Esto significa que el tamaño de los términos de Fourier van linealmente con $\lambda$, es decir que, por cada orden del desarrollo de Fourier, la potencia de $\lambda$ aumenta en 1 .

Para la implementación numérica de las ecuaciones del mapa CRSSM como series de Fourier, hemos considerado un conjunto genérico de reglas, para $F_{i}$, $\Phi_{i}$ y $U_{i}$, que deben obedecer los índices (para conocer las reglas específicas de cada función, consultar el Apéndice B). Estas reglas generales son

- $0 \leqslant s_{1}+s_{2} \leqslant r$

- $-r \leqslant k_{1}, k_{2} \leqslant r$

- $r_{\text {mín }}(r) \leqslant s_{1}+s_{2}+\left|k_{1}\right|+\left|k_{2}\right| \leqslant r_{\text {máx }}(r)$.

A partir de estas relaciones podemos definir los valores de $r_{\text {mín }}(r)$ y $r_{\text {máx }}(r)$, como hemos mencionado en la Sección 4.4.2. Considerando el valor límite inferior que pueden tomar los índices (es decir, $s_{1}+s_{2}=\left|k_{1}\right|=\left|k_{2}\right|=0$ ), obtenemos $r_{\text {mín }}(r)=0$. De la misma manera, tomando los límites superiores $\left(s_{1}+s_{2}=\left|k_{1}\right|=\left|k_{2}\right|=r\right)$, obtenemos $r_{\text {máx }}(r)=3 r$.

Dado que, para caracterizar unívocamente cada término del desarrollo de Fourier, son necesarios 5 índices, su implementación numérica puede llevarse a cabo mediante la utilización de un arreglo de 5 dimensiones, donde el contenido del elemento es el coeficiente del término correspondiente. Esta implementación insume una cantidad excesiva de memoria, dado que muchos de sus elementos (correspondientes a combinaciones de los índices que no aparecen en la serie) pueden ser nulos y por lo tanto no son utilizados en el algoritmo.

En lugar de utilizar un arreglo de 5 dimensiones para almacenar los coeficientes, hemos implementado una función de indexación para este fin. El objetivo de esta función es, a partir de los 5 índices correspondientes a cada término de la serie $\left(s_{1}, s_{2}, k_{1}, k_{2}\right.$ y r), generar un único índice ind que los caracteriza. Esto hace que sólo se utilice un arreglo unidimensional, disminuyendo la cantidad de memoria necesaria (ver Apéndice B para su construcción).

Para implementar la ecuación de conjugación (Ec. (4.43)) en este mapa en particular, las únicas funciones conocidas son $F_{i}$. Tanto $U_{i}$ como $\Phi_{i}$ son desconocidas, por lo que tomarán la forma general de las Ecs. (4.4.3) Y (4.40), respectivamente. Llevando a cabo las composiciones de la Ec. (4.43) (ver Apéndice A obtendremos la ecuación homológica (4·49). 
Una vez que tenemos la ecuación homológica debemos construir las funciones $\boldsymbol{\Phi}$ y $\boldsymbol{U}$ a partir de la imagen $\mathcal{R}$ y el núcleo $\mathcal{N}$ del operador de diferencias (4.50), como se muestra en la Ec. (4.58).

Para construir las funciones debemos analizar, uno a uno, cada término de $\mathrm{Q}_{i}^{(\mathrm{N})}$ y se los clasifica, analizando cuáles de ellos pertenecen al núcleo del operador lineal de diferencias y cuáles pertenecen a su imagen.

Planteando la Ec. (4.53) para un término de $U_{i}$ obtuvimos que

$$
e^{i\left(k_{1} \omega_{1}+k_{2} \omega_{2}\right)}-1=0,
$$

cuya solución es $k_{2}=-\left(\omega_{1} / \omega_{2}\right) k_{1}$.

Como el método para el cálculo de la forma normal del mapa desarrollado en el capítulo anterior es el correspondiente a una resonancia simple, es necesario encontrar una única terna de valores $\left(k_{1}, k_{2}, k_{3}\right)$ que satisfagan la condición de resonancia (Ec. (4.19)). De la aplicación de la solución recién hallada en la Ec. (4.19) tenemos que $k_{3}=0$, y dado que la resonancia que estamos considerando es la $1: 1$, obtenemos que $\omega_{1}=\omega_{2}=\omega$, implicando $k_{2}=-k_{1}$. Para este trabajo hemos adoptado para la frecuencia el valor $\omega=\sqrt{3}$, el cual puede verse que satisface las condiciones requeridas.

Para realizar la comprobación de simplecticidad, simplemente se sigue la explicación de la Sección 4.4.5, calculando los corchetes de Poisson. Si en la tranformación aparece algún término no nulo de orden $\mathcal{O}(\leqslant N)$, debemos compensar su aparición como se detalla en la Sección 4·4·5.

Finalmente, debemos calcular la transformación inversa. El procedimiento es el mismo que el visto en la Sección 4.4.6, teniendo que calcular básicamente la Ec. (4.71). La implementación de este algoritmo es bastante directa. Para construir la función $\Psi_{i}$ de orden $\mathcal{O}(M)$ tenemos, por un lado, un término que consiste en la función $\Phi_{i}$ (también a orden $\mathcal{O}(M)$ ) con signo opuesto, y por otro lado tenemos la sustracción de una sumatoria, cuyos elementos son el resultado de la composición de $\Phi_{i}$ con las funciones $\Psi_{j}$, donde $\Psi_{j}$ son las transformaciones inversas calculadas hasta el orden $\mathcal{O}(M-1)$.

Para la implementación numérica de este método, se construyó un código, escrito en Fortran 90, que calcula la transformación a formas normales para el CRSSM. En el Apéndice C se muestran los detalles de su implementación.

\subsection{RESULTADOS}

Una vez implementado el método de las formas normales al CRSSM, es importante conocer sus capacidades y limitaciones. Esta sección será dedicada a 
presentar resultados obtenidos luego de aplicar la implementación vista en la Sección 5.1.

Uno de los objetivos de esta sección es estimar el tiempo necesario para el cálculo de un orden específico de la transformación a formas normales. Para ello, lo que se hace es calcular dicha transformación para distintos órdenes del desarrollo, y luego comparar la variación de los tiempos de CPU de las distintas ejecuciones del código presentado en el final de la Sección 5.1. Con estos resultados intentaremos buscar una dependencia del tiempo de CPU con el orden del desarrollo.

El otro objetivo de esta sección es calcular, a partir de las transformaciones para los distintos órdenes, si existe una dependencia entre la disminución de las oscilaciones en las acciones con el orden $\mathrm{N}$ de la transformación, intentando posteriormente hallar, de manera empírica, el llamado orden óptimo $\mathrm{r}_{\mathrm{opt}}$ (ver, por ejemplo, [18], [28], [25], [26], [30], [29], entre otros) el cual será definido más adelante.

Todos los cálculos realizados en este capítulo, como también en los capítulos siguientes, fueron realizados con la misma configuración de hardware que la descripta en la Sección 3.7.1.

\subsubsection{Tiempos de cómputo}

Como se muestra en el Apéndice $C$, el paquete implementado para el cálculo de la forma normal consta de tres programas que funcionan de manera encadenada: el primero construye el desarrollo en serie de Fourier del mapa $\mathcal{F}$; el segundo programa es el encargado de construir, a partir de la salida del primer programa, la transformación de la forma normal $\Phi$, el nuevo mapa $\mathcal{U}$ y la transformación inversa $\boldsymbol{\Psi}$; por último, el tercer programa, a partir de la transformación inversa $\boldsymbol{P} \boldsymbol{s i}$ calculado con el programa anterior y con la evolución de la órbita en el espacio de fases del mapa original, construye la serie de tiempo transformada, en las variables $\left(\Lambda_{1}, \Lambda_{2}, \theta_{1}, \theta_{2}\right)$, junto con el valor de la d.c.m. para $I_{f}$ e $I_{R}$, en ambos conjuntos de variables.

En esta sección se mostrará el tiempo de CPU insumido por el segundo y el tercer programa de nuestro paquete. El tiempo de CPU empleado por el primer programa para construir la serie de Fourier de $\boldsymbol{F}\left(\mathrm{J}_{1}, \mathrm{~J}_{2}, \mathrm{x}_{1}, \mathrm{x}_{2}\right)$ no es analizado, ya que los valores de aquél son demasiado pequeños como para ser tenidos en cuenta ( 1s para la construcción del desarrollo hasta orden $\mathcal{O}(15))$.

Los tiempos de CPU para los programas antes mencionados, fueron calculados con la misma configuración de hardware que la empleada en la Sección 3.7.1.

Para la configuración que define nuestro mapa hemos considerado: 
- $\varepsilon=0,1$.

- $\gamma=0,05$.

- $\omega_{1}^{*}=\omega_{2}^{*}=\sqrt{3}$ (lo que hace que $\left.y_{1}^{*}=y_{2}^{*}=\sqrt{3}\right)$.

- Un conjunto de $\mathrm{N}_{\mathrm{ci}}=10^{2}$ condiciones iniciales alrededor del punto $\left(y_{10}, y_{20}, x_{10}, x_{20}\right)=(1,75,1,75,0,0)$, escogidas al azar en un disco de radio $10^{-3}$.

- Orden de desarrollo $N$ en el rango $1 \leqslant N \leqslant 12$.

- Número de iteraciones: $\mathrm{N}_{\text {iter }}=10^{7}$.

- Paso de escritura: $10^{4}$ (para un total de $10^{3}$ datos de salida).

En la Tabla 5.I se representan los valores de tiempo de CPU ( $\left.t_{\mathrm{CPU}}\right)$, medidos en segundos, empleados en la ejecución de los programas mencionados más arriba. La primera columna de la tabla muestra cada uno de los órdenes del desarrollo para los que se corrió el programa. La segunda columna muestra el $t_{\mathrm{CPU}}$ insumido para la ejecución del programa que calcula las transformaciones $\boldsymbol{\Phi}$ y $\boldsymbol{\Psi}$ y el mapa $\mathcal{U}$ para cada orden del desarrollo. Por último, la tercera columna muestra el $t_{\mathrm{CPU}}$, por orden de desarrollo, insumido por el programa que construye la nueva serie temporal junto con el cálculo de la d.c.m. $\left(\eta^{2}\right)$.

Los resultados de la Tabla 5.1 muestran que los tiempos para los órdenes 1, 2 y 3 están en el rango $0<t_{\mathrm{CPU}}<5 \mathrm{~s}$ para el cálculo del segundo programa, y $0<\mathrm{t}_{\mathrm{CPU}}<2 \mathrm{~s}$ para el cálculo del tercer programa. Estos valores son muy pequeños, por lo que el error relativo puede ser alto. Es por esto que serán descartados de los estudios que siguen a continuación.

Los tiempos de CPU para la forma normal, entre los órdenes $\mathcal{O}(4)$ y $\mathcal{O}(8)$, presentan un incremento en un factor aproximadamente 1,5 por orden mientras que, a partir del orden 9, los tiempos presentan un aumento considerable, creciendo hasta un factor aproximadamente 5,5 por orden. En la Fig. 5.1 está reflejado este comportamiento, donde se representan los resultados de la segunda columna de la Tabla 5.1.

En esta figura, los puntos representan el logaritmo natural del $t_{\mathrm{CP}}$ para el cálculo de la forma normal a distintos órdenes $\mathrm{N}$, desde $\mathcal{O}(4)$ hasta $\mathcal{O}(12)$. La elección de esta escala fue debido a la gran diferencia en órdenes de magnitud entre los tiempos de cómputo para los distintos órdenes del desarollo. La distribución de estos puntos parece presentar un perfil de crecimiento bilineal en escala logarítimica. En escala lineal este comportamiento bilineal debe entenderse como un comportamiento exponencial con dos exponentes distintos: uno para el rango $4 \leqslant N \leqslant 8$ y otro para el rango $9 \leqslant N \leqslant 12$. Mediante el método 


\begin{tabular}{|c|r|r|}
\hline Orden & $\mathrm{t}_{\mathrm{CPU}}$ & $\mathrm{t}_{\mathrm{CPU}}$ \\
\hline 1 & 0,44 & 0,87 \\
2 & 2,03 & 1,12 \\
3 & 4,82 & 1,68 \\
4 & 8,93 & 2,96 \\
5 & 14,02 & 6,07 \\
6 & 20,62 & 12,14 \\
7 & 29,63 & 23,70 \\
8 & 48,04 & 43,83 \\
9 & 137,70 & 75,35 \\
10 & 759,24 & 124,57 \\
11 & 4393,17 & 194,73 \\
12 & 24723,26 & 295,52 \\
\hline
\end{tabular}

Tabla 5.1: Tiempo de CPU en función del orden del desarrollo para la construcción de la transformación $\boldsymbol{\Phi}$, la transformación inversa $\boldsymbol{\Psi}$ y la forma normal $\mathcal{U}$ (segunda columna), y para el cálculo de la desviación cuadrática media (d.c.m.) (tercera columna), para los órdenes $1 \leqslant \mathcal{O}(N) \leqslant 12$, medido en segundos.

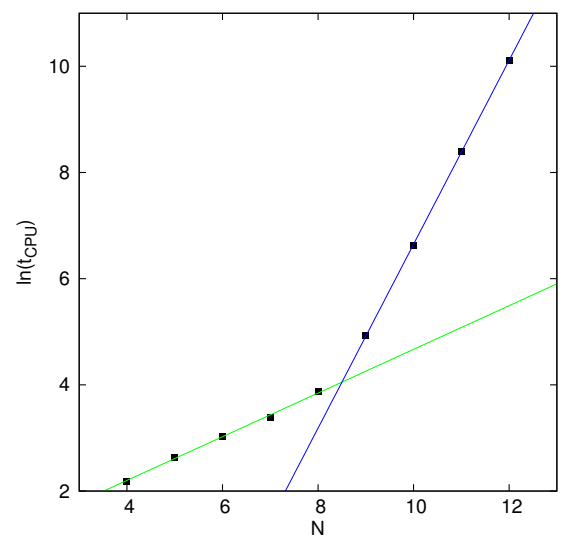

Figura 5.1: $\ln \left(t_{\mathrm{CPU}}\right)$ (medido en segundos) vs. orden $(\mathrm{N})$ de la transformación para el cálculo de la transformación $\Phi$, la transformación inversa $\boldsymbol{\Psi}$ y la forma normal $U$. La recta verde corresponde al ajuste $\ln \left(\mathrm{t}_{\mathrm{CPU}}(\mathrm{N})\right)=0,411 \mathrm{~N}+$ 0,555 en el rango $4 \leqslant N \leqslant 8$, mientras que la recta azul corresponde al ajuste $t_{C P U}(N)=1,733 N-10,678$ en el rango $9 \leqslant N \leqslant 12$.

de mínimos cuadrados podemos realizar el ajuste de dos funciones lineales de la forma $\ln \left(t_{\mathrm{CPU}}\right)=\mathrm{mN}+\mathrm{a}$, una para cada comportamiento, donde $\mathrm{m}$ y a son 
constantes a determinar, y de aquí estimar su pendiente. Este valor nos dará información de la tasa de crecimiento $(\mathrm{m})$ del tiempo de CPU. El ajuste para $4 \leqslant N \leqslant 8$ nos da una estimación de la pendiente de $m=0,411$. Con el valor estimado para $m$ llegamos a que la dependencia del $t_{C P U}$ con $N$ es aproximadamente $t_{\mathrm{CPU}} \propto 1,509^{\mathrm{N}}$. Si hacemos un razonamiento similar para los tiempos comprendidos entre los órdenes $\mathcal{O}(9)$ y $\mathcal{O}(12)$, obtenemos una estimación de la pendiente $\mathrm{m}=1,733$, proporcionando una dependencia entre $t_{\mathrm{CPU}} \mathrm{y} \mathrm{N}$ del tipo $t_{\mathrm{CPU}} \propto 5,656^{\mathrm{N}}$.

En cuanto a la medición de los $t_{\mathrm{CPU}}$ en el cálculo de la d.c.m., la Fig. 5.2 muestra los valores de la tercera columna de la Tabla 5.I en función del orden $\mathrm{N}$, los cuales parecen seguir un perfil tipo exponencial. De la misma manera que se hizo para los tiempos de CPU de la forma normal, mediante mínimos cuadrados se realizó un ajuste de la forma $t_{C P U}(N)=a e^{b N}+c$. El valor de c es simplemente un corrimiento de la curva, y a es un factor constante que amplifica (si $a>1)$ o reduce (si $a<1)$ la velocidad con la que crece la curva. En cambio, b es la cantidad más relevante de este ajuste, ya que su valor indica la velocidad con la cual crecen los tiempos en función del orden. Mediante sus estimaciones obtenemos que el factor de amplificación del crecimiento es $a=2,073$ y la velocidad de crecimiento es $b=0,417$, lo que resulta en una dependencia del $t_{C P U}$ con $\mathrm{N}$ dada por $t_{C P U} \propto 2,073 \times 1,517^{\mathrm{N}}$.

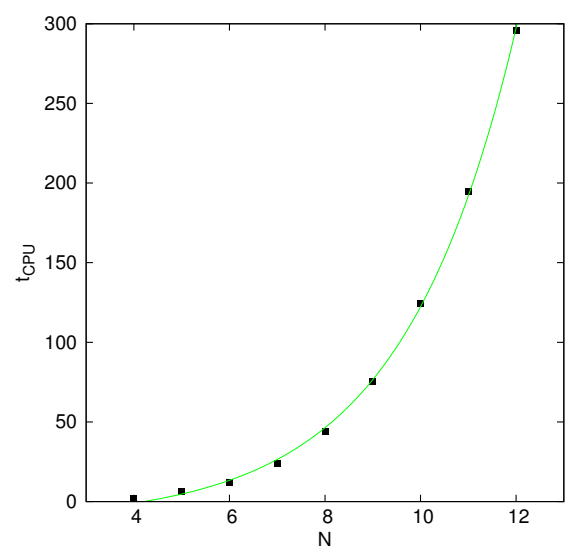

Figura 5.2: $t_{\mathrm{CPU}}$ (medido en segundos) vs. orden $\mathcal{O}(\mathrm{N})$ de la transformación $\Phi$ para el cálculo de la d.c.m. La curva representa el ajuste de una función exponencial, $t_{\mathrm{CPU}}(\mathrm{N})=2,073 \mathrm{e}^{0,417 \mathrm{~N}}-11,967$.

A partir de estos dos gráficos es posible tener una noción de cómo será la progresión de los tiempos de cálculo para ambos programas. En el caso particular del cálculo de la forma normal, es difícil predecir el tiempo que insumirá el programa para un orden $\mathrm{N}>12$, dado que en la dependencia temporal 
con $\mathrm{N}$ se produce un cambio de comportamiento entre $\mathrm{N}=8$ y $\mathrm{N}=9$. Esto hace que no podamos asegurar un comportamiento determinado fuera de los órdenes calculados, siendo por ende difícil extrapolar el tiempo que insumirá una ejecución a órdenes $>12$. Con respecto al tiempo insumido en el cálculo de la d.c.m., si suponemos correcta la estimación de la dependencia de $t_{\mathrm{CPU}}$ con $\mathrm{N}$ como una función exponencial, podemos predecir, con un error tal vez pequeño, el tiempo que empleará una ejecución del programa para un orden arbitrario $\mathrm{N}$.

Desde un punto de vista cuantitativo, estos resultados sugieren que, para el cálculo de la d.c.m., el tiempo de CPU es una cantidad pequeña en comparación con los tiempos empleados para el cálculo de la forma normal, permitiéndonos realizar el cálculo hasta un orden $\mathcal{O}(12)$ en un tiempo del orden de 5 minutos.

Como hemos visto en la Fig. 5.1, a partir del orden $N=9$, al aumentar un orden el desarrollo de $\Phi$, los tiempos de cómputo se incrementan de manera más acelerada que para $N \leqslant 8$. Por ende, sería deseable que sea suficiente con realizar el desarrollo hasta un orden $\mathrm{N}<9$. Una posible explicación a este cambio brusco en la velocidad de crecimiento de los tiempos de cómputo puede ser el siguiente. Dado que el aumento del orden del desarollo provoca un incremento en el número de términos del desarrollo de Fourier y, por consiguiente, en el número de operaciones, a partir del orden $\mathrm{N}=9$ es posible que el programa necesite reservar más memoria que la disponible en la computadora, haciendo que el sistema operativo deba utilizar parte del espacio en disco (que es considerablemente más lento que la memoria RAM) como memoria de intercambio, provocando un incremento en el $t_{\mathrm{CPU}}$. Como este hecho no interfiere con los resultados de nuestro trabajo, no hemos profundizado la búsqueda de su origen, quedando como un posible trabajo a futuro.

En la siguiente sección vamos a ver que existe un orden para el cual el desarrollo es óptimo, dado que su error alcanza un mínimo y, si quisiéramos aumentar el orden del desarrollo más allá de este orden óptimo, los errores se incrementarían progresivamente.

\subsubsection{Precisión de la transformación y estimación empírica del orden óptimo}

En la sección anterior se han presentado los tiempos de CPU que emplean en ejecutarse los programas implementados. Se ha estimado también una relación funcional de estos tiempos con respecto al orden $\mathrm{N}$ del desarrollo. Sin embargo, no siempre es conveniente realizar la transformación con un orden arbitrariamente alto, sino que existe un valor, llamado orden óptimo, el cual nos indica para qué valor de $\mathrm{N}$ la transformación se calcula con mayor precisión. 
En el nuevo conjunto de variables, el mapa se puede expresar de la forma

$$
\begin{aligned}
& \Lambda_{1}^{\prime}=u_{\Lambda_{1} \leqslant r}\left(\Lambda_{1}, \Lambda_{2}, \theta_{1}, \theta_{2}\right)+R_{\Lambda_{1}}^{(r)}\left(\Lambda_{1}, \Lambda_{2}, \theta_{1}, \theta_{2}\right) \\
& \Lambda_{2}^{\prime}=u_{\Lambda_{2} \leqslant r}\left(\Lambda_{1}, \Lambda_{2}, \theta_{1}, \theta_{2}\right)+R_{\Lambda_{2}}^{(r)}\left(\Lambda_{1}, \Lambda_{2}, \theta_{1}, \theta_{2}\right) \\
& \theta_{1}^{\prime}=u_{\theta_{1 \leqslant r}}\left(\Lambda_{1}, \Lambda_{2}, \theta_{1}, \theta_{2}\right)+R_{\theta_{1}}^{(r)}\left(\Lambda_{1}, \Lambda_{2}, \theta_{1}, \theta_{2}\right) \\
& \theta_{2}^{\prime}=u_{\theta_{2} \leqslant r}\left(\Lambda_{1}, \Lambda_{2}, \theta_{1}, \theta_{2}\right)+R_{\theta_{2}}^{(r)}\left(\Lambda_{1}, \Lambda_{2}, \theta_{1}, \theta_{2}\right),
\end{aligned}
$$

donde las funciones $U_{\Lambda_{i} \leqslant r}, U_{\theta_{i} \leqslant r}$, con $i=1,2$ son las ecuaciones de la forma normal del nuevo mapa obtenido mediante la transformación $\boldsymbol{\Phi}$ hasta un orden $r$ en el parámetro de contabilidad $\lambda$. Las funciones $R_{\Lambda_{1}}^{(r)}, R_{\Lambda_{2}}^{(r)}, R_{\theta_{1}}^{(r)}$ y $R_{\theta_{2}}^{(r)}$, resumidas en su expresión vectorial $\boldsymbol{R}^{(\mathrm{r})}\left(\Lambda_{1}, \Lambda_{2}, \theta_{1}, \theta_{2}\right)$, son el llamado residuo de la forma normal ([18], [30]). Cada una de las componentes de $\boldsymbol{R}^{(\mathrm{r})}$ está formada por una serie de Fourier de todos los términos de orden mayor o igual que $r+1$ en $\lambda$ producidos por los términos del mapa original $\mathcal{F}$. El tamaño del residuo nos indica el error con el que se está calculando la forma normal, truncada a este orden. Si bien es cierto que, para órdenes bajos, a medida que aumenta el orden $r$ de la transformación el residuo disminuye, existe un orden, llamado orden óptimo $r_{\mathrm{opt}}$ a partir del cual el residuo comienza a incrementarse para valores de $r>r_{\text {opt }}$. La razón por la cual se produce este incremento en el residuo es la siguiente. Recordemos de la Ec. (4.53) que, si un término de $Q_{i}^{(N)}$ no pertenece al núcleo $\mathcal{N}$ de $\Delta_{\omega}$, debemos dividirlo por $e^{i\left(k_{1} \omega_{1}^{*}+k_{2} \omega_{2}^{*}\right)}-1$ para que tenga la forma de un elemento de $\mathcal{R}$, y pueda ser asignado como un término de $\Phi_{i}$. Luego, a medida que consideramos transformaciones de órdenes superiores, estos denominadores serán cada vez más pequeños y, eventualmente, existirá un orden $\left(\mathrm{r}_{\mathrm{opt}}\right)$ a partir del cual, el tamaño en que se reducen las oscilaciones debido al aumento del orden del desarrollo es menor que el error introducido por estos pequeños denominadores.

En [18] y [30], los autores calculan el valor de $r_{\text {opt }}$ a partir del cálculo del residuo. En esta sección no se calculará el residuo, sino que se estimará el valor de $r_{o p t}$ de forma enteramente empírica, a partir de la comparación de las curvas de evolución de la d.c.m. para los distintos órdenes. Esta estimación empírica del orden óptimo se realizará para los puntos centrales $y_{1}^{*}=y_{2}^{*}=$ $\sqrt{3}+\delta$, tomando dos valores distintos de $\delta$.

Recordemos que la transformación a formas normales $\boldsymbol{\Phi}$ presentada en el Capítulo 4 , corresponde a un desarrollo en torno a un punto $\left(y_{1}^{*}, y_{2}^{*}\right)$ perteneciente a una resonancia simple. Como estas transformaciones tienen carácter local, debe existir un pequeño entorno de este punto que también pertenezca a la resonancia simple para que su aplicación tenga validez. 
Hemos escogido dos valores de $\left(y_{1}^{*}, y_{2}^{*}\right)$ sobre la resonancia $1: 1$ en torno a los cuales construir la forma normal. Uno de estos puntos, con $\delta=0,08$, lo hemos elegido en una región donde claramente se encuentra en una resonancia simple, lejos de un cruce de resonancias $^{1}$, como puede verse en las Figs. 5.3. El otro punto se eligió de manera que, si bien estará también en una resonancia simple, se encuentre más cerca de un cruce. Para este punto se eligió $\delta=0$. En el panel (a) de la Fig. 5.3, correspondiente a $\gamma=0,1$ puede notarse que dicho punto pertenece a una resonancia simple, pero se encuentra lo suficientemente cerca de un cruce y, por lo tanto, cerca de una región de superposición de resonancias. En el panel (b) de la Fig. 5.3 se muestra la misma situación que en el panel (a) para $\gamma=0,01$. Debido al tamaño del parámetro $\gamma$ los anchos de las resonancias disminuyen, disminuyendo también la región donde se produce la superposición de resonancias, y el punto correspondiente a $\delta=0$ puede considerarse que se encuentra en una resonancia simple al igual que para $\delta=$ 0,08 .
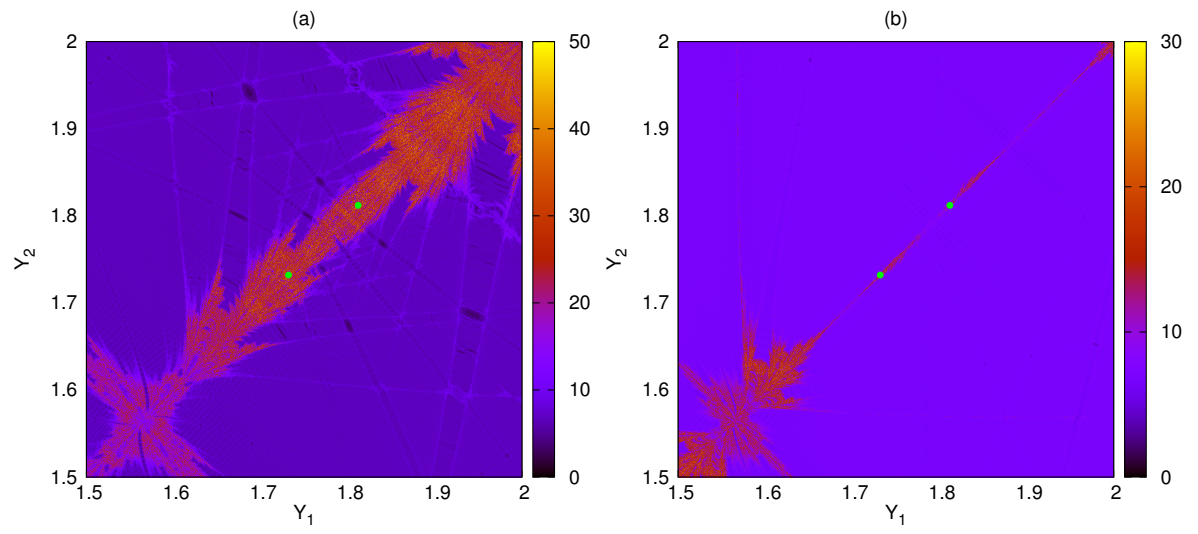

Figura 5.3: Mapa del $\ln (\mathrm{FLI})$ en el plano $\left(\mathrm{y}_{1}, \mathrm{y}_{2}\right)$ para $1000 \times 1000$ condiciones iniciales en la región $[1,5 ; 2,0] \times[1,5 ; 2,0]$, con $\gamma=0,1$ (a) y $\gamma=0,01$ (b). El extremo inferior de la escala de colores (violeta) corresponde a órbitas regulares, mientras que el extremo superior (amarillo) corresponde a órbitas caóticas. Los puntos sobre la resonancia representan las acciones centrales, alrededor de las cuales se realiza el desarrollo: $y_{1}^{*}=y_{2}^{*}=\sqrt{3}+\delta$, con $\delta=0$ y $\delta=0,08$.

I No es simple encontrar en un sistema medianamente realista, que presenta todos los armónicos de Fourier, una resonancia simple, aislada. Es claro que siempre existirá alguna intersección entre ellas. Este análisis pretende que tal intersección de nuestra resonancia $1: 1$, que es de bajo orden, no ocurra con ninguna otra de bajo orden, de modo que su ancho resulte despreciable y por consecuencia, a efectos prácticos, no tenga incidencia sobre la resonancia en estudio. 


\subsubsection{Experimento 1}

En esta sección vamos a considerar el desarrollo en torno a $y_{1}^{*}=y_{2}^{*}=\sqrt{3}+\delta$ con $\delta=0$, e intentaremos estimar el orden óptimo de la transformación de manera empírica para tres valores de $\gamma$, dado que el orden óptimo depende, en principio, del parámetro perturbativo: un valor grande $(\gamma=0,1)$ con un nivel elevado de caos, donde se evidencia una fuerte superposición de resonancias, un valor pequeño de $\gamma(\gamma=0,01)$ donde el sistema tiene una componente regular dominante, de manera de considerar dos configuraciones extremas del sistema y, por último, también consideraremos un valor intermedio, $\gamma=0,0298$. Para los dos experimentos que se presentan a continuación, se han escogido $\mathrm{N}_{\mathrm{ci}}=10^{3}$ condiciones iniciales, al igual que en la Sección 4.3. En las Figs. 5.4, 5.6 y 5.8 se muestra (para estos tres valores de $\gamma$ ) el gráfico de la evolución de $\eta^{2}$ en función del número de iteraciones $N_{i t e r}$, para los primeros órdenes del desarrollo, $1 \leqslant N \leqslant 4$.
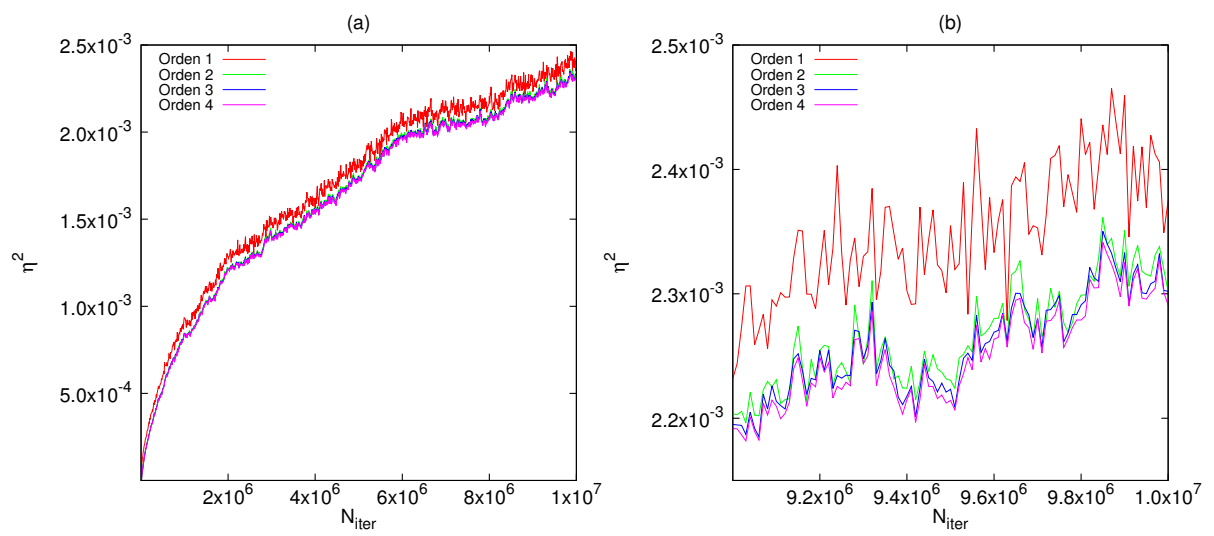

Figura 5.4: (a) Evolución de $\eta^{2}$ con respecto a $N_{i t e r}$ para distintos órdenes $N$, con $1 \leqslant N \leqslant 4, \gamma=0,1$ y $N_{i t e r} \in\left(0 ; 10^{7}\right)$. (b) Acercamiento a la región $N_{i t e r} \in$ $\left(9 \times 10^{6} ; 10^{7}\right)$.

El panel (a) de la Fig. 5.4 muestra la evolución de $\eta^{2}$ para $\gamma=0,1$ en el intervalo $\mathrm{N}_{\text {iter }} \in\left(0 ; 10^{7}\right)$. A excepción de la evolución para $\mathrm{N}=1$ que es ligeramente mayor que el resto, los demás órdenes son muy similares entre sí, resultando imposibles de discriminar en este gráfico. Un acercamiento de la región $9 \times 10^{6} \leqslant N_{\text {iter }} \leqslant 10^{7}$ (Fig. 5.4, panel (b)) nos muestra que existe una diferencia entre $\mathrm{N}=2$ y los órdenes restantes. Esto quiere decir que las curvas correspondientes a $\mathrm{N}=3$ y N $=4$ permanecen sin manifestar diferencias apreciables.

Comparando el mayor de estos órdenes $(\mathrm{N}=4)$ con uno relativamente alto, digamos $N=12$ (Fig. 5.5, panel (a)), podemos ver que presentan una semejanza 
aún mayor a la presentada para los órdenes 3 y 4 . Haciendo una inspección de las últimas $10^{6}$ iteraciones de la evolución $\left(9 \times 10^{6} \leqslant \mathrm{~N}_{\text {iter }} \leqslant 10^{7}\right)$, representadas en el panel (b) de la Fig. 5.5, no se distinguen diferencias en sus magnitudes. Por lo tanto, concluimos que no es posible definir un orden óptimo mediante el análisis realizado hasta orden $\mathrm{N}=12$ de la evolución de la d.c.m., y podemos elegir indistintamente cualquiera de estos órdenes, como haremos más adelante. Veamos qué ocurre al considerar un valor de $\gamma$ más pequeño.
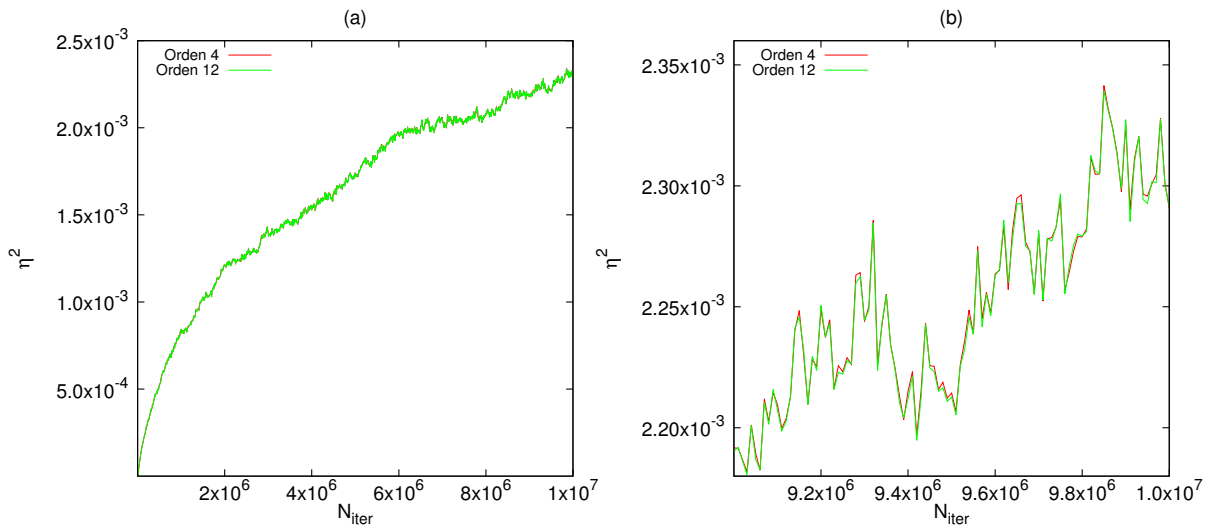

Figura 5.5: (a) Evolución de $\eta^{2}$ con respecto a $N_{\text {iter }}$ para los órdenes $\mathrm{N}=4$ y $N=12$, con $\gamma=0,1$ y $N_{\text {iter }} \in\left(0 ; 10^{7}\right)$. (b) Acercamiento a la región $\mathrm{N}_{\text {iter }} \in(9 \times$ $\left.10^{6} ; 10^{7}\right)$.

La evolución de la d.c.m. con $\gamma=0,0298$ para los órdenes $1 \leqslant N \leqslant 4$ (Fig. 5.6) muestra que es posible distinguir el comportamiento de $\eta^{2}$ para órdenes 1 y 2, pero esto no es posible hacerlo para losórdenes 3 y 4 . Comparando la región $9 \times 10^{6} \leqslant N_{\text {iter }} \leqslant 10^{7}$ ) (Fig. 5.6, panel (b)), con la correspondiente para $\gamma=0,1$ (panel (b) de la Fig. 5.4), vemos que los órdenes 3 y 4 también son muy similares entre sí, y menores que los correspondientes a $\mathrm{N}=1$ y $\mathrm{N}=2$.

$\mathrm{Si}$ inspeccionamos el panel (a) de la Fig. 5.7, que representa la comparación entre las evoluciones para $\mathrm{N}=4$ y para $\mathrm{N}=12$, nuevamente no es posible apreciar diferencias entre ambas gráficas. La Fig. 5.7, panel (b) muestra que, incluso realizando un acercamiento del intervalo $9 \times 10^{6} \leqslant N_{i t e r} \leqslant 10^{7}$ ambas curvas presentan una gran semejanza en el tamaño de las oscilaciones. Con esto debemos concluir que, para tamaños intermedios de $\gamma$, tampoco es posible definir un orden óptimo para la transformación.

Por último, para el valor más pequeño de $\gamma$ considerado $(\gamma=0,01)$ vemos que, en el panel (a) de la Fig. 5.8 el comportamiento es similar al analizado para $\gamma=0,0298$, es decir que es posible distinguir las curvas para $\mathrm{N}=1$ y $N=2$ de aquéllas para órdenes $\mathrm{N}=3$ y $\mathrm{N}=4$, las cuales son muy similares entre 

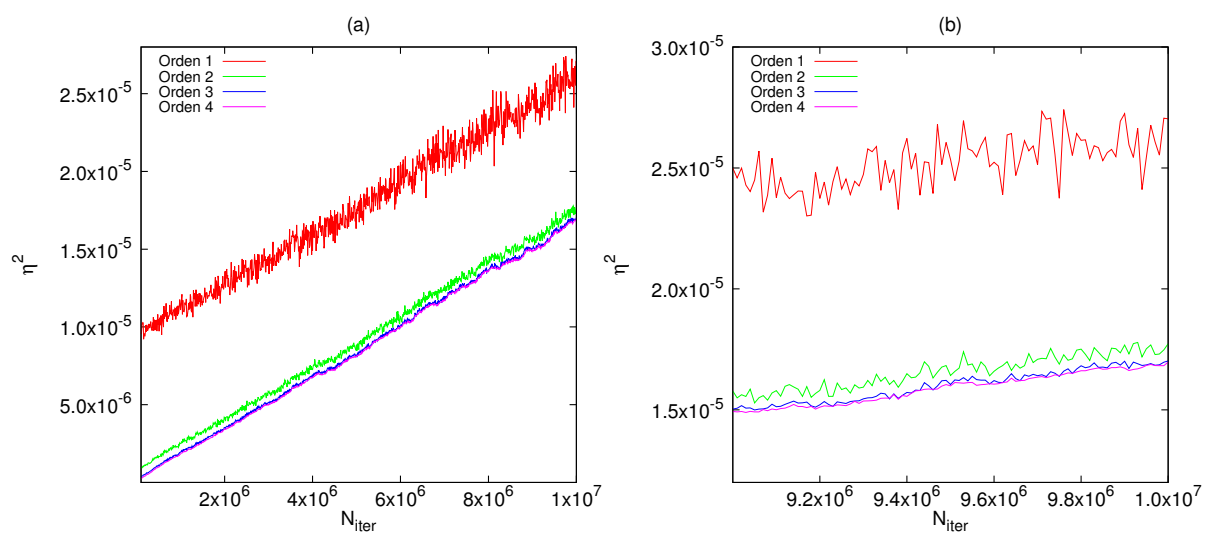

Figura 5.6: (a) Evolución de $\eta^{2}$ con respecto a $N_{\text {iter }}$ para distintos órdenes $N$, con $1 \leqslant N \leqslant 4$ y $\gamma=0,0298$ y $N_{i t e r} \in\left(0 ; 10^{7}\right)$. (b) Acercamiento de la región $\mathrm{N}_{\text {iter }} \in\left(9 \times 10^{6} ; 10^{7}\right)$.
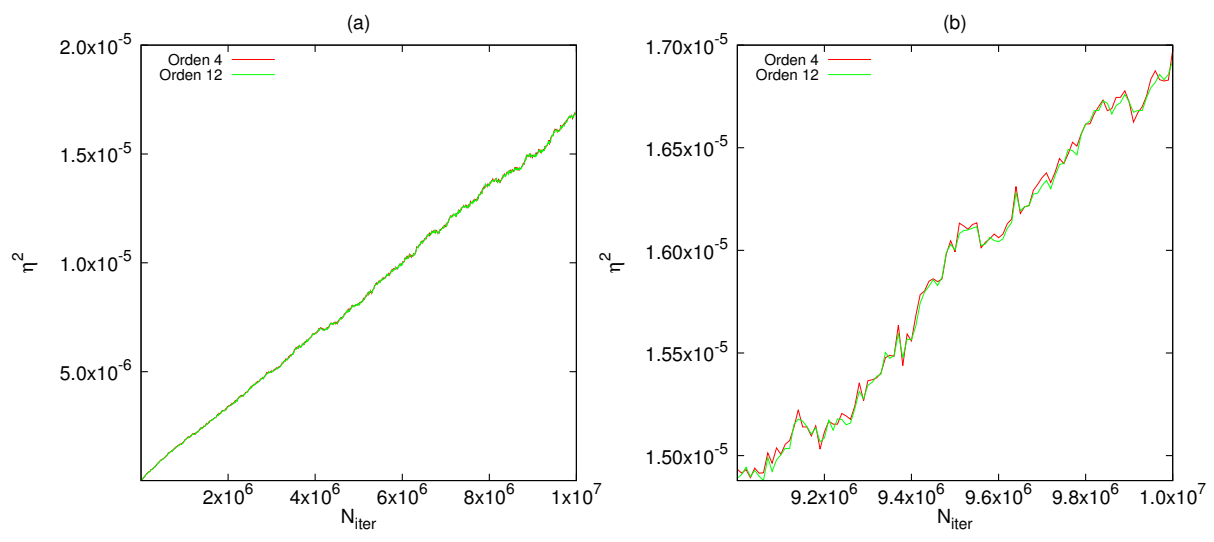

Figura 5.7: (a) Evolución de $\eta^{2}$ con respecto a $N_{i t e r}$ para los órdenes $\mathrm{N}=4$ y $\mathrm{N}=12$, con $\gamma=0,0298$ y $\mathrm{N}_{\text {iter }} \in\left(0 ; 10^{7}\right)$. (b) Acercamiento de la región $\mathrm{N}_{\text {iter }} \in$ $\left(9 \times 10^{6} ; 10^{7}\right)$.

sí. Si reajustamos la escala del eje de las ordenadas, manteniendo el intervalo de tiempo fijo en $\mathrm{N}_{\text {iter }} \in\left(0 ; 10^{7}\right)$ y eliminando de la comparación la curva de orden $\mathrm{N}=1$, es posible apreciar la diferencia entre los órdenes $\mathrm{N}=3$ y $\mathrm{N}=4$. Comparando luego la curva correspondiente a orden 4 con la correspondiente a $\mathrm{N}=12$, resulta (a diferencia de las comparaciones realizadas para $\gamma=0,1$ y $\gamma=0,0298)$ que existe una reducción en el tamaño de las oscilaciones que muestra la curva para $\mathrm{N}=12$, además de presentar un valor promedio menor que para $\mathrm{N}=4$. 

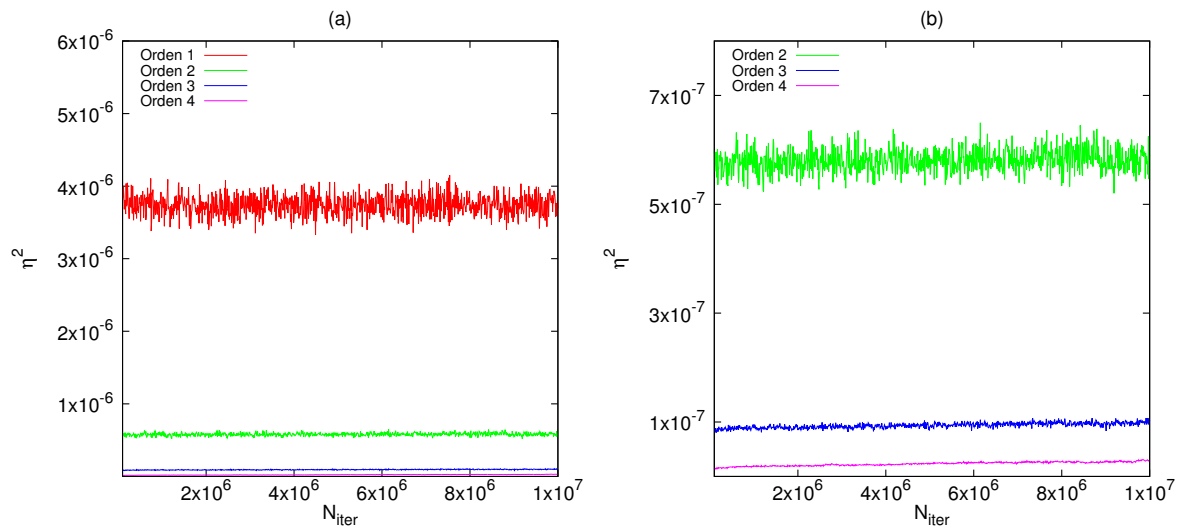

Figura 5.8: (a) Evolución de $\eta^{2}$ con respecto a $N_{\text {iter }}$ para distintos órdenes $N$, con $1 \leqslant N \leqslant 4, \gamma=0,01$ y $N_{i t e r} \in\left(0 ; 10^{7}\right)$. (b) Acercamiento de la región $\mathrm{N}_{\text {iter }} \in\left(9 \times 10^{6} ; 10^{7}\right)$.

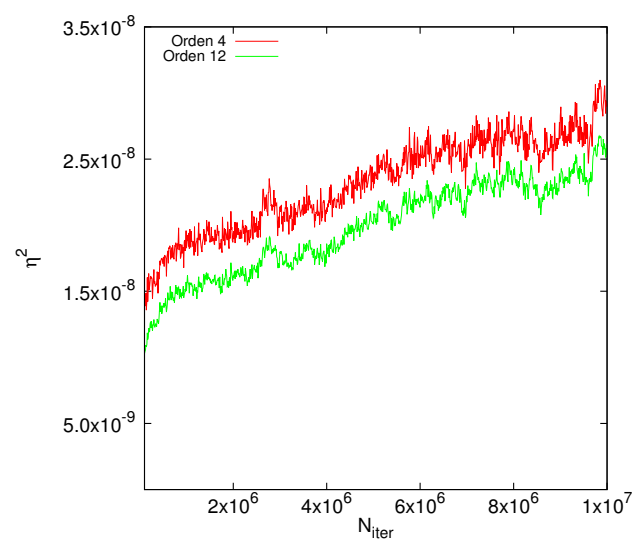

Figura 5.9: Evolución de $\eta^{2}$ con respecto a $N_{\text {iter }}$ para los órdenes $N=4$ y $N=12$, con $\gamma=0,01$ y $N_{\text {iter }} \in\left(0 ; 10^{7}\right)$.

El análisis llevado a cabo en esta sección arrojó un resultado interesante: para valores grandes de $\gamma(0,0298 \leqslant \gamma \leqslant 0,1)$ no se pudo encontrar empíricamente, y mediante este análisis, un orden óptimo para el desarrollo. Para $\gamma=0,01$, en cambio, el comportamiento observado fue distinto. Las curvas que representan la evolución de la d.c.m. mostraron una reducción progresiva en sus oscilaciones a medida que incrementamos el orden del desarrollo. Sin embargo, tampoco ha sido posible estimar un orden óptimo, a partir del cual las oscilaciones vuelvan a aumentar. Es probable que, en este caso, el orden óptimo sea $r_{\text {opt }}>12$ pero, debido a que los tiempos de CPU aumentan considerablemente 
para órdenes altos $(N \geqslant 9$, ver Sección 5.2.1), no se ha avanzado en la búsqueda del orden óptimo para $y_{1}^{*}=y_{2}^{*}=\sqrt{3}$ y $\gamma=0,01$.

\subsubsection{Experimento 2}

En la Sección 5.2.2.1 hemos visto que, si elegimos el punto central $\left(y_{1}^{*}, y_{2}^{*}\right)$ de manera que se encuentre en una resonancia simple, pero está muy cerca de una región de superposición con resonancias de alto orden producidas por la proximidad con un cruce de resonancias, no es posible poder determinar un orden óptimo.

En esta sección estudiaremos el comportamiento de las oscilaciones en la evolución de la d.c.m. para distintos órdenes $\mathrm{N}$, eligiendo el punto central de manera tal que se encuentre en una resonancia simple, pero alejada de un cruce de resonancias. Así, la dinámica del conjunto de valores iniciales no se vería afectada por la estructura compleja de un cruce de resonancias, como fue el caso para $\delta=0$. Una vez elegido este punto, el desarrollo de la transformación para una resonancia simple es válido, y podemos emplearlo en nuestro intento de estimar empíricamente el orden óptimo $\mathrm{r}_{\mathrm{opt}}$.

Basándonos en los experimentos realizados en la subsección anterior, elegiremos los mismos valores de $\gamma$ para estimar el orden óptimo.

Para $\gamma=0,1$, la similitud que presentan las curvas de evolución para todos los órdenes calculados en el rango de tiempos $\mathrm{N}_{\text {iter }} \in\left(0 ; 10^{7}\right)$ y mostrados en el panel (a) de la Fig. 5.1o (los órdenes $\mathrm{N}>9$ se han omitido en el gráfico por tener oscilaciones con tamaños mucho mayores que las mostradas), hace que resulte imposible distinguir diferencias en éstas. Esto hizo necesaria la construcción de un nuevo gráfico (Fig. 5.10, panel (b)) donde se magnifica la región de las últimas $10^{6}$ iteraciones. Se eliminaron también los órdenes extremos $\mathrm{N}=4 \mathrm{y}$ $\mathrm{N}=9$ porque, al igual que con $\mathrm{N}>9$, sus oscilaciones son mucho mayores en comparación con el resto. En cuanto a los órdenes restantes $(5 \leqslant N \leqslant 8)$, todas las curvas exhiben oscilaciones cuyas amplitudes son aproximadamente del mismo tamaño. Por lo tanto, se infiere que el orden óptimo se encontrará en $5 \leqslant r_{\text {opt }} \leqslant 8$.

Si analizamos ahora las curvas correspondientes a $\gamma=0,0298$, éstas presentan diferencias en las magnitudes de sus oscilaciones en el intervalo $N_{i t e r} \in\left(0 ; 10^{7}\right)$ (Fig. 5.11, panel (a)). Analizando y comparando las curvas del gráfico mencionado, vemos que la evolución correspondiente a $\mathrm{N}=9$ posee, claramente, mayores oscilaciones que el resto. Por otro lado, aunque la diferencia es menor, la evolución para $\mathrm{N}=4$ también muestra que posee oscilaciones con amplitudes ligeramente mayores que las evoluciones para $5 \leqslant N \leqslant 8$. Una vez descartados estos dos valores de $\mathrm{N}$, construimos nuevamente un gráfico de la evolución de 

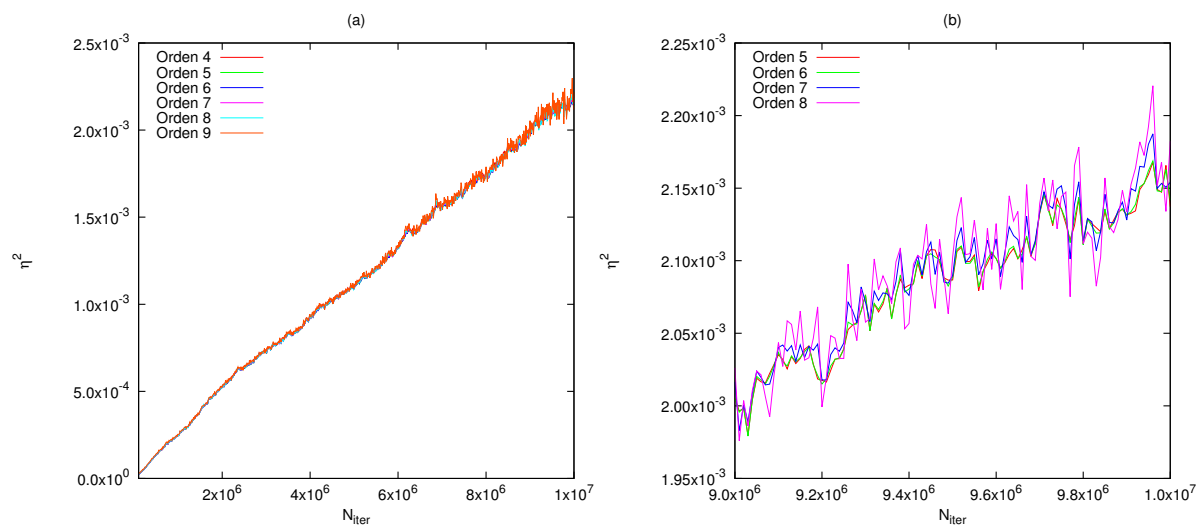

Figura 5.10: (a) Evolución de $\eta^{2}$ con respecto a $N_{\text {iter }}$ para distintos órdenes de magnitud $N$, con $4 \leqslant N \leqslant 9$, para $\gamma=0,1$ y $N_{\text {iter }} \in\left(0 ; 10^{7}\right)$. (b) Acercamiento de la región $\mathrm{N}_{\text {iter }} \in\left(9 \times 10^{6} ; 10^{7}\right)$.

$\eta^{2}$ con respecto al orden $N$, focalizándonos en la región $N_{\text {iter }} \in\left(9 \times 10^{6} ; 10^{7}\right)$, obteniendo el panel (b) de la Fig. 5.11. Con este gráfico podemos confirmar lo que ya se ve en el panel (a), es decir, que el tamaño en las oscilaciones para $5 \leqslant N \leqslant 8$ es comparable. En principio, cualquiera de estos valores de $N$ que define cada curva podría ser adoptado como orden óptimo, debido a la similitud en el tamaño de las amplitudes de sus oscilaciones. Para definir cuál de estos cuatro valores será elegido como $r_{o p t}$ podemos considerar el valor promedio de la curva, sin tener en cuenta las oscilaciones. Haciendo esto en el panel (b) de la Fig. 5.11, se observa que la evolución correspondiente a $\mathrm{N}=7$ se encuentra por debajo de todas las demás, presentando un valor promedio menor. En base a este resultado, adoptamos como óptimo el valor $r_{o p t}=7$.

Para $\gamma=0,1$ también nos encontramos en una situación en la que las curvas para $5 \leqslant N \leqslant 8$ manifestaron oscilaciones de tamaños comparables, mientras que las oscilaciones de los órdenes superiores fueron muchos mayores. Esto hizo que no se decidiera un criterio para la elección del orden óptimo. Por ende, adoptamos $r_{\mathrm{opt}}=7$ como orden óptimo, de manera de realizar todos los experimentos con el mismo orden en la transformación.

Consideremos por último la evolución correspondiente a $\gamma=0,01$ (Fig. 5.12, panel (a)), donde todas las curvas presentan oscilaciones con grandes amplitudes. Sin embargo, si tenemos en cuenta el valor promedio de éstas, se destacan del resto las correspondientes a $\mathrm{N}=4$ y $\mathrm{N}=7$, que son las dos de menor valor (representadas por las líneas de color rojo y violeta, respectivamente). La Fig. 5.12, panel (b), permite confirmar que el tamaño en las oscilaciones de las curvas es comparable, por lo que el tamaño de las oscilaciones no puede ser utilizado como criterio de selección del $r_{\mathrm{opt}}$. Esta conclusión puede tener origen 

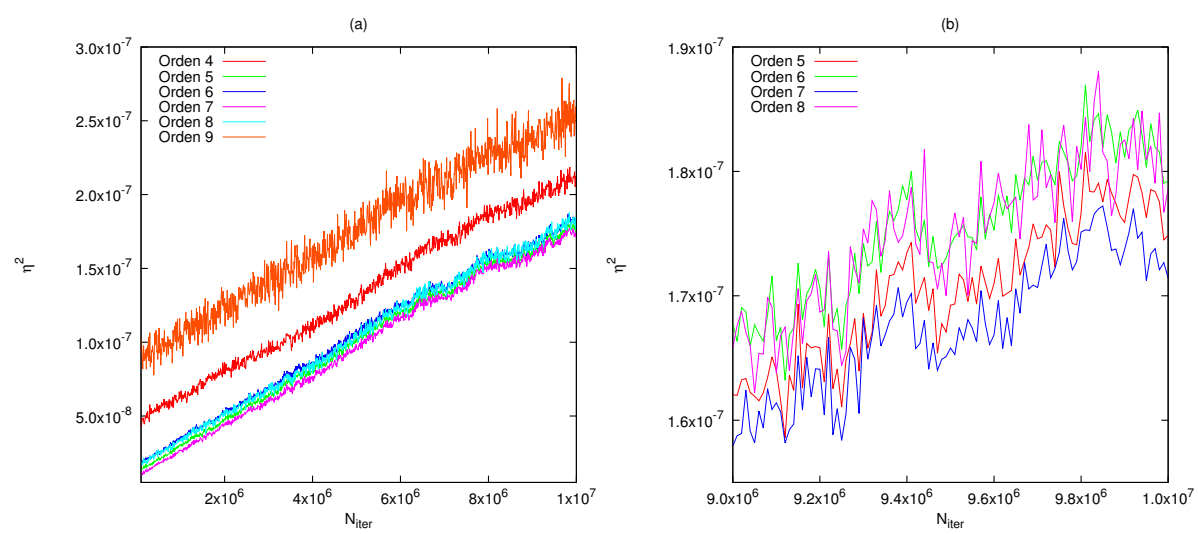

Figura 5.11: (a) Evolución de $\eta^{2}$ con respecto a $N_{\text {iter }}$ para distintos órdenes de magnitud $N$, con $4 \leqslant N \leqslant 9$, para $\gamma=0,0298$ y $N_{i t e r} \in\left(0 ; 10^{7}\right)$. (b) Acercamiento de la región $\mathrm{N}_{\text {iter }} \in\left(9 \times 10^{6} ; 10^{7}\right)$.

en el hecho de que, al ser $\gamma$ un parámetro tan pequeño, la variación secular de la d.c.m. también es muy pequeña y, la disminución de la amplitud de las oscilaciones a causa de la implementación de la transformación, no es suficiente como para que se logre evidenciar la d.c.m.
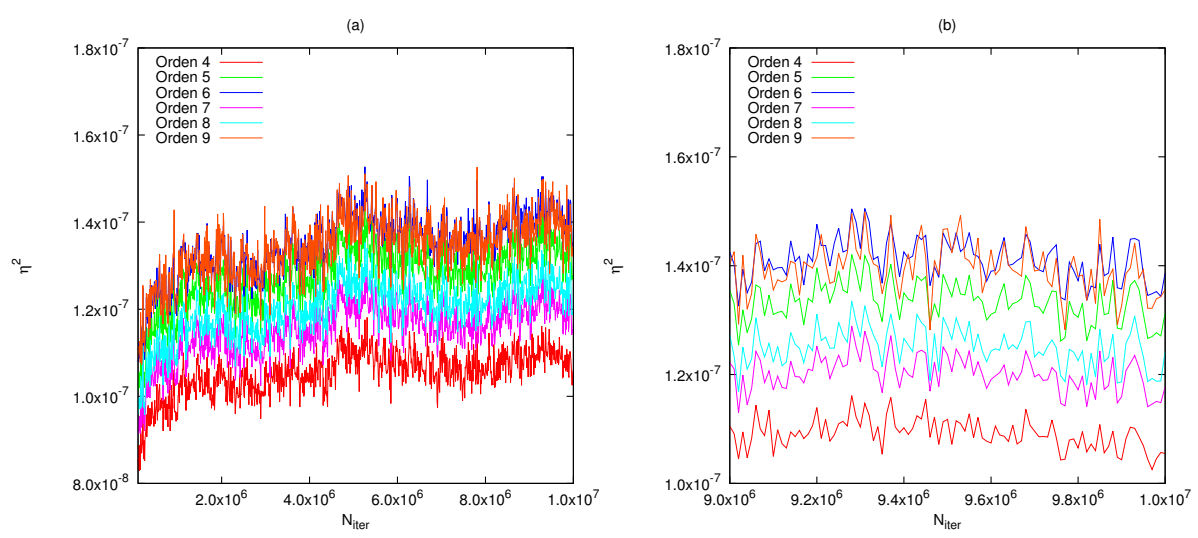

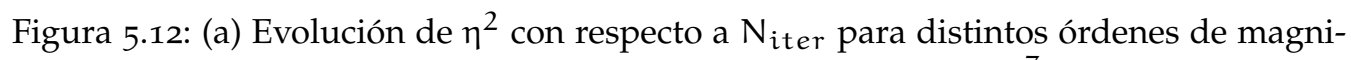
tud $N$, con $4 \leqslant N \leqslant 9$, para $\gamma=0,01$ y $N_{\text {iter }} \in\left(0 ; 10^{7}\right)$. (b) Acercamiento de la región $\mathrm{N}_{\text {iter }} \in\left(9 \times 10^{6} ; 10^{7}\right)$.

En base al análisis realizado para este valor de $\gamma$, tendríamos que $\mathrm{N}=4$ sería el candidato a considerar como nuestro orden óptimo, dado que es su curva correspondiente es la que presentó un valor promedio menor. Pero, dado que para $\mathrm{N}=7$ también obtuvimos un valor promedio pequeño, el incremento del error por adoptar este valor es despreciable $y$, si bien su $t_{C P U}$ es varias veces 
mayor que aquel para $\mathrm{N}=4$, numéricamente este tiempo es una cantidad pequeña. Por ende adoptamos el valor $r_{o p t}=7$, para mantener una uniformidad en la elección del orden óptimo.

\section{$5 \cdot 3$ DISCUSIÓN}

En este capítulo se mostró, en una manera detallada, cómo aplicar el desarrollo de la transformación a formas normales al mapa CRSSM. Se fijaron todos sus parámetros excepto dos de ellos, $\gamma$. Luego se escogieron algunos valores de $\gamma$ que cubren el rango $0,01 \leqslant \gamma \leqslant 0,1$, de manera de contemplar desde escenarios donde existe una fuerte superposición de resonancias, hasta aquéllos en donde las resonancias de más bajo orden son levemente visibles.

Se presentó un programa, escrito en Fortran 90, formado por tres subprogramas para el cálculo de la transformación, la forma normal y la transformación inversa. El programa también construye la serie de tiempos en las variables transformadas y calcula la variación de las acciones con respecto a su valor inicial (d.c.m.) en ambos conjuntos de variables, en la dirección de la resonancia $\left(I_{f}\right)$ y en la dirección perpendicular a ella $\left(I_{R}\right)$.

Para probar la eficiencia de la implementación realizada, se midieron los tiempos de cómputo. Se estimó una dependencia entre el tiempo de CPU empleado por cada uno de estos programas y el orden de la transformación.

En la última parte de este capítulo se construyó la transformación alrededor de dos puntos pertenecientes a la resonancia $1: 1$, en el espacio de las acciones, $y_{1}^{*}=y_{2}^{*}=\sqrt{3}+\delta$, para $\delta=0$ y $\delta=0,08$. Se calculó la d.c.m. para dos conjuntos de $\mathrm{N}_{\mathrm{ci}}=10^{3}$ condiciones iniciales. Con estos resultados se intentó estimar de forma empírica el orden óptimo $r_{o p t}$ de la transformación a través del cálculo de la d.c.m. para órdenes desde $\mathrm{N}=4$ hasta $\mathrm{N}=12$. Para el punto correspondiente a $\delta=0,08$ se se adoptoó como el orden del desarrollo que minimizó las oscilaciones en las curvas de d.c.m. al valor $r_{o p t}=7$, el cual se utilizará como orden del desarrollo para los cálculos que realizaremos en el capítulo siguiente. En cuanto al punto correspondiente a $\delta=0$, la cercanía con un cruce de resonancias y las superposiciones, hicieron que las oscilaciones producidas nos impidan observar el cambio de tendencia de la evolución de la d.c.m., haciendo imposible determinar un orden óptimo. Se adoptó como $r_{o p t}$ para este escenario $r_{\mathrm{opt}}=7$ para mantener una uniformidad con el valor escogido para $\delta=0,08$. Debido a que las oscilaciones en las curvas de d.c.m. para los distintos órdenes resultaron muy similares, el error esperable por el cálculo de la difusión al tomar este valor de $r_{\mathrm{opt}}$ es muy pequeño. 


\section{6}

\section{CUANTIFICACIÓN DE LA DIFUSIÓN}

En el Capítulo 4 hemos presentado un procedimiento para construir una transformación canónica a un mapa simpléctico 4D cuasi-torsional (las formas normales) que nos permite describir la dinámica del sistema de una manera más simple. Luego, en el Capítulo 5, hemos mostrado cómo implementar este procedimiento a un mapa en particular, el CRSSM.

El objetivo de este capítulo es la caracterización de la difusión en las vecindades de un punto central $\left(y_{1}^{*}, y_{2}^{*}\right)$ en un escenario de caos débil ${ }^{1}$. Este escenario nos permitirá suponer que el comportamiento de la difusión obedece una ley de potencias ([18], [21], [22], [60]), de lo contrario, si considerásemos un escenario de caos fuerte, la difusión dependería linealmente del tiempo (o, en el caso discreto, del número de iteraciones). Luego, a través del análisis de la evolución de la d.c.m., intentaremos identificar qué tipo de difusión se presenta para las distintas configuraciones (esto es, subdifusión, superdifusión o difusión normal, como se definió en la Sección 4.1.1). Una vez caracterizada la difusión en función del exponente de la ley de potencias que obedezca, para aquellos escenarios en los que se puede caracterizar como difusión normal, estimaremos el coeficiente de difusión $\mathcal{D}$.

\subsection{PREPARACIÓN DEL ENTORNO PARA EL ESTUDIO DE LA DIFUSIÓN}

A efectos de cuantificar la difusión en el espacio de acciones utilizaremos como herramienta la desviación cuadrática media, descripta en la Sección 4.1.1.

Los resultados que se presentarán en este capítulo corresponden a los mismos parámetros que se han utilizado en la Sección 4.2. El único parámetro que ha quedado libre es el de acoplamiento $\gamma$, que tomará valores en un rango de un orden de magnitud $(0,01 \leqslant \gamma \leqslant 0,1)$. Para seleccionar estos valores de $\gamma$ se realizaron varios experimentos variando esta cantidad. Los valores extremos fueron escogidos de manera de obtener escenarios en los que la manifestación de una evolución secular de las acciones es notoria, como también aquéllos en

1 Llamamos un escenario de caos débil a aquél en el que la perturbación es lo suficientemente pequeña como para que se pueda despreciar cualquier efecto de superposición de resonancias. 
los que ésta es tan lenta que debemos recurrir a las técnicas descriptas en el Capítulo 4 . Se han escogido 20 valores diferentes de $\gamma$, todos ellos equiespaciados en una escala logarítmica, para conseguir un amplio espectro de escenarios.

Nos enfocaremos en la misma resonancia considerada en el Capítulo 4, la 1 : 1. En esta resonancia hemos escogido las frecuencias $\sqrt{3}+\delta$, tal como lo hemos hecho en la Sección 5.1.

Como este análisis será realizado utilizando el cálculo de la forma normal, y éste es válido solamente en una resonancia simple, necesitamos que el punto central esté suficientemente lejos de cualquier cruce de resonancias. Siguiendo el análisis de la Sección 5.2.2.2, adoptamos el valor $\delta=0,08$ (ver Fig. 5.3), obteniendo $y_{1}^{*}=y_{2}^{*} \approx 1,812050808$, de manera que el punto $\left(y_{1}^{*}, y_{2}^{*}\right)$ se encuentre "lejos "de un cruce de resonancias.

\subsubsection{Condiciones iniciales}

El análisis de la difusión realizado en este capítulo se llevó a cabo considerando la evolución de un conjunto de $\mathrm{N}_{\mathrm{ci}}=10^{3}$ condiciones iniciales. Dado que nuestro objetivo es estudiar la variación secular de las acciones en la dirección de la resonancia, y queremos que $\eta^{2}\left(N_{i t e r}=0\right)=0$, es decir, que inicialmente no exista variación, las condiciones iniciales deben estar todas ubicadas sobre un mismo segmento de recta en dirección perpendicular al sentido de la resonancia. Luego, se las distribuye sobre dicho segmento, que será de longitud $\delta \rho \sim 10^{-9}$, de manera que se encuentren todas en la región de la capa estocástica donde el caos es más uniforme, reduciendo la probabilidad de que alguno de estos valores estén dentro de una isla de estabilidad.

El cálculo se realizó para un total de $\mathrm{N}_{\text {iter }}=10^{7}$ iteraciones, con un paso de escritura de $10^{4}$ iteraciones, obteniendo una serie temporal de $10^{3}$ puntos.

Para corroborar que las condiciones iniciales están en una región caótica, la Fig. 6.I muestra, en el panel (a), el valor final del $\ln (F L I)$ en función del número de condición inicial del conjunto, para $10^{4}$ iteraciones. Este cálculo, efectuado con el LP-VIcode, tiene establecido un valor de saturación para el FLI, que es $F L I_{\text {sat }}=10^{20}$, de donde $\ln \left(\mathrm{FLI}_{\text {sat }}\right) \approx 46,05$. Podemos ver que la totalidad de las condiciones iniciales del conjunto han alcanzado el valor de saturación. Vemos reflejado este resultado en el panel (b) de la Fig. 6.I, donde se muestra el tiempo que le llevó a cada condición inicial llegar al valor de saturación del FLI, siendo todos menores que $10^{4}$. Esto ocurre debido a que cada condición inicial que forma parte del ensamble se encuentra dentro de una región caótica, esto es, la capa estocástica de la resonancia. 

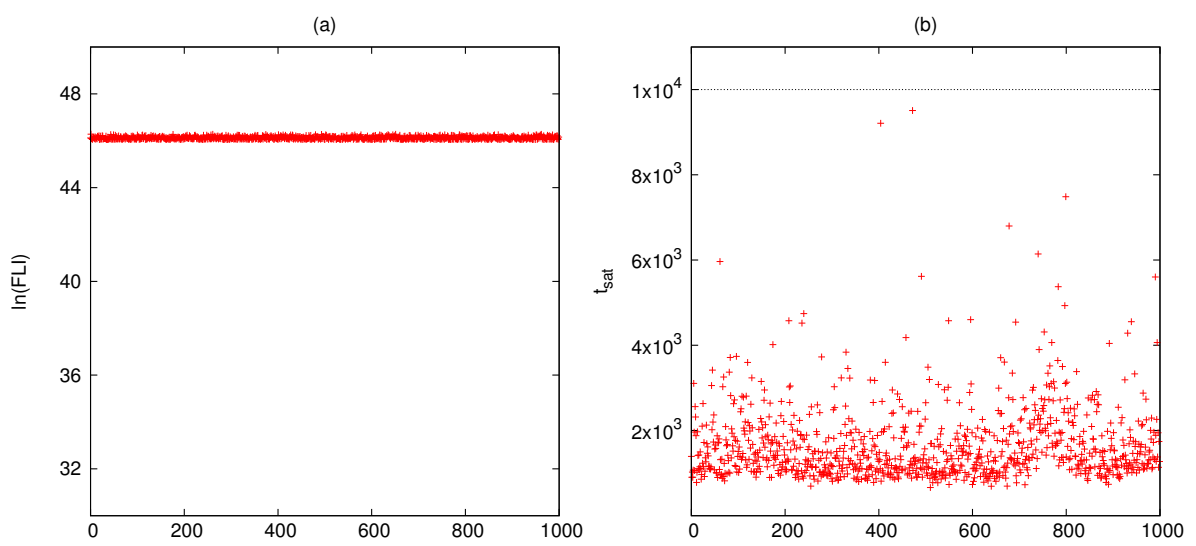

Figura 6.1: (a) Valor final del $\ln (\mathrm{FLI})$ para $10^{4}$ iteraciones, con un valor de saturación de $\ln \left(10^{20}\right)$, en las vecindades de $\left(y_{1}^{*}, y_{2}^{*}\right)=(\sqrt{3}+\delta, \sqrt{3}+\delta)$ con $\delta=0,08$. El eje de las abscisas corresponde al número de condición inicial del conjunto. (b) Tiempo de saturación del FLI para las mismas condiciones que en (a). La línea punteada representa el tiempo de cálculo, $\mathrm{N}_{\mathrm{iter}}=10^{4}$.
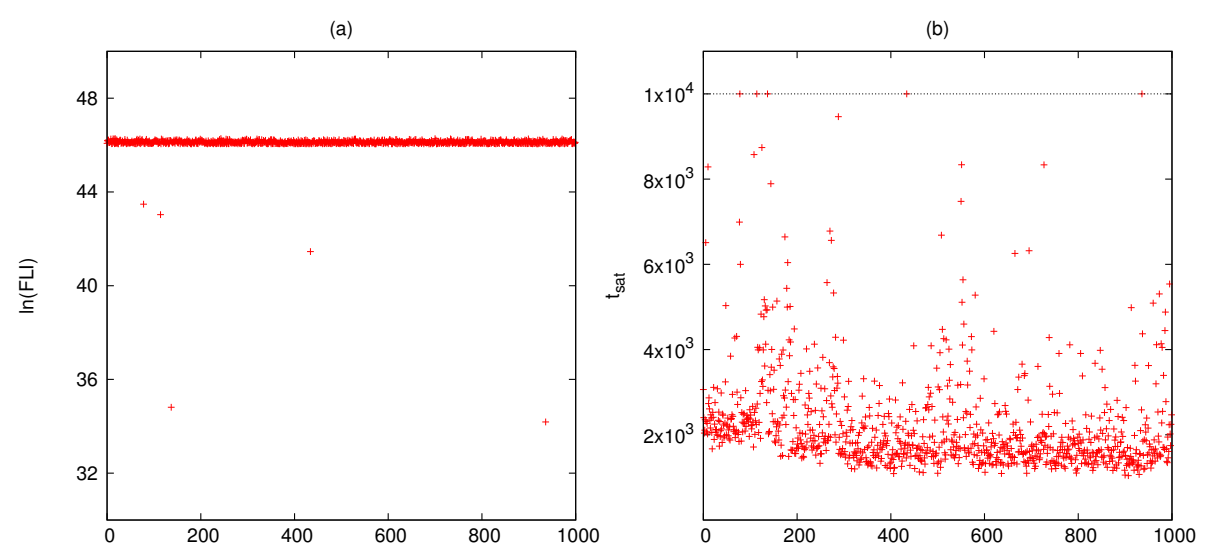

Figura 6.2: (a) Valor final del $\ln (\mathrm{FLI})$ para $10^{4}$ iteraciones, con un valor de saturación de $\ln \left(10^{20}\right)$, en las vecindades de $\left(y_{1}^{*}, y_{2}^{*}\right)=(\sqrt{3}+\delta, \sqrt{3}+\delta)$ con $\delta=0$. El eje de las abscisas corresponde al número de condición inicial del conjunto. (b) Tiempo de saturación del FLI para las mismas condiciones que en (a). La línea punteada representa el tiempo de cálculo, $\mathrm{N}_{\text {iter }}=10^{4}$.

En cuanto a las condiciones iniciales que se estudiarán en la Sección 6.4, correspondientes al punto central $y_{1}^{*}=y_{2}^{*}=\sqrt{3}+\delta$, con $\delta=0$, y desplegadas en el panel (a) de la Fig. 6.2, los valores del FLI de las condiciones iniciales han saturado casi en su totalidad, quedando solamente 5 condiciones iniciales que han llegado a $\mathrm{N}_{\text {iter }}=10^{4}$ sin saturar (hecho que se ve en la Fig. 6.2, panel (b)). Sin embargo, vemos que para la condición inicial que tomó el mínimo 
valor, hemos obtenido $\ln (\mathrm{FLI}) \approx 34,18$, lo que corresponde a un valor del FLI $\approx$ $7 \times 10^{14}$. Recordemos que para que una órbita pueda ser considerada como regular, el valor del FLI debe comportarse como FLI $\propto N_{i t e r}$. Basándonos en este concepto hemos analizado una muestra de órbitas regulares, resultando para todas ellas que FLI $\sim N_{\text {iter. }}$ Por lo tanto, para $N_{\text {iter }}=10^{4}$, un valor de saturación del FLI de $10^{20}$ es suficiente para poder clasificar correctamente las órbitas regulares y caóticas. Con este resultado mostramos que, incluso las condiciones iniciales cuyo FLI no saturó, también son órbitas caóticas, ya que su valor es $\sim 10$ órdenes de magnitud mayor que el umbral considerado para una órbita regular.

\subsection{GRÁFICOS COMPARATIVOS PRE Y POST-TRANSFORMACIÓN}

Esta sección será dedicada a mostrar cómo la transformación a formas normales mejora la claridad con que se representa la variación secular de las acciones. Esto se logrará mostrando gráficos de la evolución de la d.c.m. en el conjunto nuevo de variables y comparándolas con la evolución de la d.c.m. calculada a partir de las variables originales del sistema.

Como el proceso de la transformación a formas normales es costoso computacionalmente, también incorporaremos a esta comparación el cálculo de la d.c.m. a través de la selección de los puntos, en el conjunto original de variables, que pertenecen a la región $\left|x_{1}\right|+\left|x_{2}\right| \leqslant 0,1$, como hemos visto en la Sección 4.3, la cual es una técnica que requiere menor esfuerzo computacional.

Una vez establecidos los parámetros que definen el sistema (Sección 4.2), estimado empíricamente el orden óptimo $r_{o p t}$ para realizar la transformación (Sección 5.2.2), y escogidas las condiciones iniciales (Sección 6.1.1), procedemos a realizar los cálculos y hacer las comparaciones. Dichos cálculos fueron realizados adoptando $\delta=0,08$.

En la Fig. 6.3 se muestra la evolución de la d.c.m. para $\gamma=0,1$. La curva correspondiente al cálculo realizado en el conjunto original de variables presenta oscilaciones de mayor amplitud que la curva construida calculando las formas normales. Sin embargo, es trivial concluir a partir del gráfico, que en ambos conjuntos de variables las curvas exhiben un crecimiento sistemático muy similar. De esta manera, como la d.c.m. crece prácticamente en forma lineal, es posible medir la difusión utilizando cualquiera de los dos conjuntos de variables.

Para la evolución calculada con $\gamma=0,0785$ (Fig. 6.4), se observa un escenario análogo al obtenido para $\gamma=0,1$, donde nuevamente el crecimiento de la d.c.m. puede ser detectado en las variables originales. El tamaño de las amplitudes de las oscilaciones que presenta la curva en las variables originales es similar a 


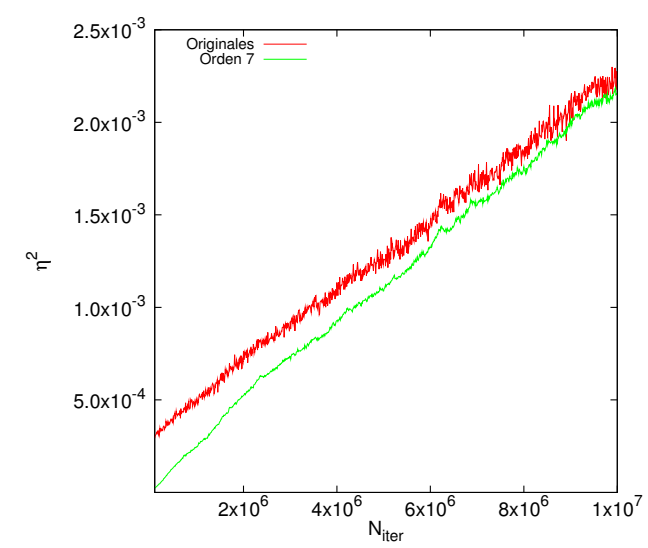

Figura 6.3: Evolución de la d.c.m. para $\gamma=0,1$ en las variables originales $\left(J_{1}, J_{2}, x_{1}, x_{2}\right)$ (curva superior), y luego de una transformación a la forma normal de orden $7\left(\Lambda_{1}, \Lambda_{2}, \theta_{1}, \theta_{2}\right)$ (curva inferior).

las representadas en la Fig. 6.3. Si bien la diferencia entre los valores promedio de las curvas para ambos conjuntos de variables en este escenario es mayor que en el caso de $\gamma=0,1$, solamente nos interesa la velocidad con la que crece dicha curva, cuantificada a través de su pendiente, que es aproximadamente la misma en ambos conjuntos de variables.

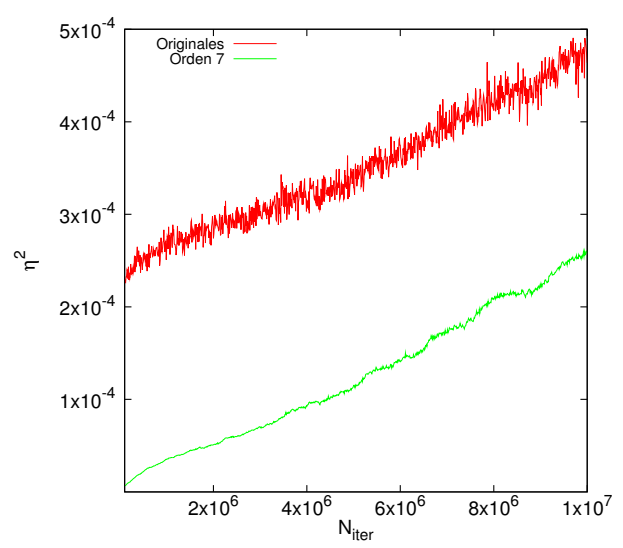

Figura 6.4: Evolución de la d.c.m. para $\gamma=0,0785$ en las variables originales $\left(\mathrm{J}_{1}, \mathrm{~J}_{2}, \mathrm{x}_{1}, \mathrm{x}_{2}\right)$ (curva superior), y luego de una transformación a la forma normal de orden $7\left(\Lambda_{1}, \Lambda_{2}, \theta_{1}, \theta_{2}\right)$ (curva inferior).

Para un parámetro un poco menor, $\gamma=0,0616$ (Fig. 6.5, panel (a)), la evolución también muestra una variación secular de la d.c.m., pero las oscilaciones que ahora presenta hacen que la caracterización de la difusión sea más difícil que en los escenarios anteriores. Esto es debido a que, al intentar realizar dicha 
caracterización por medio del ajuste de una ley de potencias, dicho ajuste se va a llevar a cabo con una gran indeterminación a causa de la amplitud de las oscilaciones, donde los efectos de deformación ${ }^{2}$ se vuelven más importantes. Por ende debemos utilizar las técnicas presentadas en el Capítulo 4 para poder reducir las amplitudes de las oscilaciones.
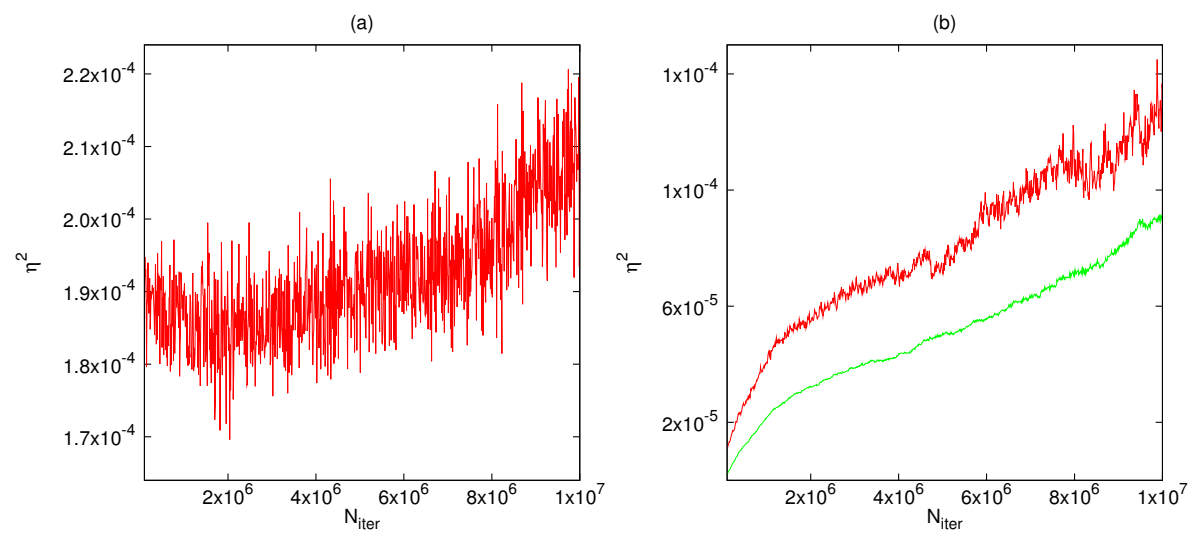

Figura 6.5: (a) Evolución de la d.c.m. con respecto al número de iteraciones $\mathrm{N}_{\text {iter }}$ para $\gamma=0,0616$ en las variables originales $\left(\mathrm{J}_{1}, \mathrm{~J}_{2}, \mathrm{x}_{1}, \mathrm{x}_{2}\right)$. (b) Ídem (a), considerando solamente los puntos dentro de la región $\left|x_{1}\right|+\left|x_{2}\right| \leqslant \delta$, con $\delta=0,1$ (trazo rojo), y luego de una transformación a formas normales de orden $\mathcal{O}(7)\left(\Lambda_{1}, \Lambda_{2}, \theta_{1}, \theta_{2}\right)$ (trazo verde).

En el panel (b) de la Fig. 6.5 se representa la evolución de la d.c.m. calculada considerando ambas técnicas, representando con trazo rojo aquélla que emplea los puntos pertenecientes a la región cercana a la superficie de sección $\left|x_{1}\right|+$ $\left|x_{2}\right| \leqslant 0,1$, y con trazo verde la correspondiente a la aplicación de las formas normales. Ambas curvas manifiestan un crecimiento secular de la d.c.m. con una tendencia similar a la evolución presentada en el panel (a), salvo que esta vez la amplitud de las oscilaciones ha sido notablemente reducida.

Cabe notar que, si bien la técnica que utiliza los puntos cercanos a la superficie de sección es computacionalmente menos costosa, la reducción de la amplitud de las oscilaciones lograda mediante las formas normales es casi total, permitiendo obtener una curva de evolución de la d.c.m. más clara. Esto tiene como consecuencia que la estimación de cualquier valor a partir de esta curva (por ejemplo, el coeficiente de difusión $\mathcal{D}$ en caso de presentar difusión normal) se logre con un nivel de precisión mucho mayor.

2 Se llama efecto de deformación a las oscilaciones producidas por todos los términos del mapa que, mediante sucesivas transformaciones canónicas, pueden suprimirse, es decir, que son los términos que no pertenecen puramente a la perturbación. Para mayor información referirse a [41] 
Si consideramos ahora un valor de $\gamma$ menor, por ejemplo, $\gamma=0,0545$, el tamaño de las amplitudes en las oscilaciones resulta mayor que el crecimiento de la variación secular de la d.c.m., haciendo muy difícil la detección de la difusión (Fig. 6.6, panel (a)). Sin embargo, aunque en este escenario no sea posible detectarla, dicha variación secular existe. Más aún, al calcular la evolución de la d.c.m. utilizando las dos técnicas empleadas para $\gamma=0,0616$, es posible detectar este comportamiento. Este resultado se encuentra representado en el panel (b) de la Fig. 6.6 respetando el mismo código de color que el panel (b) de la Fig. 6.5. Al igual que sucedió para ese valor de $\gamma$, la curva calculada a partir de la aplicación de las formas normales presenta una reducción casi total de las oscilaciones, resultando una técnica muy conveniente cuando se debe priorizar la precisión frente al tiempo de cómputo.
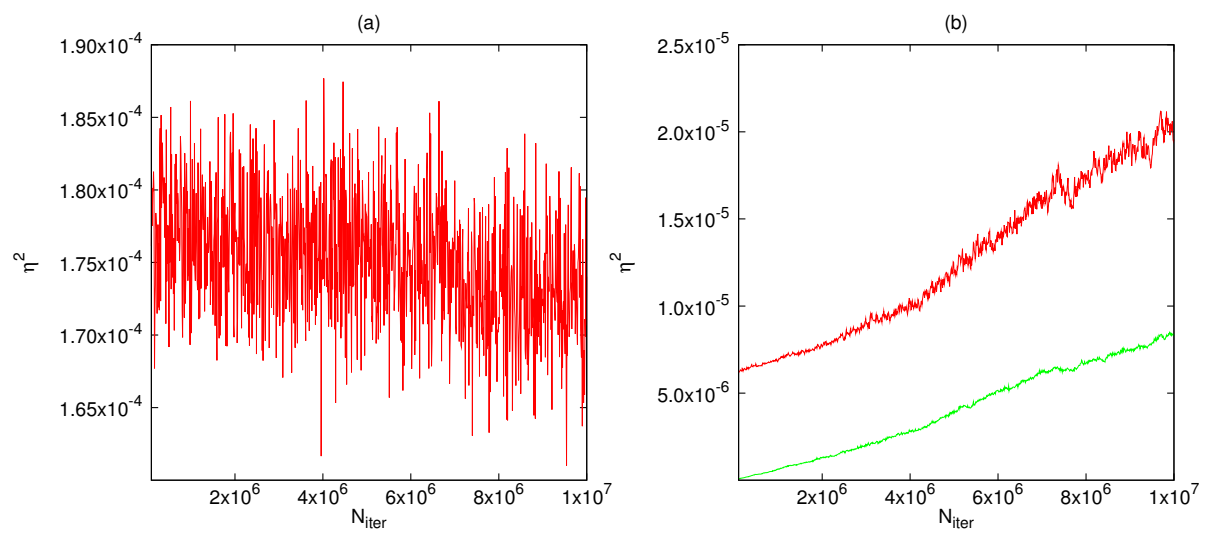

Figura 6.6: Evolución de la d.c.m. para $\gamma=0,0545$ (a) en las variables originales $\left(\mathrm{J}_{1}, \mathrm{~J}_{2}, \mathrm{x}_{1}, \mathrm{x}_{2}\right)$ y $(\mathrm{b})$ luego de una transformación a la forma normal de orden $7\left(\Lambda_{1}, \Lambda_{2}, \theta_{1}, \theta_{2}\right)$ (trazo rojo). En (b) también se incluye la curva obtenida considerando solamente los puntos dentro de la región $\left|x_{1}\right|+\left|x_{2}\right| \leqslant \delta$, con $\delta=0,1$ (trazo verde).

Por ende, de la comparación de las curvas del panel (b) con la correspondiente del panel (a), es evidente la necesidad de aplicar una técnica que reduzca las oscilaciones para valores de $\gamma \leqslant 0,0545$.

La evolución de la d.c.m. en las variables originales para $\gamma=0,0233$ se exhibe en el panel (a) de la Fig. 6.7. Como mencionamos en el párrafo anterior, al ser $\gamma<0,0545$, esta gráfica no revela ningún comportamiento difusivo, por lo que debemos recurrir nuevamente a la utilización de alguna de las dos técnicas utilizadas en los casos previos.

Si construimos la curva de evolución de la d.c.m. considerando los puntos de la región $\left|x_{1}\right|+\left|x_{2}\right| \leqslant 0,1$ es posible ver que, si bien ésta presenta una tendencia secular, el tamaño de la amplitud de las oscilaciones ha sido reducido leve- 

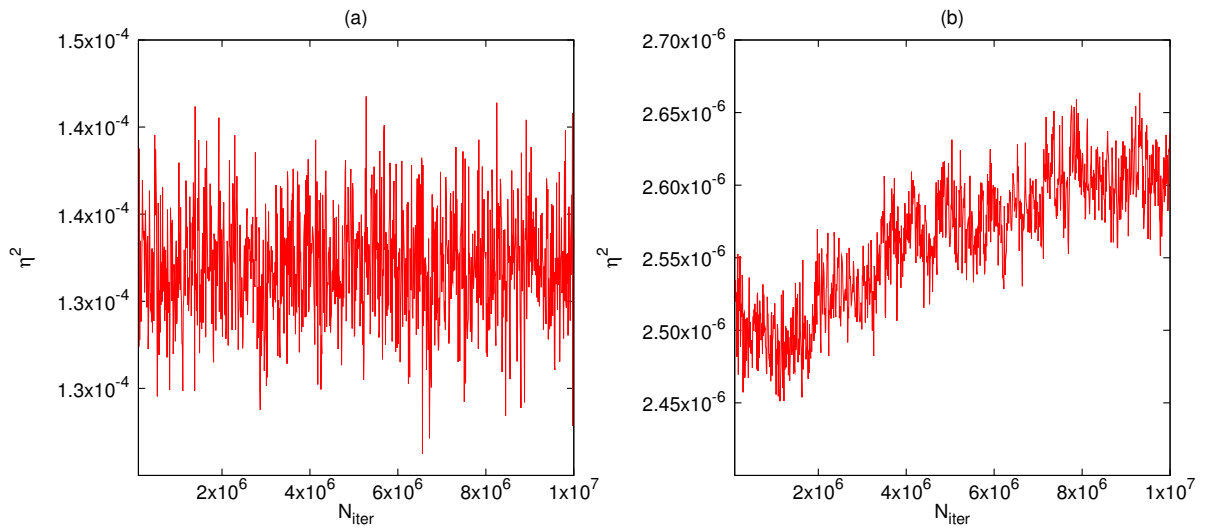

Figura 6.7: Evolución de la d.c.m. para $\gamma=0,0233$ (a) en las variables originales $\left(J_{1}, J_{2}, x_{1}, x_{2}\right)$ y $(b)$ luego de considerar solamente los puntos pertenecientes a la región $\left|x_{1}\right|+\left|x_{2}\right| \leqslant \delta$, con $\delta=0,1$.

mente, haciendo que la caracterización de la difusión no sea una tarea sencilla. Este resultado indica que, posiblemente, el tamaño del parámetro $\gamma$ sea lo suficientemente pequeño como para que el tiempo de iteración empleado no sea suficiente para revelar un crecimiento secular de $\eta^{2}$. Una forma de solucionar este inconveniente es, como hemos mencionado en la Sección 4.3, considerar un número mayor de iteraciones, por ejemplo $\mathrm{N}_{\text {iter }}=10^{8}$ tomando intervalos de tamaño $10^{5}$ iteraciones para seguir conservando la misma cantidad de puntos de salida que en los cálculos anteriores. Sin embargo, aunque sea posible reducir el tamaño de las amplitudes de las oscilaciones, el tiempo de cómputo empleado aumentaría un orden de magnitud mientras que, empleando las formas normales de orden $\mathcal{O}(7)$, el tiempo total empleado será solamente un 10 a $15 \%$ mayor que el empleado por las variables originales con $N_{i t e r}=10^{7}$. Por ende, para parámetros perturbativos muy pequeños, donde los puntos cercanos a la superficie de sección no logran reducir eficientemente las oscilaciones, necesitamos recurrir a la transformación a las formas normales. Esto es posible verlo en la Fig. 6.8, donde se ha representado la evolución de la d.c.m. mediante la aplicación de las formas normales. En este gráfico puede verse que esta técnica resulta mucho más efectiva cuando el parámetro perturbativo es muy pequeño.

A pesar de que el cálculo para $\gamma=0,0233$ utilizando las formas normales logró reducir las oscilaciones notablemente, dicha reducción no ha sido tan efectiva como en los casos para $\gamma$ mayores. La razón por la que esto ocurre podría deberse a que el parámetro $\gamma$ tenga un tamaño demasiado pequeño. Esto hace que la variación secular de las acciones que manifiesta este conjunto de condiciones iniciales sea tan lenta que su tamaño sea del orden del tamaño de las amplitu- 


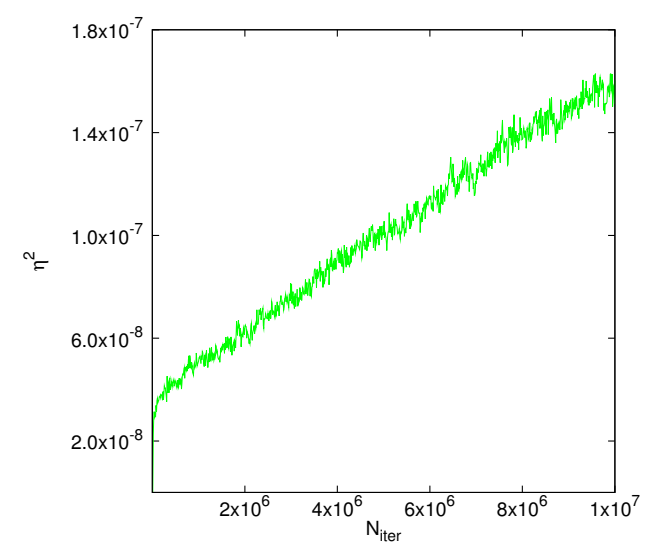

Figura 6.8: Evolución de la d.c.m. para $\gamma=0,0233$ luego de una transformación a la forma normal de orden $7\left(\Lambda_{1}, \Lambda_{2}, \theta_{1}, \theta_{2}\right)$.

des de las oscilaciones de las variables transformadas. Podemos reforzar esta hipótesis con un cálculo sencillo. El desplazamiento que experimentó la acción en la dirección de la resonancia $\left(\Lambda_{\mathrm{f}}\right)$ en toda la iteración, se puede estimar como $\eta\left(N_{i t e r}\right)$ con $N_{\text {iter }}=10^{7}$. De la Fig. 6.8 estimamos que $\eta^{2}\left(10^{7}\right) \approx 1,6 \times 10^{-7}$. Luego, el desplazamiento de la acción, $\delta \Lambda_{f}$, será $\delta \Lambda_{f} \approx 4 \times 10^{-4}$, que es un valor muy pequeño, es decir, que el conjunto de condiciones iniciales manifiesta poca difusión. Por ende vemos que, para este tamaño de valores de $\gamma$, las oscilaciones comienzan a jugar un papel más importante que en los casos de $\gamma$ mayores.

Por último, veremos qué sucede con el valor más pequeño de $\gamma$ considerado, $\gamma=0,01$. Al igual que en los paneles (a) de las Figs. 6.6 y 6.7, la evolución de la d.c.m. queda oculta por las oscilaciones en las variables originales (Fig. 6.9, panel (a)). En cuanto a las variables luego de aplicar la transformación (Fig. 6.9, panel (b)), la curva presenta oscilaciones de un tamaño mucho mayor que el manifestado por la evolución para $\gamma=0,0233$. Es evidente que, incluso luego de aplicar la transformación a la forma normal, no es posible evidenciar un crecimiento secular definido.

Una posible solución a este inconveniente podría ser considerar una iteración del mapa para tiempos más largos, por ejemplo $\mathrm{N}_{\text {iter }}=10^{k}$, con $\mathrm{k}=8,9, \ldots$. En [42] los autores llegan a considerar hasta $10^{11}$ iteraciones para poder revelar un comportamiento difusivo en un sistema ligeramente perturbado.

Para complementar este análisis, mostramos en la Fig. 6.1o la evolución de la d.c.m. considerando los puntos de la región $\left|x_{1}\right|+\left|x_{2}\right| \leqslant 0,1$. Es posible ver aquí que, si bien el valor promedio de la d.c.m. ha sido reducido en dos órdenes de magnitud $\left(\sim 10^{-4}\right.$ en la curva del panel (a) de la Fig. 6.9, y $\sim 10^{-6}$ en la curva 

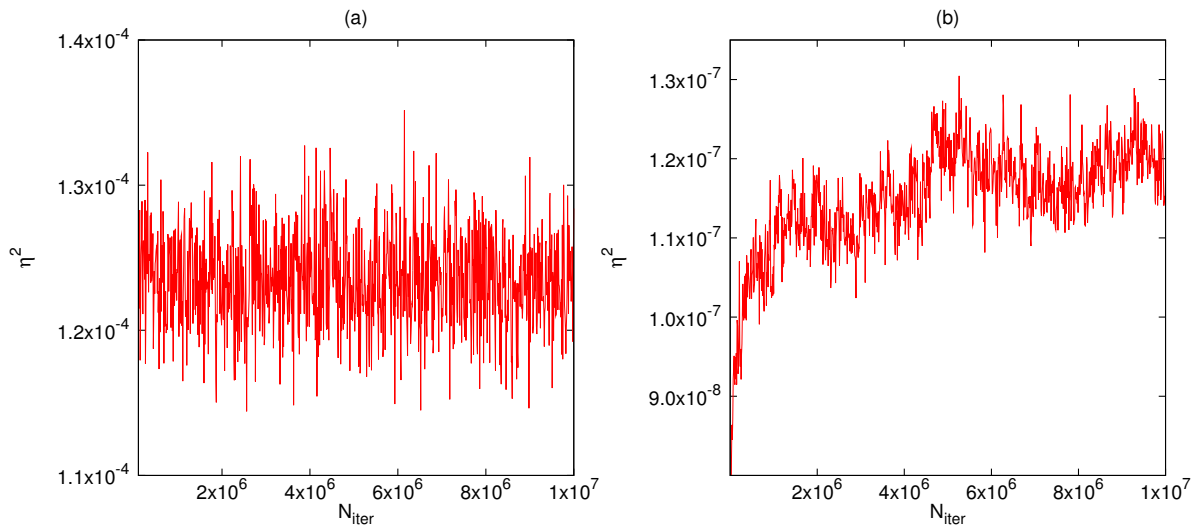

Figura 6.9: Evolución de la d.c.m. para $\gamma=0,01$ (a) en las variables originales $\left(\mathrm{J}_{1}, \mathrm{~J}_{2}, \mathrm{x}_{1}, \mathrm{x}_{2}\right)$ y (b) luego de una transformación a la forma normal de orden $7\left(\Lambda_{1}, \Lambda_{2}, \theta_{1}, \theta_{2}\right)$.

de la Fig. 6.10), esta técnica es incapaz de detectar la variación secular de la d.c.m.

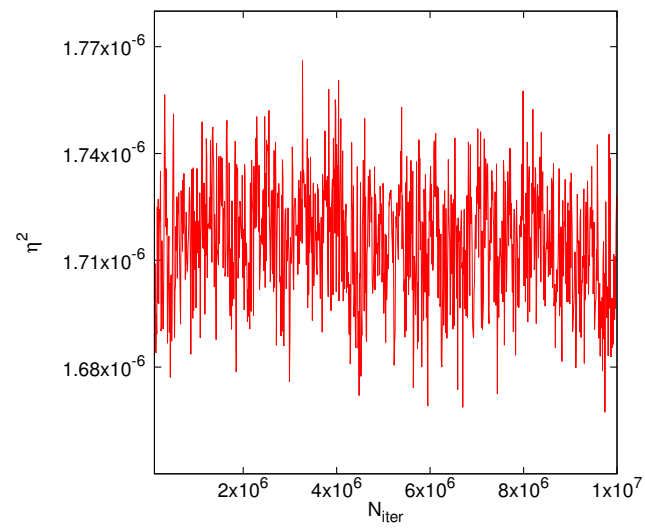

Figura 6.10: Evolución de la d.c.m. para $\gamma=0,01$ considerando solamente los puntos de la región $\left|x_{1}\right|+\left|x_{2}\right| \leqslant 0,1$.

\subsection{CARACTERIZACIÓN DE LA DIFUSIÓN EN UNA RESONANCIA SIMPLE}

Recordemos que el coeficiente de difusión $\mathcal{D}$ para un intervalo de tiempo $\Delta t$ lo suficientemente grande se define como el cociente entre la d.c.m. medida en el intervalo y la longitud de éste (Ec. (4.5)). Luego, necesitamos que la d.c.m. tenga una dependencia lineal con el tiempo. Por lo tanto, para poder realizar 
una estimación de dicho coeficiente se requiere conocer si, en un dado intervalo de tiempo, la condición inicial (o conjunto de condiciones iniciales) experimenta difusión normal.

En la Sección 4.1.I se hizo la suposición de que la evolución de la d.c.m. puede considerarse como una ley de potencias (Ec. (4.4)). Si estudiamos su comportamiento en una escala logarítmica ésta seguirá un comportamiento lineal de la forma $\log \left(\eta^{2}\right)=a+\beta \log \left(N_{\text {iter }}\right)$, donde $a=\log (\alpha), \beta \in \mathbb{R}$ son dos constantes a determinar. Por lo tanto, la caracterización de la difusión se basa en el ajuste, por el método de mínimos cuadrados, de una función lineal de este tipo, donde el valor de la pendiente $\beta$ nos va a indicar si el comportamiento es subdifusivo $(\beta<1)$, normal $(\beta=1)$ o superdifusivo $(\beta>1)$. Dado que numéricamente la probabilidad de que, luego de un ajuste, se obtenga exactamente el valor $\beta=1$ es prácticamente nula, consideraremos como régimen de difusión normal aquél donde $0,85 \leqslant \beta \leqslant 1,15$ (en [18] los autores consideraron un intervalo más amplio, $(0,75 ; 1,25)$ ). Este análisis se llevará a cabo en el conjunto de variables transformadas $\left(\Lambda_{1}, \Lambda_{2}, \theta_{1}, \theta_{2}\right)$, mediante la aplicación de las formas normales.

La Fig. 6.11 representa la evolución de la d.c.m. con respecto al número de iteraciones en el intervalo $\left(3 \times 10^{5} ; 10^{7}\right)$, siguiendo una escala logarítmica, para distintos valores de $\gamma$. Se han separado las curvas de evolución en tres paneles de acuerdo al tipo de comportamiento que manifestaron. Junto con las curvas de evolución de la d.c.m. se graficaron, con trazo negro, rectas de pendiente 1 para poder comparar los comportamientos lineales de las evoluciones y determinar en qué intervalos su crecimiento es paralelo a dichas rectas.

Para todos los $\gamma$ considerados, vamos a determinar a continuación en qué intervalos la difusión se comporta como normal. Para llevar esto a cabo, debemos estimar la pendiente $\beta$ de la evolución de la d.c.m. para los distintos valores de $\gamma$ (considerando que éstos presentan un comportamiento lineal con respecto al número de iteraciones, basándonos en la hipótesis de que la difusión se comporta como una ley de potencias en la escala lineal) y comparar los valores estimados con la unidad.

\subsubsection{Grupo $\mathscr{A}$}

Este grupo está conformado por el rango superior de valores de $\gamma(0,0545 \leqslant$ $\gamma \leqslant 0,1)$. La evolución de sus d.c.m. está representada en el panel (a) de la Fig. 6.11, mediante una escala logarítmica, en el intervalo $(4,5 ; 7)$, es decir $N_{i t e r} \in$ $\left(3 \times 10^{4} ; 10^{7}\right)$.

Para $\gamma=0,1$ y $\gamma=0,0886$ el análisis es trivial, ya que la evolución de la d.c.m. en el rango $\log \left(N_{i t e r}\right) \in(4,5 ; 7)$ es muy semejante al de una función 

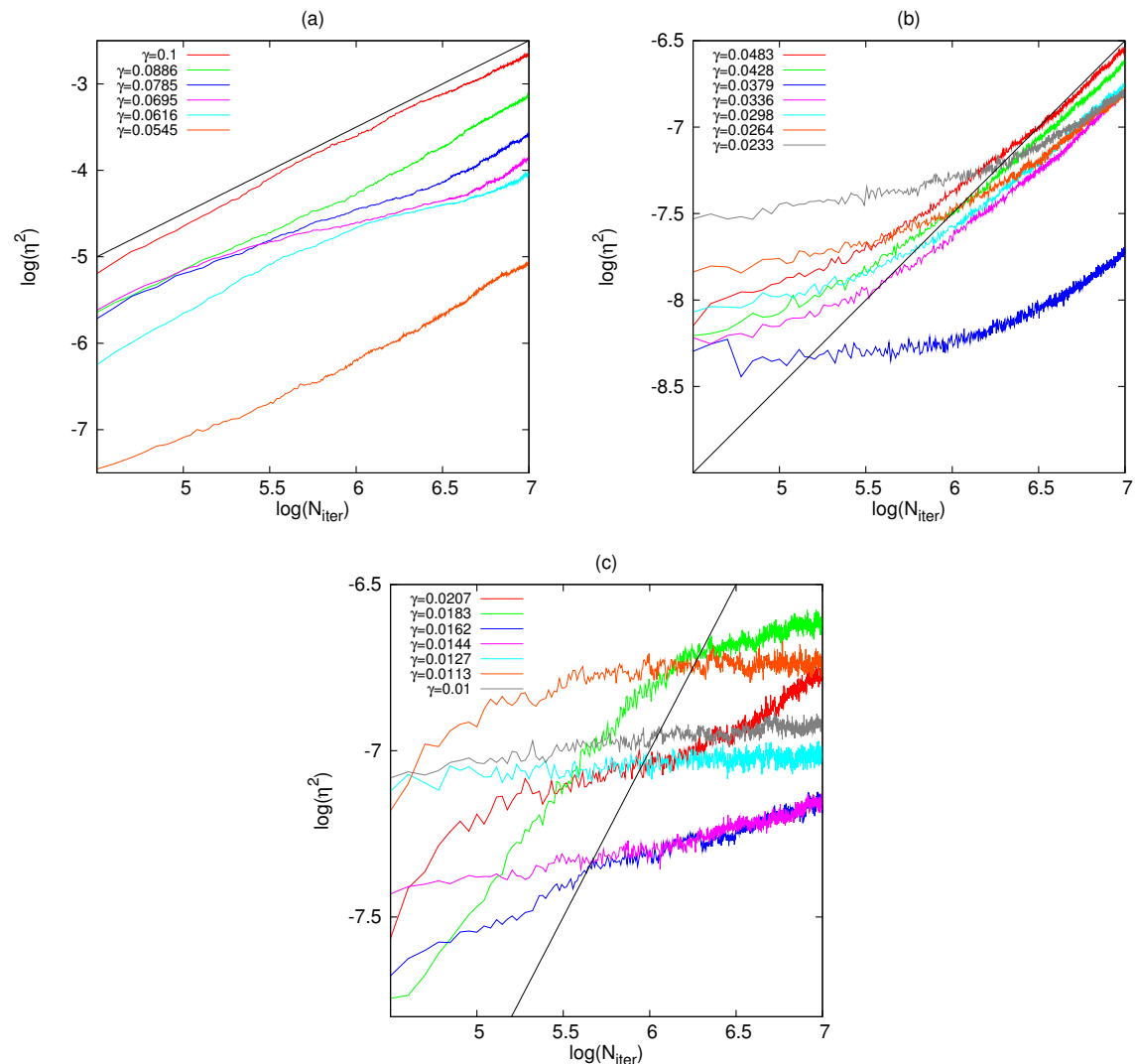

Figura 6.11: Evolución de $\log \left(\eta^{2}\right)$ con respecto a $\log \left(\mathrm{N}_{\text {iter }}\right)$ para distintos valores de $\gamma$. (a) $0,0545 \leqslant \gamma \leqslant 0,1$, llamado "grupo $\mathscr{A}^{\prime}$. (b) $0,0233 \leqslant \gamma \leqslant 0,0483$, "grupo $\mathscr{B} "$. (c) $0,01 \leqslant \gamma \leqslant 0,0207$, "grupo $\mathscr{C} "$.

lineal. Si realizamos un ajuste lineal a cada una de ellas, las rectas resultantes presentan una pendiendte muy similar a la de la recta $\log \left(\eta^{2}\right)=\log \left(\mathrm{N}_{\text {iter }}\right)$ (de aquí en más, la recta identidad). Esto indica que la difusión para $\gamma=0,1$ y $\gamma=0,0886$ puede ser considerada aproximadamente normal en ambos casos. Los resultados de la estimación de $\beta$ se resumen al final de esta sección, en la Tabla 6.1. Junto con la estimación de $\beta$ se representa también el error $E_{\beta}$ (obtenido con el método de mínimos cuadrados) con el que se realizó el ajuste.

Para los siguientes valores de $\gamma(0,0616 \leqslant \gamma \leqslant 0,0785)$ las curvas de evolución de la d.c.m. manifiestan cambios en su comportamiento con respecto al número de iteraciones. La dependencia entre $\log \left(\eta^{2}\right)$ y $\log \left(N_{\text {iter }}\right)$ para cada una de las curvas se puede dividir en tres segmentos, donde cada uno sigue un crecimiento lineal, pero con distintas pendientes. El crecimiento de la acción en el primer y tercer segmentos es cercano a la recta identidad, mientras que el segundo segmento se corresponde con una pendiente claramente menor. Esta disminución 
en la pendiente puede deberse a diversos factores. Uno de ellos, por ejemplo, podría ser que algunas de las condiciones iniciales, en un momento dado, pase muy cerca de una isla de estabilidad, quedando "pegadas"al borde exterior de ésta, fenómeno conocido en inglés como stickiness. Este efecto podría provocar una disminución en la velocidad de crecimiento de la d.c.m.

Realizando la estimación lineal de sus pendientes, considerando el intervalo de tiempo $\log \left(\mathrm{N}_{\text {iter }}\right) \in(4,5 ; 7)$, obtenemos también como resultado que la dependencia puede considerarse aproximadamente lineal para $\gamma=0,0785$, mientras que para $\gamma=0,0695$ y $\gamma=0,0616$ los valores obtenidos de la estimación son muy inferiores a la unidad, siendo $0,6 \leqslant \beta \leqslant 0,68$. La magnitud de este valor es esperable dado que, para estas dos curvas, el cambio de comportamiento fue más notorio que para $\gamma=0,0785$, repercutiendo en la estimación de $\beta$.

Si bien en la figura se puede apreciar la disminución del crecimiento de $\log \left(\eta^{2}\right)$ en $\operatorname{los}$ intervalos $\log \left(\mathrm{N}_{\text {iter }} \in(5,7 ; 6,6)\right.$ para $\gamma=0,0695$ y $\log \left(\mathrm{N}_{\text {iter }} \in\right.$ $(6 ; 6,7)$ para $\gamma=0,0616$ vemos que, luego de estos intervalos, las curvas vuelven a manifestar un crecimiento. Este efecto podría ocurrir debido a que, si la disminución se produjo por un efecto tipo sticky, estas condiciones iniciales lograron "despegarse"de la isla de estabilidad alrededor de la cual se movían, logrando nuevamente un incremento en la d.c.m. Por lo tanto, si consideramos un tiempo de integración mayor, sería posible observar una tendencia aproximadamente lineal en la curva. Realizando este cálculo podemos apreciar en la Fig. 6.12 que, para $\log \left(\mathrm{N}_{\text {iter }}\right) \gtrsim 6,5$ la evolución de la d.c.m. para ambas curvas es aproximadamente lineal. Más aún, si realizamos ahora la estimación de $\beta$ para el intervalo $\log \left(\mathrm{N}_{\text {iter }}\right) \in(5,5 ; 8)$ obtenemos $\beta \approx 1,047$ para $\gamma=0,0616 \mathrm{y}$ $\beta \approx 1,099$ para $\gamma=0,0695$.

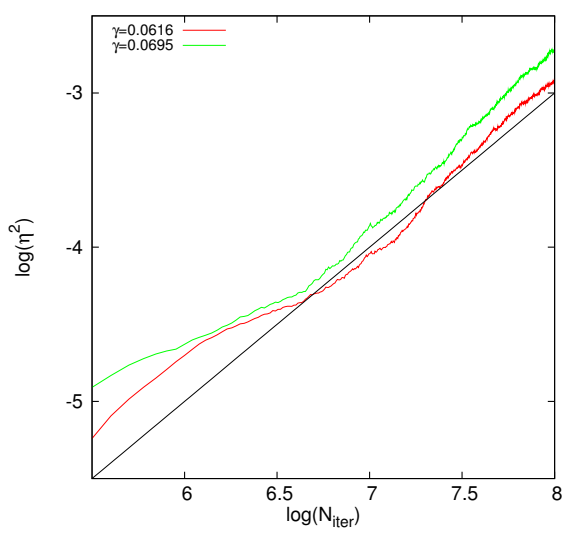

Figura 6.12: Evolución de $\log \left(\eta^{2}\right)$ con respecto a $\log \left(\mathrm{N}_{\text {iter }}\right)$ para $\gamma=0,0616$ y $\gamma=$ 0,0695 , para $N_{i t e r}=10^{8}$. 
Por último, para $\gamma=0,0545$, vemos que la dependencia entre $\log \left(\eta^{2}\right)$ y $\log \left(N_{\text {iter }}\right)$ se aparta de la linealidad. Sin embargo, se puede apreciar que dicho apartamiento no es significativo. Si aproximamos esta curva como dos rectas, una en el intervalo $\log \left(N_{\text {iter }}\right) \in(4,5 ; 6)$ y la otra en $\log \left(N_{\text {iter }}\right) \in(6 ; 7)$, las estimaciones de sus pendientes serán $\beta \approx 0,874$ y $\beta \approx 1,184$, mientras que al aproximar a la curva con una única recta, la estimación de la pendiente obtenida es $\beta \approx 1,097$, todos valores cercanos a la unidad. Por ende, es posible aproximar esta dependencia con una recta en el intervalo $(4,5 ; 7)$, cuya estimación de su pendiente permite suponer una difusión aproximadamente normal.

\begin{tabular}{|c|c|c|}
\hline$\gamma$ & $\beta$ & $E_{\beta}(\%)$ \\
\hline \hline 0,1 & 0,920 & 0,1359 \\
0,0886 & 1,115 & 0,1733 \\
0,0785 & 0,858 & 0,5066 \\
0,0695 & 1,099 & 0,3773 \\
0,0616 & 1,047 & 0,4329 \\
0,0545 & 1,143 & 0,2262 \\
\hline
\end{tabular}

Tabla 6.1: Estimación de la pendiente del ajuste de la recta $\log \left(\eta^{2}\right)=a+\beta \log \left(N_{\text {iter }}\right)$ para distintos valores de $\gamma$ en el rango $0,0545 \leqslant \gamma \leqslant 0,1$, con $N_{\text {iter }} \in$ $\left(3 \times 10^{4} ; 10^{7}\right)$ para $\gamma=0,1 ; 0,0886 ; 0,0785$ y $N_{i t e r} \in\left(3 \times 10^{5} ; 10^{8}\right)$ para $\gamma=0,0695 ; 0,616$. La primera columna muestra el valor del parámetro perturbativo $\gamma$, mientras que las dos últimas columnas exhiben el valor de la pendiente $\beta$ del ajuste y el error porcentual $\left(E_{\beta}\right)$ con el que fue estimada.

\subsubsection{Grupo $\mathscr{B}$}

Este segundo grupo corresponde al rango de valores $0,0233 \leqslant \gamma \leqslant 0,0483$, cuyas curvas de evolución de la d.c.m. se encuentran representadas en la Fig. 6.11, panel (b). En este gráfico es posible apreciar que todas estas curvas presentan una tendencia similar, comenzando con un crecimiento lento, con una velocidad inferior a la correspondiente para difusión normal, y va aumentando hasta alcanzar valores cercanos a 1 para valores como $\gamma=0,0483$ y $\gamma=0,0428$. Para el resto de los valores de $\gamma$ el incremento no es suficiente para lograr una dependencia lineal cuya pendiente sea cercana a la unidad.

Claramente se ve que estas curvas no manifiestan un comportamiento lineal, por lo que ninguna de estas evoluciones puede considerarse que se encuentren en un régimen de difusión normal.

Este comportamiento podría ocurrir debido a que, al disminuir el tamaño de la perturbación (disminuyendo el valor de $\gamma$ ), el tamaño de la resonancia 
comienza a ser comparable con el de las resonancias que la cruzan, teniendo éstas una mayor influencia en la dinámica. En la Fig. 6.13 se muestra un mapa del valor del FLI cerca del punto $y_{1}^{*}=y_{2}^{*}=\sqrt{3}+0,08$, comprendida en la región $\left(y_{1}, y_{2}\right) \in[1,806 ; 1,818) \times[1,806 ; 1,818)$.
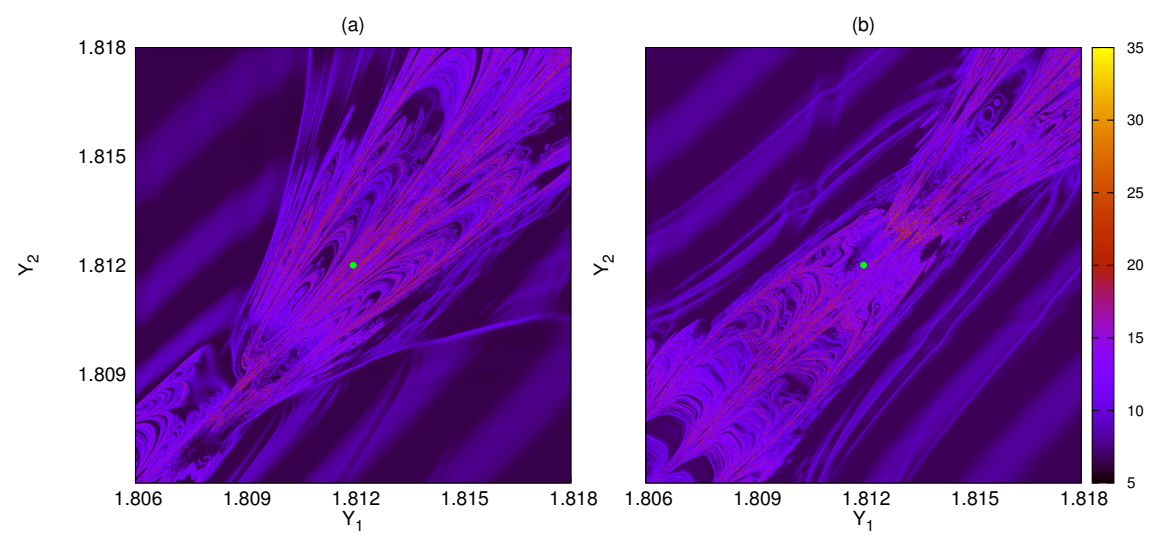

Figura 6.13: Valores del FLI para una grilla de condiciones iniciales en la región $[1,806 ; 1,818) \times[1,806 ; 1,818)$ para $\gamma=0,0233(a), y \gamma=0,0428(b)$.

Es posible ver aquí que los valores de $\ln (\mathrm{FLI})$ en la capa estocástica de la resonancia son mucho menores que los correspondientes a las mismas condiciones iniciales en el caso $\gamma=0,1$ (Fig. $5 \cdot 3$ (a)). Más aún, algunos de estos valores están próximos al régimen de regularidad, produciendo una alteración a la estructura dinámica del sistema. Esta alteración podría ser el causante de que la velocidad de la difusión en este tipo de escenarios sea inferior a la correspondiente a la forma normal. También es posible apreciar que en el gráfico aparecen ciertas bandas de color más claro, asociadas a las resonancias de alto orden que en casos con un mayor grado de caos no se podían apreciar.

Por otro lado, el ancho de la capa estocástica de la resonancia se vuelve más angosto que en el caso $\gamma=0,1$. Tanto este efecto como el anterior, podrían hacer que el desplazamiento de la acción en la dirección de la resonancia ocurra de una manera más lenta. Por ende, si consideramos como correcta esta hipótesis, una posible solución sería considerar un número de iteraciones mayor, digamos $\mathrm{N}_{\text {iter }}=10^{8}$.

En la Fig. 6.14 se encuentra representada la evolución de la d.c.m. para los mismos valores de $\gamma$, para $\mathrm{N}_{\text {iter }}=10^{8}$.

$\mathrm{Al}$ considerar un número mayor de iteraciones, es posible ahora ver una dependencia más próxima a la lineal. Más aún, realizando la estimación de la pendiente $\beta$, obtenemos valores cercanos a 1, evidenciando un comportamiento aproximadamente normal. En la Tabla 6.2 se representan las estimaciones realizadas. 


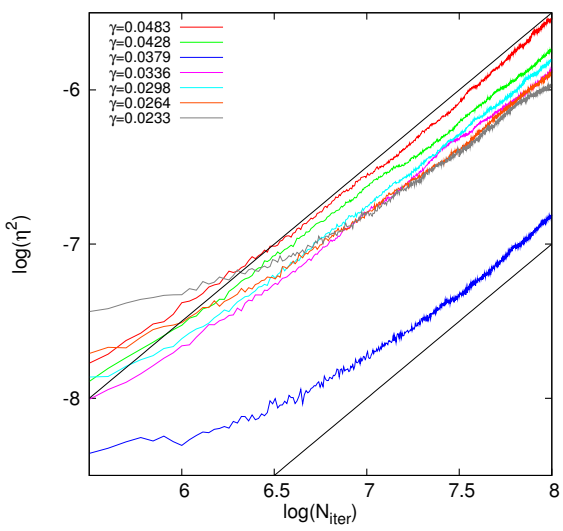

Figura 6.14: Evolución de $\log \left(\eta^{2}\right)$ con respecto a $\log \left(\mathrm{N}_{\text {iter }}\right)$ para el rango de valores $0,0233 \leqslant \gamma \leqslant 0,0483$, en el intervalo $\log \left(N_{\text {iter }}\right) \in(5,5 ; 8)$.

\begin{tabular}{|c|c|c|}
\hline$\gamma$ & $\beta$ & $E_{\beta}(\%)$ \\
\hline \hline 0,0483 & 0,998 & 0,1916 \\
0,0428 & 0,895 & 0,1151 \\
0,0379 & 0,851 & 0,3917 \\
0,0336 & 0,915 & 0,1282 \\
0,0298 & 0,933 & 0,0923 \\
0,0264 & 0,884 & 0,2286 \\
0,0233 & 0,807 & 0,3398 \\
\hline
\end{tabular}

Tabla 6.2: Estimación de la pendiente del ajuste de la recta $\log \left(\eta^{2}\right)=a+\beta \log \left(N_{i t e r}\right)$ para distintos valores de $\gamma$ en el rango $0,0233 \leqslant \gamma \leqslant 0,0545$, con $\log \left(\mathrm{N}_{\text {iter }}\right) \in$ $(5,5 ; 8)$. La primera columna muestra el valor del parámetro perturbativo $\gamma$, mientras que las dos últimas columnas exhiben el valor de la pendiente $\beta$ del ajuste y el error porcentual $\left(E_{\beta}\right)$ con el que fue estimada.

\subsubsection{Grupo $\mathscr{C}$}

Este último conjunto de parámetros corresponde al rango de valores $0,01 \leqslant$ $\gamma \leqslant 0,0207$, y se encuentra representado en el panel (c) de la Fig. 6.11. Las curvas mostradas en esta figura revelan que, para este número de iteraciones, la velocidad de incremento de $\log \left(\eta^{2}\right)$ es claramente inferior a la de la recta unidad. Este comportamiento es consistente con el análisis llevado a cabo hasta aquí dado que, al considerar valores de $\gamma$ menores a los empleados en 
la Sección 6.3.2, el tamaño de la perturbación disminuye, y los efectos de las resonancias de alto orden se vuelven más significativas.

Continuando con la hipótesis establecida en la Sección 6.3.2 para el grupo $\mathscr{B}$, la velocidad a la que aumenta secularmente $\eta^{2}$ debería incrementarse hasta alcanzar un valor cercano a 1 , si se considera un número de iteraciones suficientemente grande. Si realizamos el cálculo de $\log \left(\eta^{2}\right)$ para $N_{i t e r} \in\left(0 ; 10^{8}\right)$ (ver Fig. 6.15) observamos que, si bien algunas de las curvas (las correspondientes a $0,0162 \leqslant \gamma \leqslant 0,0207)$ aceleran su incremento, el nuevo valor final de $\mathrm{N}_{\text {iter }}$ no es suficiente como para que se manifieste un régimen de difusión cercana a la normal.

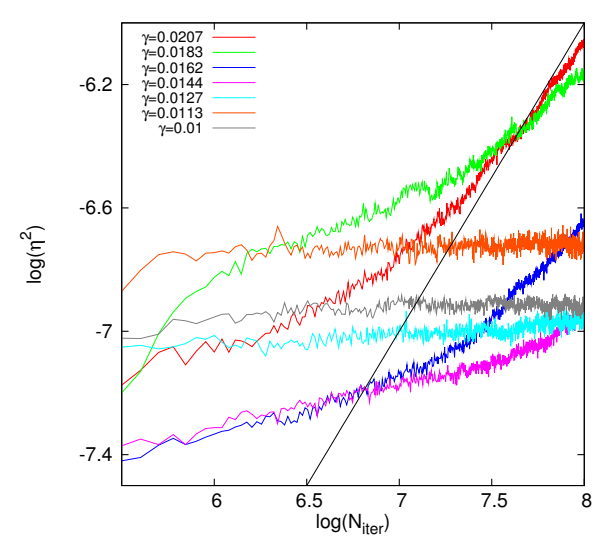

Figura 6.15: Evolución de $\log \left(\eta^{2}\right)$ con respecto a $\log \left(\mathrm{N}_{\text {iter }}\right)$ para el rango de valores $0,01 \leqslant \gamma \leqslant 0,0207$, en el intervalo $\log \left(N_{i t e r}\right) \in(5,5 ; 8)$.

En cambio, si observamos el segundo conjunto de valores más pequeño $(0,01 \leqslant \gamma \leqslant 0,0144)$, vemos que éstos presentan un incremento casi nulo. Por ende, podemos concluir que para el grupo $\mathscr{C}$ el comportamiento de la difusión va a ser claramente no normal, por lo que no profundizaremos en su estudio.

A diferencia de lo ocurrido en la Sección 6.3.2, considerar $N_{i t e r}=10^{8}$ no fue suficiente para que las curvas analizadas en el grupo $\mathscr{C}$ experimenten difusión normal. Por consiguiente, quizá sea necesario adoptar un número de iteraciones aún mayor (digamos, $10^{9}$ ) como para que las curvas pertenecientes al grupo $\mathscr{C}$ experimenten un régimen de difusión normal.

Como una manera de comprobar empíricamente que nuestra hipótesis podría ser correcta, en la Fig. 6.16 se muestra la evolución de la d.c.m. para $\gamma=0,0207$ y $\gamma=0,0144$, considerando esta vez $\mathrm{N}_{\text {iter }}=10^{9}$.

Es posible notar que, para este número de iteraciones, la evolución de la d.c.m. correspondiente a $\gamma=0,0207$ presenta una variación de las acciones similar a la difusión normal. Sin embargo, para $\gamma=0,0144$, nuevamente nos 


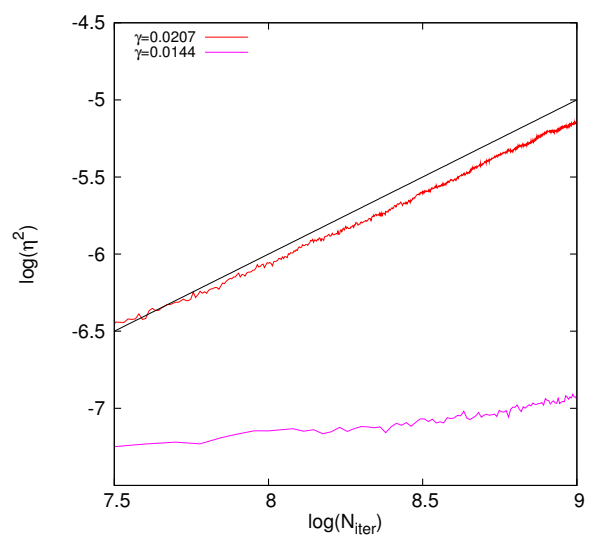

Figura 6.16: Evolución de $\log \left(\eta^{2}\right)$ con respecto a $\log \left(\mathrm{N}_{\text {iter }}\right)$ para $\gamma=0,0207$ y $\gamma=$ 0,0144 , en el intervalo $\log \left(N_{\text {iter }}\right) \in(6,5 ; 9)$.

encontramos en una situación en la que todavía no logró ingresar en dicho régimen, necesitando quizá aumentar aún más el número de iteraciones. En este trabajo no nos vamos a abocar al estudio de la variación de las acciones para un valor de $\mathrm{N}_{\text {iter }}$ tan elevado dado que, como ha sido explicado en la Sección 4.2.1, el resultado obtenido para un número tan grande de iteraciones carece de sentido físico. Este resultado tuvo como objetivo simplemente comprobar que, si se incrementa lo suficiente el número de iteraciones, sería posible lograr que la evolución de la d.c.m. presente un carácter similar a la difusión normal, teniendo en cuenta que no logró manifestarlo para un número menor de iteraciones.

\subsubsection{Estimación del coeficiente de difusión $\mathcal{D}$}

Siguiendo con el análisis de la sección anterior, una vez caracterizada la difusión aproximadamente como normal, podemos estimar con qué velocidad se manifiesta esta variación de $\eta^{2}$, esto es, el coeficiente de difusión $\mathcal{D}$. Este estudio será llevado a cabo para aquellos valores de $\gamma$ en los cuales hemos detectado difusión normal $(0,0233 \leqslant \gamma \leqslant 0,1)$. En esta sección simplemente mostraremos el análisis de algunos de estos valores.

Dado que, en los intervalos escogidos, la d.c.m. experimentó difusión aproximadamente normal (es decir, $\beta \approx 1$ ), existirá una relación lineal entre $\eta^{2} \mathrm{y}$ $\mathrm{N}_{\text {iter }}$ de la forma $\eta^{2}=\mathcal{D} \mathrm{N}_{\text {iter }}+v$, donde $v$ es un parámetro a determinar ([18], Ec. (52)). En esta expresión, $\mathcal{D}$ es el coeficiente de difusión que se va a estimar, y nos proporcionará información sobre la velocidad a la que está variando la d.c.m. del conjunto de condiciones iniciales, a lo largo de la resonancia $1: 1$, en las vecindades del punto $y_{1}^{*}=y_{2}^{*}=\sqrt{3}+\delta$, con $\delta=0,08$. La estimación de 
$\mathcal{D}$, como así también la determinación del error $\mathrm{E}_{\mathcal{D}}$, serán realizadas mediante un ajuste de mínimos cuadrados.

Como se ha mostrado en la Fig 6.3 para $\gamma=0,1$, la amplitud de las oscilaciones es muy similar en ambos conjuntos de variables, por lo que se puede estimar los dos valores de $\mathcal{D}$, obteniendo $\mathcal{D}=1,939 \times 10^{-10}$ para las variables originales y $\mathcal{D}=2,131 \times 10^{-10}$ para las nuevas variables. Efectivamente, los valores estimados son muy similares, difiriendo solamente en un $10 \%$ aproximadamente. Adoptaremos como valor de $\mathcal{D}$ el correspondiente al nuevo conjunto de variables.

Para asegurarnos de que el resultado obtenido corresponde a la d.c.m. en la dirección de la resonancia, hemos calculado la superficie de sección $\sqrt{x_{1}^{2}+x_{2}^{2}} \leqslant$ $10^{-3}$, que se muestra en el panel (a) de la Fig. 6.17 mediante puntos verdes, superpuesto a un mapa de valores del FLI.
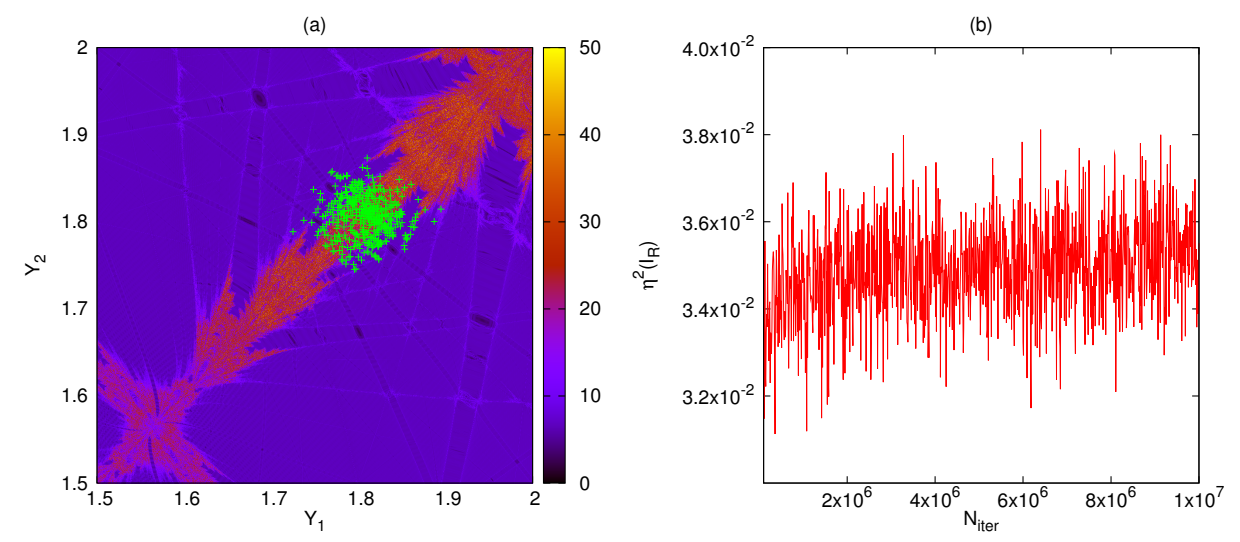

Figura 6.17: (a) Superficie de sección $\sqrt{x_{1}^{2}+x_{2}^{2}} \leqslant 10^{-3}$ para $\gamma=0,1$ y $N_{\text {iter }}=10^{7}$ iteraciones. (b) Evolución temporal de $\eta^{2}$ de la acción $I_{R}$.

Podemos observar en la figura que algunas órbitas se mueven fuera de la resonancia $1: 1$, por regiones donde se encuentran resonancias de mayor orden. Sin embargo, se puede ver en la figura que la fracción de puntos verdes que se ubican sobre dichas resonancias es mucho menor que el número de puntos sobre la resonancia $1: 1$. Con esto hemos concluído que el movimiento sobre la resonancia $1: 1$ es predominante. A esto se suma que, debido a que se está considerando una región cercana a la superficie de sección, pero de un espesor finito, es posible que algunas de estas condiciones iniciales se encuentre efectivamente sobre la capa estocástica de la resonancia principal, pero fuera de la sección. Por lo tanto, al haber escogido un espesor no nulo, al realizar la proyección de los puntos de esta región sobre el mapa del FLI, en la figura se verán como si estuvieran fuera de la resonancia. En consecuencia, podríamos 
considerar que, globalmente, el conjunto se mueve sobre la resonancia principal.

Para reforzar esta afirmación realizaremos lo siguiente. En la Sección 4.2.1 hemos definido $I_{R}$ como la acción en la dirección perpendicular a la resonancia. A partir de la Ec. (4.22), al igual que se hizo para $I_{f}$ en las variables originales podemos obtener la expresión para $I_{R}$ en las nuevas variables, que será $I_{R}=\Lambda_{1}-\Lambda_{2}$. Luego, la evolución de $\eta^{2}\left(I_{R}\right)$ mide la difusión en esta dirección. Como la capa estocástica debería tener un ancho finito, si el conjunto de condiciones iniciales permanece en ella, el valor de $\eta^{2}\left(I_{R}\right)$ tendría un valor límite superior, que coincidiría con el ancho de la capa estocástica. En el panel (b) de la Fig. 6.17 se presenta un gráfico de la evolución de la $\eta^{2}$ en la dirección de $I_{R}$ con respecto al número de iteraciones. De esta figura podemos determinar que esta magnitud crece lentamente, tendiendo a un valor aproximadamente constante. Su valor promedio es aproximadamente $3,5 \times 10^{-2}$, valor que se asemeja al tamaño de la capa estocástica observado en el panel (b) de la Fig. 6.17.

Del mismo modo que se ha hecho para $\gamma=0,1$, podemos estimar el valor de $\mathcal{D}$ para $\gamma=0,0785$ en ambos conjuntos de variables. Haciendo esto obtenemos $\mathcal{D}=2,33 \times 10^{-11}$ para el conjunto original de variables y $\mathcal{D}=2,485 \times 10^{-11}$ en el nuevo conjunto. Adoptaremos nuevamente el valor correspondiente al nuevo conjunto de variables, debido a que en éste la amplitud de las oscilaciones es ligeramente menor.

La superficie de sección $\sqrt{x_{1}^{2}+x_{2}^{2}} \leqslant 10^{-3}$ de la evolución de $\eta^{2}$ para $\gamma=$ 0,0785 (Fig. 6.18, panel (a)) muestra que los valores iniciales del conjunto están todos confinados a la resonancia principal, a excepción de un conjunto pequeño de éstos que se han dispersado por otras resonancias de mayor orden, presumiblemente por las mismas razones detalladas para $\gamma=0,1$.

El panel (b) de la Fig. 6.18 representa la evolución de $\eta^{2}\left(I_{R}\right)$. Su comportamiento nos permite concluir que, si bien la curva presenta un ligero crecimiento (al igual que para $\gamma=0,1$ ), para $N_{i t e r} \geqslant 5 \times 10^{6}$ ésta se estabiliza a un comportamiento constante, con un valor cercano a $\sim 2,5 \times 10^{-2}$ y que es similar al ancho que se observa en el panel (a) de la Fig. 6.18.

Con respecto a $\gamma=0,0616$, en la Sección 6.3.1 encontramos que, en el intervalo $\mathrm{N}_{\text {iter }} \in\left(0 ; 10^{7}\right)$, la evolución de la d.c.m. no correspondía a un régimen de difusión normal, por lo que carece de sentido la estimación de un coeficiente de difusión para ese intervalo. Sin embargo, dado que para $N_{\text {iter }} \in\left(0 ; 10^{8}\right)$ la evolución sí se manifestó como aproximadamente normal, estimamos $\mathcal{D}$ en este intervalo, obteniendo como resultado $\mathcal{D}=1,275 \times 10^{-11}$. Se ha encontrado que, para la estimación de $\mathcal{D}$ en el caso $\gamma=0,0695$, debemos utilizar el mismo intervalo que para $\gamma=0,0616$.

En el panel (a) de la Fig. 6.19 se puede ver la superficie de sección, donde claramente la evolución de las condiciones iniciales se encuentran sobre la re- 

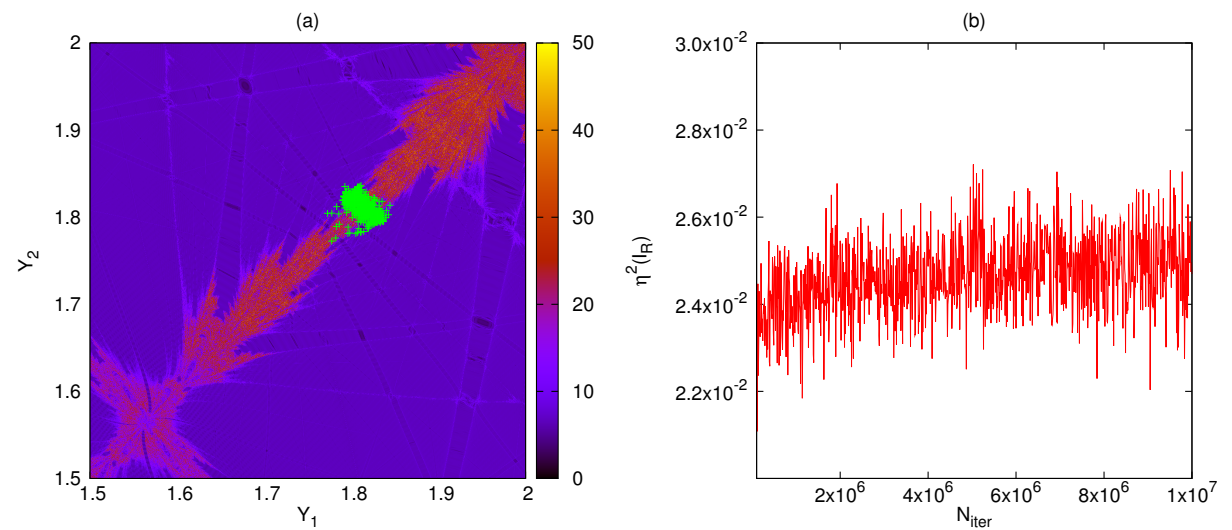

Figura 6.18: (a) Superficie de sección $\sqrt{x_{1}^{2}+x_{2}^{2}} \leqslant 10^{-3}$ para $\gamma=0,0785$ y $\mathrm{N}_{\text {iter }}=10^{7}$ iteraciones. (b) Evolución temporal de $\eta^{2}$ de la acción $I_{R}$.

sonancia $1: 1$, quedando solamente algunos pocos puntos fuera de ésta. Este resultado se complementa con la cuantificación de la d.c.m. en la dirección de $I_{R}$ (panel (b)) donde, si bien el crecimiento de esta cantidad es del orden de $10 \%$, hay que tener en cuenta que, para el número de iteraciones considerado, la velocidad con que aumenta $\eta^{2}\left(I_{R}\right)$ es suficientemente lenta como para establecer que el valor de $\eta^{2}\left(I_{R}\right)$ es aproximadamente constante.
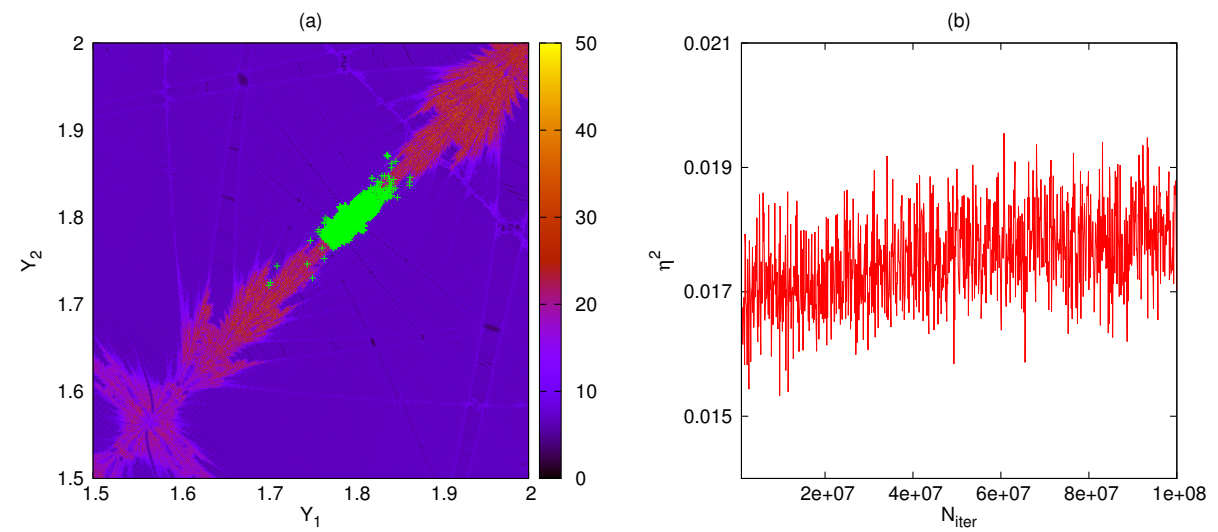

Figura 6.19: (a) Superficie de sección $\sqrt{x_{1}^{2}+x_{2}^{2}} \leqslant 10^{-3}$ para $\gamma=0,0616$, con $\mathrm{N}_{\text {iter }}=$ $10^{8}$. (b) Evolución temporal de $\eta^{2}$ de la acción $I_{R}$.

Para la evolución correspondiente a $\gamma=0,0545$, la Tabla 6.I muestra que el valor de $\beta$ es cercano a 1, por lo que la difusión es aproximadamente normal. La estimación de $\mathcal{D}$ en el nuevo conjunto de variables es $\mathcal{D}=8,865 \times 10^{-13}$. 
La Fig. 6.20, en el panel (a), muestra la superficie de sección para este valor de $\gamma$. Se puede ver que la superficie de sección de las órbitas permanece confinada a una región cercana a la resonancia. Sin embargo, hay un número de puntos en la gráfica que se encuentran fuera de la sección de la resonancia. Teniendo en cuenta que la estimación de $\beta$ que figura en la Tabla 6.1 muestra que el comportamiento de la d.c.m. es aproximadamente normal, estos puntos podrían corresponder simplemente al hecho de estar considerando una superficie de sección de espesor no nulo, como se sugirió para $\gamma=0,1$.
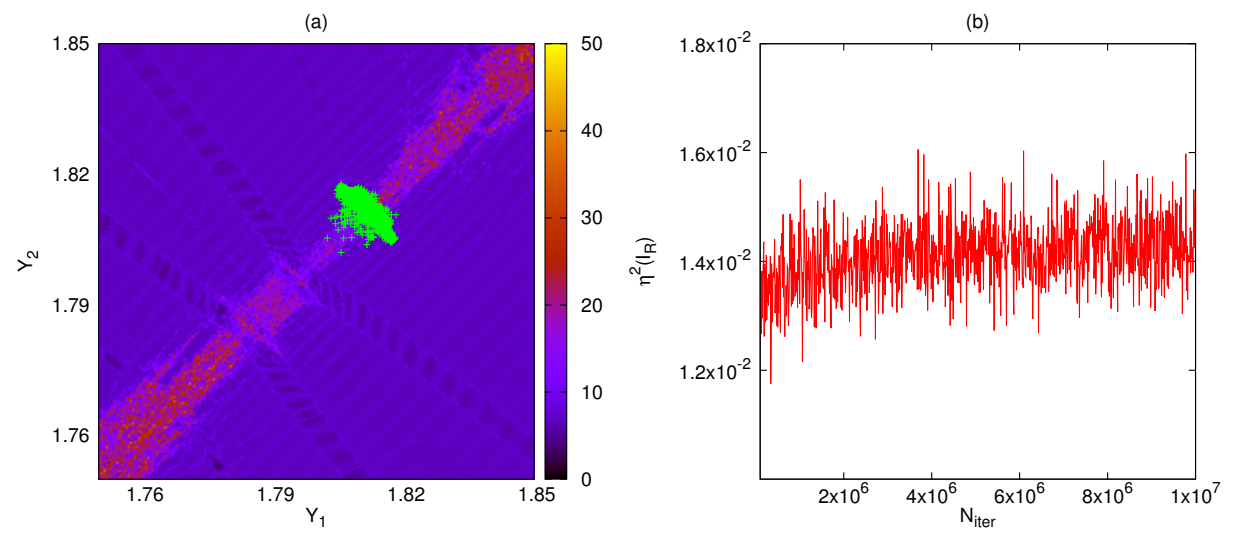

Figura 6.20: (a) Superficie de sección $\sqrt{x_{1}^{2}+x_{2}^{2}} \leqslant 10^{-3}$ para $\gamma=0,0545$, con $\mathrm{N}_{\text {iter }}=$ $10^{7}$. (b) Evolución temporal de $\eta^{2}$ de la acción $I_{R}$.

Con respecto a la evolución de la d.c.m. correspondiente al rango de valores de $\gamma$ del grupo $\mathscr{B}$ analizaremos, como representantes de dicho grupo, los casos de $\gamma=0,0483$ y $\gamma=0,0264$, dado que las curvas de evolución de $\log \left(\eta^{2}\right)$ de todas ellas presentaron un comportamiento muy similar. Recordemos también que se obtuvieron estimaciones de $\beta$ correspondientes a difusión muy cercana a la normal, por lo que es posible estimar un valor de $\mathcal{D}$ para $N_{\text {iter }} \in\left(0 ; 10^{8}\right)$.

Para $\gamma=0,0483$, el coeficiente de difusión estimado es $\mathcal{D}=2,934 \times 10^{-14}$, mientras que para $\gamma=0,0264$ la estimación obtenida es $\mathcal{D}=1,278 \times 10^{-14}$. En el panel (a) de la Fig. 6.21 se muestra la superficie de sección para $\gamma=$ 0,0483 . Vemos que la mayoría de los puntos correspondientes a la evolución del conjunto de condiciones iniciales se desplaza sobre la resonancia $1: 1$. Sin embargo, existe un pequeño grupo de éstos que, visiblemente, se encuentran fuera de dicha resonancia. Estos últimos puntos se encuentran en una región donde distintas resonancias cruzan a la $1: 1$ (la región cercana a $(1,808,1,808)$ ) y, en consecuencia, aumenta el ancho de la capa estocástica. Luego, debido a que, como el valor de $\eta^{2}\left(I_{R}\right)$ es aproximadamente constante (Fig. 6.21, panel 
(b)), es posible que este conjunto de puntos se encuentre sobre la región de la capa que está fuera de la sección, es decir, donde $\sqrt{x_{1}^{2}+x_{2}^{2}}>10^{-3}$.
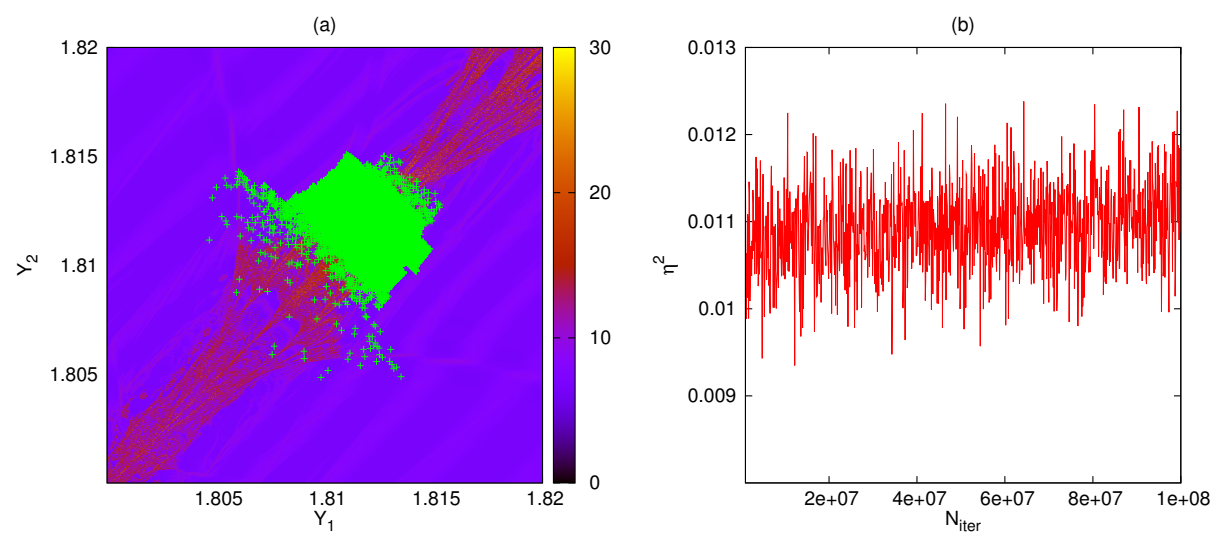

Figura 6.21: (a) Superficie de sección $\sqrt{x_{1}^{2}+x_{2}^{2}} \leqslant 10^{-3}$ para $\gamma=0,0483$, con $N_{i t e r}=$ $10^{8}$. (b) Evolución temporal de $\eta^{2}$ de la acción $I_{R}$.

En cuanto a $\gamma=0,0264$, su superficie de sección, que se encuentra representada en el panel (a) de la Fig. 6.22, presenta un desplazamiento más acotado, permaneciendo los puntos dentro de la resonancia (si bien se ven muchos puntos cerca de la resonancia pero fuera de ella, es importante destacar que las escalas para $Y_{1}$ e $Y_{2}$ en esta gráfica son mucho más pequeñas que en los gráficos anteriores). Esta conclusión es apoyada por el panel (b) de la Fig. 6.22, donde se muestra que los valores de $\eta^{2}\left(I_{R}\right)$ son aproximadamente constantes. Estos últimos resultados apoyan la hipótesis de que la difusión ocurre en la dirección de la resonancia.

El resultado de las estimaciones de $\mathcal{D}$ para todos los valores de $\gamma$ en el rango $0,0233 \leqslant \gamma \leqslant 0,1$ se encuentran resumidos en la Tabla 6.3, donde se muestra, junto con los coeficientes estimados, el error $\left(E_{\mathcal{D}}\right)$ con que se han determinado.

\subsection{CARACTERIZACIÓN DE LA DIFUSIÓN EN LAS CERCANÍAS DE UN CRU- CE DE RESONANCIAS}

En la Sección 6.3 se presentaron los resultados del cálculo de la evolución de la d.c.m. a lo largo de la resonancia $1: 1$ en un punto lo suficientemente alejado de un cruce de resonancias, haciendo válido el uso del desarrollo de las formas normales para una resonancia simple (ver Sección 5.2.2.2). A continuación veremos cómo afecta a estos resultados la consideración de un punto más cercano a un cruce. El punto escogido corresponde a considerar $y_{1}^{*}=y_{2}^{*}=\sqrt{3}+\delta$, con 

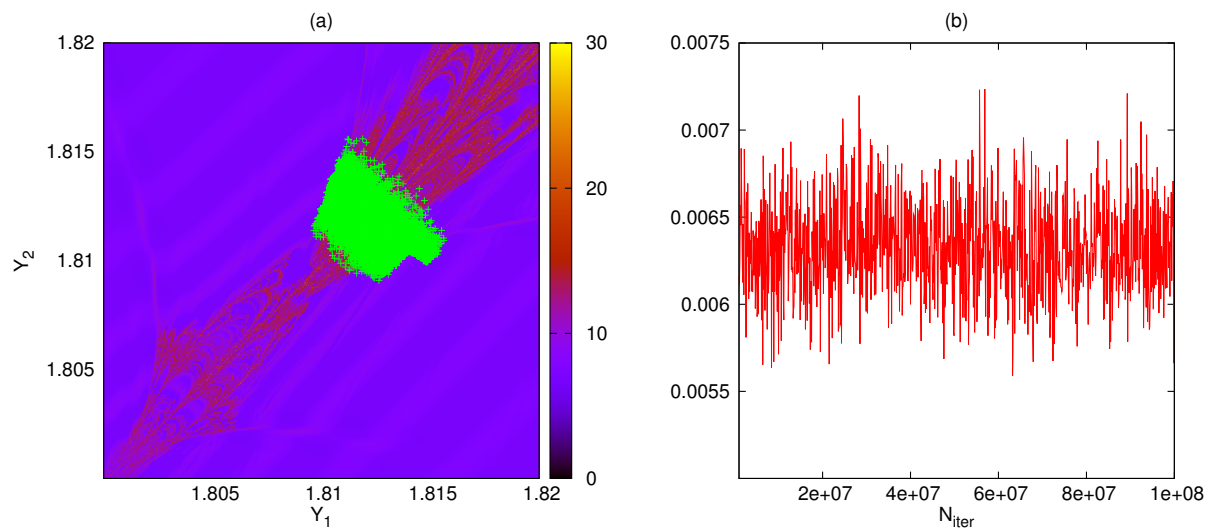

Figura 6.22: (a) Superficie de sección $\sqrt{x_{1}^{2}+x_{2}^{2}} \leqslant 10^{-3}$ para $\gamma=0,0264$, con $\mathrm{N}_{\text {iter }}=$ $10^{8}$. (b) Evolución temporal de $\eta^{2}$ de la acción $I_{R}$.

\begin{tabular}{|c|c|c|}
\hline$\gamma$ & $\mathcal{D}$ & $\mathrm{E}_{\mathcal{D}}(\%)$ \\
\hline \hline 0,1 & $2,131 \times 10^{-10}$ & 0,1356 \\
0,0886 & $7,440 \times 10^{-11}$ & 0,1741 \\
0,0785 & $2,485 \times 10^{-11}$ & 0,3047 \\
0,0695 & $1,994 \times 10^{-11}$ & 0,1988 \\
0,0616 & $1,275 \times 10^{-11}$ & 0,1954 \\
0,0545 & $8,865 \times 10^{-13}$ & 0,2699 \\
\hline
\end{tabular}

\begin{tabular}{|c|c|c|}
\hline$\gamma$ & $\mathcal{D}$ & $\mathrm{E}_{\mathcal{D}}(\%)$ \\
\hline \hline 0,0483 & $2,934 \times 10^{-14}$ & 0,1578 \\
0,0428 & $1,765 \times 10^{-14}$ & 0,1199 \\
0,0379 & $1,488 \times 10^{-15}$ & 0,1834 \\
0,0336 & $1,281 \times 10^{-14}$ & 0,1859 \\
0,0298 & $1,540 \times 10^{-14}$ & 0,1079 \\
0,0264 & $1,278 \times 10^{-14}$ & 0,1105 \\
0,0233 & $1,080 \times 10^{-14}$ & 0,2767 \\
\hline
\end{tabular}

Tabla 6.3: Coeficientes de difusión estimados para $0,0233 \leqslant \gamma \leqslant 0,1$. En el rango $0,0545 \leqslant \gamma \leqslant 0,1$ los ajustes fueron hechos en el intervalo $\mathrm{N}_{\text {iter }} \in\left(0 ; 10^{7}\right)$. Para $\gamma=0,0233$ y $\gamma=0,0483$, se extendió el cálculo al intervalo $\mathrm{N}_{\text {iter }} \in\left(0 ; 10^{8}\right)$.

$\delta=0$, donde puede advertirse que existen numerosas resonancias de alto orden que cruzan a la resonancia $1: 1$. Este punto es el que se encuentra más cerca del cruce de resonancias en la esquina inferior izquierda de la Fig. 5.3.

Los parámetros del sistema utilizados para estos experimetos son los mismos que los utilizados para los cálculos de la Sección 6.3. En cuanto a las condiciones iniciales, también se ha elegido un conjunto de $\mathrm{N}_{\mathrm{ci}}=10^{3}$ valores cercanos a $(\sqrt{3}, \sqrt{3})$ y dispuestos en una línea recta de longitud $\delta \rho=10^{-9}$ en dirección perpendicular al sentido de la resonancia. 

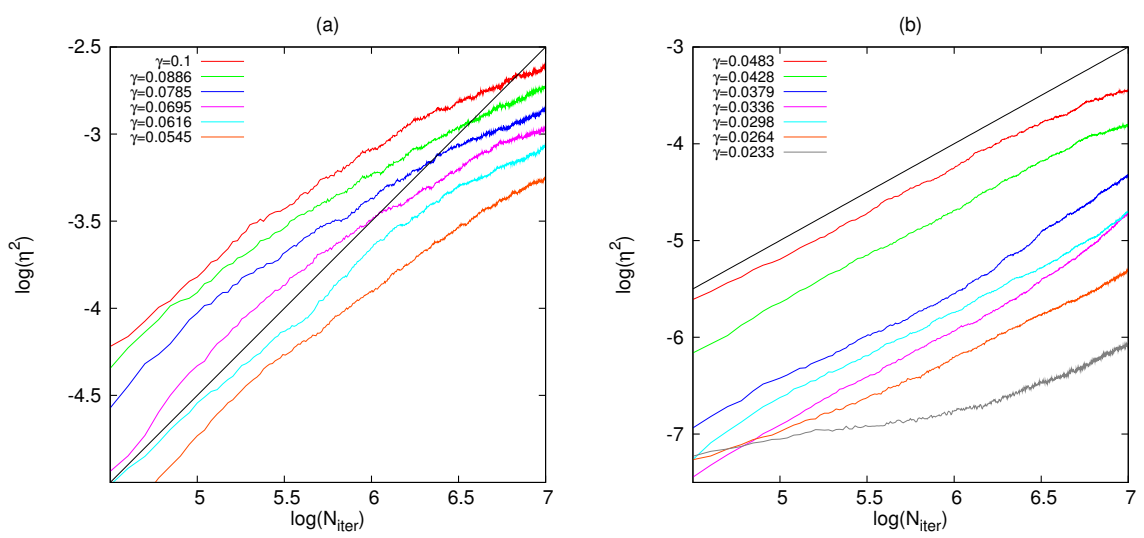

Figura 6.23: Evolución de $\eta^{2}$ con respecto al número de iteraciones, en escala logarítmica, para la forma normal centrada en $y_{1}^{*}=y_{2}^{*}=\sqrt{3}$ para distintos valores de $\gamma$ : (a) $0,0545 \leqslant \gamma \leqslant 0,1$ y (b) $0,0233 \leqslant \gamma \leqslant 0,0483$.

Las curvas de evolución de $\log \left(\eta^{2}\right)$ para los valores de $\gamma$ seleccionados se han dividido en dos grupos (Fig. 6.23) de acuerdo a su tipo de comportamiento, que coinciden con los grupos que resultaron en la Sección 6.3, a excepción de $\gamma=0,0233$, el cual presenta una evolución más lenta que el resto, no pudiendo alcanzar un régimen de difusión normal. En el panel (a) se muestran las curvas para el primer grupo de valores de $\gamma$, correspondiente a $0,0545 \leqslant \gamma \leqslant 0,1$. Si las comparamos con la recta identidad, podemos ver claramente que sus comportamientos no siguen una tendencia lineal. Recordemos de la Sección 6.3 que, si suponemos que la d.c.m. obedece una ley de potencias, entonces su evolución en escala logarítmica presentará una dependencia lineal, cuya pendiente $\beta$ nos indica la potencia de esa ley. Por ende, las evoluciones de $\eta^{2}$ correspondientes a este rango de valores de $\gamma$ presentan un tipo de difusión que no sigue una ley de potencias.

Este comportamiento puede entenderse mediante la Fig. 6.24, donde hemos escogido la evolución correspondiente a $\gamma=0,1$ y hemos representado la superficie de sección de las órbitas con un umbral de $10^{-3}$. El panel (a) corresponde a considerar $N_{i t e r}=10^{6}$ iteraciones. Podemos ver que la mayoría de los puntos que se encuentran representados en la figura están confinados a la resonancia $1: 1$, aunque existen algunos puntos que ya aparecen fuera de esta región, sobre las otras resonancias. En el panel (b), donde se representan los puntos considerando $\mathrm{N}_{\text {iter }}=10^{7}$ iteraciones, podemos ver que una gran parte de estas órbitas se encuentran difundiendo por otras resonancias. Estos puntos se encuentran muy alejados de la resonancia como para considerar que su aparición en la gráfica es debido a haber considerado una región de espesor no nulo, como se hizo en la Sección 6.3.1. Por ende, la difusión no ocurre solamente en la 
dirección de la resonancia $1: 1$, sino que tiene componentes en las direcciones de las otras resonancias también. Esto hace que disminuyan las componentes en la dirección de la resonancia $1: 1$, dando como resultado un aumento de $\eta^{2}$ a un ritmo más lento que para el caso de una resonancia simple.
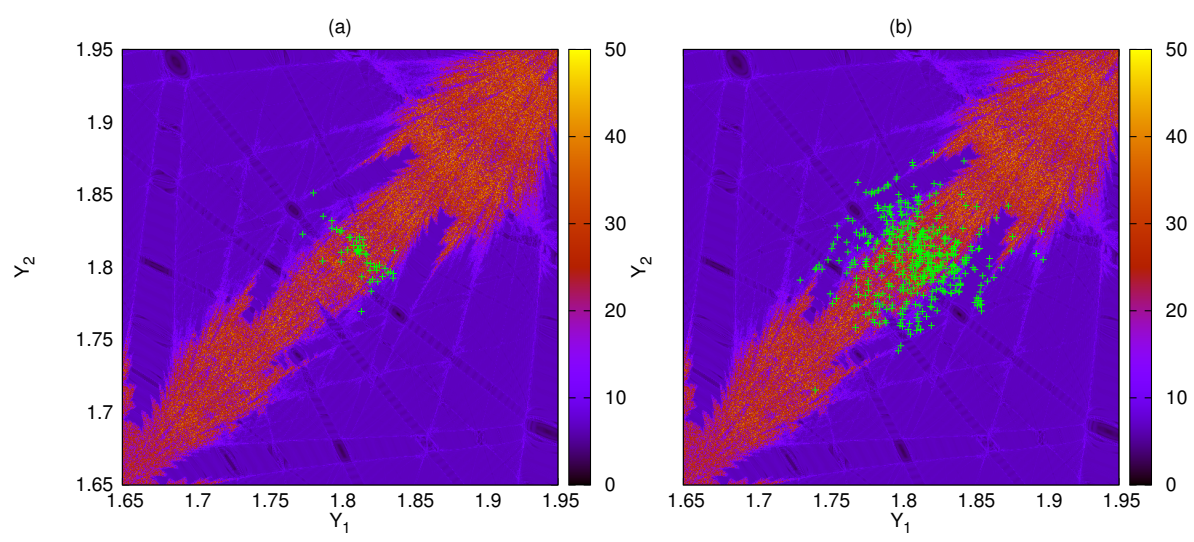

Figura 6.24: Superficie de sección $\left(\sqrt{x_{1}^{2}+x_{2}^{2}} \leqslant 10^{-3}\right)$ en el plano $\left(y_{1}, y_{2}\right)$ del conjunto de órbitas correspondiente al mapa con $\gamma=0,1$ para (a) $\mathrm{N}_{\text {iter }}=10^{6} \mathrm{y}$ (b) $\mathrm{N}_{\text {iter }}=10^{7}$.

En cuanto al segundo conjunto de valores de $\gamma$, reflejados en el panel (b) de la Fig. 6.23, la evolución de $\eta^{2}$ presenta un comportamiento aproximadamente lineal, con pendientes cercanas a la unidad. En la Fig. 6.25 se muestra la superficie de sección para $\gamma=0,0483$ (panel (a)) y $\gamma=0,0264$ (panel (b)), ambas considerando $\mathrm{N}_{\text {iter }}=10^{7}$. Podemos ver que los puntos en ambos escenarios permanecen confinados a las cercanías de la resonancia, estando en acuerdo con el crecimiento lineal mencionado anteriormente.

Debido a que las evoluciones para este rango de valores de $\gamma$ presentan una dependencia lineal, podemos estimar su pendiente. Los resultados obtenidos, junto con el error en su estimación $\left(E_{\beta}\right)$ son reflejados en el panel izquierdo de la Tabla 6.4. Vemos que todos los valores de $\beta$ estimados aquí pueden ser considerados cercanos a la unidad y han sido estimados con un error considerablemente bajo $\left(\mathrm{E}_{\beta}<0,4 \%\right)$, por lo que es factible la estimación de un coeficiente de difusión, los cuales se muestran en el panel derecho de la Tabla 6.4 junto con su error $E_{\mathcal{D}}$.

A pesar de que la cercanía con el cruce de resonancias hace que se incremente la perturbación, para el rango de valores $0,01 \leqslant \gamma \leqslant 0,0233$ se han obtenido valores de $\beta$ muy pequeños como para poder considerarlos en un régimen de difusión normal. Por ende, han sido excluidos del estudio. 

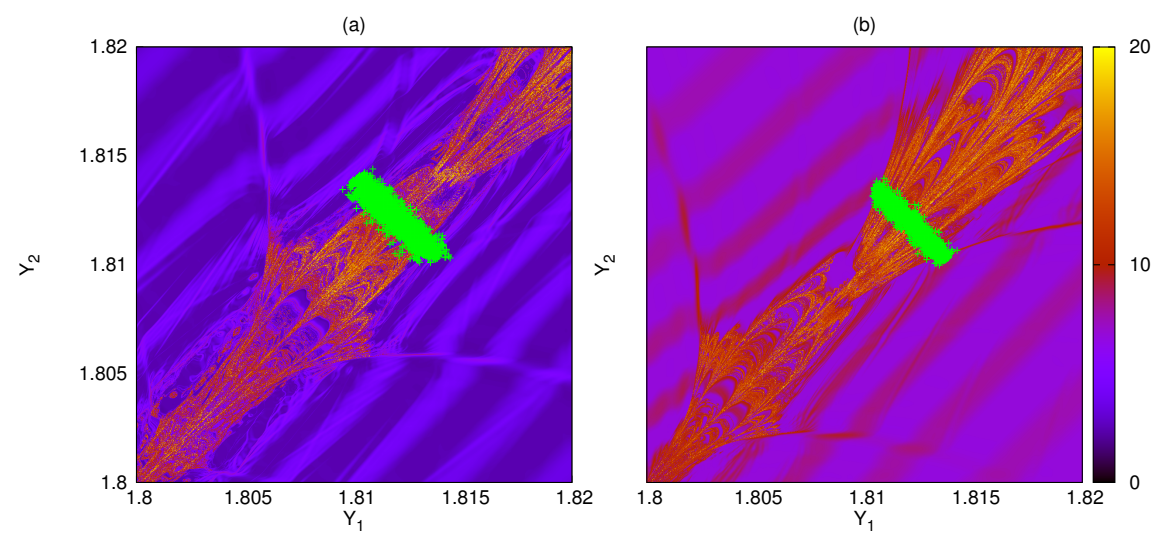

Figura 6.25: Superficie de sección $\left(\sqrt{x_{1}^{2}+x_{2}^{2}} \leqslant 10^{-3}\right)$ en el plano $\left(y_{1}, y_{2}\right)$ del conjunto de órbitas correspondiente al mapa con $\gamma=0,0483$ (a) y $\gamma=0,0264$ (b) para $\mathrm{N}_{\text {iter }}=10^{7}$.

\begin{tabular}{|c|c|c|}
\hline$\gamma$ & $\beta$ & $E_{\beta}(\%)$ \\
\hline \hline 0,0483 & 0,851 & 0,2675 \\
0,0428 & 0,926 & 0,2344 \\
0,0379 & 1,132 & 0,271 \\
0,0336 & 1,126 & 0,3468 \\
0,0298 & 0,978 & 0,215 \\
0,0264 & 0,853 & 0,1912 \\
\hline
\end{tabular}

\begin{tabular}{|c|c|c|}
\hline$\gamma$ & $\mathcal{D}$ & $\mathrm{E}_{\mathcal{D}}(\%)$ \\
\hline \hline 0,0483 & $3,543 \times 10^{-11}$ & 0,5229 \\
0,0428 & $1,599 \times 10^{-11}$ & 0,5026 \\
0,0379 & $4,848 \times 10^{-12}$ & 0,2426 \\
0,0336 & $1,879 \times 10^{-12}$ & 0,5438 \\
0,0298 & $1,890 \times 10^{-12}$ & 0,3164 \\
0,0264 & $4,644 \times 10^{-13}$ & 0,1489 \\
\hline
\end{tabular}

Tabla 6.4: Panel izquierdo: Pendiente $\beta$ y error $E_{\beta}$ del ajuste $\log \left(\eta^{2}\right)=\alpha+\beta \log \left(N_{\text {iter }}\right)$ para $N_{\text {iter }}=10^{7}$ en el rango $0,0264 \leqslant \gamma \leqslant 0,0483$, donde $\alpha$ y $\beta$ son parámetros a determinar. Los ajustes fueron realizados en el intervarlo $N_{\text {iter }} \in\left(10^{5} ; 10^{7}\right)$. Panel derecho: Coeficientes de difusión $\mathcal{D}$ junto con el error $E_{\mathcal{D}}$ estimados en el intervarlo $N_{i t e r} \in\left(10^{5} ; 10^{7}\right)$, para el rango $0,0264 \leqslant \gamma \leqslant 0,0483$.

Con estos resultados podemos concluir que, para valores suficientemente pequeños de $\gamma$, la influencia de las resonancias que atraviesan la resonancia $1: 1$ es despreciable, y por ende, podemos emplear el desarrollo de las formas normales para una resonancia simple.

Cabe destacar que este grupo de valores de $\gamma$ manifestó un comportamiento difusivo aproximadamente normal para $\mathrm{N}_{i t e r} \in\left(0 ; 10^{7}\right)$. En cambio, cuando se consideró el mismo grupo de valores de $\gamma$, pero ubicados lejos del cruce de resonancias, este tiempo no fue suficiente para que manifestaran difusión normal, 
necesitando realizar $\mathrm{N}_{\mathrm{iter}}=10^{8}$ iteraciones. Continuando con la hipótesis propuesta para el primer grupo, la cercanía con el cruce hace que la perturbación sea mayor que en el caso de la Sección 6.3.2, haciendo que la difusión normal se manifieste en un número de iteraciones menor.

\subsection{DIscusión}

En este capítulo se estudió la difusión caótica en el espacio de las acciones para el CRSSM a través de dos experimentos, cuyas condiciones iniciales se encuentran sobre la resonancia $1: 1$.

El primer experimento consistió en cuantificar la difusión en las vecindades del punto $y_{1}^{*}=y_{2}^{*}=\sqrt{3}+\delta$ con $\delta=0,08$. Este valor de $\delta$ se escogió de manera de encontrarse lo suficientemente lejos de un cruce de resonacias como para que el desarrollo en formas normales para una resonancia simple tenga validez. Se calculó la evolución de la d.c.m. $\left(\eta^{2}\right)$ con respecto al número de iteraciones $\mathrm{N}_{\text {iter }}$ para distintos valores del parámetro perturbativo $\gamma$. Para los valores de $\gamma$ en los que la variación secular quedó oculta por la amplitud de las oscilaciones, se calculó también la d.c.m. en el mismo conjunto de variables considerando solamente los puntos pertenecientes a la región $\left|x_{1}\right|+\left|x_{2}\right| \leqslant 0,1$, junto con el cálculo de la d.c.m. en el nuevo conjunto de variables. Se pudo concluir que, si bien ambas técnicas reducen la amplitud de las oscilaciones, la transformación a las formas normales resultó ser mucho más efectiva, además de ser válida para valores muy pequeños del parámetro perturbativo $\gamma$.

Basándonos en la hipótesis de que la dependencia entre $\eta^{2}$ y $N_{i t e r}$ es una ley de potencias, se realizaron cálculos de la evolución de la d.c.m., siguiendo una escala logarítmica, para estimar su pendiente en un intento de detectar si, en las regiones consideradas, el comportamiento difusivo se puede aproximar como normal. Se encontró que, en el rango $0,0545 \leqslant \gamma \leqslant 0,1$, la pendiente obtenida del ajuste lineal de la evolución de $\log \left(\eta^{2}\right)$ con respecto a $\log \left(\mathrm{N}_{\text {iter }}\right)$ para $\mathrm{N}_{\text {iter }} \in\left(0 ; 10^{7}\right)$ es cercana a la unidad, por lo que puede suponerse que la difusión es normal. En dos de estos escenarios (correspondientes a $\gamma=0,0695$ y $\gamma=0,0616$ ) el comportamiento encontrado no fue posible aproximarlo como un comportamiento lineal, quizá debido a efectos como la presencia de órbitas tipo sticky, que provocan una disminución de la velocidad con la que difunde el conjunto de condiciones iniciales. Para estos dos escenarios, se reiteró el cálculo, pero considerando un número mayor de iteraciones, $N_{i t e r}=\left(0 ; 10^{8}\right)$. En esta configuración ambas evoluciones de la d.c.m. presentaron un comportamiento cercano al lineal.

El segundo grupo de valores de $\gamma, 0,0233 \leqslant \gamma \leqslant 0,0483$, presentó una evolución de las curvas de d.c.m. más lenta que las del primer grupo, necesitando del cálculo de $\eta^{2}$ para $N_{i t e r} \in\left(0 ; 10^{8}\right)$. Para este número de iteraciones, todas 
las curvas presentaron un comportamiento aproximadamente lineal, por lo que se supuso que se encuentran en un régimen de difusión normal.

Para el último grupo de valores considerados, $0,01 \leqslant \gamma \leqslant 0,0207$, la perturbación es tan pequeña que el cálculo realizado para $10^{8}$ iteraciones no fue suficiente para evidenciar difusión normal. Para este conjunto de valores se concluyó que es necesario realizar el cálculo para un número aún mayor de iteraciones.

Para los valores de $\gamma$ que presentaron difusión normal, se analizó el comportamiento de la evolución de la d.c.m. con respecto al número de iteraciones en una escala lineal. Esto se hizo para estimar el coeficiente de difusión $\mathcal{D}$ a través de un ajuste lineal de la curva de evolución. La estimación de $\mathcal{D}$ dio valores en el rango de $2,131 \times 10^{-10}$ para $\gamma=0,1$ y $N_{\text {iter }}=10^{7}$ hasta $1,080 \times 10^{-14}$ para $\gamma=0,0233$ y $\mathrm{N}_{\text {iter }}=10^{8}$.

Para comprender mejor la relevancia de los resultados obtenidos en la estimación de $\mathcal{D}$ pensemos en lo siguiente. A partir de la Ec. (4.5) podemos despejar el tiempo, obteniendo $t=\frac{\eta^{2}}{\mathcal{D}}$. Esta expresión significa que, una vez que obtenemos el valor de $\mathcal{D}$ podemos estimar el tiempo necesario para que la acción efectúe una variación cuadrática media $\eta^{2}$. Por ejemplo, en el estudio de la dinámica de un asteroide, podríamos considerar que los valores típicos de excentricidad se encuentran en el rango $e \sim 10^{-2}-10^{-1}$. Consideremos ahora que una variación $\Delta e \approx 10^{-1}$ podría provocar que la dinámica del asteroide cambie (por ejemplo, saliendo de la resonancia en que se encuentra). Su $\eta^{2}$ correspondiente será $10^{-2} \mathrm{y}$, el tiempo necesario para que e experimente dicha variación, considerando los valores de $\mathcal{D}$ obtenidos en la Tabla 6.3 , será $t \approx 5 \times 10^{7}$ períodos para $\gamma=0,1 \mathrm{y} \mathrm{t} \approx 10^{12}$ para $\gamma=0,0233$, donde un período característico del sistema puede ser $T \sim 10$ años. Luego vemos que para los valores más grandes del parámetro perturbativo $\gamma$, el efecto ocurre en la escala de la edad del Sistema Solar, mientras que para los valores inferiores de $\gamma$ el tiempo excede a dicha edad.

Finalmente, la relevancia de los valores obtenidos para $\mathcal{D}$ dependerán fuertemente del tamaño en que las acciones variarán, cambiando, por consiguiente, la escala de tiempo necesaria para que ésta se manifieste.

El segundo experimento se realizó considerando un punto central ubicado sobre la misma resonancia principal que el experimento anterior, pero en una región cercana a un cruce de resonancias para estudiar cómo afecta ésta a la evolución de la difusión. Este punto corresponde a considerar $y_{1}^{*}=y_{2}^{*}=\sqrt{3}+\delta$, con $\delta=0$. Se analizaron los mismos valores de $\gamma$ que presentaron difusión normal en el primer experimento en el intervalo $\mathrm{N}_{\text {iter }} \in\left(0 ; 10^{7}\right)$. Para el grupo de valores del parámetro perturbativo $0,0545 \leqslant \gamma \leqslant 0,1$, la d.c.m. presentó un comportamiento no lineal. Esto podría ser debido a que, por la cercanía con un cruce de resonancias, la perturbación es mayor y podría favorecer a la superpo- 
sición de un gran número de resonancias de alto orden. Esto podría provocar que una parte considerable de las órbitas hayan difundido por otras resonancias, disminuyendo la velocidad de la difusión a lo largo de la resonancia $1: 1$. En cuanto al segundo conjunto de valores, $0,0264 \leqslant \gamma \leqslant 0,0483$, sus evoluciones sí siguieron una tendencia lineal, con pendientes cercanas a la unidad. Para este conjunto se determinaron sus coeficientes de difusión. Los valores de $\mathcal{D}$ para este intervalo se encuentran en el rango de $3,543 \times 10^{-11}$ para $\gamma=0,0483$ hasta $4,644 \times 10^{-13}$ para $\gamma=0,0264$. Por lo tanto concluimos que, en el caso de escoger un punto central cercano a un cruce de resonancias, debemos escoger valores de $\gamma$ más pequeños que en un escenario donde el punto central $\left(y_{1}^{*}, y_{2}^{*}\right)$ se encuentre lejos de un cruce, de manera que las perturbaciones producidas por el cruce sean despreciables. De esta manera es posible emplear el desarrollo de formas normales correspondiente a una resonancia simple, hasta valores de $\gamma$ lo suficientemente pequeños como para que la amplitud de las oscilaciones haga que no sea posible cuantificar la difusión. 


\section{CONCLUSIONES}

El objetivo que dio origen a este trabajo de tesis fue realizar el estudio de la difusión para un sistema hamiltoniano discreto, es decir, un mapa simpléctico. Se abordó este problema de una manera empírica, a través del cálculo numérico de conjuntos de condiciones iniciales ubicadas en una región muy pequeña del espacio de fases, en particular en la capa estocástica de una resonancia simple, y siguiendo su evolución temporal. La elección de la ubicación inicial del conjunto fue mediante la previa detección de las regiones del espacio de acciones del mapa escogido (el CRSSM) que presentaran un comportamiento caótico.

Un hecho para destacar de este trabajo de tesis fue que hemos desarrollado nuestras propias herramientas para el estudio y cuantificación de la difusión, basándonos en técnicas halladas en la literatura, como los indicadores de caos descriptos en el Capítulo 2 o la transformación a las formas normales, como las utilizadas en [30] para un mapa 2D, descripta en el Capítulo 4. En el presente trabajo pueden distinguirse claramente dos partes: la primera de ellas está abocada al desarrollo de la herramienta que calcula los ICs, el LP-VIcode, mientras que la otra está dedicada al diseño de un método que permite realizar la construcción de la transformación a formas normales del sistema, para luego poder caracterizar la difusión.

Dado que al final de los capítulos que conforman este trabajo hemos incluido sus correspondientes discusiones, aquí simplemente haremos un resumen de los principales resultados obtenidos.

Como ya hemos mencionado, es necesario primero conocer las regiones de regularidad y caoticidad que presenta el sistema, para tener información más detallada de su estructura dinámica. Para ello hemos desarrollado un código que implementa el cálculo de doce indicadores variacionales de caos.

En la primera parte de este trabajo de tesis, en el Capítulo 2, comenzamos con la definición de algunos conceptos básicos que serían luego necesarios para la comprensión del resto del trabajo. Luego, continuamos con la introducción de los indicadores variacionales implementados en el LP-VIcode, enunciando 
sus principales características que lo definen y describiendo cómo se han implementado en el código.

En el Capítulo 3 presentamos el LP-VIcode, describiendo sus principales características. Mostramos cómo opera el programa principal, cómo funciona la entrada y salida de datos, para luego describir cómo se organizaron los cálculos de los ICs, agrupándolos en unidades. Seguido de esto mostramos cómo están conformadas las unidades, justificando la inclusión de cada uno de los ICs en una dada unidad.

Una vez introducido el LP-VIcode, se mostraron algunos resultados obtenidos, como la medición de los tiempos de CPU para el cálculo de los distintos ICs y de las distintas unidades. Estos resultados revelaron una disminución de aproximadamente $35 \%$ del tiempo de cómputo empleando el LP-VIcode, con respecto al tiempo empleado en el cálculo, por separado, de todos los indicadores.

Junto con este estudio se realizó el cálculo de los ICs propiamente dichos, tanto para la versión de mapas como para la de flujos hamiltonianos. Para la versión de mapas se empleó un mapa simpléctico 4D, mientras que para flujos hamiltonianos se empleó el modelo de Hénon y Heiles. En ambos sistemas se escogieron condiciones iniciales utilizadas en la literatura, correspondientes a órbitas cuya naturaleza (regular o caótica) ya ha sido determinada correctamente por otros autores. Con los resultados obtenidos, y su comparación con los publicados por otros autores, pudimos ilustrar la correcta implementación de los ICs en el LP-VIcode.

En cuanto al estudio de la difusión caótica hemos visto que, para perturbaciones muy pequeñas, no es posible detectar la difusión debido a la significativa amplitud de las oscilaciones que introduce el conjunto de variables utilizado. Por ello hemos tenido que recurrir a distintas técnicas. Una de ellas consiste en tomar los puntos del espacio de las acciones que se encuentran cerca de una dada superficie de sección, por ejemplo, $\left|x_{1}\right|+\left|x_{2}\right|=0$. Empleando esta técnica se ha logrado reducir la amplitud de las oscilaciones, facilitando la detección de la variación secular de las acciones. A partir de un cierto valor en la perturbación, la amplitud de las oscilaciones vuelve a cobrar importancia. Para vencer este obstáculo resultó conveniente calcular la transformación a las formas normales. No existía hasta el momento una versión para mapas de esta técnica, por lo que fue desarrollada en este trabajo.

En la segunda parte de esta tesis comenzamos con el Capítulo 4, que fue dedicado a la explicación detallada de cómo construir la transformación a la forma normal de nuestro mapa. Se ha utilizado para este fin el desarrollo de la transformación a formas normales para una resonancia simple. La explicación incluida en este capítulo detalla cómo expresar nuestro mapa original como un desarrollo de Fourier. Luego se introduce el parámetro de contabilidad, que 
es la piedra angular sobre la cual se apoya el método para construir la transformación. Continuamos con la obtención de la transformación buscada y, por último, la construcción de la transformación inversa, para poder expresar las variables nuevas en función de las originales, y así poder calcular las nuevas coordenadas en el espacio de fases.

El Capítulo 5 se encuentra dividido en dos partes. En la primera parte explicamos cómo aplicar el mecanismo desarrollado y presentado en el Capítulo 4 a un mapa 4D en particular. En este trabajo fue escogido el CRSSM. Para esto hemos construido un código, el cual es presentado en este capítulo. Presentamos este código, desarrollado en Fortran 90, y hemos descripto sus principales características. Se encuentra explicado en detalle en el Apéndice C. La segunda parte de este capítulo está dedicada a mostrar los resultados que surgen de la aplicación del código a nuestro problema. Se midieron los tiempos de CPU necesarios para el cálculo de las transformaciones, por un lado, y para el cálculo de la d.c.m., por otro. Con estos resultados se pudieron proponer relaciones entre los tiempos y el orden del desarrollo. Luego de esto se estimó también el orden óptimo del desarrollo para dos regiones pertenecientes a la resonancia 1 : 1. Este orden se estimó de forma empírica, analizando la evolución de la d.c.m. para los distintos órdenes.

En la primera de estas regiones, lejos de cruces de resonancias, se estableció un orden óptimo $r=7$ para todos los valores de $\gamma$. En cuanto a la otra región, más cercana a un cruce de resonancias, no fue posible determinar un orden óptimo, presumiblemente debido a que la proximidad con otras resonancias de alto orden afectan al desarrollo de la forma normal. Se escogió, para mantener uniformidad con la primera región, el orden $r=7$.

Por último, en el Capítulo 6 se presentaron los resultados de la cuantificación de la difusión para un rango de valores del parámetro perturbativo $\gamma$ $(0,01 \leqslant \gamma \leqslant 0,1)$. Este estudio tuvo como objetivo la búsqueda de intervalos de tiempo en los que la difusión puede aproximarse a un régimen normal. Para estos intervalos, luego, se estimó el coeficiente de difusión $\mathcal{D}$. El primer conjunto de condiciones iniciales elegido fue un ensamble ubicado en una región alejada de un cruce de resonancias. Estos cálculos arrojaron como resultado que, para los valores más grandes de $\gamma(0,0545 \leqslant \gamma \leqslant 0,1)$, en un tiempo de $\mathrm{N}_{\text {iter }}=10^{7}$, las curvas de la evolución de la d.c.m. presentaron un comportamiento difusivo cercano a la difusión normal. Para el rango de valores intermedios de $\gamma$ $(0,0233 \leqslant \gamma \leqslant 0,0483)$, el tamaño de la perturbación hizo que este tiempo no sea suficiente para que se manifieste un comportamiento difusivo normal. Por ello se realizaron nuevamente los cálculos para $\mathrm{N}_{i t e r}=10^{8}$, logrando manifestar, en este escenario, un comportamiento difusivo aproximadamente normal. Para el rango inferior de valores de $\gamma(0,01 \leqslant \gamma \leqslant 0,0207)$, la perturbación fue demasiado pequeña como para que experimente una difusión normal. Dado 
que considerar un número $\mathrm{N}_{\mathrm{iter}}$ mayor no es físicamente realista, no se ha continuado con su investigación.

Para el rango de valores de $\gamma$ que presentaron un comportamiento difusivo que pudo aproximarse a un régimen normal, se han estimado sus coeficientes de difusión, obteniendo valores que cubren el rango $\mathcal{D}=2,131 \times 10^{-10}$ para $\gamma=0,1$ y $N_{\text {iter }}=10^{7}$ hasta $\mathcal{D}=1,08 \times 10^{-14}$ y $N_{i t e r}=10^{8}$ para $\gamma=0,0233$.

Luego del estudio para una resonancia simple, se realizó el mismo estudio para una región cercana a un cruce de resonancias. Este experimento arrojó que, para los valores mayores de $\gamma(0,0545 \leqslant \gamma \leqslant 0,1)$, las condiciones iniciales se dispersan por otras resonancias de alto orden que cruzan la resonancia $1: 1$. Para los valores intermedios de $\gamma(0,0264 \leqslant \gamma \leqslant 0,0483)$, sus trayectorias no se ven afectadas por la cercanía con el cruce de resonancias y allí se pudo estimar el valor de $\mathcal{D}$, obteniendo valores $3,543 \times 10^{-11} \leqslant \mathcal{D} \leqslant 4,644 \times 10^{-13}$.

Como conclusión final de este trabajo tenemos:

- La elaboración de un código, el LP-VIcode, para el cómputo de ICs. Este programa nos permite el cálculo de una gran cantidad de indicadores variacionales, dando la libertad de elegir cuáles de éstos calcular de forma simultánea, de acuerdo al problema que se desea abordar.

- La comprensión y el diseño de un método que calcula la tranformación a formas normales, junto con el desarrollo de un mecanismo para su construcción, y la elaboración de un código que realice su cálculo, para un mapa simpléctico 4D del tipo cuasi-torsional. Este método fue posible implementarlo gracias a la introducción de una técnica de ordenamiento de los términos de Fourier según su tamaño. Este código nos permite realizar la construcción, para un orden $\mathrm{N}$, de la transformación, el nuevo mapa, la transformación inversa y el cálculo de la desviación cuadrática media en la dirección de la resonancia a través de la obtención de las nuevas coordenadas en el espacio de fases en función de las coordenadas originales.

Si bien ambos programas se encuentran en una etapa funcional, en la cual son capaces de calcular la evolución de los ICs, por un lado, y la forma normal junto con la evolución de la d.c.m. por otro, es posible seguir trabajando sobre ellos y construir versiones más robustas, estables y eficientes.

Como trabajo a futuro, con respecto a la parte numérica, nos hemos propuesto lo siguiente:

- Con respecto al LP-VIcode, se está desarrollando una nueva versión, que optimiza el cálculo de los indicadores. Esta optimización se llevará a cabo 
a través de una mejor comprensión de las ecuaciones que comparten, de manera de reducir al mínimo el número de operaciones.

Otra mejora será reescribir la rutina integradora, de manera que el código resulte independiente del algoritmo integrador.

- En cuanto a la transformación a formas normales utilizada en la segunda parte de este trabajo de tesis, nos propusimos estudiar un mecanismo para generalizar esta técnica y, en consecuencia su implementación numérica, para extender la construcción de la forma normal de un mapa simpléctico de $2 \mathrm{~N}$ dimensiones. También se estudiará la factibilidad de llevar a cabo la creación de una rutina que automatice el proceso de construcción del desarrollo en series de Fourier sobre el mapa original.

Concerniente al estudio de la difusión, como trabajo a futuro queremos extender el análisis de la d.c.m. a diferentes regiones de la resonancia $1: 1$, ya sean regiones más cercanas a un cruce, o cercanas a cruces de diferente magnitud y estudiar cómo ésto afecta a la naturaleza normal de la difusión estimada en este trabajo. Nos proponemos también profundizar las investigaciones sobre el carácter de los distintos regímenes (subdifusivo, normal, superdifusivo) y procurar establecer si, efectivamente, al aumentar progresivamente el tiempo de cómputo, dentro de un régimen de perturbaciones pequeñas, la difusión resulta ser universalmente normal, es decir, si el carácter normal se manifiesta independientemente de qué tan grande sea el intervalo tiempo considerado. También queremos extender el estudio realizado en este trabajo (y los propuestos aquí) a regiones pertenecientes a otras resonancias, ya sean del mismo orden o superiores, en un intento de tener un mejor conocimiento de la posible dependencia de la tasa de difusión con el orden de la resonancia.

Un objetivo adicional será la aplicación de esta técnica a sistemas más realistas, como por ejemplo, un sistema planetario, para poder estudiar fenómenos como la estabilidad a largo plazo del sistema. 



\section{BIBLIOGRAFÍA}

[1] R. Barrio. Sensitivity tools vs Poincaré sections. Chaos, Solitons and Fractals, 25:711-726, 2005.

[2] R. Barrio, F. Blesa y S. Serrano. Qualitative analysis of the Rössler equations: Bifurcations of limit cycles and chaotic attractors. Physica D: Nonlinear Phenomena, 238:1087-1100, 2009.

[3] R. Barrio, W. Borczyk y S. Breiter. Spurious structures in chaos indicators maps. Chaos, Solitons and Fractals, 40:1697-1714, 2009.

[4] R. Barrio, F. Blesa y S. Serrano. Bifurcations and chaos in Hamiltonian systems. International Journal of Bifurcation and Chaos, 20:1293-1319, 2010.

[5] A. Bazzani, S. Marmi y G. Turchetti. Nekhoroshev estimate for isochronous non resonant symplectic maps. Celestial Mechanics and Dynamical Astronomy, 47:333-359, 1990.

[6] G. Benettin, L. Galgani y J.M. Strelcyn. Kolmogorov entropy and numerical experiments. Physical Review A, 14:2338-2345, 1976.

[7] G. Benettin, L. Galgani, A. Giorgilli y J.M. Strelcyn. Lyapunov characteristic exponents for smooth dynamical systems; a method for computing all of them. Meccanica, 15:Part I: Theory, 9-20; Part II: Numerical Applications, 21-30, 1980.

[8] J. Binney y D. Spergel. Spectral stellar dynamics. Astrophysical Journal, 252: 308-321, 1982.

[9] J. Binney y D. Spergel. Spectral stellar dynamics. ii - the action integrals. Monthly Notices of the Royal Astronomical Society, 206:159-177, 1984.

[10] F. Cachucho, P. Cincotta y S. Ferraz-Mello. Chirikov diffusion in the asteroidal three-body resonance $(5,-2,-2)$. Celestial Mechanics and Dynamical Astronomy, 108:35-58, 2010.

[11] B. Chirikov. Research concerning the theory of non-linear resonance and stochasticity. Institute of Nuclear Physics (Traducido al inglés por CERN (1971), Novosibirsk, Rusia, preprint $n 267$ edition, 1969.

[12] B. Chirikov. A universal instability of many-dimensional oscillator systems. Physics Reports, 52:263-379, 1979. 
[13] P. Cincotta. Arnold diffusion: An overview through dynamical astronomy. New Astronomy Reviews, 46:13-39, 2002.

[14] P. Cincotta y Giordano. International Journal of Bifurcation and Chaos, In press., 2012.

[15] P. Cincotta y C. Giordano. Diffusion in multidimensional Hamiltonian systems. En: P. Cincotta, C. Giordano \& C. Efthymiopoulos eds.: 3rd La Plata International School on Astronomy and Geophysics "Chaos, Diffusion and Non-integrability in Hamiltonian Systems-Application to Astronomy.", 1:185216, 2012.

[16] P. Cincotta y C. Simó. Simple tools to study global dynamics in nonaxisymmetric galactic potentials -i. Astronomy $\mathcal{E}$ Astrophysics Supplement, 147:205-228, 2000.

[17] P. Cincotta, C. Giordano y C. Simó. Phase space structure of multidimensional systems by means of the mean exponential growth factor of nearby orbits. Physica D: Nonlinear Phenomena, 182:151-178, 2003.

[18] P. Cincotta, Ch. Efthymiopoulos, C. Giordano y M. Mestre. Chirikov and Nekhoroshev diffusion estimates: bridging the two sides of the river. Physica D: Nonlinear Phenomena, 266:49-64, 2014.

[19] G. Contopoulos y A. Giorgilli. Bifurcations and complex instability in a 4-dimensional symplectic mapping. Meccanica, 23:19-28, 1988.

[20] G. Contopoulos y N. Voglis. Spectra of stretching numbers and helicity angles in dynamical systems. Celestial Mechanics and Dynamical Astronomy, 64:1-20, 1996.

[21] R. Cordeiro. Anomalous diffusion in the asteroid belt. The Astronomical Journal, 132:2114-2126, 2006.

[22] R. Cordeiro y L. Mendes de Souza. Anomalous diffusion in the first order Jovian resonance. Astronomy \& Astrophysics, 439:375-385, 2005.

[23] L. Darriba, N. Maffione, P. Cincotta y C. Giordano. Chaos detection tools: The LP-VIcode and its applications. En: P. Cincotta, C. Giordano \& C. Efthymiopoulos eds.: 3 rd La Plata International School on Astronomy and Geophysics "Chaos, Diffusion and Non-integrability in Hamiltonian Systems-Application to Astronomy.", 1:345-366, 2012.

[24] L. Darriba, N. Maffione, P. Cincotta y C. Giordano. Comparative study of variational chaos indicators and ODEs' numerical integrators. International Journal of Bifurcation and Chaos, 22:10-33, 2012. 
[25] Ch. Efthymiopoulos. Formal integrals and Nekhoroshev stability in a mapping model for the Trojan asteroids. Celestial Mechanics and Dynamical Astronomy, 92:29-52, 2005.

[26] Ch. Efthymiopoulos. On the connection between the Nekhoroshev theorem and Arnold diffusion. Celestial Mechanics and Dynamical Astronomy, 102:49-68, 2008.

[27] Ch. Efthymiopoulos. Canonical perturbation theory, stability and diffusion in Hamiltonian systems: Applications to dynamical astronomy. Proceedings of the 3rd La Plata School on Astronomy and Geophysics, WSAAA, pages 3-143, 2013.

[28] Ch. Efthymiopoulos. High order normal form stability estimates for coorbital motion. Celestial Mechanics and Dynamical Astronomy, 117:101-112, 2013.

[29] Ch. Efthymiopoulos y M. Harsoula. The speed of Arnold diffusion. Physica D: Nonlinear phenomena, 251:19-38, 2013.

[30] Ch. Efthymiopoulos y Z. Sándor. Optimized Nekhoroshev stability estimates for the Trojan asteroids with a symplectic mapping model of co-orbital motion. Monthly Notices of the Royal Astronomical Society, 364:253-271, 2005.

[31] A. Einstein. On the movement of small particles suspended in a stationary liquid demanded by the molecular kinetic theory of heat, volume 17. Dover Publications, Nueva York, EE.UU., 1956.

[32] M. Fouchard, E. Lega, Ch. Froeschlé y Cl. Froeschlé. On the relationship between fast Lyapunov indicator and periodic orbits for continuous flows. Celestial Mechanics and Dynamical Astronomy, 83:205-222, 2002.

[33] Cl. Froeschlé. Numerical study of a 4d mapping. Astronomy $\mathcal{E}$ Astrophysics, 16:172-189, 1972.

[34] Cl. Froeschlé y E. Lega. On the structure of symplectic mappings. The fast Lyapunov indicator: a very sensitive tool. Celestial Mechanics and Dynamical Astronomy, 78:167-195, 2000.

[35] Cl. Froeschlé y E. Lega. The fine structure of Hamiltonian systems revealed using the fast Lyapunov indicator. En: B.A. Steves et al. (eds) Chaotic Worlds: from Order to Disorder in Gravitational N-Body Dynamical Systems, pages 131$165,2006$.

[36] Cl. Froeschlé, R. Gonczi y E. Lega. The fast Lyapunov indicator: a simple tool to detect weak chaos. Application to the structure of the main asteroidal belt. Planetary and Space Science, 45:881-886, 1997. 
[37] Cl. Froeschlé, E. Lega y R. Gonczi. Fast Lyapunov indicators. Application to asteroidal motion. Celestial Mechanics and Dynamical Astronomy, 67:41-62, 1997.

[38] Cl. Froeschlé, M. Guzzo y E. Lega. Local and global diffusion along resonant lines in discrete quasi-integrable dyamical systems. Celestial Mechanics and Dynamical Astronomy, 92:243-255, 2005.

[39] Cl. Froeschlé, E. Lega y M. Guzzo. Analysis of the chaotic behaviour of orbits diffusing along de Arnold web. Celestial Mechanics and Dynamical Astronomy, 95:141-153, 2005.

[40] C. Giordano y P. Cincotta. Chaotic diffusion of orbits in systems with divided phase space. Astronomy \& Astrophysics, 423:745-753, 2004.

[41] A. Giorgilli. Notes on exponential stability of Hamiltonian systems, in Dynamical Systems. Part I: Hamiltonian Systems and Celestial Mechanics, Pubblicazioni della Classe di Scienze, Scuola Normale Superiore, Pisa, 2002.

[42] M. Guzzo y E. Lega. The numerical detection of the Arnold web and its use for long-term diffusion studies in conservative and weakly dissipative systems. Chaos, 23:1-12, 2013.

[43] M. Guzzo, E. Lega y Cl. Froeschlé. On the numerical detection of the effective stability of chaotic motions in quasi-integrable systems. Physica $D, 163: 1-25,2002$.

[44] M. Guzzo, E. Lega y Cl. Froeschlé. First numerical evidence of global Arnold diffusion in quasi-integrable systems. Discrete and Continuous Dynamical Systems - Series B, 3:687-698, 2005.

[45] M. Hénon y C. Heiles. The applicability of the third integral of motion: some numerical experiments. Astronomical Journal, 1:73-79, 1964.

[46] T. Konishi y K. Kaneko. Diffusion in Hamiltonian chaos and its size dependence. Journal of Physics A: Mathematical and General, 23:L715-L720, 1990.

[47] J. Laskar. The chaotic motion of the Solar System - A numerical estimate of the size of the chaotic zones. Icarus, 88:266-291, 1990.

[48] J. Laskar. Frequency analysis for multi-dimensional systems. Global dynamics and diffusion. Physica D: Nonlinear Phenomena, 67:257-281, 1993.

[49] J. Laskar. Frequency map analysis and quasiperiodic decompositions. En: Proceedings of Porquerolles School, arXiv: math/0305364, 2003. 
[5o] E. Lega y Cl. Froeschlé. On the relationship between fast Lyapunov indicator and periodic orbits for symplectic mappings. Celestial Mechanics and Dynamical Astronomy, 81:129-147, 2001.

[51] E. Lega, M. Guzzo y Cl. Froeschlé. Detection of Arnold diffusion in Hamiltonian systems. Physica D: Nonlinear Phenomena, 182:179-187, 2003.

[52] Ch. Lhotka, Ch. Efthymiopoulos y R. Dvorak. Nekhoroshev instability at $l_{4}$ or $l_{5}$ in the elliptic restricted three-body problem - application to Trojan asteroids. Monthly Notices of the Royal Astronomical Society, 384:1165-1177, 2008.

[53] G. Lukes-Gerakopoulos, N. Voglis y Ch. Efthymiopoulos. The production of Tsallis entropy in the limit of weak chaos and a new indicator of chaoticity. Physica A, 387:1907-1925, 2008.

[54] A. Lyapunov. The general problem of the stability of motion. International Journal of Control, 55:531-773, 1992. Traducción inglesa de la versión en francés: Liapounoff A.: Problème général de la stabilité du mouvement. Anal. Fac. Sci. Toulouse. 9, 203-474 (1907). El orginal fue publicado en ruso por la Sociedad Matemática de Cracovia en 1892.

[55] N. Maffione. Comparación de indicadores de caos en sistemas hamiltonianos. Tesis doctoral en astronomía, FCAGLP, UNLP, Argentina, 2012.

[56] N. Maffione, L. Darriba, P. Cincotta y C. Giordano. A comparison of different indicators of chaos based on the deviation vectors. Application to symplectic mappings. Celestial Mechanics and Dynamical Astronomy, 111: 285-307, 2011.

[57] N. Maffione, C. Giordano y P. Cincotta. Testing a fast dynamical indicator: The MEGNO. International Journal of Nonlinear Mechanics, 46:23-34, 2011.

[58] N. Maffione, L. Darriba, P. Cincotta y C. Giordano. Chaos detection tools: applications to a self-consistent triaxial model. Monthly Notices of the Royal Astronomical Society, 429:2700-2717, 2013.

[59] J. Meiss. Symplectic maps, variational principles, and transport. Reviews in Modern Physics, 64:795-848, 1992.

[6o] M. Mestre. Difusión caótica en sistemas hamiltonianos casi-integrables. Tesis doctoral en astronomía, UNLP, Argentina, 2012.

[61] M. Mestre, P. Cincotta y C. Giordano. Analytical relation between two chaos indicators: FLI and MEGNO. Monthly Notices of the Royal Astronomical Society: Letters, 414, Issue 1:L100-L103, 2011. 
[62] A. Morbidelli. Modern celestial mechanics - Aspects of Solar System dynamics. Berlin Heidelberg: Taylor \& Francis/Cambridge Scientific Publishers, 2006.

[63] J.C. Muzzio, D. Carpintero y F. Wachlin. Spatial structure of regular and chaotic orbits in a sef-consistent triaxial stellar system. Celestial Mechanics and Dynamical Astronomy, 91, Issue 1-2:173-190, 2005.

[64] V. Oseledec. A multiplicative ergodic theorem. Lyapunov characteristic numbers for dynamical systems. Moscow Mathematical Society, 19:197-231, 1968.

[65] Y. Papaphilippou y J. Laskar. Frequency map analysis and global dynamics in a galactic potential of two degrees of freedom. Astronomy $\mathcal{E}$ Astrophysics, 307:427-449, 1996.

[66] Y. Papaphilippou y J. Laskar. Global dynamics of triaxial galactic models through frequency map analysis. Astronomy \& Astrophysics, 329:451-481, 1999 .

[67] Y. Petalas, C. Antonopoulos, T. Bountis y M. Vrahatis. Detecting resonances in conservative maps using evolutionary algorithms. Physics Letters $A$, 373:334-341, 2009.

[68] H. Poincaré. Les Méthodes nouvelles de la mécanique céleste. Gauthier-Villars et fils, París, Francia, 1892.

[69] H. Poincaré. La invención matemática. Ciencia y método, 1908.

[70] W. Press, S. Teukolsky, W. Vetterling y Flannery B. Numerical recipes in Fortran 77: the art of scientific computing. Vol. 1. Cambridge University Press, Cambridge, Reino Unido, 1992.

[71] P. Prince y J. Dormand. High order embedded Runge-Kutta formulae. Journal of Computational and Applied Mathematics, 7:67-75, 1981.

[72] S. Neil Rasband. The Poincaré map. Wiley, Nueva York, EE.UU., 1990.

[73] Z. Sándor, B. Érdi y Ch. Efthymiopoulos. The phase space structure around $l_{4}$ in the restricted three-body problem. Celestial Mechanics and Dynamical Astronomy, 78:113-123, 2000.

[74] Z. Sándor, B. Érdi, A. Széll y B. Funk. The relative Lyapunov indicator: An efficient method of chaos detection. Celestial Mechanics and Dynamical Astronomy, 90:127-138, 2004.

[75] G. Servizi, G. Turchetti, G. Benettin y A. Giorgilli. Resonances and asymptotic behavior of Birkhoff series. Physics Letters A, 95:11-14, 1983. 
[76] I. Shevchenko. Dynamical chaos in the Solar System. En: P. Cincotta, C. Giordano \& C. Efthymiopoulos eds.: 3rd La Plata International School on Astronomy and Geophysics "Chaos, Diffusion and Non-integrability in Hamiltonian Systems-Application to Astronomy.", 1:217-246, 2012.

[77] M. Sidlichovský y D. Nesvorný. Frequency modified Fourier transform and its applications to asteroids. Celestial Mechanics and Dynamical Astronomy, 65:137-148, 1997.

[78] Ch. Skokos. Alignment indices: a new, simple method for determining the ordered or chaotic nature of orbits. Journal of Physics A: Mathematical and General, 34:10029-10043, 2001.

[79] Ch. Skokos. The Lyapunov characteristic exponents and their computation. Lecture Notes in Physics, 790:63-135, 2010.

[8o] Ch. Skokos, G. Contopoulos y C. Polymilis. Structures in the phase space of a four dimensional symplectic map. Celestial Mechanics and Dynamical Astronomy, 65:223-251, 1997.

[81] Ch. Skokos, T. Bountis y Ch. Antonopoulos. Geometrical properties of local dynamics in Hamiltonian systems: The Generalized Alignment Index (GALI) method. Physica D: Nonlinear Phenomena, 231:30-54, 2007.

[82] Ch. Skokos, T. Bountis y Ch. Antonopoulos. Detecting chaos, determining the dimensions of tori and predicting slow diffusion in Fermi-Pasta-Ulam lattices by the Generalized Alignment Index method. The European Physical Journal Special Topics, 165:5-14, 2008.

[83] W. Tittemore y J. Wisdom. Tidal evolution of the Uranian satellites: I. Passage of Ariel and Umbriel through the 5:3 mean-motion commensurability. Icarus, 74:172-230, 1988.

[84] W. Tittemore y J. Wisdom. Tidal evolution of the Uranian satellites: II. An explanation of the anomalously high orbital inclination of Miranda. Icarus, 78:63-89, 1989 .

[85] N. Todorović, E. Lega y Cl. Froeschlé. Local and global diffusion in the Arnold web of a priori unstable systems. Celestial Mechanics and Dynamical Astronomy, 102:13-27, 2008.

[86] N. Voglis y G. Contopoulos. Invariant spectra of orbits in dynamical systems. Journal of Physics A: Mathematical and General, 27:4890-4909, 1994.

[87] N. Voglis, G. Contopoulos y Ch. Efthymiopoulos. Detection of ordered and chaotic motion using the dynamical spectra. Celestial Mechanics and Dynamical Astronomy, 73:211-220, 1999. 
[88] J. Wisdom, S. Peale y F. Mignard. The chaotic rotation of Hyperion. Icarus, 58:137-152, 1984 . 


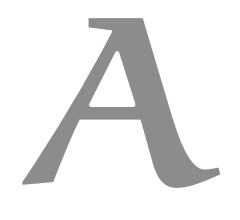

COMPOSICIÓN ENTRE SERIES DE FOURIER

Veremos a continuación un algoritmo general para realizar la composición entre funciones, las cuales todas pueden expresarse como desarrollos en series de Fourier, donde sus variables son ciertas acciones $\left(\mathrm{I}_{1}, \mathrm{I}_{2}\right)$ y sus ángulos conjugados serán $\left(\varphi_{1}, \varphi_{2}\right)$. Este algoritmo es el utilizado en la implementación de la rutina compose_functions (ver Apéndice C.5).

Consideremos una serie de Fourier de orden $j$ de la forma

$$
\sum_{\substack{s_{1}, s_{2}=0 \\ s_{1}+s_{2} \leqslant j}} \sum_{k_{1}, k_{2}=-\infty}^{\infty} e_{s_{1}, s_{2}, k_{1}, k_{2}} I_{1}^{s_{1}} I_{2}^{s_{2}} e^{i\left(k_{1} \varphi_{1}+k_{2} \varphi_{2}\right)},
$$

donde $\mathcal{C}_{s_{1}, s_{2}, k_{1}, k_{2}} \in \mathbb{C}$ es una constante, $j \in \mathbb{N}, s_{1}, s_{2} \in \mathbb{N}$ y $k_{1}, k_{2} \in \mathbb{Z}$.

Recordemos que, como se muestra en la Ec. (A.I), la dependencia de la serie de Fourier con las acciones es a través de potencias de éstas, es decir, que las acciones solamente aparecen en forma de monomios multiplicando a las expresiones exponenciales. Estas exponenciales, por su parte, son las que contienen la dependencia funcional con los ángulos, a través de una combinación lineal de ellas.

Veremos a continuación cómo realizar la composición $f\left(\mathbf{g}\left(\mathrm{I}_{1}, \mathrm{I}_{2}, \varphi_{1}, \varphi_{2}\right)\right)$ entre una función $\mathrm{f} y$ una función vectorial $\mathbf{g}=\left(\mathrm{g}_{\mathrm{I}_{1}}, \mathrm{~g}_{\mathrm{I}_{2}}, \mathrm{~g}_{\varphi_{1}}, \mathrm{~g}_{\varphi_{2}}\right)$, todas dependientes del conjunto de variables $\left(\mathrm{I}_{1}, \mathrm{I}_{2}, \varphi_{1}, \varphi_{2}\right)$ y expresadas como la Ec. (A.I).

Dado que la función $f$ se expresa como una serie de Fourier, para realizar la composición, tomaremos uno a uno cada término de $f$ y calcularemos la composición con respecto a las cuatro funciones g. Luego, el resultado se calcula como la suma de los términos luego de aplicar la composición.

Tomemos un dado término de nuestra función $f$ (lo llamaremos $f_{l}$ ) que será de la forma

$$
f_{l}=\mathcal{C}_{s_{1}, s_{2}, k_{1}, k_{2}} I_{1}^{s_{1}} I_{2}^{s_{2}} e^{i\left(k_{1} \varphi_{1}+k_{2} \varphi_{2}\right)}
$$


Vemos que en los términos considerados no tenemos en cuenta el parámetro de contabilidad $\lambda$. Esto es porque este parámetro solamente nos sirve para organizar los términos de acuerdo a su tamaño relativo, pero no influye en nuestros cálculos (recordemos que su valor numérico es $\lambda=1$ ).

Una vez que tenemos seleccionado dicho término, lo podemos descomponer como un producto de cuatro factores: dos factores correspondientes a las acciones $\mathrm{I}_{1}^{\mathrm{S}_{1}}$ e $\mathrm{I}_{2}^{\mathrm{S}_{2}}$, y los otros dos correspondientes a los ángulos, considerando a la exponencial como producto de dos exponenciales $e^{i k_{1} \varphi_{1}}$ y $e^{i k_{2} \varphi_{2}}$.

Consideremos uno de los factores dependiente de las acciones, por ejemplo $\mathrm{I}_{1}^{s_{1}}$ (para el factor $\mathrm{I}_{2}^{\mathrm{s}_{2}}$ el procedimiento es análogo). A partir de las reglas de composición, la acción $\mathrm{I}_{1}$ debe ser reemplazada por $\mathrm{g}_{\mathrm{I}_{1}}$, obteniendo

$$
I_{1}^{s_{1}}=g_{I_{1}}^{s_{1}}=\left(\sum_{j=0}^{N} \sum_{s_{1}^{\prime}+s_{2}^{\prime}=j} \sum_{k_{1}^{\prime}, k_{2}^{\prime}=-j}^{j} \mathcal{C}_{1, s_{1}^{\prime}, s_{2}^{\prime}, k_{1}^{\prime}, k_{2}^{\prime}} I_{1}^{s_{1}^{\prime}} I_{2}^{s_{2}^{\prime}} e^{i\left(k_{1}^{\prime} \varphi_{1}+k_{2}^{\prime} \varphi_{2}\right)}\right)^{s_{1}},
$$

donde, para poder realizar la implementación numérica, hemos truncado la serie de $\mathrm{g}_{\mathrm{I}_{1}}$ en un orden N. Luego la Ec. (A.3) es la expresión de la potencia de un polinomio en $\mathrm{I}_{1}$ e $\mathrm{I}_{2}$. El algoritmo que se empleó para su resolución es muy sencillo. Para calcular esta potencia se calculó el producto del polinomio que constituye $g_{I_{1}}$ por sí mismo, $s_{1}$ veces dentro de un bucle, utilizando la rutina product (ver Apéndice C.4).

Para evitar realizar operaciones innecesarias, luego de realizar una iteración (es decir, un producto $\mathrm{g}_{\mathrm{I}_{1}} \times \mathrm{g}_{\mathrm{I}_{1}}$ ), descartamos todos los términos que resulten de un orden mayor que el orden de precisión $\mathrm{N}$ con el que estamos calculando la composición. Luego, en la iteración siguiente, la cantidad de términos se ha reducido considerablemente, ya que de lo contrario habría términos de hasta orden $\mathrm{N}^{2}$ (por ser el producto de dos polinomios de grado $\mathrm{N}$ ). Este proceso se repite hasta que se hayan realizado los $s_{1}$ productos.

Veamos ahora cómo realizar la composición en los términos exponenciales. Aquí el cálculo de la composición es más complejo que en la situacion anterior. Consideremos el factor $e^{i k_{1} \varphi_{1}}$ (el caso para $e^{i k_{2} \varphi_{2}}$ es análogo). Para componer debemos reemplazar $\varphi_{1}$ por la función $g_{\varphi_{1}}$ en el exponente,

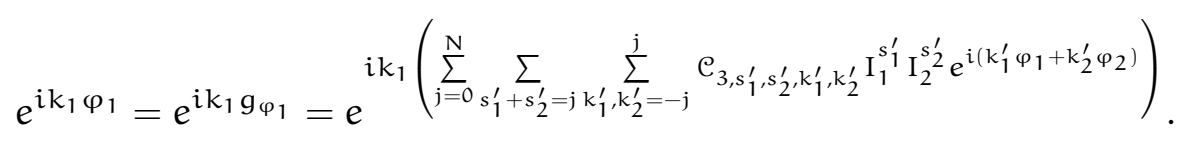


Para resolver esta composición debemos recordar que una función exponencial puede escribirse como la suma de los términos de su desarrollo de Taylor

$$
e^{x}=1+x+\frac{x^{2}}{2 !}+\frac{x^{3}}{3 !}+\cdots=\sum_{n=0}^{\infty} \frac{x^{n}}{n !}
$$

para una variable $x \in \mathbb{C}$. Si reemplazamos en la Ec. (A.5) la variable $x$ por $i k_{1} g_{\varphi_{1}}$, podremos obtener el desarrollo de Taylor de la Ec. (A.4)

$$
e^{i k_{1} g_{\varphi_{1}}}=1+i k_{1} g_{\varphi_{1}}+\frac{\left(i k_{1} g_{\varphi_{1}}\right)^{2}}{2 !}+\frac{\left(i k_{1} g_{\varphi_{1}}\right)^{3}}{3 !}+\cdots=\sum_{n=0}^{\infty} \frac{\left(i k_{1}\right)^{n}}{n !} g_{\varphi_{1}}^{n} \text {. (A.6) }
$$

Dentro de la sumatoria vemos que, a excepción del factor $\frac{\left(i k_{1}\right)^{n}}{n !}$, lo que nos queda es una potencia entera de la función $g_{\varphi_{1}}$. Esta función, al igual que $g_{I_{1}}$, es un desarrollo en series de Fourier, el cual lo truncamos a un dado orden N. Finalmente, la suma finita resultante la tratamos como hicimos más arriba para el caso de las potencias de las acciones, realizando una sucesión de productos de $g_{\varphi_{1}}$ con sí mismo y descartando, iteración a iteración, los términos de orden mayor que $\mathrm{N}$.

Una vez realizadas las 4 composiciones, solamente queda multiplicarlas entre sí para reconstruir el término que se estaba componiendo.

Recordemos que todo este procedimiento lo hicimos para un dado término de $f, f_{j}$. Este procedimiento debe repetirse para todos los otros términos de esta función. 



\section{FUNCIÓN DE INDEXACIÓN}

Recordemos, como ya hemos mencionado, que cada término del desarrollo de Fourier de los mapas $\mathcal{F}$ y $\mathcal{U}$, como también de la transformación $\Phi$, dependen de 5 índices enteros: $s_{1}$ y $s_{2}$ para las potencias de las acciones $\left(J_{i}, \Lambda_{i}\right), k_{1}$ y k para construir las combinaciones lineales de los ángulos $\left(x_{i}, \theta_{i}\right)$ y finalmente $r$ que es la potencia del parámetro de contabilidad $\lambda$ (Sección 5.I).

Es posible implementar, para cada una de estas funciones, el almacenamiento de dichos coeficientes utilizando un arreglo pentadimensional, donde cada celda contendrá el coeficiente correspondiente al término del desarrollo definido por estos índices. La principal desventaja de esta implementación es que, si realizamos un desarrollo de alto orden en $r$, la cantidad de términos que resultaría de la combinación de estos índices es muy alta, conllevando a que el arreglo resultante sea muy grande.

Presumiblemente, debido a que los índices siguen ciertas reglas, muchas de sus posibles combinaciones no serán utilizadas. Para comprender esto consideremos, por simplicidad, solamente los índices $s_{1}$ y $s_{2}$, que deben estar en el rango $0 \leqslant s_{i} \leqslant r$, con $i=1,2$ para un valor de $r$ (por el momento, para facilitar la explicación, prescindiremos de los índices $k_{1}$ y $k_{2}$ ). Si existe una restricción en los valores que pueden adoptar estos índices, por ejemplo $s_{1}+s_{2} \leqslant r$, la cantidad de posibles valores será $(r+1)(r+2) / 2$. Luego, si consideramos $r=15$, la cantidad de celdas necesarias para almacenar los términos válidos desde $r=0$ hasta $r=15$ serán 816. Sin embargo, el programa debe reservar el espacio en memoria para todo el arreglo completo, sin importar qué porcentajes de sus celdas se utilicen. En este ejemplo la cantidad de celdas será $(r+1) \times(r+1) \times(r+1)$, que resulta en un total de 4096 celdas. Podemos ver entonces que el programa solamente necesitaría aproximadamente $20 \%$ del espacio total reservado para el arreglo.

Este inconveniente es posible sortearlo mediante la implementación de un arreglo unidimensional que reemplace al arreglo pentadimensional que mencionamos en los párrafos anteriores. Dicho arreglo es generado a partir de los 5 índices involucrados, combinados de manera tal que cada quinteto de índices se corresponde con un único índice unidimensional $i$, y viceversa. La ventaja 
que presenta la implementación de este arreglo es que el algoritmo de generación de $i$ se construirá a partir de las reglas que restringen los posibles valores de los índices, de manera que solamente aparezcan aquéllos que son necesarios para el desarrollo. Veamos las reglas que le darán forma a este algoritmo.

Si bien todos los índices son enteros, $s_{1}, s_{2}$ y $r$ son en realidad enteros positivos. Llamemos $p=s_{1}+s_{2}$. Para cada desarrollo $\left(F_{i}, \Phi_{i}\right.$ y $U_{i}$, con $\left.i=1, \ldots, 4\right)$ tendremos una regla ligeramente distinta.

A continuación se describirá en detalle el algoritmo para la construcción del índice $i$ para el CRSSM. A partir de las expresiones del mapa $\mathcal{F}$ (Ec. (4.17)) es posible determinar cómo será la forma de los términos del desarrollo. Para las ecuaciones $F_{y_{i}}$, con $i=1,2$, tendremos que $s_{1}+s_{2}=1$, con $k_{1}=k_{2}=0$ y también $r=0$. Para los términos dependientes de los ángulos tendremos $\mp k_{1}= \pm k_{2}=r$, y también $k_{i}= \pm r, k_{j}=0$, con $i \neq j$. Si consideramos las funciones $F_{x_{i}}$ las expresiones son análogas, sólo que $r$ se ve incrementado en 1 en todas las expresiones.

En cuanto a las ecuaciones de las transformaciones $\Phi_{j}$ con $j=J_{1}, J_{2}, x_{1}, x_{2}$ las reglas son ligeramente más complejas, resultando

$$
\Phi_{J_{i}}(i=1,2) \rightarrow\left\{\begin{array} { l } 
{ 0 \leqslant p \leqslant r } \\
{ 0 \leqslant p + | k _ { i } | \leqslant r } \\
{ 0 \leqslant | k _ { i } | \leqslant r }
\end{array} \quad \Phi _ { x _ { i } } ( i = 1 , 2 ) \rightarrow \left\{\begin{array}{l}
0 \leqslant p \leqslant r-1 \\
0 \leqslant p+\left|k_{i}\right| \leqslant r-1 \\
0 \leqslant\left|k_{i}\right| \leqslant r-1 .
\end{array}\right.\right.
$$

Por último, en las ecuaciones de la forma normal $u_{j}$ con $j=\Lambda_{1}, \Lambda_{2}, \theta_{1}, \theta_{2}$, no aparecen todos los órdenes de $r$, sino que comienzan a partir de $r_{0}=4$ para $U_{\Lambda_{i}}$ y $r_{0}=5$ para $U_{\theta_{i}}$, con $i=1$, 2. Luego las reglas son análogas a las anteriores

$$
\mathrm{u}_{j}\left(\mathrm{j}=\Lambda_{1}, \Lambda_{2}, \theta_{1}, \theta_{2}\right) \rightarrow\left\{\begin{array}{l}
0 \leqslant p \leqslant r-r_{0}+1 \\
0 \leqslant p+\left|k_{i}\right| \leqslant r-r_{0}+1 \\
0 \leqslant\left|k_{i}\right| \leqslant r-r_{0}+1
\end{array}\right.
$$

Por una cuestión de simplicidad en el código, hemos adoptado una regla más general, similar a las anteriores. Esta consiste en considerar simplemente

$$
\left\{\begin{array}{l}
0 \leqslant p \leqslant r \\
0 \leqslant\left|k_{i}\right| \leqslant r .
\end{array}\right.
$$

El algoritmo utilizado para generar los valores es el siguiente: 
Primero se comprueba si los valores del quinteto $\left(s_{1}, s_{2}, k_{1}, k_{2}, r\right)$ están dentro de los rangos establecidos en el Apéndice B. Una vez que está comprobado esto se procede a la construcción del índice. Dicha construcción consta de tres partes.

En la primera parte se cuenta, mediante la función cell_starting_lambda(r), cuántas celdas han sido ocupadas hasta el orden $r-1$ (que obviamente contempla todas las posibles combinaciones de $s_{1}, s_{2}, k_{1} y k_{2}$ ).

En cuanto a la segunda parte (almacenada en la variable B) dado que $s_{1}+$ $s_{2} \leqslant r$, se define el valor auxiliar $p=s_{1}+s_{2}$. Luego luego se cuentan todas las celdas ocupadas hasta $p-1$ más las correspondientes a $p$ hasta alcanzar el valor de $s_{1}$, mediante la expresión

$$
B=\left(\frac{p(p+1)}{2}+s_{1}\right)(2 r+1)(2 r+1),
$$

donde $p(p+1) / 2+s_{1}$ cuenta la cantidad de combinaciones de $s_{1}$ y $s_{2}$ para valores fijos de $k_{1} y k_{2}$. El doble factor $2 r+1$ es para considerar, para cada par de valores $\left(s_{1}, s_{2}\right)$, todas las combinaciones de $k_{1}$ y $k_{2}$. Como éstos pueden variar desde $-r$ hasta $r$, tenemos $2 r+1$ posibles valores para cada uno.

Finalmente, en la tercera parte de esta construcción, calculada mediante la expresión

$$
C=\left(2\left|k_{1}\right|-l_{1}\right)(2 r+1)+\left(2\left|k_{2}\right|-l_{2}\right)
$$

se cuenta, en el primer término, todas las combinaciones de $k_{1}(2 r+1) y$, en el segundo término, la posición relativa del índice $k_{2}$, para $s_{1}, s_{2}$ y $k_{1}$ fijos. Dado que $k_{i}$, con $i=1,2$ pueden tomar valores tanto positivos como negativos, la forma de ordenarlos fue considerar el valor absoluto de éstos, y luego colocar, en una dada celda, el término correspondiente al valor positivo de $k_{i}$ y en la celda siguiente el correspondiente al valor negativo (como se muestra en la Tabla de la Sección C.4), mediante la introducción de dos variables $l_{i}$. Si el valor de $k_{i}$, con $i=1,2$ es positivo, $l_{i}$ valdrá 1 , y 0 en caso contrario.

Para el caso particular de $r=0$ se produce una excepción a la regla $s_{1}+$ $s_{2} \leqslant r$, ya que aparecen en las expresiones para las acciones (Ec. (4.17)) los términos $y_{i}$, con $i=1,2$. Cuando se construye la función de indexación se debe contemplar esta configuración. En vez que considerar que los $s_{i}$ pueden solamente adoptar el valor 1 , se contempla la posibilidad de que estas potencias puedan ir a valores más altos. Para ello se define el parámetro MAXINDR0, se reservan las primeras 2MAXINDRO celdas para almacenar MAXINDRO potencias de $s_{2}$ con $s_{1}=0$, y una cantidad similar para las potencias de $s_{1}$ con $s_{2}=0$. 


\begin{tabular}{|rrrrr|r|}
\hline$s_{1}$ & $s_{2}$ & $k_{1}$ & $k_{2}$ & $r$ & $i$ \\
\hline 0 & 0 & 0 & 0 & 0 & 0 \\
1 & 0 & 0 & 0 & 0 & 1 \\
0 & 1 & 0 & 0 & 0 & 2 \\
0 & 0 & 0 & 0 & 1 & 3 \\
0 & 0 & 0 & 1 & 1 & 4 \\
0 & 0 & 0 & -1 & 1 & 5 \\
0 & 0 & 1 & 0 & 1 & 6 \\
0 & 0 & 1 & 1 & 1 & 7 \\
0 & 0 & 1 & -1 & 1 & 8 \\
0 & 0 & -1 & 0 & 1 & 9 \\
0 & 0 & -1 & 1 & 1 & 10 \\
0 & 0 & -1 & -1 & 1 & 11 \\
0 & 1 & 0 & 0 & 1 & 12 \\
0 & 1 & 0 & 1 & 1 & 13 \\
0 & 1 & 0 & -1 & 1 & 14 \\
\hline
\end{tabular}

\begin{tabular}{|rrrrr|r|}
\hline$s_{1}$ & $s_{2}$ & $k_{1}$ & $k_{2}$ & $r$ & $i$ \\
\hline 0 & 1 & 1 & 0 & 1 & 15 \\
0 & 1 & 1 & 1 & 1 & 16 \\
0 & 1 & 1 & -1 & 1 & 17 \\
0 & 1 & -1 & 0 & 1 & 18 \\
0 & 1 & -1 & 1 & 1 & 19 \\
0 & 1 & -1 & -1 & 1 & 20 \\
1 & 0 & 0 & 0 & 1 & 21 \\
1 & 0 & 0 & 1 & 1 & 22 \\
1 & 0 & 0 & -1 & 1 & 23 \\
1 & 0 & 1 & 0 & 1 & 24 \\
1 & 0 & 1 & 1 & 1 & 25 \\
1 & 0 & 1 & -1 & 1 & 26 \\
1 & 0 & -1 & 0 & 1 & 27 \\
1 & 0 & -1 & 1 & 1 & 28 \\
1 & 0 & -1 & -1 & 1 & 29 \\
\hline
\end{tabular}

Tabla B.1: Ordenamiento de los índices para $r=0$ y $r=1$ aplicando el algoritmo implementado en la función de indexación.

Finalmente, el valor de salida de esta función será simplemente

$$
\text { index_function }=\text { cell_starting_lambda }(r)+B+C \text {. }
$$

En la Tabla B.I mostramos, a modo de ejemplo, el ordenamiento de los índices para $r=0$ y $r=1$. 
FORMAS NORMALES: EL CÓDIGO

Como se ha visto en la segunda parte de este trabajo, particularmente en el Capítulo 4, la transformación a las formas normales se realiza mediante la implementación de un algoritmo no trivial. Es por esta razón que se creó un paquete de programas que implementa dicho algoritmo.

El objetivo de este paquete es, en términos generales, calcular la forma normal de un mapa simpléctico 4D en particular, el CRSSM (ver Sección 4.2 para más detalles). Para poder implementar la transformación de forma más eficiente y modular, dicho paquete está dividido en tres grandes programas cuya funciones están bien definidas:

1. Construcción de los desarrollos de las series de Fourier de las ecuaciones del mapa CRSSM (build_mapping.f90).

2. Cálculo de la transformación a la forma normal $(\Phi)$, el desarrollo en series de Fourier de las ecuaciones del mapa transformado (U) y, más importante aún para nuestros estudios, la transformación inversa $(\Psi)$ (main.f90).

3. Aplicación de la transformación inversa a la serie de tiempo en las variables originales para el posterior cálculo de la desviación cuadrática media en las nuevas variables canónicas (mqd.f90).

Estos tres programas requieren de diversas rutinas de cálculo, como la composición entre series de Fourier, o la comprobación de la simplecticidad de la transformación. Es por ello que están acompañados de diversos archivos que contienen dichas rutinas, separadas de acuerdo a la afinidad en sus funciones:

1. public_values. $f 90$

2. routines. $f 90$

3. arithmetic_routines. $f 90$

4. compose_function. $f 90$

5. inverse_transformation. $f 90$ 
6. precision. $f 90$

las cuales serán descriptas en detalle a continuación.

\section{C.I CONSIDERACIONES GENERALES}

Antes de describir uno a uno cada archivo que compone el paquete que calcula la forma normal, dediquemos esta primera parte a definir algunos conceptos básicos que fueron utilizados en el desarrollo del código, y son necesarios para comprender algunos de los algoritmos implementados.

\section{C.1.1 Series y arreglos}

Como bien sabemos, para el cálculo de la forma normal hemos considerado que, tanto las ecuaciones del mapa en las variables canónicas originales $F_{i}$, de la transformación $\Phi_{i}$ y del mapa en las variables nuevas $U_{i}$, están formadas por una serie de Fourier (truncadas a orden $\mathrm{N}$ ) donde las acciones solamente forman parte de los coeficientes y aparecen como una potencia entera $\left(y_{1}^{s_{1}}\right.$ y $\left.y_{2}^{s_{2}}\right)$, mientras que los ángulos solamente aparecen como argumento de la función exponencial, mediante una combinación lineal del tipo $k_{1} x_{1}+k_{2} x_{2}$.

Para facilitar la manipulación en el código y poder hacer operaciones de forma algebraica sin perder precisión, hemos almacenado cada una de estas expresiones en un arreglo unidimensional (ver Apéndice B para comprender cómo almacenar en un arreglo unidimensional una serie que depende de 5 índices), donde el índice del arreglo nos dice qué término de la serie es el que estamos considerando (correspondiente al quinteto de índices $s_{1}, s_{2}, k_{1}, k_{2}$ y r), y el contenido de dicha celda es el valor del coeficiente que multiplica al factor $y_{1}^{s_{1}} y_{2}^{s_{2}} e^{i\left(k_{1} x_{1}+k_{2} x_{2}\right)} \lambda^{r}$

Mediante esta consideración podremos realizar todas las operaciones aritméticas que veremos más adelante de forma rápida y ágil, sin perder precisión en el proceso.

Las secciones siguientes serán dedicadas a definir y describir las rutinas auxiliares necesarias para llevar a cabo la transformación.

\section{C.2 PUBLIC_VALUES.F90}

Este archivo es una compilación de declaraciones y definiciones de parámetros. Algunos de estos parámetros corresponden al mapa en cuestión, mientras que otros son parámetros propios del desarrollo de las formas normales.

A continuación detallamos las variables declaradas en este módulo correspondientes a las propiedades de los desarrollos de Fourier. 
- cells_used_lambda: Arreglo entero que almacena, en la celda $i$, la cantidad de celdas que ocupa el desarrollo del mapa $\mathcal{F}$ hasta el orden $i-$ ésimo.

- cells_starting_lambda: Arreglo entero que indica, en la celda $i$, en qué celda comienzan a almacenarse los términos de orden $\lambda^{i}$.

- cells_ending_lambda: Arreglo entero que indica, en la celda $i$, en qué celda se encuentra el último término correspondiente al orden $\lambda^{i}$.

- s1ind, s2ind, k1ind, k2ind, rind: Estos cinco arreglos también son enteros y guardan, en la celda $i$, el valor correspondiente de cada índice $s_{1}, s_{2}, k_{1}, k_{2}$ y $r$, luego de generar la función de indexación y la correspondiente función de indexación inversa. Por ejemplo, el índice 774 corresponde al término $\lambda^{3} y_{1}^{2} y_{2} e^{i\left(-2 x_{2}\right)}$. Luego, las celdas correspondientes a estos arreglos contendrán los valores: $\operatorname{sind}(774)=2$, s2ind $(774)=1$, $\mathrm{k} 1$ ind $(774)=0, \mathrm{k} 2$ ind $(774)=-2$, rind $(774)=3$.

Existen otros parámetros que han sido implementados en el código, como por ejemplo, maxlambda, que contiene el orden máximo posible para la potencia de $\lambda$, para poder tener un corte en el desarrollo, o ncoeffmax, que limita el número máximo de términos a calcular en el desarrollo de Fourier.

Una segunda serie de parámetros define las propiedades del mapa $F$ que vamos a transformar.

Se definen cuatro parámetros, eps1, eps2, gamp, gamm que contienen los valores de $\varepsilon_{1}, \varepsilon_{2}, \gamma_{+} \mathrm{y} \gamma_{-}$, respectivamente, correspondientes al mapa CRSSM.

Si bien en este trabajo se considera una simetría en los parámetros perturbativos del mapa, donde $\varepsilon_{1}=\varepsilon_{2}=\varepsilon$ y $\gamma_{+}=\gamma_{-}=\gamma$, el código contempla la posibilidad de que éstos no sean iguales y se puedan considerar de forma independiente.

Recordemos que en este trabajo se consideró una variante normalizada del mapa. Por lo tanto, el parámetro $\varepsilon_{i}(i=1,2)$ es reemplazado por $\varepsilon_{i}^{2}$ y, $\gamma_{+} o \gamma_{-}$ son reemplazados por $\gamma_{ \pm} \varepsilon_{i}$. Luego, se introducen los parámetros eps12, eps22, gamp1, gamp2, gamm1, gamm2 que almacenan $\varepsilon_{1}^{2}, \varepsilon_{2}^{2}, \gamma_{+} \varepsilon_{1}, \gamma_{+} \varepsilon_{2}, \gamma_{-} \varepsilon_{1}$ y $\gamma_{-} \varepsilon_{2}$, respectivamente.

También se definen dos arreglos de dimensión 3, mu y phi, que contienen los valores de $\mu_{i}$ y $\varphi_{i}, i=1,2,3$. Por último, como parámetros correspondientes al mapa se definen dos, omega10 y omega20, que contienen los valores centrales $\omega_{1}^{*}$ y $\omega_{2}^{*}$, alrededor de los cuales se lleva a cabo la transformación de las formas normales.

Finalmente, también se definen en este archivo ciertos valores generales de cantidades conocidas, como el valor de $\pi$, o un valor umbral considerado como "cero numérico"de $10^{-16}$. 
Este archivo contiene un conjunto de rutinas que realizan las operaciones que forman parte del cálculo de la forma normal.

- index_function: Esta función calcula, a partir de los 5 índices $s_{1}, s_{2}, k_{1}$, $k_{2}$ y $r$, un único índice, de manera de poder almacenar todos los términos de un desarrollo de Fourier en un arreglo unidimensional. Esto lo hace de acuerdo al algoritmo de indexación detallado en el Apéndice B.

- build_inverse_index_function: Esta rutina realiza el proceso inverso al de la rutina index_function. Genera 5 arreglos unidimensionales (uno para cada índice) donde, para el índice $i$-ésimo almacena el valor correspondiente de cada índice $s_{1}, s_{2}, k_{1}, k_{2} \mathrm{y} r$. Esta rutina es la que construye los arreglos de índices s1ind, s2ind, k1ind, k2ind y rind que se definen en el archivo public_values.f90 (Sección C.2).

- check_index_range: Cuando el programa llama a la función de índices, ésta llama primero a esta rutina, la cual comprueba si los índices que son argumentos de la función de índices están en el rango permitido (de acuerdo a las restricciones que presentan los índices) o no.

- cells_counting_lambda: Calcula cuántas celdas están siendo ocupadas por todos los términos hasta un cierto orden $k$ de $\lambda$ y lo almacena en el elemento $k$ de un arreglo unidimensional.

\section{C.4 ARITHMETIC_ROUTINES.F90}

Este archivo es una compilación de rutinas que resuelven operaciones aritméticas entre arreglos. Son particularmente útiles ya que una gran parte de los cálculos que debemos realizar son operaciones entre series de Fourier, las cuales son almacenadas en arreglos. Las rutinas que conforman este archivo son:

- product: Realiza el producto entre dos series de Fourier, calculando el producto de cada término de una serie con cada término de la otra serie.

- power: Calcula la potencia de una serie de Fourier elevada a un exponente pow $\in \mathbb{N}$, mediante la aplicación sucesiva de la rutina product para realizar el producto del arreglo (donde se encuentra almacenada la serie) por sí mismo pow -1 veces. 
- build_local_function: Ocurre frecuentemente que, en el desarrollo del método del cálculo de formas normales, solo algunas de las combinaciones de índices en la serie de Fourier contienen coeficientes no nulos. Por ejemplo, las ecuaciones del mapa en las variables canónicas originales (Ec. (4.17)), contienen solamente a las acciones mediante una dependencia lineal y en términos que no dependen de los ángulos, esto es $s_{i}=1, s_{j}=0$ con $i, j=1,2$ e $i \neq j, y k_{1}=k_{2}=0$. Por ende, todas aquellas celdas que representen términos cuyos índices no correspondan a este tipo de combinación, estarán vacías, ya que no forman parte de la expresión del mapa. Esto hace que el arreglo que se está utilizando presente un tamaño considerablemente mayor al que realmente es necesario.

La función de esta rutina es, a partir de un arreglo de entrada, generar dos nuevos arreglos de salida: uno con coeficientes complejos y el otro con coeficientes enteros. En el primero irán almacenados los coeficientes no nulos del arreglo de entrada y, en el segundo, irán los índices asociados a los coeficientes correspondientes. De esta manera se puede reducir en más de un orden de magnitud el tamaño del arreglo y, por consiguiente, el número de operaciones. Veamos un ejemplo de esto:

Supongamos que tenemos un arreglo donde los índices $s_{1}, s_{2}, k_{1}, k_{2}$ y $r$ cumplen lo siguiente:

$$
\begin{array}{r}
0 \leqslant s_{1}, s_{2} \leqslant 5 \\
-5 \leqslant k_{1}, k_{2} \leqslant 5 \\
0 \leqslant r \leqslant 5 .
\end{array}
$$

Con estos valores tendremos un total de $6 \times 6 \times 11 \times 11 \times 6=26136$ términos, y supongamos que solamente son no nulos aquéllos para los que se cumple alguna de las siguientes condiciones:

$$
\begin{array}{lr}
s_{1}=1, s_{2}=0 \text { con } k_{1}=k_{2}=0 y r=1 & (1 \text { término }) \\
s_{1}=0, s_{2}=1 \text { con } k_{1}=k_{2}=0 y r=1 & (1 \text { término }) \\
k_{1}=1, k_{2}=0, s_{1}=s_{2}=0, \text { con } r=1 & (1 \text { término }) \\
k_{1}=0, k_{2}=1, s_{1}=s_{2}=0, \text { con } r=1 & (1 \text { término }) \\
s_{1}=s_{2}=0, k_{1}= \pm k_{2}, y 1 \leqslant\left|k_{1}\right|,\left|k_{2}\right| \leqslant 5 & (20 \text { términos })
\end{array}
$$

que es, básicamente, la forma del desarrollo de Fourier de la Ec. (4.17) para $y_{1}^{\prime}$. Esta reconstrucción del arreglo reduce el número de términos a solamente 24. Supongamos ahora que debemos realizar el producto entre 
dos desarrollos de Fourier cuyos términos siguen las reglas mencionadas más arriba. Si consideramos el arreglo completo, el número de operaciones sería $26136^{2} \approx 7 \times 10^{8}$ operaciones mientras que, si utilizamos la optimización implementada en esta rutina, el número de operaciones es simplemente 576, es decir 6 órdenes de magnitud menor. Esto muestra la utilidad de la implementación de esta subrutina.

- derivative: Calcula la derivada de un desarrollo de Fourier con respecto a alguna de las 4 variables. Esto es muy sencillo de realizar ya que cualquiera de estas derivadas es o bien la derivada de una potencia (en el caso de las acciones) o bien la derivada de una exponencial (para el caso de los ángulos).

El algoritmo cuenta con un argumento llamado Aflag que nos indica si está presente en el desarrollo un término aditivo de la forma $x_{1}$ o $x_{2}$. Esto es debido a que, como se puede ver en la Ec. (4.17), los desarrollos de $x_{i}^{\prime}$ con $i=1,2$ presentan un término de la forma $x_{i}$, que no es posbile escribirlo en la forma de un término de una serie de Fourier, por lo que su derivada tiene que ser tratada aparte, presentando una resolución trivial.

- integrate: Esta subrutina, al contrario que la anterior, calcula la primitiva del desarrollo de Fourier, utilizando la operación inversa a la utilizada para derivar.

- addarrays: Realiza la suma (o resta) de dos desarrollos de Fourier y lo almacena en un tercer arreglo.

- poisson: Calcula los corchetes de Poisson entre dos desarrollos y lo almacena en un tercero. Esta operación la lleva a cabo mediante la combinación de las rutinas derivative, product y addarrays.

- check_symplectic: Comprueba si la transformación es simpléctica hasta el orden nord que se le pasa como argumento o no, auxiliándose en la anterior rutina para calcular los corchetes de Poisson.

\section{C.5 COMPOSE_FUNCTIONS.F90}

Este archivo consta de una única rutina, llamada compose que toma, como entrada, cinco funciones (arreglos), llámense $f, g_{y_{1}}, g_{y_{2}}, g_{x_{1}}$ y $g_{x_{2}}$. Esta rutina toma la función $\mathrm{f}$ y la compone con las otras cuatro, es decir

$$
f\left(y_{1}, y_{2}, x_{1}, x_{2}\right) \rightarrow(f \circ g)\left(y_{1}, y_{2}, x_{1}, x_{2}\right)=f\left(g_{y_{1}}, g_{y_{2}}, g_{x_{1}}, g_{x_{2}}\right) .
$$

Recordemos que, tanto la función $f$ como las funciones $g_{j}\left(j=y_{1}, y_{2}, x_{1}, x_{2}\right)$ son todas series de Fourier. Por ende, el algoritmo de composición tiene que 
ser capaz de componer expresiones de este tipo. En el Apéndice A se explica cómo se implementa la composición entre series de Fourier.

\section{C.6 INVERSE_TRANSFORMATION.F90}

Este archivo, al igual que compose_functions.f90, consta de una sola rutina, llamada inverse. Como el nombre del archivo sugiere, la función de esta rutina es calcular la transformación inversa a la forma normal. Está formada por cuatro bloques de código donde cada uno, a través de la utilización de la rutina compose, resuelve la Ec. (4.71) para cada uno de los cuatro $\Psi_{i}$, con $i=1, \ldots, 4$.

\section{C.7 BUILD_MAPPING.F 90}

Como hemos visto, todos los archivos mencionados hasta aquí son simplemente una compilación de rutinas. Este archivo es uno de los tres programas que constituyen el paquete que calcula la transformación a las formas normales, junto con main. $f 90$ y mqd. $f 90$.

Éste es el primer programa del paquete que debe ejecutarse. Su función consiste en calcular y construir el desarrollo de Fourier del mapa en el conjunto original de variables (en esta versión del programa es el CRSSM). Una vez calculado el desarrollo de las cuatro funciones $\left(F_{y_{1}}, F_{y_{2}}, F_{x_{1}} y F_{x_{2}}\right)$, las guarda en archivos separados, para luego ser utilizados por el programa main. $f 90$.

\section{C.8 MAIN.F90}

Como su nombre en inglés sugiere, este archivo es el programa principal para la transformación a formas normales. Describiremos someramente la estructura del mismo.

Primero llama a la función cells_counting_lambda que es necesaria para la construcción de la función de índices. Luego de esto invoca a la función build_inverse_index_function para construir los arreglos de índices s1ind, s2ind, k1ind, k2ind y rind. Seguido de esto, lee los coeficientes de las series $F_{i}$ que constituyen el mapa original, construidos por el programa build_mapping.

Una vez que el programa principal ha inicializado todos los arreglos y leído los coeficientes del mapa original, ingresa en un bucle sobre una variable Nord que contabiliza el orden del desarrollo que se está calculando, hasta un orden máximo Nmaxexp. Para cada orden, el programa realiza los siguientes pasos.

Primero realiza las composiciones $F_{i} \circ \boldsymbol{\Phi}$ y $\Phi_{i} \circ \mathbf{U}$ para construir la ecuación homológica (Sección 4.4.4). Una vez construida la ecuación homológica, inicia el algoritmo para su resolución. Esto se lleva a cabo analizando uno a uno 
los términos que forman parte de los arreglos $Q_{i}$ (Ec. (4.47)), para determinar cuáles de ellos pertenecen al nuevo mapa $U_{i}$ y cuáles a la transformación $\Phi_{i}$.

Una vez finalizada la resolución de la ecuación, el programa invoca ahora a la subrutina check_simplectic para comprobar si, hasta el orden actual, la transformación es simpléctica. En caso de no serlo, compensará los términos excedentes para que sí lo sea, como se ha explicado en la Sección 4.4.5.

Una vez que el programa construye las transformaciones $\Phi_{i}$ y las ecuaciones $U_{i}$ del nuevo mapa hasta el orden Nexpmax, sale del bucle. Luego, el programa construye las transformaciones inversas $\Psi_{i}$ mediante el llamado a la subrutina inverse.

Finalmente, se escriben los resultados de la transformación $\left(\Psi_{\Lambda_{1}}, \Psi_{\Lambda_{2}}, \Psi_{\theta_{1}}\right.$ y $\Psi_{\theta_{2}}$ ) en diferentes archivos, como también los coeficientes de $U_{i}$ y $\Phi_{i}$.

C.9 MQD.F90

Este es el último archivo que compone el paquete. Está formado por el programa principal y una función, llamada evaluate_function. El objetivo de esta última es calcular la evaluación de una función en un punto del espacio de fases. Primero toma como entrada una función (es decir, un arreglo) y los valores del punto $\left(y_{1}, y_{2}, x_{1}, x_{2}\right)$, ya sean éstos en las variables viejas o nuevas. Construye, término a término, el producto $y_{1}{ }^{s_{1}} y_{2}{ }^{s_{2}} e^{i\left(k_{1} x_{1}+k_{2} x_{2}\right)}$, y luego lo multiplica por su correspondiente coeficiente. Luego suma todos los términos calculados, obteniendo finalmente la evaluación de la función $f\left(y_{1}, y_{2}, x_{1}, x_{2}\right)$ para un punto $\left(y_{1}, y_{2}, x_{1}, x_{2}\right)$ dado.

Con respecto al bloque principal que contiene este archivo, su nombre proviene de su acrónimo en inglés mean quadratic deviation, es decir, desviación cuadrática media. Como lo dice su nombre, este programa calcula la d.c.m. El flujo del código es el siguiente.

Primero el programa lee, desde un archivo de entrada, la serie de tiempo de las coordenadas del espacio de fases en las variables originales $\left(J_{1}, J_{2}, \chi_{1}, \chi_{2}\right)$ con las cuales construirá la nueva serie de tiempo en las variables transformadas $\left(\Lambda_{1}, \Lambda_{2}, \theta_{1}, \theta_{2}\right)$. Luego de esto lee los coeficientes de la transformación inversa $\Psi$, que fue calculada con el programa main. $\mathbf{f} 90$.

Una vez leída la serie de tiempo y los coeficientes de la tranformación inversa, el programa construye la nueva serie de tiempo, a partir de la evaluación de las funciones $\Psi_{i}$ en los puntos $\left(J_{1}, J_{2}, x_{1}, x_{2}\right)$.

Con la nueva serie de tiempo construida, el programa calcula la d.c.m., en ambos conjuntos de variables, y tanto en la dirección de la resonancia $\left(\mathrm{I}_{\mathrm{f}}\right)$ como en la dirección perpendicular a ésta $\left(I_{R}\right)$, y lo guarda en un archivo de salida. 